JOURNAL OF THE

AMERICAN MATHEMATICAL SOCIETY

S 0894-0347(2014)00786-4

Article electronically published on March 5, 2014

\title{
EISENSTEIN CONGRUENCE ON UNITARY GROUPS AND IWASAWA MAIN CONJECTURES FOR CM FIELDS
}

\author{
MING-LUN HSIEH
}

\section{Contents}

Introduction

1. Notation and conventions

2. Shimura varieties for unitary groups

3. Modular forms on unitary groups

4. Hida theory for unitary groups

5. Ordinary $p$-adic Eisenstein series on $U(2,1)$

6. Constant terms of the $p$-adic Eisenstein series

7. Eisenstein ideal and $p$-adic $L$-functions $\quad 76$

8. Application to the main conjecture for CM fields 93

Acknowledgments 107

References 107

\section{INTRODUCTION}

The main conjecture for CM elliptic curves over totally real fields. Let $\mathcal{M}$ be an imaginary quadratic field and let $E$ be an elliptic curve over a totally real field $\mathcal{F}$ with complex multiplication by the ring of integers $\mathcal{O}_{\mathcal{M}}$ of $\mathcal{M}$. Let $p$ be an odd prime split in $\mathcal{M}$. Let $\mathcal{F}_{\infty}$ be the cyclotomic $\mathbf{Z}_{p}$-extension of $\mathcal{F}$ and let $\Lambda_{\mathcal{F}}:=\mathbf{Z}_{p} \llbracket \operatorname{Gal}\left(\mathcal{F}_{\infty} / \mathcal{F}\right) \rrbracket$ be a one-variable Iwasawa algebra. We study the cyclotomic main conjecture of Iwasawa theory for $E$ which relates the size of Selmer groups to special values of the $p$-adic $L$-function attached to $E$. Recall that the Selmer group $\operatorname{Sel}_{\mathcal{F}_{\infty}}(E)$ is defined by

$$
\operatorname{Sel}_{\mathcal{F}_{\infty}}(E)=\operatorname{ker}\left\{H^{1}\left(\mathcal{F}_{\infty}, E\left[p^{\infty}\right]\right) \rightarrow \prod_{v} H^{1}\left(\mathcal{F}_{\infty, v}, E\right)\right\},
$$

where $v$ runs over all places of $\mathcal{F}_{\infty}$. It is well known that $\operatorname{Sel}_{\mathcal{F}_{\infty}}(E)$ is a cofinitely generated $\Lambda_{\mathcal{F}}$-module. Denote by $\operatorname{char}_{\Lambda_{\mathcal{F}}} \operatorname{Sel}_{\mathcal{F}_{\infty}}(E)$ the characteristic power series of $\operatorname{Sel}_{\mathcal{F}_{\infty}}(E)$, which is an element in $\Lambda_{\mathcal{F}}$ unique up to a $\Lambda_{\mathcal{F}}$-unit. Let $\mathcal{W}_{p}$ be the $p$-adic completion of the ring of integers of the maximal unramified extension of $\mathbf{Z}_{p}$. On the other hand, the specialization of a suitable twist of a Katz $p$-adic $L$-function to the cyclotomic line yields a $p$-adic $L$-function $L_{p}\left(E / \mathcal{F}_{\infty}\right) \in \Lambda_{\mathcal{F}, \mathcal{W}_{p}}:=\Lambda_{\mathcal{F}} \otimes \mathbf{z}_{p} \mathcal{W}_{p}$, which roughly interpolates the algebraic part of central $L$-values $L\left(E_{/ \mathcal{F}} \otimes \nu, 1\right)$ twisted by finite order characters $\nu: \operatorname{Gal}\left(\mathcal{F}_{\infty} / \mathcal{F}\right) \rightarrow \mathbf{C}^{\times}$(see 88.6 .2 for the precise

Received by the editors June 18, 2012 and, in revised form, April 17, 2013.

2010 Mathematics Subject Classification. Primary 11R23 11F70, Secondary 11F33.

(C)2014 American Mathematical Society 
definition). The main conjecture of Mazur and Swinnerton-Dyer for $E$ predicts the following equality between ideals in $\Lambda_{\mathcal{F}, \mathcal{W}_{p}}$ :

Conjecture 1 (The main conjecture for CM elliptic curves).

$$
\left(\operatorname{char}_{\Lambda_{\mathcal{F}}} \operatorname{Sel}_{\mathcal{F}_{\infty}}(E)\right)=\left(L_{p}\left(E / \mathcal{F}_{\infty}\right)\right) .
$$

If $\mathcal{F}=\mathbf{Q}$ and $E$ has good ordinary reduction at $p$, the above conjecture is a theorem of K. Rubin Rub91, Thm. 12.3]. Using the main result in this article, we obtain one-sided divisibility in the main conjecture for a class of CM elliptic curves over totally real fields. To state our theorem, we need some notation. Let $\mathcal{K}=\mathcal{F} \mathcal{M}$ and let $D_{\mathcal{K} / \mathcal{F}}$ be the relative discriminant of $\mathcal{K} / \mathcal{F}$. If $L$ is a number field, let $h_{L}$ be the class number of $L$ and $D_{L}$ be the absolute discriminant of $L$. Let $h_{\mathcal{K}}^{-}=h_{\mathcal{K}} / h_{\mathcal{F}}$ be the relative class number of $\mathcal{K} / \mathcal{F}$. One of our main results is as follows.

Theorem 1. Suppose that $p \nmid 6 \cdot h_{\mathcal{K}}^{-} \cdot D_{\mathcal{F}}$ and that $E$ has good ordinary reduction at all places above $p$. Then we have the inclusion between ideals in $\Lambda_{\mathcal{F}, \mathcal{W}_{p}}$

$$
\left(\operatorname{char}_{\Lambda_{\mathcal{F}}} \operatorname{Sel}_{\mathcal{F}_{\infty}}(E)\right) \subset\left(L_{p}\left(E / \mathcal{F}_{\infty}\right)\right) .
$$

From the above theorem and control theorems (cf. [Gre99, Thm. 1.2, Thm. 4.1]), we can deduce the following consequence which provides evidence to the Birch and Swinnerton-Dyer conjecture.

Corollary 1. Suppose that $p \nmid 6 \cdot h_{\mathcal{K}}^{-} \cdot D_{\mathcal{F}}$ and that $E$ has good ordinary reduction at all places above $p$. Then

(a) If $L\left(E_{/ \mathcal{F}}, 1\right)=0$, then the p-primary Selmer group $\operatorname{Sel}_{\mathcal{F}}(E)$ has positive $\mathbf{Z}_{p}$-corank.

(b) If $L\left(E_{/ \mathcal{F}}, 1\right) \neq 0$, then

$$
\operatorname{length}_{\mathbf{Z}_{p}}\left(\operatorname{Sel}_{\mathcal{F}}(E)\right) \geq \operatorname{ord}_{p}\left(\frac{L\left(E_{/ \mathcal{F}}, 1\right)}{\Omega_{E}}\right),
$$

where $\Omega_{E}$ is the period of a Néron differential of $E$ over $\overline{\mathbf{Z}}_{(p)}$.

Remark. If $W(E / \mathcal{F})=-1$, part (a) is a consequence of the Selmer parity conjecture proved by Nekováŕ [Nek06, Cor. 12.2.10]. Our different approach provides a constructive proof of this fact.

Iwasawa main conjecture for CM fields. We prove Theorem 1 by establishing a divisibility result towards the Iwasawa main conjecture for CM fields, which we will describe after introducing some notation. Let $\mathcal{K}$ be a totally imaginary quadratic extension of $\mathcal{F}$ and assume that $\mathcal{K}$ is $p$-ordinary; namely, every prime of $\mathcal{F}$ above $p$ splits in $\mathcal{K}$. Let $\Sigma$ be a $p$-ordinary CM-type of $\mathcal{K}$ (see $₫ 1.4$ ). Let $S_{p}^{\mathcal{K}}$ be the set of $p$-adic places of $\mathcal{K}$. Fix an embedding $\iota_{p}: \overline{\mathbf{Q}} \hookrightarrow \mathbf{C}_{p}$. Then $\Sigma$ and $\iota_{p}$ give rise to $p$-adic CM-type $\Sigma_{p}$, which is a subset of $S_{p}^{\mathcal{K}}$ such that $\Sigma_{p}$ and its complex conjugation $\Sigma_{p}^{c}$ give a partition of $S_{p}^{\mathcal{K}}$. Let $d=[\mathcal{F}: \mathbf{Q}]$. Let $\mathcal{K}_{\infty}$ be the compositum of the cyclotomic $\mathbf{Z}_{p}$-extension and the anticyclotomic $\mathbf{Z}_{p}^{d}$-extension of $\mathcal{K}$. If we assumed Leopoldt's conjecture for $\mathcal{K}$, then $\mathcal{K}_{\infty}$ would be the maximal $\mathbf{Z}_{p}^{d+1}$-extension of $\mathcal{K}$. Let $\Gamma_{\mathcal{K}}$ be the Galois group $\operatorname{Gal}\left(\mathcal{K}_{\infty} / \mathcal{K}\right)$, which is a free $\mathbf{Z}_{p^{-}}$ module of rank $d+1$. Let $\mathcal{K}^{\prime} \supset \mathcal{K}\left(\mu_{p}\right)$ be a finite abelian extension of $\mathcal{K}$ which is linear disjoint from $\mathcal{K}_{\infty}$ and let $\Delta=\operatorname{Gal}\left(\mathcal{K}^{\prime} / \mathcal{K}\right)$. Let $\psi: \Delta \rightarrow \mathbf{C}_{p}^{\times}$be a character of $\Delta$, which is called a branch character. Let $\mathcal{W}_{p}[\psi]$ be the ring generated by the 
values of $\psi$ over $\mathcal{W}_{p}$. Denote by $\Lambda=\mathcal{W}_{p}[\psi] \llbracket \Gamma_{\mathcal{K}} \rrbracket$ the $(d+1)$-variable Iwasawa algebra over $\mathcal{W}_{p}[\psi]$.

Let $\mathcal{K}_{\infty}^{\prime}=\mathcal{K}^{\prime} \mathcal{K}_{\infty}$ and let $M_{\Sigma}$ be the maximal $p$-abelian $\Sigma_{p}$-ramified extension of $\mathcal{K}_{\infty}^{\prime}$ and let $X_{\Sigma}$ be the Galois group $\operatorname{Gal}\left(M_{\Sigma} / \mathcal{K}_{\infty}^{\prime}\right)$. Then $M_{\Sigma}$ is also Galois over $\mathcal{K}$, and $\Delta$ acts on $X_{\Sigma}$ by the usual conjugation action. Define the Iwasawa module $X_{\Sigma}^{(\psi)}$ to be the maximal $\psi$-isotypic quotient of $X_{\Sigma}$. By [HT94, Thm. 1.2.2], $X_{\Sigma}^{(\psi)}$ is a finitely generated torsion $\Lambda$-module. Therefore, to $X_{\Sigma}^{(\psi)}$ we can attach a characteristic power series $F_{\psi, \Sigma} \in \Lambda$. On the other hand, it follows from Katz Kat78 and Hida-Tilouine [HT93, Thm. II] that to $(\psi, \Sigma)$ we attach a primitive $(d+1)$-variable $p$-adic Hecke $L$-function $L_{\psi, \Sigma} \in \Lambda$, which interpolates $p$-adically the algebraic part of critical Hecke $L$-values for $\psi$ twisted by characters of $\Gamma_{\mathcal{K}}$ and satisfies the functional equation. We can state the $(d+1)$-variable main conjecture for CM fields as follows ( $c f$. [HT94, Main conjecture, page 90]).

Conjecture 2 (Iwasawa main conjecture for CM fields). We have the following equality between ideals in $\Lambda$ :

$$
\left(F_{\psi, \Sigma}\right)=\left(L_{\psi, \Sigma}\right)
$$

The significance of the main conjecture originates from the applications to the Birch and Swinnerton-Dyer conjecture for CM elliptic curves (see CG83 and Rub91 for the case $\mathcal{F}=\mathbf{Q}$ ). When $\mathcal{F}=\mathbf{Q}$, this conjecture is a theorem of Rubin Rub91 combined with Yager's construction of $p$-adic $L$-functions for imaginary quadratic fields Yag82. Rubin uses the technique of an Euler system constructed from elliptic units to bound the size of $X_{\Sigma}^{(\psi)}$ in terms of $L$-values. In other words, he proves the divisibility $\left(F_{\psi, \Sigma}\right) \supset\left(L_{\psi, \Sigma}\right)$, and then appeals to the class number formula to conclude the equality. For general CM fields, the Euler system technique seems not applicable currently. In this article, instead of controlling the size of $X_{\Sigma}^{(\psi)}$ we construct sufficiently many elements in $X_{\Sigma}^{(\psi)}$ in terms of $L$-values with the technique of congruences among modular forms on the unitary group of degree three. Namely, we prove the reverse divisibility relation $\left(F_{\psi, \Sigma}\right) \subset\left(L_{\psi, \Sigma}\right)$. To state our result precisely, we need to introduce some notation. Let $\mathfrak{m}_{\Lambda}$ be the maximal ideal of $\Lambda$. For a number field $L$, we let $G_{L}=\operatorname{Gal}(\overline{\mathbf{Q}} / L)$ and let $\omega_{L}: G_{L} \rightarrow \mathbf{Z}_{p}^{\times}$be the Teichmüller character. Let $D_{\mathcal{K} / \mathcal{F}}$ be the relative discriminant of $\mathcal{K} / \mathcal{F}$ and let $\mathfrak{c}(\psi)$ be the prime-to- $p$ conductor of the branch character $\psi$. Let $\psi_{+}: G_{\mathcal{F}}^{a b} \rightarrow \mathbf{C}_{p}^{\times}$ be the composition $\psi \circ V$, where $V: G_{\mathcal{F}}^{a b} \rightarrow G_{\mathcal{K}}^{a b}$ is the Verschiebung map.

Theorem 11 is a consequence of the following divisibility result in the $(d+1)$ variable main conjecture for CM fields and the control theorems of Selmer groups for CM elliptic curves due to Perrin-Riou PR84.

Theorem 2. Suppose that

(1) $p \nmid 3 \cdot h_{\mathcal{K}}^{-} \cdot D_{\mathcal{F}} \cdot \sharp(\Delta)$,

(2) $\psi$ is unramified at $\Sigma_{p}^{c}$, and $\psi \omega_{\mathcal{K}}^{-a}$ is unramified at $\Sigma_{p}$ for some integer $a \not \equiv 2(\bmod p-1)$.

Then we have the following inclusion between ideals of $\Lambda$ :

$$
\left(F_{\psi, \Sigma}\right) \subset\left(L_{\psi, \Sigma}\right) \text {. }
$$

Remark. The assumption (2) is in particular used to make some twist of $\psi$ unramified at $p$, which is necessary for the application of results on the non-vanishing 
modulo $p$ of Hecke $L$-values in the proof of non-vanishing modulo $p$ of Eisenstein series. It is possible to weaken this assumption if [Hid04a, Prop. 2.8] could be improved.

Thanks to the works of Hida and Rubin, Theorem2 is sufficient to prove the main conjecture when $\psi$ is anticyclotomic or obtained from the restriction of a Galois character of an imaginary quadratic field. By Hida's solution to the anticyclotomic main conjecture in Hid06 and Hid09b, Theorem 2 implies the main conjecture for certain anticyclotomic branch characters.

Corollary 2. Suppose that $p \nmid 3 \cdot h_{\mathcal{K}}^{-} \cdot D_{\mathcal{F}} \cdot \sharp(\Delta)$ and that

(H1) $\psi$ is anticyclotomic,

(H2) the local character $\psi_{w}$ is unramified and non-trivial for every $w \in \Sigma_{p}$,

(H3) $\left.\psi\right|_{G_{\mathcal{K}\left(\sqrt{p^{*}}\right)}} \neq 1, p^{*}=(-1)^{\frac{p-1}{2}} p$.

Then we have the following equality between ideals of $\Lambda$ :

$$
\left(F_{\psi, \Sigma}\right)=\left(L_{\psi, \Sigma}\right)
$$

Combined with Rubin's two-variable main conjecture for imaginary quadratic fields Rub91, Theorem 2 yields the following main conjecture by the same argument in [Hid07, Thm. 5.7].

Corollary 3. In addition to the assumptions (1-2) in Theorem 2, suppose further that

(R1) $\mathcal{K}=\mathcal{F} \mathcal{M}$, where $\mathcal{M}$ is an imaginary quadratic field in which $p$ splits,

(R2) $\Sigma$ is the p-ordinary CM-type of $\mathcal{K}$ obtained by extending the inclusion $\iota_{\infty}$ : $\mathcal{M} \hookrightarrow \mathbf{C}$,

(R3) $\mathcal{K}^{\prime}$ is abelian over $\mathcal{M}$ and $p \nmid\left[\mathcal{K}^{\prime}: \mathcal{M}\right]$.

Then we have the following equality between ideals of $\Lambda$ :

$$
\left(F_{\psi, \Sigma}\right)=\left(L_{\psi, \Sigma}\right) .
$$

The main conjecture from Greenberg's point of view. We give a different formulation of Conjecture 2 proposed by R. Greenberg in the context of Galois representations (Gre94]). Let $S_{\psi}$ be the set of places dividing $\mathfrak{c}(\psi)$ and let $S \supset S_{\psi}$ be a finite set of prime-to- $p$ places in $\mathcal{K}$. Let $\mathcal{K}_{S}$ be the maximal algebraic extension of $\mathcal{K}$ unramified outside $S \cup S_{p}^{\mathcal{K}}$. Define the tautological $\Lambda$-valued Galois character $\varepsilon_{\Lambda}$ by

$$
\begin{aligned}
\varepsilon_{\Lambda}: \operatorname{Gal}\left(\mathcal{K}_{S} / \mathcal{K}\right) & \longrightarrow \Gamma_{\mathcal{K}} \hookrightarrow \Lambda^{\times} \\
g & \left.\longrightarrow g\right|_{\mathcal{K}_{\infty} .}
\end{aligned}
$$

Let $\Psi: \operatorname{Gal}\left(\mathcal{K}_{S} / \mathcal{K}\right) \rightarrow \Lambda^{\times}$be the deformation of $\psi$ defined by $\Psi(g)=\psi(g) \varepsilon_{\Lambda}(g)$. Let $\Lambda^{*}=\operatorname{Hom}_{\text {cont }}\left(\Lambda, \mathbf{Q}_{p} / \mathbf{Z}_{p}\right)$ be the Pontryagin dual of $\Lambda$. We make $\Lambda^{*}$ a discrete $\Lambda$-module by $\lambda s(x)=s(\lambda x)$ for all $\lambda, x \in \Lambda$ and $s \in \Lambda^{*}$. The $\Lambda$-adic Selmer group associated to $(\Psi, \Sigma)$ is defined by

$$
\operatorname{Sel}_{\mathcal{K}}(\Psi, \Sigma):=\operatorname{ker}\left\{H^{1}\left(\mathcal{K}_{S} / \mathcal{K}, \Psi \otimes_{\Lambda} \Lambda^{*}\right) \rightarrow \prod_{w \in S \cup \Sigma_{p}^{c}} H^{1}\left(I_{w}, \Psi \otimes_{\Lambda} \Lambda^{*}\right)\right\},
$$


where $I_{w}$ is the inertia group in a local decomposition group of $G_{\mathcal{K}}$ at $w$. Define the $S$-imprimitive Selmer group by

$$
\operatorname{Sel}_{\mathcal{K}}^{S}(\Psi, \Sigma):=\operatorname{ker}\left\{H^{1}\left(\mathcal{K}_{S} / \mathcal{K}, \Psi \otimes_{\Lambda} \Lambda^{*}\right) \rightarrow \prod_{w \in \Sigma_{p}^{c}} H^{1}\left(I_{w}, \Psi \otimes_{\Lambda} \Lambda^{*}\right)\right\} .
$$

Let $\mathrm{ht}_{1}(\Lambda)$ be the set of height one prime ideals in $\Lambda$. If $\mathcal{S}$ is a cotorsion and cofinitely generated discrete $\Lambda$-module and $P \in \mathrm{ht}_{1}(\Lambda)$, we denote by $\mathcal{S}_{P}^{*}=\left(\mathcal{S}^{*}\right) \otimes_{\Lambda} \Lambda_{P}$ the localization of the Pontryagin dual of $\mathcal{S}$ at $P$ and put

$$
\ell_{P}(\mathcal{S}):=\operatorname{length}_{\Lambda_{P}}\left(\mathcal{S}_{P}^{*}\right)
$$

We write $L_{p}(\Psi, \Sigma)$ for the Katz $p$-adic $L$-function $L_{\psi, \Sigma}$. Conjecture 2 can be reformulated as the following main conjecture for the one dimensional $\Lambda$-adic Galois representation $\Psi$.

Conjecture 3 (Main conjecture). For every $P \in \mathrm{ht}_{1}(\Lambda)$, we have the equality

$$
\ell_{P}\left(\operatorname{Sel}_{\mathcal{K}}(\Psi, \Sigma)\right)=\operatorname{ord}_{P}\left(L_{p}(\Psi, \Sigma)\right) .
$$

We shall consider the dual version of the above main conjecture, which has the advantage of incorporating imprimitive $p$-adic $L$-functions and Selmer groups. Define the $S$-imprimitive $p$-adic $L$-function $L_{p}^{S}(\Psi, \Sigma)$ for $S$ by

$$
L_{p}^{S}(\Psi, \Sigma)=L_{p}(\Psi, \Sigma) \cdot \prod_{w \in S-S_{\psi}}\left(1-\Psi\left(\operatorname{Frob}_{w}\right)\right) .
$$

Here Frob $_{w}$ is the geometric Frobenious. Let $\varepsilon$ be the $p$-adic cyclotomic character of $G_{\mathcal{K}}$ and let $\Psi^{D}$ be the character defined by $\Psi^{D}(g)=\Psi^{-1} \varepsilon(g)$. The dual Selmer group for $(\Psi, \Sigma)$ is the Selmer group associated to $\left(\Psi^{D}, \Sigma^{c}\right)$ defined by

$$
\operatorname{Sel}_{\mathcal{K}}^{S}\left(\Psi^{D}, \Sigma^{c}\right):=\operatorname{ker}\left\{H^{1}\left(\mathcal{K}_{S} / \mathcal{K}, \Psi^{D} \otimes_{\Lambda} \Lambda^{*}\right) \rightarrow \prod_{w \in \Sigma_{p}} H^{1}\left(I_{w}, \Psi^{D} \otimes_{\Lambda} \Lambda^{*}\right)\right\} .
$$

We will prove the following theorem, which implies Theorem 2 by the functional equation of Selmer groups for CM fields [Hsi10.

Theorem 3. With the same assumptions (1-2) in Theorem 2, for every $P \in \mathrm{ht}_{1}(\Lambda)$ we have the inequality

$$
\ell_{P}\left(\operatorname{Sel}_{\mathcal{K}}^{S}\left(\Psi^{D}, \Sigma^{c}\right)\right) \geq \operatorname{ord}_{P}\left(L_{p}^{S}(\Psi, \Sigma)\right) .
$$

Eisenstein congruence. Our main tool is the Eisenstein congruence on unitary groups. The application of Eisenstein congruence to Iwasawa theory was first introduced by Ribet in Rib76, in which he uses the congruences between Eisenstein series and cusp forms on GL(2) over $\mathbf{Q}$ to obtain a proof of the converse of Herbrand's theorem. This approach was further exploited by Mazur and Wiles MW84 in their proof of classical Iwasawa main conjecture, and by Wiles Wil90. in his elegant proof of Iwasawa's main conjecture for totally real fields through the systematic use of Hida theory. Meanwhile, parallel to Eisenstein congruence, Hida and Tilouine studied CM congruence for GL(2) over totally real fields extensively, and in [HT94] they proved a divisibility result for the anticyclotomic main conjecture for CM fields. In Urb01 and Urb06, E. Urban proved the main conjecture for 
adjoint modular representation by Eisenstein congruence on GSp(4). In his joint work with C. Skinner [SU13], they prove the main conjecture for GL(2) by Eisenstein congruence on $U(2,2)$.

The proof of our Theorem 3 is based on a study of Eisenstein congruence on $U(2,1)$, the quasi-split unitary group of degree three. The use of Eisenstein congruence for $U(2,1)$ to study one-sided divisibility in the main conjecture for $\mathrm{CM}$ fields was initiated by F. Mainardi in his thesis Mai04 under the supervision of J. Tilouine and E. Urban. In Mai08] he defines the Eisenstein ideal of the cuspidal ordinary topological Hecke algebra $\mathbf{B}$, i.e. the Hecke algebra acting on the ordinary cuspidal Betti cohomology groups, and proves that the characteristic power series of Selmer groups for CM fields is divisible by the Eisenstein ideal in $\mathbf{B}$ under some technical assumptions. In this article, we introduce the ideal of Eisenstein congruence $\operatorname{Eis}(\Psi, S) \subset \Lambda$ (Def. 7.19), which measures the congruence between Hida families of Eisenstein series and cusp forms on $U(2,1)$, and we first prove Theorem 3 for every $P \in \mathrm{ht}_{1}(\Lambda)$ which is not exceptional (Def. 8.13) by establishing the following two inequalities:

$(\mathrm{L} \mid \mathrm{E}) \operatorname{ord}_{P}(\operatorname{Eis}(\Psi, S)) \geq \operatorname{ord}_{P}\left(L_{p}^{S}(\Psi, \Sigma)\right)$,

$(\mathrm{E} \mid \mathrm{S}) \ell_{P}\left(\operatorname{Sel}_{\mathcal{K}}^{S}\left(\Psi^{D}, \Sigma^{c}\right)\right) \geq \operatorname{ord}_{P}(\operatorname{Eis}(\Psi, S))$.

Let us explain the strategy briefly. To show the first inequality $(\mathrm{L} \mid \mathrm{E})$, we have to work with Hida families for unitary groups. The first step is to construct a $\Lambda$-adic Hida family of Eisenstein series $\mathcal{E}^{\text {ord }}$ (a $p$-adic measure with values in the space of $p$-adic ordinary modular forms) on $U(2,1)$ with the optimal constant term, namely a product of the Katz $p$-adic $L$-function $L_{p}^{S}(\Psi, \Sigma)$ and a Tate twist of the $S$-imprimitive Deligne-Ribet $p$-adic $L$-function $L_{D R}^{S}$ associated to the character $\psi_{+} \tau_{\mathcal{K} / \mathcal{F}} \omega_{\mathcal{F}}^{-1}\left(\tau_{\mathcal{K} / \mathcal{F}}\right.$ is the quadratic character of $\left.\mathcal{K} / \mathcal{F}\right)$. The idea of this construction is based on our previous work [Hsi11, which we describe briefly as follows. We begin with the construction of a good p-adic Siegel-Eisenstein series $\mathcal{E}_{2,2}$ on $U(2,2)$. Applying the pull-back formula, we obtain an Eisenstein series $\mathcal{E}$ on $U(2,1)$ by pulling back this Siegel-Eisenstein series to $U(2,1)$ via a suitable embedding $U(2,1) \times U(1) \hookrightarrow U(2,2)$. The desired ordinary Eisenstein series is constructed by taking the ordinary projection $\mathcal{E}^{\text {ord }}$ of $\mathcal{E}$. The idea of using the pull-back formula was suggested to the author by E. Urban, and has been used in Urb06 and [SU13 to construct a Hida family of Eisenstein series on GSp(4) and $U(2,2)$ respectively.

The construction of our degree two Siegel-Eisenstein series $\mathcal{E}_{2,2}$ is inspired by the construction of Harris, Li and Skinner [HLS06. However, their Eisenstein series does not quite fit for our purpose since the ordinary projection of the pull-back of this Eisenstein series is zero. A heuristic reason is that the Fourier coefficients of their Eisenstein series are only supported in the integral Hermitian matrices which are non-degenerate modulo $p$. Therefore, we have to modify their construction to meet the ordinary condition, which complicates the computation of the constant term of the ordinary projection of of the pull-back of $\varepsilon_{2,2}$. To resolve this, we apply the techniques in [Hsi11, §6].

Second, to make congruences between Hecke eigenvalues of Eisenstein series and cusp forms for $U(2,1)$ modulo the constant term, we have to show that the Hida family of Eisenstein series $\mathcal{E}^{\text {ord }}$ is co-prime to the constant term (or even nonvanishing modulo $\mathfrak{m}_{\Lambda}$ ). This is usually the most difficult step in the approach of 
Eisenstein congruence. In the case of $U(2,2)$ [SU13, this is achieved by showing the non-vanishing modulo $p$ of a very clever linear combination of Fourier coefficients of certain Klingen-Eisenstein series. In our situation, we show directly that some Fourier-Jacobi coefficient of $\mathcal{E}^{\text {ord }}$ is non-vanishing modulo $\mathfrak{m}_{\Lambda}$, making heavy use of the theory of Shintani on primitive Fourier-Jacobi coefficients of automorphic forms on $U(2,1)$ Shi79. Our idea is to introduce an auxiliary Eisenstein series $E^{\circ}$, whose Fourier-Jacobi coefficients are manageable, and show that the non-vanishing modulo $p$ of $p$-primitive Fourier-Jacobi coefficients of $E^{\circ}$ and $\varepsilon^{o r d}$ are equivalent (see Prop. 7.8). The Fourier-Jacobi coefficients of $E^{\circ}$ are essentially a product of two Hecke $L$-values for CM fields thanks to the works of Murase and Sugano (MS00, MS02]) generalizing Shintani's theory and the calculation of Tonghai Yang on period integrals of theta functions. The problem is thus reduced to the nonvanishing modulo $p$ of these Hecke $L$-values, which is addressed in Hid04a and our previous work Hsi12. Using the non-vanishing of $\mathcal{E}^{\text {ord }}\left(\bmod \mathfrak{m}_{\Lambda}\right)$ combined with Hida theory for unitary groups, we are able to construct a non-trivial Hida family of cusp forms congruent to the Eisenstein series $\mathcal{E}^{\text {ord }}$ modulo $p$-adic $L$-functions, which leads to the inequality $(\mathrm{L} \mid \mathrm{E})$.

To show the second inequality $(\mathrm{E} \mid \mathrm{S})$, we need to construct sufficiently many elements in the Selmer group in terms of the Eisenstein ideal. This can be done by the technique of lattice construction due to E. Urban in [Urb01]. In our case, a variant of the inequality $(\mathrm{E} \mid \mathrm{S})$ in the context of the topological cuspidal ordinary Hecke algebra has been worked out by Mainardi Mai08. Working with the coherent cuspidal ordinary Hecke algebra instead, we achieve the inequality $(\mathrm{E} \mid \mathrm{S})$.

There remains the case when $P$ is exceptional. These are precisely common divisors of the Katz $p$-adic $L$-function $L_{\psi, \Sigma}$ and the $S$-imprimitive Deligne-Ribet $p$-adic $L$-function $L_{D R}^{S}$. We have trouble proving $(\mathrm{E} \mid \mathrm{S})$ for exceptional primes $P$ in general unless $\operatorname{ord}_{P}\left(L_{\psi, \Sigma}\right) \leq 1$. Nonetheless, if $\Delta$ has order prime to $p$, then the results of Hida [Hid10] and the author [Hsi13] on the vanishing of the $\mu$-invariant of anticyclotomic $p$-adic $L$-functions for CM fields imply that there is no exceptional prime unless $\psi_{+}=\tau_{\mathcal{K} / \mathcal{F}} \omega_{\mathcal{F}}$ and $W_{\Sigma}(\psi) \equiv-1\left(\bmod \mathfrak{m}_{\Lambda}\right)$, where $W_{\Sigma}(\psi) \in \Lambda^{\times}$ is the root number in the functional equation of the Katz $p$-adic function $L_{\psi, \Sigma}$, and in this particular case, the only possible exceptional prime is the pole $P_{e}$ of $L_{D R}$ and $\operatorname{ord}_{P_{e}}\left(L_{\psi, \Sigma}\right)=1$ thanks to a recent result of A. Burungale Bur13 on the $\mu$-invariant of the cyclotomic derivative of Katz $p$-adic $L$-functions attached to self-dual characters with root number -1 .

Structure of this article. This paper is organized as follows.

In $\$ 1$ we fix notation and definitions through this article. In $\$ 2$ and $\$ 3$, we review the general theory of Shimura varieties associated to unitary groups over totally real fields and the theory of Katz-Hida geometric modular forms. In $\$ 4$ we extend Hida theory to include Eisenstein series on unitary groups $U(r, 1)$ over totally real fields. We prove the classicality and the control theorem for $p$-adic ordinary modular forms. Hida theory provides the framework for the study of the congruence among modular forms. In particular, the fundamental exact sequence in Theorem 4.26 (cf. [Urb06, Thm. 2.4.19] and [SU13, Thm. 6.3.10]) is crucial to make congruence between Eisenstein series and cusp forms modulo constant terms in $\$ 7$ Hida developed his theory for cusp forms in great generality ([Hid02] and [Hid04b]), and moreover he establishes an axiomatic control theorem for automorphic sheaves 
on Shimura varieties of PEL-type using several standard results from the theory of the minimal and toroidal compactifications of Shimura varieties of PEL-type which has been worked out by K.-W. Lan Lan08 in full detail. Hida theory for modular forms on $U(n, n)_{/ \mathbf{Q}}$ has been carried out in [SU13], and the proofs therein work for $U(r, 1)_{/ \mathbf{Q}}$ as well. However, due to the appearance of non-torsion units in $\mathcal{O}_{\mathcal{F}}^{\times}$, one requires a slight modification for the base change property in the totally real case (see 4.17$)$.

In $\$ 5$ and $₫ 6$, we construct the desired ordinary $p$-adic Eisenstein series on $U(2,1)$ and make an explicit calculation on its constant terms (Prop. 6.6). In \$7, we show our ordinary $p$-adic Eisenstein series is non-vanishing modulo $p$ (Prop. 7.13) and construct congruences between cusp forms and Eisenstein series on $U(2,1)$, which leads to our first inequality (L|S) (Cor. 7.20).

Finally in 88 by the technique of lattice construction we prove the second inequality $(\mathrm{E} \mid \mathrm{S})$ (Theorem 8.14). A variant of Mainardi's work in the context of the coherent Hecke algebras is carried out in $\$ 8.3$ and $\$ 8.4$ The applications to the main conjectures for CM elliptic curves and CM fields are given in $\S 8.6$.

\section{Notation AND CONVEntions}

1.1. Throughout $\mathcal{F}$ is a totally real field of degree $d$ over $\mathbf{Q}$ and $\mathcal{K}$ is a totally imaginary quadratic extension of $\mathcal{F}$. Denote by $c$ the complex conjugation, the unique non-trivial element in $\operatorname{Gal}(\mathcal{K} / \mathcal{F})$. Denote by $\mathbf{a}=\operatorname{Hom}(\mathcal{F}, \mathbf{C})$ the set of archimedean places of $\mathcal{F}$ and by $\mathbf{h}$ the set of finite places of $\mathcal{F}$. Let $D_{\mathcal{K} / \mathcal{F}}\left(\right.$ resp. $\left.\mathcal{D}_{\mathcal{K} / \mathcal{F}}\right)$ be the relative discriminant (resp. different) of $\mathcal{K} / \mathcal{F}$ and $S_{\mathcal{K} / \mathcal{F}}=\left\{v \in \mathbf{h}|v| D_{\mathcal{K} / \mathcal{F}}\right\}$.

Henceforth we fix an odd rational prime $p$ which is unramified in $\mathcal{F}$ and assume the following ordinary condition:

$$
\text { Every prime of } \mathcal{F} \text { above } p \text { splits in } \mathcal{K} \text {. }
$$

This condition implies that $p$ is unramified in $\mathcal{K}$. We denote by $S_{p}$ the set of places in $\mathcal{F}$ lying above $p$. We choose once and for all an embedding $\iota_{\infty}: \overline{\mathbf{Q}} \hookrightarrow \mathbf{C}$ and an isomorphism $\iota: \mathbf{C} \stackrel{\sim}{\rightarrow} \mathbf{C}_{p}$, where $\mathbf{C}_{p}$ is the completion of an algebraic closure of $\mathbf{Q}_{p}$. Let $\iota_{p}=\iota \iota_{\infty}: \overline{\mathbf{Q}} \hookrightarrow \mathbf{C}_{p}$ be their composition. Let $\overline{\mathbf{Z}}_{p}$ be the $p$-adic completion of the ring $\overline{\mathbf{Z}}$ of algebraic integers in $\mathbf{C}_{p}$ and let $\mathfrak{m}_{p}$ be the maximal ideal of $\overline{\mathbf{Z}}_{p}$ and let $\mathfrak{m}:=\iota^{-1}\left(\mathfrak{m}_{p}\right)$. Every number field $L$ will be regarded as a subfield in $\mathbf{C}$ (resp. $\left.\mathbf{C}_{p}\right)$ via $\iota_{\infty}\left(\right.$ resp. $\left.\iota_{p}\right)$ and $\operatorname{Hom}(L, \overline{\mathbf{Q}})=\operatorname{Hom}\left(L, \mathbf{C}_{p}\right)$.

If $L$ is a finite extension of $\mathbf{Q}_{p}$ or a number field, we denote by $\mathcal{O}_{L}$ the ring of integers of $L$. If $L^{\prime}$ is a quadratic extension of $L$, denote by $\tau_{L^{\prime} / L}$ the quadratic character associated to $L^{\prime} / L$. If $L$ is number field, let $G_{L}=\operatorname{Gal}(\overline{\mathbf{Q}} / L)$ be the absolute Galois group. Let $\mathbf{A}_{L}$ be the adele ring of $L$ and $\mathbf{A}_{L, f}$ be the finite part of $\mathbf{A}_{L}$. For brevity, we shall write $O=\mathcal{O}_{\mathcal{F}}$ throughout this article.

We often write $w$ for a place of $\mathcal{K}$ and $v$ for a place of $\mathcal{F}$. Write $\mathcal{K}_{w}$ (resp. $\mathcal{F}_{v}$ ) for the completion of $\mathcal{K}$ (resp. $\mathcal{F}$ ) at $w$ (resp. $v$ ) and $\mathcal{F}_{p}$ for $\mathcal{F} \otimes_{\mathbf{Q}} \mathbf{Q}_{p}$. Denote by $\varpi_{w}\left(\right.$ resp. $\left.\varpi_{v}\right)$ a uniformizer of $\mathcal{K}_{w}\left(\right.$ resp. $\left.\mathcal{F}_{v}\right)$. We also write $\mathcal{K}_{v}$ for $\mathcal{K} \otimes_{\mathcal{F}} \mathcal{F}_{v}$ and $O_{v}\left(\right.$ resp. $\left.O_{p}\right)$ for $\mathcal{O}_{\mathcal{F}_{v}}\left(\right.$ resp. $\left.O \otimes \mathbf{z} \mathbf{Z}_{p}\right)$.

Denote by $\hat{\mathbf{Z}}$ the finite completion of $\mathbf{Z}$. If $M$ is an abelian group, let $\widehat{M}=$ $M \otimes \mathbf{z} \hat{\mathbf{Z}}$. If $L$ is a number field, $\widehat{L}=\mathbf{A}_{L, f}$ and $\widehat{\mathcal{O}}_{L}=\prod_{v<\infty} \mathcal{O}_{L_{v}}$. 
1.2. For a finite set $\square$ of rational primes, we define $\mathbf{Z}_{(\square)}$ by

$$
\mathbf{Z}_{(\square)}=\left\{\frac{a}{b} \in \mathbf{Q} \mid b \mathbf{Z}+q \mathbf{Z}=\mathbf{Z} \text { for all } q \in \square\right\} .
$$

By definition, $\mathbf{Z}_{(\square)}=\mathbf{Q}$ if $\square$ is empty. If $R$ is a $\mathbf{Z}$-algebra, we let $R_{(\square)}=R \otimes_{\mathbf{Z}} \mathbf{Z}_{(\square)}$. When $\square=\{p\}$, we write $R_{(\square)}$ as $R_{(p)}$. Denote by $\mathbf{Z}_{+}$the set of positive integers. Put

$$
O_{(\square),+}=\left\{a \in O \otimes \mathbf{z} \mathbf{Z}_{(\square)} \mid a \text { is totally positive }\right\} .
$$

If $R$ is a ring, we denote by $S C H_{/ R}$ the category of $R$-schemes and by $S E T S$ the category of sets. If $X$ is a scheme over $\mathcal{F}$ (resp. $O$ ), $\mathrm{R}_{\mathcal{F} / \mathbf{Q}} X\left(\operatorname{resp} . \mathrm{R}_{O / \mathbf{Z}} X\right)$ is the restriction of scalar of $X$ from $\mathcal{F}$ (resp. $O$ ) to $\mathbf{Q}$ (resp. $\mathbf{Z}$ ).

If $R$ is an $O$-algebra, the complex conjugation $c$ induces an involution on $R \otimes_{O} \mathcal{K}$ by $r \otimes x \mapsto r \otimes c(x)$. Define the $n \times n$ Hermitian matrices $\mathcal{H}_{n}(R)$ over $R \otimes_{O} \mathcal{K}$ by

$$
\mathcal{H}_{n}(R)=\left\{g \in M_{n}\left(R \otimes_{O} \mathcal{K}\right) \mid g=g^{*}\right\},
$$

where $g^{*}=c\left(g^{t}\right)$ and $g^{t}$ is the transpose of $g$. When $n=1$ and $g \in R \otimes_{O} \mathcal{K}$, we sometimes write $\bar{g}$ for $g^{*}$. If $g \in \mathrm{GL}_{n}\left(R \otimes_{O} \mathcal{K}\right)$, we write $g^{-*}$ and $g^{-t}$ for $\left(g^{*}\right)^{-1}$ and $\left(g^{t}\right)^{-1}$ respectively. We denote by $B_{n}(R)$ the upper triangular subgroup of $\mathrm{GL}_{n}(R)$. Let $T_{n}(R)$ be the diagonal torus of $B_{n}(R)$ and let $N_{n}(R)$ be the unipotent radical of $B_{n}(R)$.

1.3. Characters. Let $\mathrm{I}_{\mathcal{K}}=\operatorname{Hom}(\mathcal{K}, \overline{\mathbf{Q}})$. For $w \in S_{p}^{\mathcal{K}}$, we put

$$
\mathrm{I}_{w}=\left\{\sigma_{p} \in \operatorname{Hom}\left(\mathcal{K}, \mathbf{C}_{p}\right) \mid \sigma_{p} \text { induces } w\right\}=\operatorname{Hom}\left(\mathcal{K}_{w}, \mathbf{C}_{p}\right) .
$$

We shall identity $\mathrm{I}_{\mathcal{K}}$ with $\bigsqcup_{w} \mathrm{I}_{w}$ by $\sigma \mapsto \sigma_{p}:=\iota_{p} \circ \sigma$.

Let $\chi: \mathbf{A}_{\mathcal{K}}^{\times} / \mathcal{K}^{\times} \rightarrow \mathbf{C}^{\times}$be an arithmetic Hecke character of $\mathcal{K}^{\times}$. We say that $\chi$ has infinity type $\kappa=\sum_{\sigma \in \mathrm{I}_{\mathcal{K}}} \kappa_{\sigma} \sigma \in \mathbf{Z}\left[\mathrm{I}_{\mathcal{K}}\right]$ if

$$
\chi_{\infty}(\alpha)=\iota_{\infty}\left(\alpha^{\kappa}\right):=\prod_{\sigma \in \mathrm{I}_{\mathcal{K}}} \sigma(\alpha)^{\kappa_{\sigma}} \text { for all } \alpha \in \mathcal{K}^{\times} .
$$

We can associate $\chi$ to a unique $p$-adic Hecke character $\widehat{\chi}: \mathbf{A}_{\mathcal{K}}^{\times} / \mathcal{K}^{\times} \rightarrow \overline{\mathbf{Z}}_{p}^{\times}$defined by

$$
\widehat{\chi}(z)=\iota_{p}\left(\chi(z) \prod_{\sigma \in \mathrm{I}_{\mathcal{K}}} z_{\sigma}^{-\kappa_{\sigma}}\right) \prod_{w \mid p} \prod_{\sigma_{p} \in \mathrm{I}_{w}} \sigma_{p}\left(z_{w}\right)^{\kappa_{\sigma}} .
$$

We call $\widehat{\chi}$ the $p$-adic avatar of $\chi$, whereas $\chi$ is the complex avatar of $\widehat{\chi}$.

Denote by $\operatorname{rec}_{\mathcal{K}}: \mathbf{A}_{\mathcal{K}}^{\times} / \mathcal{K}^{\times} \rightarrow G_{\mathcal{K}}^{a b}$ the geometrically normalized reciprocity law which takes $\operatorname{rec}_{\mathcal{K}}\left(\varpi_{w}\right)=$ Frob $_{w}$, where $\operatorname{Frob}_{w}$ is the geometric Frobenius at $w$. Throughout this article, every character of $G_{\mathcal{K}}$ implicitly will be regarded as a Hecke character of $\mathcal{K}^{\times}$via $\operatorname{rec}_{\mathcal{K}}$.

1.4. CM-types. Let $\Sigma$ be a CM-type of $\mathcal{K}$, i.e. $\Sigma$ is a subset of $\mathrm{I}_{\mathcal{K}}$ such that

$$
\Sigma \cap \Sigma c=\emptyset \text { and } \Sigma \sqcup \Sigma c=\mathrm{I}_{\mathcal{K}} \text {. }
$$

Denote by $\Sigma_{p}$ the set of places of $\mathcal{K}$ above $p$ induced by $\iota_{p} \circ \sigma$ for $\sigma \in \Sigma$. We further assume $\Sigma$ is a $p$-ordinary CM-type. Namely,

$$
\Sigma_{p} \cap \Sigma_{p} c=\emptyset \text { and } \Sigma_{p} \sqcup \Sigma_{p} c=S_{p}^{\mathcal{K}} .
$$

The existence of a $p$-ordinary CM-type is assured by the assumption (ord). 
Henceforth, we simply write $\Sigma^{c}=\Sigma c$ and $\Sigma_{p}^{c}=\Sigma_{p} c$ and identify the CM-type $\Sigma$ (resp. the $p$-adic CM-type $\Sigma_{p}$ ) with the set $\mathbf{a}$ of archimedean places of $\mathcal{F}$ (resp. the set $S_{p}$ of places of $\mathcal{F}$ above $p$ ).

1.5. Let $\mathcal{K}^{n c}$ be the normal closure of $\mathcal{K}$ in $\overline{\mathbf{Q}}$. To every $\sigma \in \mathrm{I}_{\mathcal{K}}$ we can attach an idempotent $e_{\sigma} \in \mathcal{O}_{\mathcal{K}} \otimes_{\mathbf{Z}} \mathcal{O}_{\mathcal{K}^{n c}}$ such that $a e_{\sigma}=\sigma(a) e_{\sigma}$ for $a \in \mathcal{O}_{\mathcal{K}}$ and

$$
\mathcal{O}_{\mathcal{K}} \otimes \mathbf{Z} \mathcal{O}_{\mathcal{K}^{n c}}=\bigoplus_{\sigma \in \mathrm{I}_{\mathcal{K}}} \mathcal{O}_{\mathcal{K}^{n c}} \cdot e_{\sigma}
$$

For a subset $J$ of $\mathrm{I}_{\mathcal{K}}$, we put $e_{J}=\sum_{\sigma \in J} e_{\sigma}$. Let $e^{+}=e_{\Sigma}$ and $e^{-}=e_{\Sigma^{c}}$. For $w \in S_{p}^{\mathcal{K}}$, let $e_{w}=e_{\mathbf{I}_{w}}$. Since $p$ is unramified in $\mathcal{K}, e_{w}$ belongs to $\mathcal{O}_{\mathcal{K}} \otimes_{\mathbf{z}} \mathbf{Z}_{p}$ and

$$
e_{w}\left(\mathcal{O}_{\mathcal{K}} \otimes \mathbf{z} \mathbf{Z}_{p}\right)=O_{v},
$$

where $v$ is the place of $\mathcal{F}$ lying below $w$. By the definition of $\Sigma_{p}$, we have $e^{+}=$ $\sum_{w \in \Sigma_{p}} e_{w}$ (resp. $\left.e^{-}=\sum_{w \in \Sigma_{p}^{c}} e_{w}\right)$ and $e^{+}\left(\mathcal{O}_{\mathcal{K}} \otimes \mathbf{Z}_{p}\right)=e^{-}\left(\mathcal{O}_{\mathcal{K}} \otimes \mathbf{Z}_{p}\right)=O_{p}$. If $M$ is an $\mathcal{O}_{\mathcal{K}}$-module, we put $M_{p}=M \otimes \mathbf{Z}_{p}$ and

$$
M_{\Sigma}=e^{+}\left(M_{p}\right) \text { and } M_{\Sigma^{c}}=e^{-}\left(M_{p}\right) \text {. }
$$

For $\sigma \in \mathrm{I}_{\mathcal{K}}$, denote by $\mathbf{C}(\sigma)$ the $\mathcal{K} \otimes_{\mathbf{Q}} \mathbf{C}$-module whose underlying space is $\mathbf{C}$ with $\mathcal{K}$-action via $\iota_{\infty} \sigma$. Put

$$
\mathbf{C}(J)=\bigoplus_{\sigma \in J} \mathbf{C}(\sigma)
$$

Let $O_{p}(\Sigma)$ (resp. $\left.O_{p}\left(\Sigma^{c}\right)\right)$ denote the $\mathcal{O}_{\mathcal{K}} \otimes_{\mathbf{Z}} \mathbf{Z}_{p}$-module $O_{p}$ on which $\mathcal{O}_{\mathcal{K}}$ acts through $e_{+}$(resp. $\left.e_{-}\right)$.

1.6. Unitary groups. Let $r$ and $s$ be two non-negative integers such that $r \geq s \geq$ 0 . Let $(W, \vartheta)$ be a skew-Hermitian $\mathcal{K}$-space of dimension $r-s$ with a skew-Hermitian form $\vartheta$. We fix a $\mathcal{K}$-basis $w^{1}, \ldots, w^{r-s}$ of $W$ and assume that $\vartheta\left(w^{i}, w^{j}\right)=a_{i} \delta_{i, j}$. We assume further that $\sqrt{-1} \cdot \sigma\left(a_{i}\right)>0$ and $\iota_{p}\left(\sigma\left(a_{i}\right)\right)$ are $p$-adic units for all $i$ and $\sigma \in \Sigma$. Let $X_{\mathcal{K}}=\bigoplus_{i=1}^{s} \mathcal{K} x^{i}$ and $Y_{\mathcal{K}}=\bigoplus_{i=1}^{s} \mathcal{K} y^{i}$ be $\mathcal{K}$-vector spaces of dimension $s$ and let $\left(V, \vartheta_{r, s}\right)$ be the skew-Hermitian space with the underlying $\mathcal{K}$-vector space $V=Y_{\mathcal{K}} \oplus W \oplus X_{\mathcal{K}}$ and $\vartheta_{r, s}$ the skew-Hermitian form on $V$ defined by

$$
\vartheta_{r, s}=\left[\begin{array}{ccc} 
& & \\
& & \\
\mathbf{1}_{s} & &
\end{array}\right]
$$

with respect to the decomposition $Y_{\mathcal{K}} \oplus W \oplus X_{\mathcal{K}}$. Let $\langle,\rangle_{r, s}: V \times V \rightarrow \mathbf{Q}$ be the alternating pairing defined by $\left\langle v, v^{\prime}\right\rangle_{r, s}=\operatorname{Tr}_{\mathcal{K} / \mathbf{Q}}\left(\vartheta_{r, s}\left(v, v^{\prime}\right)\right)$.

Let $G=G U(V)$ be the group of unitary similitudes attached to the quadratic space $\left(V, \vartheta_{r, s}\right)$. As an algebraic group over $\mathcal{F}$, the $R$-points of $G$ for an $\mathcal{F}$-algebra $R$ are

$$
G(R)=\left\{g \in \mathrm{GL}_{r+s}\left(\mathcal{K} \otimes_{\mathcal{F}} R\right) \mid g \vartheta_{r, s} g^{*}=\nu(g) \vartheta_{r, s} \text { for some } \nu(g) \in R^{\times}\right\} .
$$

The morphism $\nu: G \rightarrow \mathbb{G}_{m / \mathcal{F}}$ is called the similitude map. The unitary group $U(V)$ is defined by

$$
U(V)(R):=\{g \in G U(V)(R) \mid \nu(g)=1\} .
$$

We let $G U(0, r-s)$ be the group of unitary similitudes attached to $(W,-\vartheta)$. 
For $g \in \operatorname{End}_{\mathcal{K}} V$, we let $g^{\vee}$ denote the element in $\operatorname{End}_{\mathcal{K}} V$ such that $\vartheta_{r, s}\left(v g, v^{\prime}\right)=$ $\vartheta_{r, s}\left(v, v^{\prime} g^{\vee}\right), v, v^{\prime} \in V$. Then $g^{\vee}=g^{-1} \nu(g)$ if $g \in G$.

1.7. Standard basis. The basis $\left\{y^{i}, x^{i}\right\}_{i=1, \ldots, s}$ and $\left\{w^{i}\right\}_{i=1, \ldots, r-s}$ is called the standard basis of $V$. The basis $\left\{e_{w} y^{i}, e_{w} w^{i}, e_{w} x^{i}\right\}_{w \in S_{p}^{\mathcal{K}}}$ (resp. $\left\{e_{\sigma} y^{i} \cdot e_{\sigma} w^{i}\right.$, $\left.e_{\sigma} x^{i}\right\}_{w \in \mathrm{I}_{\mathcal{K}}}$ ) is called the standard $p$-adic (resp. complex) basis of $V \otimes_{\mathbf{Q}} \mathbf{Q}_{p}$ (resp. $\left.V \otimes_{\mathbf{Q}} \mathbf{C}\right)$. Weidentify $\mathrm{GL}\left(V_{\Sigma}\right)\left(\right.$ resp. $\left.\mathrm{GL}\left(V_{\Sigma^{c}}\right)\right)$ with $\mathrm{GL}_{r+s}\left(\mathcal{F}_{p}\right)=\prod_{v \in S_{p}} \operatorname{GL}_{r+s}\left(\mathcal{F}_{v}\right)$ with respect to the standard $p$-adic basis of $V_{\Sigma}$ (resp. $V_{\Sigma^{c}}$ ). Consider the following embedding:

$$
\begin{aligned}
\mathrm{R}_{\mathcal{F} / \mathbf{Q}} G\left(\mathbf{Q}_{p}\right) & =\prod_{v \in S_{p}} G\left(\mathcal{F}_{v}\right) \rightarrow \mathrm{GL}\left(V_{\Sigma}\right) \times \mathrm{GL}\left(V_{\Sigma^{c}}\right) \times \mathcal{F}_{p}^{\times} \\
& =\prod_{v \in S_{p}} \mathrm{GL}_{r+s}\left(\mathcal{F}_{v}\right) \times \mathrm{GL}_{r+s}\left(\mathcal{F}_{v}\right) \times \mathcal{F}_{v}^{\times} \\
g & \mapsto\left(\left.g\right|_{V_{\Sigma}},\left.g\right|_{V_{\Sigma^{c}}}, \nu(g)\right) .
\end{aligned}
$$

For each $v \in S_{p}$, the above embedding gives rises to an identification

$$
\begin{aligned}
& G U(V)\left(\mathcal{F}_{v}\right) \\
& \quad=G\left(\mathcal{F}_{v}\right) \simeq\left\{\left(x, x^{\prime}, \nu\right) \in \mathrm{GL}_{r+s}\left(\mathcal{F}_{v}\right) \times \mathrm{GL}_{r+s}\left(\mathcal{F}_{v}\right) \times \mathcal{F}_{v}^{\times} \mid x^{\prime}=\vartheta_{r, s}^{t} x^{-t} \vartheta_{r, s}^{-t} \cdot \nu\right\} .
\end{aligned}
$$

If $w$ is the place above $p$ in $\Sigma_{p}$, then we have the identifications:

$$
\begin{aligned}
G U\left(\mathcal{F}_{v}\right) & =\mathrm{GL}_{r+s}\left(\mathcal{F}_{v}\right) \times \mathcal{F}_{v}^{\times}, \quad g \rightarrow\left(\left.g\right|_{e_{w} V}, \nu(g)\right), \\
U(V)\left(\mathcal{F}_{v}\right) & =\mathrm{GL}_{r+s}\left(\mathcal{F}_{v}\right),\left.\quad g \rightarrow g\right|_{e_{w} V} .
\end{aligned}
$$

Thus, $(g, \nu) \in G U\left(\mathcal{F}_{v}\right)$ has the similitude $\nu$. Similarly, the standard complex basis gives rise to the identification:

$$
\mathrm{R}_{\mathcal{F} / \mathbf{Q}} G(\mathbf{R})=\mathrm{GL}_{r+s}(\mathbf{C}(\Sigma)) \times \mathbf{C}^{\times}(\Sigma) .
$$

We shall fix these identifications throughout this article.

1.8. Lattices and polarization. In what follows we make a choice of the lattice $M$ in $V$. Denote by $\mathfrak{d}_{\mathcal{K}}$ the absolute different of $\mathcal{K} / \mathbf{Q}$. Let $X^{\vee}=\mathfrak{d}_{\mathcal{K}}^{-1} x^{1} \oplus \cdots \mathfrak{d}_{\mathcal{K}}^{-1} x^{s}=$ $\left(\mathfrak{d}_{\mathcal{K}}^{-1}\right)^{s}$ and $Y=\mathcal{O}_{\mathcal{K}} y^{1} \oplus \cdots \mathcal{O}_{\mathcal{K}} y^{s}=\mathcal{O}_{\mathcal{K}}^{s}$ be the standard $\mathcal{O}_{\mathcal{K}}$-lattices in $X_{\mathcal{K}}$ and $Y_{\mathcal{K}}$ respectively. We choose an $\mathcal{O}_{\mathcal{K}}$-lattice $L$ in $W$ such that $\operatorname{Tr}_{\mathcal{K} / \mathbf{Q}}(\vartheta(L, L)) \subset \mathbf{Z}$ and $L_{p}=L \otimes_{\mathbf{Z}} \mathbf{Z}_{p}=\sum_{i=1}^{r-s}\left(\mathcal{O}_{\mathcal{K}} \otimes_{\mathbf{Z}} \mathbf{Z}_{p}\right) w^{i}=\left(\mathcal{O}_{\mathcal{K}} \otimes_{\mathbf{Z}} \mathbf{Z}_{p}\right)^{r-s}$. Define the $\mathcal{O}_{\mathcal{K}}$-lattice $M$ in $V$ by

$$
M:=Y \oplus L \oplus X^{\vee} .
$$

Then $\langle M, M\rangle_{r, s} \subset \mathbf{Z}$ and $M_{p}:=M \otimes_{\mathbf{Z}} \mathbf{Z}_{p}$ is self-dual with respect to $\langle,\rangle_{r, s}$ as $p$ is unramified in $\mathcal{K}$. A pair of sublattices $\operatorname{Pol}_{p}=\left\{N^{-1}, N^{0}\right\}$ of $M_{p}$ is called an ordered polarization of $M_{p}$ if $N^{-1}$ and $N^{0}$ are maximal isotropic submodules in $M_{p}$ and they are dual to each other with respect to $\langle,\rangle_{r, s}$. Moreover, we require that

$$
\operatorname{rank} N_{\Sigma}^{-1}=\operatorname{rank} N_{\Sigma^{c}}^{0}=r, \operatorname{rank} N_{\Sigma^{c}}^{-1}=\operatorname{rank} N_{\Sigma}^{0}=s
$$

We now endow $M$ with the standard ordered polarization as follows. Put

$$
M^{-1}=Y_{\Sigma} \oplus L_{\Sigma} \oplus Y_{\Sigma^{c}} \text { and } M^{0}=X_{\Sigma^{c}}^{\vee} \oplus L_{\Sigma^{c}} \oplus X_{\Sigma}^{\vee}
$$


M.-L. HSIEH

We call $\operatorname{Pol}_{p}^{0}=\left\{M^{-1}, M^{0}\right\}$ the standard (ordered) polarization of $M_{p}$. We make the following identification according to the standard $p$-adic basis.

$$
\begin{aligned}
& M_{\Sigma}^{0}=X_{\Sigma}^{\vee}=O_{p}(\Sigma)^{s} \quad \text { and } \quad M_{\Sigma^{c}}^{-1}=Y_{\Sigma^{c}}=O_{p}\left(\Sigma^{c}\right)^{s} \\
& M_{\Sigma}^{-1}=Y_{\Sigma} \oplus L_{\Sigma}=O_{p}(\Sigma)^{r} \quad \text { and } \quad M_{\Sigma^{c}}^{0}=X_{\Sigma^{c}}^{\vee} \oplus L_{\Sigma^{c}}=O_{p}\left(\Sigma^{c}\right)^{r} .
\end{aligned}
$$

1.9. Filtration. Let $R$ be a ring and $N$ be a free $R$-module of rank $l$ with an ordered basis $\left\{v^{1}, \ldots, v^{l}\right\}$, and define the standard (decreasing) filtration Fil ${ }_{s t}^{\bullet}(N$, $\left.\left.\left\{v^{1}, \ldots, v^{l}\right\}\right)\right)$ of $N$ by $\operatorname{Fil}_{s t}^{n}\left(N,\left\{v^{1}, \ldots, v^{l}\right\}\right)=\sum_{i=1}^{l-n+1} R v^{i}$. We endow the $O_{p^{-}}$ modules $M_{\Sigma}^{0}$ and $M_{\Sigma^{c}}^{0}$ with the filtration defined by

$$
\begin{gathered}
\operatorname{Fil}^{i} M_{\Sigma}^{0}=\operatorname{Fil}_{s t}^{i}\left(X_{\Sigma}^{\vee},\left\{e^{+} x^{s}, \ldots, e^{+} x^{1}\right\}\right) \\
\operatorname{Fil}^{i} M_{\Sigma^{c}}^{0}= \begin{cases}\operatorname{Fil}_{s t}^{i-s}\left(L_{\Sigma^{c}},\left\{e^{-} w^{1}, \ldots, e^{-} w^{r-s}\right\}\right) & \text { if } 1 \leq i \leq s, \\
L_{\Sigma^{c}} \oplus \operatorname{Fil}_{s t}^{i}\left(X_{\Sigma^{c}}^{\vee},\left\{e^{-} x^{1}, \ldots, e^{-} x^{r}\right\}\right) & \text { if } 1 \leq i \leq s .\end{cases}
\end{gathered}
$$

Define the filtration Fil ${ }^{\bullet} M_{\Sigma}$ of $M_{\Sigma}$ as follows:

$$
\text { Fil }^{i} M_{\Sigma}= \begin{cases}\operatorname{Fil}_{s t}^{i-r}\left(X_{\Sigma}^{\vee},\left\{e^{+} x^{s}, \ldots, e^{+} x^{1}\right\}\right) & \text { if } r+1 \leq i \leq r+s, \\ \operatorname{Fil}_{s t}^{i-s}\left(L_{\Sigma},\left\{e^{+} w^{r-s}, \ldots, e^{+} w^{1}\right\}\right) \oplus M_{\Sigma}^{0} & \text { if } s+1 \leq i \leq r, \\ \operatorname{Fil}_{s t}^{i}\left(Y_{\Sigma},\left\{e^{+} y^{s}, \ldots, e^{+} y^{1}\right\}\right) \oplus L_{\Sigma} \oplus M_{\Sigma}^{0} & \text { if } 1 \leq i \leq s .\end{cases}
$$

1.10. Open-compact subgroups. For $v \in \mathbf{h}$, put

$$
K_{v}^{0}:=\left\{g \in G\left(\mathcal{F}_{v}\right) \mid\left(M \otimes_{O} O_{v}\right) \cdot g=M \otimes_{O} O_{v}\right\}
$$

and $K^{0}=\prod_{v \in \mathbf{h}} K_{v}^{0}$. Let $N_{0} \geq 3$ be a prime-to- $p$ positive integer and fix an opencompact subgroup $K$ such that $K_{v}=K_{v}^{0}$ for all $v \mid p$. We assume $K$ is neat in the following sense:

(neat)

$$
\begin{aligned}
& K \subset\left\{g \in G\left(\mathbf{A}_{\mathcal{F}, f}\right) \mid M(g-1) \subset N_{0} \cdot M\right\} \\
& \nu(K) \cap O_{+}^{\times} \subset\left(K \cap O^{\times}\right)^{2} .
\end{aligned}
$$

The first condition assures that $K$ is torsion free ([Shi97, Lemma 24.3 (2)]), and the second one is possible by choosing sufficiently large $N_{0}$ ( $c f$. Hid04b, page 136]). Under the identification (1.2), we have

$$
K_{v}^{0}=\mathrm{GL}\left(M_{\Sigma}\right) \times O_{v}^{\times} \stackrel{\sim}{\rightarrow} \mathrm{GL}_{r+s}\left(O_{v}\right) \times O_{v}^{\times} \text {for } v \mid p .
$$

For $g_{p}=\left(g_{p}^{(1)}, \nu\left(g_{p}\right)\right) \in K_{p}^{0}$, we write $g_{p}^{(1)}=\left[\begin{array}{ll}A & B \\ C & D\end{array}\right]$ according to the decomposition $M_{\Sigma}=M_{\Sigma}^{-1} \oplus M_{\Sigma}^{0}$. For $n \in \mathbf{Z}_{+}$, put

$$
I_{1}\left(p^{n}\right)=\left\{g \in K_{p}^{0} \mid g_{p} \in N_{r+s}\left(O_{p}\right) \times\{1\}\left(\bmod p^{n}\right)\right\}
$$

and define

$$
\begin{aligned}
K^{n} & =\left\{g \in K \mid g_{p} \equiv\left[\begin{array}{cc}
\mathbf{1}_{r} & * \\
0 & \mathbf{1}_{s}
\end{array}\right] \times\{1\} \bmod p^{n}\right\}, \\
K_{1}^{n} & =\left\{g \in K\left|g_{p}^{(1)}\right|_{\mathrm{gr} \bullet} M_{\Sigma} \equiv 1\left(\bmod p^{n}\right), \nu\left(g_{p}\right) \equiv 1\left(\bmod p^{n}\right)\right\}=K^{(p)} I_{1}\left(p^{n}\right), \\
K_{0}^{n} & =\left\{g \in K \mid g_{p}^{(1)} \in B_{r+s}\left(O_{p}\right)\left(\bmod p^{n}\right)\right\} .
\end{aligned}
$$




\section{SHIMURA VARIETIES FOR UNITARY GROUPS}

One approach to explore the arithmetic of modular forms on unitary groups is to study the associated Shimura varieties and understand their structure as a moduli space of certain abelian schemes with additional structures. We review these objects in this section, following the exposition in [Hid04b, Chapter 7]. In what follows, let $\mathcal{K}$ be a finite extension of $\mathcal{K}^{n c}\left(e^{2 \pi i / N_{0}}\right)$ which is unramified at $p$ and let $\mathfrak{p}$ be the prime ideal of $\mathcal{K}$ induced by $\iota_{p}: \mathcal{K} \hookrightarrow \mathbf{C}_{p}$. Let $\mathcal{O}:=\mathcal{O}_{\mathcal{K},(\mathfrak{p})}$ be the localization of $\mathcal{O}_{\mathcal{K}}$ at $\mathfrak{p}$ and let $\mathcal{O}_{\mathfrak{p}}$ be the $\mathfrak{p}$-adic completion of $\mathcal{O}$. We shall identify $\mathcal{O}_{\mathfrak{p}}$ with the $p$-adic closure of $\iota_{p}(\mathcal{O})$ in $\mathbf{C}_{p}$ via $\iota_{p}$. An $\mathcal{O}$-algebra $R$ is called a base ring, and similarly a scheme $S$ over Spec $\mathcal{O}$ is called a base scheme.

2.1. Shimura varieties associated to $\boldsymbol{G} \boldsymbol{U}(\boldsymbol{V})$. Let $\square$ be a finite set of rational primes not dividing $N_{0}$. Let $U \subset K$ be an open-compact subgroup in $\mathrm{R}_{\mathcal{F} / \mathbf{Q}} G\left(\mathbf{A}_{\mathbf{Q}, f}\right)$.

Definition 2.1 ( $S$-quadruple). Let $S$ be a locally noetherian connected $\mathcal{O}$-scheme and let $\bar{s}$ be a geometric point of $S$. An $S$-quadruple of level $U^{(\square)}$ is a quadruple $\underline{A}=\left(A, \bar{\lambda}, \iota, \bar{\eta}^{(\square)}\right)_{S}$ consisting of the following data:

- $A$ is an abelian scheme of dimension $(r+s) d$ over $S$.

- $\iota: \mathcal{O}_{\mathcal{K}} \hookrightarrow \operatorname{End}_{S} A \otimes \mathbf{z} \mathbf{Z}_{(\square)}$.

- $\lambda$ is a prime-to- $\square$ polarization of $A$ over $S$ and $\bar{\lambda}$ is the $O_{(\square),+}$-orbit of $\lambda$. Namely,

$\bar{\lambda}=O_{(\square),+} \lambda:=\left\{\lambda^{\prime} \in \operatorname{Hom}\left(A, A^{t}\right) \otimes_{\mathbf{z}} \mathbf{Z}_{(\square)} \mid \lambda^{\prime}=\lambda \circ a, a \in O_{(\square),+}\right\}$.

- $\bar{\eta}^{(\square)}=\eta^{(\square)} U$ is a $\pi_{1}(S, \bar{s})$-invariant $U$-orbit of the isomorphism of $\mathcal{O}_{\mathcal{K}^{-}}$ modules $\eta^{(\square)}: M \otimes \widehat{\mathbf{Z}}^{(\square)} \stackrel{\sim}{\rightarrow} T^{(\square)}\left(A_{\bar{s}}\right):=H_{1}\left(A_{\bar{s}}, \widehat{\mathbf{Z}}^{(\square)}\right)$. Here we define $\eta^{(\square)} g(x)=\eta\left(x g^{\vee}\right)$ for $g \in U$.

Furthermore, the quadruple $\left(A, \bar{\lambda}, \iota, U \eta^{(\square)}\right)_{S}$ satisfies the following conditions (K1)-(K3):

(K1) Let ${ }^{t}$ denote the Rosati involution induced by $\lambda$ on $\operatorname{End}_{S} A \otimes \mathbf{Z}_{(\square)}$. Then $\iota(b)^{t}=\iota(c(b)), \forall b \in \mathcal{O}_{\mathcal{K}}$.

(K2) Let $e^{\lambda}$ be the Weil pairing induced $\lambda$. Lifting the isomorphism $\mathbf{Z} / N_{0} \mathbf{Z} \simeq$ $\mathbf{Z} / N_{0} \mathbf{Z}(1)$ induced by $e^{2 \pi i / N_{0}}$ to an isomorphism $\zeta: \hat{\mathbf{Z}} \simeq \hat{\mathbf{Z}}(1)$, we can regard $e^{\lambda}$ as a skew-Hermitian form $e^{\lambda}: T^{(\square)}\left(A_{\bar{s}}\right) \times T^{(\square)}\left(A_{\bar{s}}\right) \rightarrow \mathfrak{d}_{\mathcal{K}}^{-1} \otimes_{\mathbf{Z}} \widehat{\mathbf{Z}}^{(\square)}$. Let $e^{\eta}$ denote the skew-Hermitian form on $T^{(\square)}(A)$ induced by $e^{\eta}\left(x, x^{\prime}\right)=$ $\vartheta_{r, s}\left(\eta(x), \eta\left(x^{\prime}\right)\right)$. We require that

$$
e^{\lambda}=u \cdot e^{\eta} \text { for some } u \in \mathbf{A}_{\mathcal{F}, f}^{(\square)} \text {. }
$$

(K3) The determinant condition:

$$
\operatorname{det}(X-\iota(b) \mid \operatorname{Lie} A)=\prod_{\sigma \in \Sigma}(X-(\sigma c)(b))^{r}(X-\sigma(b))^{s} \in \mathcal{O}_{S}[X], \forall b \in \mathcal{O}_{\mathcal{K}}
$$

Define the fibred category $\mathfrak{C}_{U}^{(\square)}$ over $S C H_{\mathcal{O}_{\mathcal{K},(\square)}}$ as follows. Objects over $S$ are $S$-quadruples. For $\underline{A}=\left(A, \bar{\lambda}, \iota, \bar{\eta}^{(\square)}\right)_{S}$ and $\underline{A^{\prime}}=\left(A^{\prime}, \bar{\lambda}^{\prime}, \iota^{\prime}, \bar{\eta}^{\prime(\square)}\right)_{S}$, we define the morphism by

$$
\operatorname{Hom}_{\mathfrak{C}_{U}^{(\square)}}\left(\underline{A}, \underline{A^{\prime}}\right)=\left\{\phi \in \operatorname{Hom}_{\mathcal{O}_{\mathcal{K}}}\left(A, A^{\prime}\right) \mid \phi^{*} \bar{\lambda}^{\prime}=\bar{\lambda}, \phi\left(\bar{\eta}^{(\square)}\right)=\bar{\eta}^{(\square)}\right\} .
$$

We say $\underline{A} \simeq \underline{A}^{\prime}$ if there exists an isomorphism in $\operatorname{Hom}_{\mathfrak{C}_{U}^{(\square)}}\left(\underline{A}, \underline{A^{\prime}}\right)$. 
If $\square=\emptyset$ is the empty set, we define the functor $\mathfrak{S}_{U}: S C H_{/ \mathcal{K}} \rightarrow S E T S$ by

$$
\mathfrak{S}_{U}(S)=\left\{\underline{A}=(A, \bar{\lambda}, \iota, \eta U)_{S} \in \mathfrak{C}_{U}(S)\right\} / \simeq .
$$

By the theory of Shimura-Deligne, $\mathfrak{S}_{U}$ is represented by a quasi-projective scheme $S_{G}(U)_{/ \mathcal{K}}$ over $\mathcal{K}$. We call $S_{G}(U)_{/ \mathcal{K}}$ the Shimura variety attached to $G=G U(V)$ over $\mathcal{K}$.

2.2. Kottwitz model. Suppose that $\square=\{p\}$. Let $K$ be an open-compact subgroup such that $K_{p}=K_{p}^{0}$. Define the functor $\mathfrak{S}_{K}^{(p)}: S C H_{/ \mathcal{O}} \rightarrow S E T S$ by

$$
\mathfrak{S}_{K}^{(p)}(S)=\left\{\underline{A}=\left(A, \bar{\lambda}, \iota, \bar{\eta}^{(p)}\right)_{S} \in \mathfrak{C}_{K}^{(p)}(S)\right\} / \simeq .
$$

In Kot92, Kottwitz shows that $\mathfrak{S}_{K}^{(p)}$ is represented by a quasi-projective scheme $S_{G}(K)_{/ \mathcal{O}}$ over $\mathcal{O}$ if $K$ is neat.

2.3. Igusa schemes associated to $\boldsymbol{G} \boldsymbol{U}(\boldsymbol{V})$. Let $\operatorname{Pol}_{p}=\left\{N^{-1}, N^{0}\right\}$ be a polarization of $M_{p}=M \otimes_{\mathbf{z}} \mathbf{Z}_{p}$, where $M$ is the $\mathcal{O}_{\mathcal{K}}$-lattice defined in (1.3).

Definition 2.2 ( $S$-quintuples). Let $n$ be a positive integer. Define the fibred category $\mathfrak{C}_{K, n, \text { Pol }_{p}}^{(p)}$ whose objects over a base scheme $S$ are $S$-quintuples $(\underline{A}, j)_{S}=$ $\left(A, \bar{\lambda}, \iota, \bar{\eta}^{(p)}, j\right)_{S}$ of level $K^{n}$, where $\underline{A} \in \mathfrak{C}_{K}^{(p)}(S)$ is a $S$-quadruple and

$$
j: \boldsymbol{\mu}_{p^{n}} \otimes \mathbf{z} N^{0} \hookrightarrow A\left[p^{n}\right]
$$

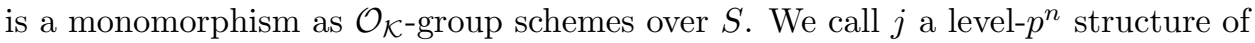
$A$. Morphisms between $S$-quintuples are

$$
\operatorname{Hom}_{\mathfrak{C}_{K, n, \mathrm{Pol}_{p}}^{(p)}}\left((\underline{A}, j),\left(\underline{A^{\prime}}, j^{\prime}\right)\right)=\left\{\phi \in \operatorname{Hom}_{\mathfrak{C}_{K}^{(p)}}\left(\underline{A}, \underline{A^{\prime}}\right) \mid \phi j=j^{\prime}\right\} .
$$

Define the functor $\mathfrak{I}_{K, n, \mathrm{Pol}_{p}}^{(p)}: S C H_{/ \mathcal{O}} \rightarrow S E T S$ by

$$
\mathfrak{I}_{K, n, \operatorname{Pol}_{p}}^{(p)}(S)=\left\{(\underline{A}, j)=\left(A, \bar{\lambda}, \iota, \bar{\eta}^{(p)}, j\right)_{S} \in \mathfrak{C}_{K, n}^{(p)}(S)\right\} / \simeq .
$$

It is known that $\mathfrak{I}_{K, n, \mathrm{Pol}_{p}}^{(p)}$ are relatively representable over $S_{G}(K)_{/ \mathcal{O}}(c f$. HLS06, Lemma(2.1.6.4)] and [SGA64, Prop. 3.12]), and thus it is represented by a scheme. Denote by $I_{G}^{0}\left(K^{n}\right) / \mathcal{O}$ the scheme that represents $\mathfrak{I}_{K, n, \mathrm{Pol}_{p}^{0}}^{(p)}$ for the standard polarization $\operatorname{Pol}_{p}^{0}=\left\{M^{-1}, M^{0}\right\}$ defined in (1.4). Let $\underline{\mathcal{A}}$ be the universal quadruple of level $K^{(p)}$ over $S_{G}(K)$. Then we have

$$
\underline{I_{G}^{0}}\left(K^{n}\right)=\underline{\operatorname{Inj}}_{\mathcal{O}_{\mathcal{K}}}\left(\boldsymbol{\mu}_{p^{n}} \otimes_{\mathbf{Z}} M^{0}, \mathcal{A}\right)
$$

In addition, $I_{G}^{0}\left(K^{n}\right)$ is a model of $S_{G}\left(K^{n}\right)$ over $\mathcal{O}$ in view of the following lemma.

Lemma 2.3. Let $L \supset \mathcal{K}\left(e^{2 \pi i / p^{n}}\right)$ be a field. There is a non-canonical isomorphism over $\operatorname{Spec} L$.

$$
I_{G}^{0}\left(K^{n}\right) / L \stackrel{\sim}{\rightarrow} S_{G}\left(K^{n}\right)_{/ L}
$$

Proof. Since $M_{\Sigma}=M_{\Sigma}^{0} \oplus M_{\Sigma}^{-1}$, we have a natural exact sequence

$$
0 \longrightarrow M_{\Sigma}^{0} \stackrel{i^{0}}{\longrightarrow} M_{\Sigma} \stackrel{i^{-1}}{\longrightarrow} M_{\Sigma}^{-1} \longrightarrow 0 .
$$

We fix an isomorphism $\zeta_{p^{n}}: \mathbf{Z}_{n} \simeq \boldsymbol{\mu}_{p^{n}}$, which induces an isomorphism $\zeta_{p^{n}}^{-1}$ : $\boldsymbol{\mu}_{p^{n}} \otimes M_{\Sigma}^{0} \simeq \mathbf{Z}_{n} \otimes M_{\Sigma}^{0}$. Let $S$ be a scheme over $L$ and $\underline{A}=\left(A, \bar{\lambda}, \eta^{(p)} K\right)_{S}$ be 
an $S$-quadruple of level $K^{(p)}$. A level- $p^{n}$ structure $j$ of $\underline{A}$ is equivalent to a class $\eta_{p}(j) K\left(p^{n}\right)$, where $\eta_{p}(j): M^{-1} \oplus M^{0}=M_{p} \stackrel{\sim}{\rightarrow} T_{p}(A)$. We can define the isomorphism (dependent on the choice of $\left.\zeta_{p^{n}}\right) I_{G}^{0}\left(K^{n}\right)_{/ L} \stackrel{\sim}{\rightarrow} S_{G}\left(K^{n}\right)_{/ L}$ by

$$
\left[\left(A, \bar{\lambda}, \iota, \bar{\eta}^{(p)}, j\right)\right] \rightarrow\left[\left(A, \bar{\lambda}, \iota,\left(\eta^{(p)} \times \eta_{p}(j)\right) K^{n}\right)\right] .
$$

2.3.1. Change of the polarization. It is clear that the notion of level- $p^{n}$ structures depends on the choice of the polarization of $M_{p}$. Choose $\gamma \in K_{p}^{0}$ such that $N^{-1}=$ $M^{-1} \gamma$ and $N^{0}=M^{0} \gamma$. Then we see that $j \mapsto \gamma j$ is an isomorphism from the level$p^{n}$ structures with respect to $\operatorname{Pol}_{p}^{0}$ to those of $\operatorname{Pol}_{p}$. Therefore the map $[(\underline{A}, j)] \rightarrow$ $[(\underline{A}, \gamma j)]$ induces an isomorphism between $\mathfrak{I}_{K, n, \mathrm{Pol}_{p}^{0}}^{(p)}$ and $\mathfrak{I}_{K, n, \mathrm{Pol}_{p}}^{(p)}$.

2.3.2. $p$-adic one forms. Suppose $p$ is nilpotent in $R$ and $p^{m} R=0$ for some $m \geq 1$. Let $(\underline{A}, j)$ be an $R$-quintuple of level $K^{n}, n \geq m$. Identify $M^{0}=M_{\Sigma}^{-1} \oplus M_{\Sigma^{c}}^{0}$ with the basis in (1.4). Then the level $p^{n}$-structure $j$ over $R$ induces a trivialization of Lie $A$ :

$j_{*}^{+}: M_{\Sigma}^{0} \otimes R \stackrel{\sim}{\rightarrow} e^{+} \operatorname{Lie} A\left[p^{n}\right]=e^{+} \operatorname{Lie} A ; j_{*}^{-}: M_{\Sigma^{c}}^{0} \otimes R \stackrel{\sim}{\rightarrow} e^{-} \operatorname{Lie} A\left[p^{n}\right]=e^{-} \operatorname{Lie} A$.

Let $\underline{\omega}_{A}=\operatorname{Hom}(\operatorname{Lie} A, R)$ be the $R$-module of invariant one forms of $A$. Taking the duality $\operatorname{Hom}_{O_{p}}\left(-, O_{p}\right)$ of the identification in (1.4), we obtain an isomorphism induced by $j_{*}$ :

$$
\boldsymbol{\omega}(j)^{+}=\boldsymbol{\omega}\left(j^{-}\right): O_{p}(\Sigma)^{r} \otimes R \stackrel{\sim}{\rightarrow} e^{+} \underline{\omega}_{A} ; \boldsymbol{\omega}(j)^{-}=\boldsymbol{\omega}\left(j^{+}\right): O_{p}\left(\Sigma^{c}\right)^{s} \otimes R \stackrel{\sim}{\rightarrow} e^{-} \underline{\omega}_{A} .
$$

2.4. Complex uniformization. Let $U \subset K^{0}$ be an open compact subgroup in $G\left(\mathbf{A}_{\mathcal{F}, f}\right)$. We recall the description of the complex points $S_{G}(U)(\mathbf{C})$ following [Shi98, Chap. VI].

2.4.1. We begin with the Hermitian symmetric domain attached to unitary groups with signature $(r, s)$. We treat two cases $r \geq s>0$ and $r s=0$ separately. If $r \geq s>0$, we put

$$
X_{r, s}=\left\{\tau=\left[\begin{array}{l}
x \\
y
\end{array}\right] \mid x \in M_{s}\left(\mathbf{C}^{\Sigma}\right), y \in M_{(r-s) \times s}\left(\mathbf{C}^{\Sigma}\right), i\left(x^{*}-x\right)>-i y^{*} \vartheta^{-1} y\right\} .
$$

According to the standard basis of $V$, we can write $\alpha \in \mathrm{R}_{\mathcal{F} / \mathbf{Q}} G(\mathbf{R})$ in the form

$$
\alpha=\left[\begin{array}{lll}
a & b & c \\
g & e & f \\
h & l & d
\end{array}\right]
$$

with $a, d \in M_{r}\left(\mathbf{C}^{\Sigma}\right)$ and $e \in M_{r-s}\left(\mathbf{C}^{\Sigma}\right)$. The action of $\alpha \in \mathrm{R}_{\mathcal{F} / \mathbf{Q}} G(\mathbf{R})^{+}$on $X_{r, s}$ is defined by

$$
\alpha\left[\begin{array}{l}
x \\
y
\end{array}\right]=\left[\begin{array}{l}
a x+b y+c \\
g x+e y+f
\end{array}\right] \cdot(h x+l y+d)^{-1} .
$$

If $r s=0$, then $X_{r, s}$ consists of a single point written $\mathbf{x}_{0}$ with the trivial action of $G$.

Then $X^{+}:=X_{r, s}$ is the Hermitian symmetric domain associated to $G$. Put

$$
M_{G}\left(X^{+}, U\right):=G(\mathcal{F})^{+} \backslash X^{+} \times G\left(\mathbf{A}_{\mathcal{F}, f}\right) / U,
$$

where $G(\mathcal{F})^{+}=\{g \in G(\mathcal{F}) \mid \nu(g)>0\}$. Then $M_{G}\left(X^{+}, U\right)$ is a complex manifold when $U$ is neat. Denote by $[\tau, g] \in M_{G}\left(X^{+}, U\right)$ the complex point represented 
M.-L. HSIEH

by $(\tau, g) \in X^{+} \times G\left(\mathbf{A}_{\mathcal{F}, f}\right)$. The group $G=G U(r, s)$ satisfies the Hasse principle ([Hid04b, 7.1.5, page 319]). Hence we have

$$
M_{G}\left(X^{+}, U\right) \stackrel{\sim}{\rightarrow} S_{G}(U)(\mathbf{C}) .
$$

2.4.2. Analytic construction of the universal abelian scheme over $\mathbf{C}$. Let $\underline{\mathcal{A}}(V) / \mathbf{C}$ be the universal quadruple of level $U$ over $S_{G}(U)_{\mathbf{C}}$. We shall recall a construction of $\underline{\mathcal{A}}(V)_{\mathbf{C}}$ after introducing some notation. Define the $\mathcal{K} \otimes_{\mathbf{Q}} \mathbf{C}$-module $\mathbf{C}^{r, s}$ by

$$
\mathbf{C}^{r, s}=\mathbf{C}\left(\Sigma^{c}\right)^{s} \oplus \mathbf{C}\left(\Sigma^{c}\right)^{r-s} \oplus \mathbf{C}(\Sigma)^{s} .
$$

Here $\mathbf{C}^{r-s}$ and $\mathbf{C}^{s}$ are regarded as spaces of row vectors. According to the above decomposition (2.6), we define $c_{r, s}: \mathbf{C}^{r, s} \rightarrow \mathbf{C}^{r, s}$ by

$$
\left(u_{1}, u_{2}, u_{3}\right) c_{r, s}=\left(\overline{u_{1}}, \overline{u_{2}}, u_{3}\right),
$$

where $\bar{u}$ means the complex conjugation of $u \in \mathbf{C}$. We denote by $z_{V}^{(1)}(\Sigma), \ldots, z_{V}^{(r)}(\Sigma)$ the first $r d$-tuple complex coordinates of $\mathbf{C}^{r, s}$, where $z_{V}^{(i)}(\Sigma)=\left(z_{V, \sigma}^{(i)}\right)_{\sigma \in \Sigma}$. Similarly, we denote by $z_{V}^{(r+1)}\left(\Sigma^{c}\right), \ldots, z_{V}^{(r+s)}\left(\Sigma^{c}\right)$ the rest of $s d$-tuple complex coordinates. If $s>0$, we put

$$
B(\tau)=\left[\begin{array}{ccc}
x^{*} & y^{*} & x \\
0 & \vartheta & y \\
\mathbf{1}_{s} & 0 & \mathbf{1}_{s}
\end{array}\right] \in M_{r+s}\left(\mathbf{C}^{\Sigma}\right) .
$$

If $r s=0$, we put $B\left(\mathbf{x}_{0}\right)=\vartheta$. The CM-type $\Sigma$ induces an isomorphism $\Sigma$ : $\mathcal{K} \otimes_{\mathbf{Q}} \mathbf{R} \stackrel{\sim}{\rightarrow} \mathbf{C}^{\Sigma}$, by which we regard $V \otimes_{\mathbf{Q}} \mathbf{R}$ as a $\mathbf{C}$-space of row vectors according to the $\mathcal{K}$-basis $\left\{y^{i}, w^{i}, x^{i}\right\}$. For each $\tau \in X^{+}$, we define the map $p(\tau): V \otimes_{\mathbf{Q}} \mathbf{R} \stackrel{\sim}{\rightarrow} \mathbf{C}^{r, s}$ by $p(\tau) v=v B(\tau) c_{r, s}$. Then $V$ acts on $(\tau, z) \in X^{+} \times \mathbf{C}^{r, s}$ by

$$
v \cdot(\tau, z)=(\tau, p(\tau) v+z) .
$$

We define a left action of $G$ on $V$ by

$$
g * v:=v g^{\vee}=v g^{-1} \nu(g) .
$$

Put

$$
M_{[g]}=g * M=M g^{\vee} \text { and } M_{[g]}(\tau)=p(\tau)\left(M_{[g]}\right) .
$$

To each point $(\tau, g) \in X^{+} \times G\left(\mathbf{A}_{\mathcal{F}, f}\right)$ we can attach $\underline{\mathcal{A}}(V)_{g}(\tau)=$

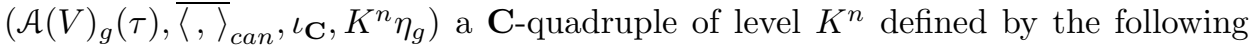
data.

- The abelian variety: $\mathcal{A}(V)_{g}(\tau):=\mathbf{C}^{r, s} / M_{[g]}(\tau)$ with $p(\tau): V \otimes_{\mathbf{Q}} \mathbf{R} / M_{[g]} \stackrel{\sim}{\rightarrow}$ $\mathcal{A}_{g}(\tau)$

- The polarization: $\overline{\langle,\rangle}_{c a n}$ is the $\mathcal{F}_{+}$-orbit of the polarization induced by the unique Riemann form $\langle,\rangle_{c a n}$ on $\mathbf{C}^{r, s}$ such that $\left\langle p(\tau) v, p(\tau) v^{\prime}\right\rangle_{c a n}=$ $\left\langle v, v^{\prime}\right\rangle_{r, s}$ for all $v, v^{\prime} \in V$.

- The endomorphism: $\iota_{\mathbf{C}}: \mathcal{O}_{\mathcal{K}} \rightarrow$ End $\mathcal{A}_{g}(\tau) \otimes_{\mathbf{Z}} \mathbf{Q}$ is the $\mathcal{O}_{\mathcal{K}}$-action induced by the action on $V$ via $p(\tau)$,

- The prime-to- $p$ level structure: $\eta_{g}^{(p)}: M \otimes \hat{\mathbf{Z}}^{p} \stackrel{\sim}{\rightarrow} M_{[g]}=H_{1}\left(\mathcal{A}_{g}(\tau), \hat{\mathbf{Z}}^{p}\right)$ is defined by

$$
\eta_{g}^{(p)}(x)=g * x \text { for } x \in M \text {. }
$$


- The level structure at $p$ : fix a primitive $p^{n}$-th $\operatorname{root} \zeta=e^{2 \pi i / p^{n}}$ and let $\zeta: \mathbf{Z} / p^{n} \mathbf{Z} \stackrel{\sim}{\rightarrow} \boldsymbol{\mu}_{p^{n}}$ be the induced isomorphism. Define

$$
j_{\zeta}: M^{0} \otimes \boldsymbol{\mu}_{p^{n}} \simeq M^{0} \otimes \mathbf{Z} / p^{n} \mathbf{Z} \hookrightarrow \mathcal{A}_{g}(\tau)\left[p^{n}\right]=M_{[g]} \otimes \mathbf{Z} / p^{n} \mathbf{Z}
$$

by $j_{\zeta}\left(x^{0}\right)=g * x^{0}$ for $x^{0} \in M^{0}$.

Let $\eta_{g}$ be the full level structure $\eta_{g}^{(p)} \times \eta_{p}\left(j_{\zeta}\right)$. The isomorphism in (2.5) can be described explicitly as follows:

$$
\begin{aligned}
M_{G}\left(X^{+}, K^{n}\right) & \stackrel{\sim}{\rightarrow} S_{G}\left(K^{n}\right)(\mathbf{C}) \\
{[\tau, g] } & \rightarrow \underline{\mathcal{A}}(V)_{g}(\tau):=\left[\left(\mathcal{A}(V)_{g}(\tau), \overline{\langle,\rangle}_{c a n}, \iota_{\mathbf{C}}, \eta_{g} K^{n}\right)\right] .
\end{aligned}
$$

By definition, we have

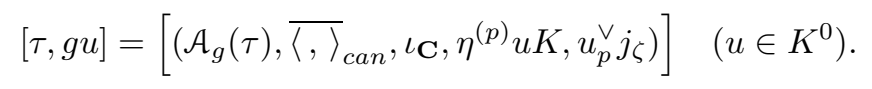

We put

$$
\begin{aligned}
d \underline{z}_{V}(\Sigma) & =\left\{d z_{V}^{(1)}(\Sigma), \ldots, d z_{V}^{(r)}(\Sigma)\right\} \text { and } d \underline{z}_{V}\left(\Sigma^{c}\right) \\
& =\left\{d z_{V}^{(r+1)}\left(\Sigma^{c}\right), \ldots, d z_{V}^{(r+s)}\left(\Sigma^{c}\right)\right\}
\end{aligned}
$$

Then $d \underline{z}_{V}:=\left(d \underline{z}_{V}(\Sigma), d \underline{z}_{V}\left(\Sigma^{c}\right)\right)$ form a basis of $\Omega_{\mathcal{A}_{g}(\tau)}$. We define a $d$-tuple $r$-form $\boldsymbol{\omega}_{V / \mathbf{C}}(\Sigma)$ and a $d$-tuple $s$-form $\boldsymbol{\omega}_{V / \mathbf{C}}\left(\Sigma^{c}\right)$ by

$$
\boldsymbol{\omega}_{V / \mathbf{C}}(\Sigma)=\bigwedge_{i=1}^{r} d z_{V}^{(i)}(\Sigma) ; \boldsymbol{\omega}_{V / \mathbf{C}}\left(\Sigma^{c}\right)=\bigwedge_{i=r+1}^{r+s} d z_{V}^{(i)}\left(\Sigma^{c}\right) .
$$

2.5. Igusa schemes associated to $\boldsymbol{U}(\boldsymbol{V})$. For the future application, we have to consider the Igusa schemes associated to the unitary group $U(V)$ as well. Let $C l_{\mathcal{F}}^{+}(K)$ be a set of representatives of the group $\mathcal{F}_{+} \backslash \mathbf{A}_{\mathcal{F}, f}^{\times} / \nu(K)$ in $\mathbf{A}_{\mathcal{F}, f}^{(p)}$. For each $\mathbf{c} \in C l_{\mathcal{F}}^{+}(K)$, we consider the functor $\mathfrak{I}_{K, n, \mathrm{Pol}_{p}, \mathbf{c}}^{(p)}: S C H_{/ \mathcal{O}} \rightarrow S E T S$

$$
\begin{aligned}
& \mathfrak{I}_{K, n, \mathrm{Pol}_{p}, \mathbf{c}}^{(p)}(S) \\
& \quad=\left\{\left(A, \lambda, \iota, \bar{\eta}^{(p)}, j\right)_{S} \mid\left(A, \bar{\lambda}, \iota, \bar{\eta}^{(p)}\right) \in \mathfrak{C}_{K, n}^{(p)}(S), \lambda \text { is a c-polarization }\right\} / \simeq .
\end{aligned}
$$

Here by c-polarization we mean that $\lambda$ is a polarization in the class $\bar{\lambda}$ such that

$$
e^{\lambda}=u \cdot e^{\eta}, u \in \mathbf{c} \nu(K) .
$$

It is shown in [Hid04b, page 136] that the isomorphism class $\left[\left(A, \lambda, \iota, \eta^{(p)} K, j\right)_{S}\right]$ is independent of the choice of $\lambda$ in $\bar{\lambda}$ under the assumption (neat). Then $\mathfrak{I}_{K, n, \operatorname{Pol}_{p}, \mathbf{c}}^{(p)}$ is represented by a scheme over $\mathcal{O}$, which is denoted by $I_{U(V)}^{0}\left(K^{n}, \mathbf{c}\right)_{/ \mathcal{O}}$.

Pick $g_{\mathbf{c}} \in G\left(\mathbf{A}_{\mathcal{F}, f}^{(p)}\right)$ such that $\nu\left(g_{\mathbf{c}}\right)=\mathbf{c}$ and let ${ }^{\mathbf{c}} K^{n}=g_{\mathbf{c}} K^{n} g_{\mathbf{c}}^{-1} \cap U(V)\left(\mathbf{A}_{\mathcal{F}, f}\right)$. We have an isomorphism

$$
M_{U(V)}\left(X^{+},{ }^{\mathbf{c}} K\right) \stackrel{\sim}{\rightarrow} I_{U(V)}^{0}\left(K^{n}, \mathbf{c}\right)_{/ \mathbf{C}}
$$

As explained in [Hid04b, §4.2.1] for the Hilbert modular varieties, we have

$$
\bigsqcup_{\mathbf{c} \in C l_{\mathcal{F}}^{+}(K)} I_{U(V)}^{0}\left(K^{n}, \mathbf{c}\right)_{/ \mathcal{O}}=I_{G}^{0}\left(K^{n}\right) / \mathcal{O} .
$$

We write $I_{U(V)}^{0}\left(K^{n}\right)$ for $I_{U(V)}^{0}\left(K^{n}, \mathbf{c}\right)$ if $\mathbf{c}=1$. 
2.6. Morphisms between Igusa schemes. Let $(W, \vartheta, L)$ and $\left(V, \vartheta_{r, s}, M\right)$ be as before. Put $L^{-1}=L_{\Sigma}:=\left(L \otimes \mathbf{Z}_{p}\right)_{\Sigma}$ and $L^{0}=L_{\Sigma^{c}}:=\left(L \otimes \mathbf{Z}_{p}\right)_{\Sigma^{c}}$. Then $\left\{L^{-1}, L^{0}\right\}$ is a polarization of $L_{p}=L \otimes_{\mathbf{z}} \mathbf{Z}_{p}$. Recall the standard polarization of $M_{p}$ in 1.8 is

$$
M^{-1}=\sum_{i=1}^{s}\left(\mathcal{O}_{\mathcal{K}} \otimes_{\mathbf{Z}} \mathbf{Z}_{p}\right) y^{i}+L^{-1} \text { and } M^{0}=L^{0}+\sum_{i=1}^{s}\left(\mathcal{O}_{\mathcal{K}} \otimes_{\mathbf{Z}} \mathbf{Z}_{p}\right) x^{i}
$$

Let $\underline{V}=\left(V, \vartheta_{r, s}, M, M^{-1} \oplus M^{0}\right)$ and $-\underline{W}=\left(W,-\vartheta, L, L^{0} \oplus L^{-1}\right)$. Let $\left(\mathbf{W}, \eta_{r}\right)=$ $\left.(V \oplus \overline{(}-W), \vartheta_{r, s} \oplus(-\vartheta)\right)$. Let $\mathbf{L}:=M \oplus L$ be an $\mathcal{O}_{\mathcal{K}}$-lattice in $\mathbf{W}$ and let $\left\{\mathbf{L}^{-1}, \mathbf{L}^{0}\right\}=$ $\left\{M^{-1} \oplus L^{0}, M^{0} \oplus L^{-1}\right\}$ be the direct product polarization of $\mathbf{L}_{p}$. Put

$$
\underline{\mathbf{W}}=\underline{V} \oplus(-\underline{W})=\left(\mathbf{W}, \eta_{r}, \mathbf{L}, \mathbf{L}^{-1} \oplus \mathbf{L}^{0}\right) .
$$

Let $G_{1}=U(V), G_{2}=U(W)$ and $G_{3}=U(\mathbf{W})$ be the associated unitary groups. For open compact subgroups $K_{i} \subset G_{i}\left(\mathbf{A}_{\mathbf{Q}, f}\right)$ such that $K_{1} \times K_{2} \subset K_{3}$ and a fixed $c \in\left(\mathbf{A}_{\mathcal{F}, f}^{p}\right)^{\times}$, we write $I_{i}^{0}\left(K_{i}\left(p^{n}\right)\right)$ for the Igusa schemes $I_{G_{i}}^{0}\left(K_{i}\left(p^{n}\right), \mathbf{c}\right)$ associated to unitary groups with additional data. Then we have a natural morphism

$$
i_{V, W}: I_{1}^{0}\left(K_{1}\left(p^{n}\right)\right)_{/ \mathcal{O}} \times I_{2}^{0}\left(K_{2}\left(p^{n}\right)\right)_{/ \mathcal{O}} \longrightarrow I_{3}^{0}\left(K_{3}\left(p^{n}\right)\right)_{/ \mathcal{O}}
$$

defined by

$$
\begin{aligned}
i_{V, W} & {\left[\left(A_{1}, \lambda_{1}, \iota_{1}, \eta_{1}^{(p)} K_{1}, j_{1}\right)\right],\left[\left(A_{2}, \lambda_{2}, \iota_{2}, \eta_{2}^{(p)} K_{2}, j_{2}\right)\right] } \\
= & {\left[\left(A_{1} \times A_{2}, \lambda_{1} \times \lambda_{2}, \iota_{1} \times \iota_{2},\left(\eta_{1}^{(p)} \times \eta_{2}^{(p)}\right) K_{3}, j_{1} \times j_{2}\right)\right] . }
\end{aligned}
$$

Now we consider a different polarization of $\mathbf{L}_{p}$. Let $w^{+, i}\left(\right.$ resp. $\left.w^{-, i}\right)$ be the image of $w^{i}$ in $W$ (resp. $-W$ ) as a subspace in $\mathbf{W}$. Define a basis $\left\{\mathbf{y}^{i}, \mathbf{x}^{i}\right\}_{i=1}^{r}$ of $\mathbf{W}$ by $\mathbf{y}^{i}=y^{i}, \mathbf{x}^{i}=x^{i}$ if $1 \leq i \leq s$ and

$$
\begin{aligned}
& \mathbf{y}^{i}=\frac{1}{2} w^{+, i-s}-\frac{1}{2} w^{-, i-s}, \\
& \mathbf{x}^{i}=w^{+, i-s} \vartheta^{-1}+w^{-, i-s} \vartheta^{-1} \text { if } s<i \leq r .
\end{aligned}
$$

Put $\mathbf{Y}=\sum_{i=1}^{r}\left(\mathcal{O}_{\mathcal{K}} \otimes \mathbf{Z}_{p}\right) \mathbf{y}^{i}$ and $\mathbf{X}=\sum_{i=1}^{r}\left(\mathcal{O}_{\mathcal{K}} \otimes \mathbf{Z}_{p}\right) \mathbf{x}^{i}$. Then $\{\mathbf{Y}, \mathbf{X}\}$ is also a polarization of $\mathbf{L}_{p}=M_{p} \oplus\left(-L_{p}\right)$ and

$$
\mathbf{L}_{\Sigma}=M_{\Sigma} \oplus(-L)_{\Sigma}=\mathbf{Y}_{\Sigma} \oplus \mathbf{X}_{\Sigma}
$$

We let $\Upsilon$ be the unique element in $\operatorname{GL}\left(\mathbf{W}_{\Sigma}\right)=\prod_{v \in \Sigma_{p}} \operatorname{GL}_{2 r}\left(\mathcal{F}_{v}\right) \simeq U(\mathbf{W})\left(\mathcal{F}_{p}\right)$ such that $\mathbf{y}^{i} \Upsilon=\mathbf{y}^{i}, \mathbf{x}^{i} \Upsilon=\mathbf{x}^{i}$ if $1 \leq i \leq s$ and $w_{\Sigma}^{+, i-s} \Upsilon=\mathbf{y}_{\Sigma}^{i}$ and $w_{\Sigma}^{-, i-s} \Upsilon=\mathbf{x}_{\Sigma}^{i}$ if $s<i \leq r$. Then $\mathbf{L}_{\Sigma}^{0} \Upsilon=\mathbf{X}_{\Sigma}$ and $\mathbf{L}_{\Sigma}^{-1} \Upsilon=\mathbf{Y}_{\Sigma}$. The matrix representation of $\Upsilon$ according to the basis $\left\{\mathbf{y}_{\Sigma}^{i}, \mathbf{x}_{\Sigma}^{i}\right\}$ is

$$
\Upsilon=\left[\begin{array}{cccc}
\mathbf{1}_{s} & & & \\
& \frac{1}{2} & & -\frac{1}{2} \\
& \vartheta^{-1} & & \vartheta^{-1}
\end{array}\right] \in U(\mathbf{W})\left(\mathcal{F}_{p}\right) .
$$

We give an explicit expression of the morphism $i_{V, W}$ in (2.12) in terms of the complex coordinates defined in (2.9). Notation is as in $\$ 2.4 .2$ By the definition of $\mathbf{C}^{r, r}$ we can decompose

$$
\mathbf{C}^{r, r}=\mathbf{C}^{s}\left(\Sigma^{c}\right) \oplus \mathbf{C}^{r-s}\left(\Sigma^{c}\right) \oplus \mathbf{C}^{s}(\Sigma) \oplus \mathbf{C}^{r-s}(\Sigma)=\mathbf{C}^{r, s} \oplus \mathbf{C}^{0, r-s} .
$$


For $\tau=\left[\begin{array}{l}x \\ y\end{array}\right] \in X_{r, s}$, we let $i_{\tau} \in \mathrm{GL}\left(\mathbf{C}^{r, r}\right)$ be the matrix such that according to the above decomposition

and put

$$
i_{\tau}=\left[\begin{array}{cccc}
\mathbf{1}_{s} & & & \\
& \mathbf{1}_{r-s} & & \\
& & \mathbf{1}_{s} & \\
& & \vartheta^{-1} y & \mathbf{1}_{r-s}
\end{array}\right]
$$

$$
Z_{\tau}=\left[\begin{array}{cc}
x & 0 \\
y & -2^{-1} \vartheta
\end{array}\right] \in X_{r, r} .
$$

For $g \in U(V)$ and $h \in U(W)$, a straightforward computation shows that

$$
\left(\mathbf{L}_{(g, h)}\left(Z_{\tau}\right)\right) i_{\tau}=M_{[g]}(\tau) \oplus L_{h}\left(\mathbf{x}_{0}\right) .
$$

Hence, $i_{\tau}$ induces an isomorphism

$$
\mathcal{A}(\mathbf{W})_{(g, h)}\left(Z_{\tau}\right)=\frac{\mathbf{C}^{r, r}}{\mathbf{L}_{[(g, h)]}\left(Z_{\tau}\right)} \stackrel{i_{\tau}}{\rightarrow} \frac{\mathbf{C}^{r, s}}{M_{[g]}(\tau)} \bigoplus \frac{\mathbf{C}^{0, r-s}}{L_{[h]}\left(\mathbf{x}_{0}\right)}=\mathcal{A}(V)_{g}(\tau) \times \mathcal{A}(W)_{h}\left(\mathbf{x}_{0}\right),
$$

and

$$
i_{\tau}^{*}\left(\boldsymbol{\omega}_{V / \mathbf{C}}(\Sigma), \omega_{V / \mathbf{C}}\left(\Sigma^{c}\right) \wedge \boldsymbol{\omega}_{W / \mathbf{C}}\left(\Sigma^{c}\right)\right)=\left(\boldsymbol{\omega}_{\mathbf{W} / \mathbf{C}}(\Sigma), \boldsymbol{\omega}_{\mathbf{W} / \mathbf{C}}\left(\Sigma^{c}\right)\right) .
$$

In view of (2.9) and (2.16), the morphism $i_{V, W}$ in (2.12) over $\mathbf{C}$ is given by

$$
i_{V, W}\left([\tau, g],\left[\mathbf{x}_{0}, h\right]\right)=\left[Z_{\tau},(g, h) \Upsilon\right] .
$$

Here the appearance of $\Upsilon \in U(\mathbf{W})\left(\mathcal{F}_{p}\right)$ given in (2.14) is due to the change of level structures at $p$ obtained from the direct product polarization $\left\{\mathbf{L}^{-1}, \mathbf{L}^{0}\right\}$ to the standard polarization $\{\mathbf{Y}, \mathbf{X}\}$ when $G U(\mathbf{W})$ is regarded as the standard matrix group $G U(r, r)$.

2.7. Compactifications. We collect some basic facts in the theory of the toroidal and minimal compactifications of unitary Shimura varieties. For details and proofs, the reader is referred to [FC90], Fuj89] and [Lan08]. We shall follow the exposition in [SU13, §5] but restrict ourselves to the simple case $s=1\left(\right.$ so $\operatorname{dim} X_{\mathcal{K}}=\operatorname{dim} Y_{\mathcal{K}}=$ 1 and $\left.G=G U(r, 1)_{/ \mathcal{F}}\right)$. Let $P$ be the stabilizer of the flag $\{0\} \subset X_{\mathcal{K}} \subset V$ in $G=G U(V)$. Then $P$ is the standard parabolic subgroup of $G$. Let $N_{P}$ be the unipotent radical of $P$ and let

$$
M_{P}:=\mathrm{GL}\left(X_{\mathcal{K}}\right) \times G U(W) \hookrightarrow G U(V),\left(a, g_{1}\right) \mapsto \operatorname{diag}\left(a, g_{1}, \nu\left(g_{1}\right) a^{-*}\right)
$$

be the standard Levi subgroup of $P$. Let $G_{P}:=G U(W) \hookrightarrow G U(V), g_{1} \mapsto$ $\operatorname{diag}\left(1, g_{1}, \nu\left(g_{1}\right)\right)$.

2.7.1. Cusps. The set of cusp labels for $S_{G}(K)$ is defined to be the double coset

$$
\underline{C}(K):=\left(\mathrm{GL}\left(X_{\mathcal{K}}\right) \times G_{P}\left(\mathbf{A}_{\mathcal{F}, f}\right)\right) N_{P}\left(\mathbf{A}_{\mathcal{F}, f}\right) \backslash G\left(\mathbf{A}_{\mathcal{F}, f}\right) / K .
$$

Since $G_{P}$ is a totally definite unitary group, the set $\underline{C}(K)$ is finite. For $g \in G\left(\mathbf{A}_{\mathcal{F}, f}\right)$, we denote by $[g]$ the class in $\underline{C}(K)$. By the strong approximation and Iwasawa decomposition $G\left(\mathbf{A}_{\mathcal{F}, f}\right)=P\left(\overline{\mathbf{A}}_{\mathcal{F}, f}\right) K^{0}$, we can choose a convenient set $C(K)$ of representatives of $\underline{C}(K)$ in $G\left(\mathbf{A}_{\mathcal{F}, f}^{(p)}\right)$ such that $g=p k^{0}$ for $p \in P\left(\mathbf{A}_{\mathcal{F}, f}^{\left(p N_{0}\right)}\right)$ and $k^{0} \in K^{0}$ and with the similitude $\nu(g) \in \widehat{O}$. 
2.7.2. Toroidal compactifications. The existence of toroidal compactifications of Siegel modular varieties has been established in [FC90]. For general Shimura varieties of PEL-type, these are done in the works of [Fuj89 and [Lan08]. To a datum of smooth rational cone decompositions $\left\{\xi_{[g]}\right\}_{g \in C(K)}$ of $\mathcal{F}_{+}$, we can attach the toroidal compactification $\bar{S}_{G}(K)_{/ \mathcal{O}}$ of $S_{G}(K)_{/ \mathcal{O}}$, which is a proper smooth scheme over $\mathcal{O}$ containing $S_{G}(K)$ as an open dense subscheme. The complement of $S_{G}(K)$ is a relative Cartier divisor with normal crossings. In addition, there is a quadruple $\mathcal{G}=(\mathcal{G}, \lambda, \iota, \eta)$ over $\bar{S}_{G}(K)$, where $\mathcal{G}$ is a semi-abelian scheme with an $\mathcal{O}_{\mathcal{K}}$-action by $\iota$ and a homomorphism $\lambda: \mathcal{G} \rightarrow \mathcal{G}^{t}$ such that $\left.\underline{\mathcal{G}}\right|_{S_{G}(K)}=\underline{\mathcal{A}}$, and the universal quadruple over $S_{G}(K)$ and $\eta$ is the level structure in the quadruple $\underline{\mathcal{A}}$.

2.7.3. The minimal compactification. Let $\underline{\omega}:=e^{*} \Omega_{\mathcal{G}_{\bar{S}_{G}(K)}}$ be the pull-back of the sheaf of the relative Kähler differentials $\Omega_{\mathcal{G}_{\bar{S}_{G}(K)}}$ along the zero section $e$ of $\mathcal{G}_{/ \bar{S}_{G}(K)}$. Then $\underline{\omega}$ is a locally free coherent $\mathcal{O}_{\bar{S}_{G}(K)}$-module. The minimal compactification $S_{G}^{*}(K)_{/ \mathcal{O}}$ of $S_{G}(K)$ is defined to be

$$
S_{G}^{*}(K):=\operatorname{Proj} \bigoplus_{k=0}^{\infty} \Gamma\left(\bar{S}_{G}(K), \operatorname{det} \underline{\omega}^{k}\right) .
$$

The O-schemes $S_{G}(K), \bar{S}_{G}(K)$ and $S_{G}^{*}(K)$ fit in the following diagram:

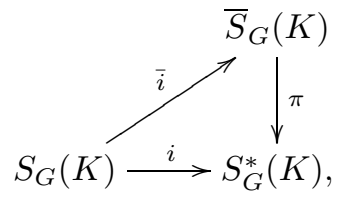

where $i$ and $\bar{i}$ are open immersions, and $\pi$ is a blow-down map. The following results about the minimal compactification $S_{G}^{*}(K)$ are due to Kai-Wen Lan in his Harvard thesis [Lan08].

\section{Theorem 2.4.}

(1) $\pi_{*} \operatorname{det} \underline{\omega}$ is an ample line bundle on $S_{G}^{*}(K)$ and $S_{G}^{*}(K)$ is a normal projective scheme of finite type over $\mathcal{O}$.

(2) $\pi_{*} \mathcal{O}_{\bar{S}_{G}(K)}=\mathcal{O}_{S_{G}^{*}(K)}$. Hence $\pi$ has geometrically connected fibres.

(3) $S_{G}^{*}(K)(\mathbf{C})$ is the classical Satake-Baily-Borel compactification.

(4) There is a natural stratification of $\partial S_{G}^{*}(K):=S_{G}^{*}(K) \backslash S_{G}(K)$ indexed by $C(K)$ :

$$
\partial S_{G}^{*}(K)=\bigsqcup_{g \in C(K)} S_{G_{P}}\left(K_{P}^{g}\right), K_{P}^{g}=G_{P}\left(\mathbf{A}_{\mathcal{F}, f}\right) \cap g K g^{-1} .
$$

If $K_{p} \neq K_{p}^{0}$, currently we only have toroidal compactifications $\bar{S}_{G}(K) / \mathcal{K}$ and the minimal compactification $S_{G}^{*}(K)_{/ \mathcal{K}}$ of $S_{G}(K)_{/ \mathcal{K}}$ over $\mathcal{K}=\mathcal{O}[1 / p]$. If we choose compatible rational cone decompositions, then the maps $S_{G}\left(K_{\bullet}^{n}\right) / \mathcal{K} \rightarrow S_{G}(K) / \mathcal{K}$ extend to these compactifications over $\mathcal{K}$.

2.7.4. Local charts and Mumford families. Let $g \in G\left(\mathbf{A}_{\mathcal{F}, f}\right)$ with $g_{p} \in K_{p}^{0}$. We write $g^{\vee}=k g_{i}^{\vee} \gamma$ with $k \in K, g_{i} \in C(K)$ and $\gamma \in G(\mathcal{F})^{+}$(recall that $g^{\vee}=g^{-1} \nu(g)$ ). Let $Y_{g}=Y g_{i}^{\vee}$ and $X_{g}^{\vee}=X^{\vee} g_{i}^{\vee}$. Then $Y_{g}=\mathfrak{b} y^{1}$ and $X_{g}^{\vee}=\mathfrak{a}^{-1} \mathfrak{d}_{\mathcal{K}}^{-1} x^{1}$ for some fractional ideals $\mathfrak{a}$ and $\mathfrak{b}$ of $\mathcal{O}_{\mathcal{K}}$. Let $X_{g}=\left\{y \in I_{Y} \mid\left\langle y, X_{g}^{\vee}\right\rangle_{r, 1} \subset \mathbf{Z}\right\}=c(\mathfrak{a}) y^{1}$ be 
the $\mathbf{Z}$-dual of $X_{g}^{\vee}$ ( $c$ is the complex conjugation). With our choice of $Y=\mathcal{O}_{\mathcal{K}} y^{1}$, $X^{\vee}=\mathfrak{d}_{\mathcal{K}}^{-1} x^{1}$ and $g_{i} \in C(K),\left\langle Y_{g}, X_{g}^{\vee}\right\rangle \subset \mathbf{Z}$, so $\mathfrak{b} \subset c(\mathfrak{a})$. Let $i: Y_{g}=\mathfrak{b} y^{1} \hookrightarrow X_{g}=$ $c(\mathfrak{a}) y^{1}$ be the inclusion map. Let $I_{g}$ be the subgroup in $X_{g} \otimes_{\mathbf{z}} Y_{g}$ generated by

$$
i\left(y^{\prime}\right) \otimes y-i(y) \otimes y^{\prime} ; x b \otimes y-x \otimes c(b) y \quad\left(x \in X_{g}, y, y^{\prime} \in Y_{g}, b \in \mathcal{O}_{\mathcal{K}}\right) .
$$

Let $\mathscr{S}_{g}=S\left(X_{g} \otimes_{\mathbf{z}} Y_{g}\right)$ be the maximal free quotient of the group $X_{g} \otimes_{\mathbf{Z}} Y_{g} / I_{g}$. The $\mathbf{Z}$-dual $\mathscr{S}_{[g]}^{\vee}=\operatorname{Hom}_{\mathbf{Z}}\left(\mathscr{S}_{[g]}, \mathbf{Z}\right)$ is the space of $\mathbf{Z}$-valued symmetric and Hermitian bilinear forms on $c(\mathfrak{a}) \times \mathfrak{b}$, so $\mathscr{S}_{[g]}^{\vee}$ is isomorphic to $\left(\mathfrak{a} \mathfrak{b} \mathfrak{d}_{\mathcal{K}}\right)^{-1} \cap \mathcal{F}$, and $\mathscr{S}_{[g]}$ is the fractional ideal $\left(\mathfrak{a b d} \mathfrak{d}_{\mathcal{K}} \cap \mathcal{F}\right) \mathfrak{d}_{\mathcal{F}}^{-1}$ of $\mathcal{F}$. Let $\operatorname{Hom}(\mathcal{F}, \mathbf{Q})$ be the space of $\mathbf{Q}$-linear maps from $\mathcal{F}$ to $\mathbf{Q}$ and choose a set of $d$ linear independent elements $\xi=\left\{l_{1}, \ldots, l_{d}\right\}$ in $\operatorname{Hom}(\mathcal{F}, \mathbf{Q})$ such that $l_{i}\left(\mathcal{F}_{+}\right) \subset \mathbf{Q}_{+}$for all $i$. Let $N_{0}$ be the level of $K$ as in (neat). Set $\mathscr{S}_{[g]}^{\xi}=\left\{s \in N_{0}^{-1} \mathscr{S}_{[g]} \mid l(s) \geq 0, l \in \xi\right\}, \mathscr{S}_{[g]}^{+}=\left\{s \in N_{0}^{-1} \mathscr{S}_{[g]} \mid s \in \mathcal{F}_{+}\right\}$ and $\mathscr{S}_{[g]}^{0}=\mathscr{S}_{[g]}^{+} \cup\{0\}$.

Let $S_{[g]}:=S_{G_{P}}\left(K_{P}^{g}\right)$ and let $\underline{\mathcal{B}}=\left(\mathcal{B}, \bar{\lambda}_{\mathcal{B}}, \iota_{\mathcal{B}}, \eta_{\mathcal{B}}\right)$ be the universal quadruple over $S_{[g]}$. Let $\mathcal{P}$ be the Poincaré line bundle over $\mathcal{B} \times \mathcal{B}^{t}$. Define the group scheme $\mathcal{Z}_{[g]}$ over $S_{[g]}$ by

$$
\begin{aligned}
\mathcal{Z}_{[g]} & =\underline{\operatorname{Hom}}_{\mathcal{O}_{\mathcal{K}}}\left(X_{g}, \mathcal{B}^{t}\right) \times_{\underline{\operatorname{Hom}}_{\mathcal{O}_{\mathcal{K}}}\left(Y_{g}, \mathcal{B}^{t}\right)} \underline{\operatorname{Hom}}_{\mathcal{O}_{\mathcal{K}}}\left(Y_{g}, \mathcal{B}\right) \\
& =\left\{\left(c, c^{t}\right) \in \underline{\operatorname{Hom}}_{\mathcal{O}_{\mathcal{K}}}\left(X_{g}, \mathcal{B}^{t}\right) \times \underline{\operatorname{Hom}}_{\mathcal{O}_{\mathcal{K}}}\left(Y_{g}, \mathcal{B}\right) \mid c(i(y))=\lambda\left(c^{t}(y)\right), y \in Y_{g}\right\} .
\end{aligned}
$$

Let $\mathcal{Z}_{[g]}^{\circ}$ be the connected component of $Z$. Each $\beta \in X \otimes Y$ induces a tautological map from $\mathbf{c}(\beta): \mathcal{Z}_{[g]}^{\circ} \rightarrow \mathcal{B} \times \mathcal{B}^{t}$. We let $\mathcal{L}(\beta)=\mathbf{c}(\beta)^{*} \mathcal{P}$ be the line bundle over $\mathcal{Z}_{[g]}^{\circ}$ obtained by the pull-back of $\mathcal{P}$ via $\mathbf{c}(\beta)$. Because of the symmetry of the polarization $\lambda$ and the ampleness of $\mathcal{P}, \mathcal{L}(\beta)$ only depends on the holomorphic image of $\beta$ in $\mathscr{S}_{[g]}$.

For $\bullet=0,1$ or $\emptyset$, we let $K_{P, \bullet}^{g, n}:=g K_{\bullet}^{n} g^{-1} \cap G_{P}\left(\mathbf{A}_{\mathcal{F}, f}\right)$ and let $I_{[g]}\left(K_{\bullet}^{n}\right):=$ $I_{G_{P}}\left(K_{P, \bullet}^{g, n}\right)$ be the associated Igusa scheme over $S_{[g]}$. In our simple situation, $S_{[g]}$ and $I_{[g]}\left(K_{\bullet}^{n}\right)$ are affine schemes of finite type over $\mathcal{O}$. Let $A_{[g]}\left(\right.$ resp. $\left.A_{[g]}^{n}\right)$ be the coordinate ring of $S_{[g]}$ (resp. $\left.I_{[g]}\left(K_{1}^{n}\right)\right)$. For $\bullet=\xi, 0$ or + , we write $\mathscr{S}_{g, n}$ for $p^{-n} \mathscr{S}_{[g]}^{\bullet}$ and let $\mathcal{R}_{[g], n}^{\bullet}$ be the $A_{[g]}^{n} \llbracket \llbracket \mathscr{S}_{g, n}^{\bullet} \rrbracket$-algebra defined by

$$
\begin{aligned}
\mathcal{R}_{[g], n}^{\bullet} & :=\prod_{\beta \in \mathscr{S}_{\boldsymbol{g}, n}} H^{0}\left(\mathcal{Z}_{[g] / I_{[g]}\left(K_{1}^{n}\right)}^{\circ}, \mathcal{L}(\beta)\right) q^{\beta} \\
& =\prod_{\beta \in \mathscr{S}_{\boldsymbol{g}_{, n}}} A_{[g]}^{n} \otimes_{A_{[g]}} H^{0}\left(\mathcal{Z}_{[g]}^{\circ}, \mathcal{L}(\beta)\right) q^{\beta} .
\end{aligned}
$$

Write $\mathcal{R}_{[g]}^{\bullet}=\mathcal{R}_{[g], 0}^{\bullet}$. Let $\mathcal{J}_{+}$be the ideal of $\mathcal{R}_{[g], n}^{\bullet}$ generated by $\left\{q^{\beta}\right\}_{\beta \in \mathscr{S}_{[g]}^{+}}$. By Mumford's construction ([Cha85] and [FC90]), there exists a semi-abelian scheme $\left(\mathcal{M}_{[g]}, \iota_{\mathcal{M}}\right)$ over Spec $\mathcal{R}_{[g]}^{\xi}$ together with an $\mathcal{O}_{\mathcal{K}}$-action $\iota_{\mathcal{M}}: \mathcal{O}_{\mathcal{K}} \rightarrow$ End $\mathcal{M} \otimes_{\mathbf{Z}} \mathbf{Z}_{(p)}$ such that $\mathcal{M}$ is an abelian scheme over $\operatorname{Spec} \mathcal{R}_{[g]}^{\xi}\left[1 / \mathcal{J}_{+}\right]$, and $\tilde{\mathcal{M}}:=\mathcal{M} \otimes \mathcal{R}_{[g]}^{\xi} / \mathcal{J}_{+}$is the universal $\mathcal{O}_{\mathcal{K}}$-Raynaud extension over $\mathcal{Z}_{[g]}^{\circ}$ with the exact sequence

$$
0 \longrightarrow X_{g}^{\vee} \otimes \mathbb{G}_{m} \longrightarrow \tilde{\mathcal{M}} \longrightarrow \mathcal{B} \longrightarrow 0 .
$$

The datum $\left(\bar{\lambda}_{\mathcal{B}}, K_{P}^{g} \eta_{\mathcal{B}}\right)$ induces a polarization and a level structure $\left(\bar{\lambda}_{\mathcal{M}}, \bar{\eta}_{\mathcal{M}}^{(p)}\right)$ of $\mathcal{M}$ over $\mathcal{R}_{[g]}^{\xi}$ compatible with the degeneration (cf. [FC90, IV. 6.4, 6.5 and V. 2.5(5)]). 
The Mumford quadruple $\underline{\mathcal{M}}_{[g]}$ at the cusp $[g]$ is the $\mathcal{R}_{[g]}^{\xi}$-quadruple of level $K$ given by

$$
\underline{\mathcal{M}}_{[g]}=\left(\mathcal{M}_{[g]}, \bar{\lambda}_{\mathcal{M}}, \iota_{\mathcal{M}}, \bar{\eta}_{\mathcal{M}}^{(p)}\right)_{/ \mathcal{R}_{[g]}^{\xi}} .
$$

Moreover, there is a morphism $\iota_{[g]}^{\xi}: \operatorname{Spec} \mathcal{R}_{[g]}^{\xi} \rightarrow \bar{S}_{G}(K)$ such that $\left(\iota_{[g]}^{\xi}\right) * \underline{\mathcal{G}}=\underline{\mathcal{M}}_{[g]}$. Let $\Gamma_{[g]}=\operatorname{GL}\left(X_{\mathcal{K}}\right) \cap g_{i} K g_{i}^{-1}$. Then the group $\Gamma_{[g]} \subset \mathcal{O}_{\mathcal{F}}^{\times}$acts on $X_{g}^{\vee}$, and thus induces an automorphism of $\mathcal{\mathcal { M }}_{[g]}$ by the functoriality of Mumford's construction.

Let $\left(\underline{\mathcal{B}}, j_{\mathcal{B}}\right)$ be the universal quintuple over $I_{[g]}\left(K^{n}\right)$. Fixing a $p^{n}$-level structure $j_{\mathcal{M}}$ on $\underline{\mathcal{M}}_{[g]}$ lifted from the tautological $p^{n}$-level structure $j_{\mathcal{B}}$ on $\mathcal{B}$ and the natural one on $X^{\vee} \otimes \mathbb{G}_{m}$, we obtain the Mumford quintuple $\left(\underline{\mathcal{M}}_{[g]}, j_{\mathcal{M}}\right)$ over $\mathcal{R}_{[g], n}^{\xi}$.

2.7.5. The local structure of $\partial S_{G}^{*}(K)$. We have the following description of the local structure of $\partial S_{G}^{*}(K)$ at a cusp $[g]$. It is similar to the Siegel case [FC90, V.2.7] (cf. [SU13, Thm. 5.3]).

Proposition 2.5 (Prop.7.2.3.11 Lan08]). The completion of the local ring of $\mathcal{O}_{S_{G}^{*}(K), \bar{x}}$ at a geometric point $\bar{x}$ in $S_{[g]}$ is given by

$$
\begin{aligned}
\mathcal{O}_{S_{G}^{*}(K), \bar{x}}^{\wedge} & =\left(\pi_{*} \mathcal{O}_{\bar{S}_{G}(K)}\right)_{\bar{x}}^{\wedge}=H^{0}\left(\Gamma_{[g]}, \mathcal{R}_{[g]}\right) \\
& =\left\{\sum_{\beta \in \mathscr{S}_{[g]}^{0}} \mathbf{a}(\beta) q^{\beta} \in \mathcal{R}_{[g]} \mid \mathbf{a}\left(\beta \epsilon^{2}\right)=\mathbf{a}(\beta), \forall \epsilon \in \Gamma_{[g]}\right\} .
\end{aligned}
$$

2.7.6. Igusa schemes over $\bar{S}_{G}(K)$. Let $n$ be a positive integer. The Igusa scheme $I_{G}\left(K^{n}\right)$ over $\bar{S}_{G}(K)$ is the scheme that represents the functor

$$
\underline{I}_{G}\left(K^{n}\right)=\underline{\operatorname{Inj}}_{\mathcal{O}_{\mathcal{K}}}\left(\boldsymbol{\mu}_{p^{n}} \otimes \mathbf{z} M^{0}, \mathcal{G}\right) .
$$

For $n^{\prime} \geq n$, let $\pi_{n^{\prime}, n}: I_{G}\left(K_{n^{\prime}}\right) \rightarrow I_{G}\left(K^{n}\right)$ be the natural morphism induced by the inclusion $M^{0} \otimes \boldsymbol{\mu}_{p^{n}} \hookrightarrow M^{0} \otimes \boldsymbol{\mu}_{p^{n^{\prime}}}$. The forgetful morphism $\pi_{n}: I_{G}\left(K^{n}\right) \longrightarrow \bar{S}_{G}(K)$ defined by $\pi:(\underline{A}, j) \mapsto \underline{A}$ is étale. Hence $I_{G}\left(K^{n}\right)$ is smooth over Spec $\mathcal{O}$. Let $\mathbf{H}:=\mathrm{GL}_{\mathcal{O}_{\mathcal{K}}}\left(M^{0}\right)=\mathrm{GL}_{O}\left(M_{\Sigma}^{0}\right) \times \mathrm{GL}_{O}\left(M_{\Sigma^{c}}^{0}\right)$ and let $\mathbf{H}$ act on $I_{G}\left(K^{n}\right)$ over $\bar{S}_{G}(K)$ by $h \cdot j\left(m^{0}\right)=j\left(m^{0} \cdot h\right)$. Let $\mathbf{N}\left(\right.$ resp. B) be the stabilizer of gr ${ }^{\bullet} M^{0}\left(\right.$ resp. Fil $\left.{ }^{\bullet} M^{0}\right)$ in $\mathbf{H}$. Define

$$
I_{G}\left(K_{1}^{n}\right):=I_{G}\left(K^{n}\right) / \mathbf{N} \text { and } I_{G}\left(K_{0}^{1}\right):=I_{G}\left(K^{n}\right) / \mathbf{B} .
$$

2.8. CM abelian varieties and periods. We briefly recall the notion of complex and $p$-adic periods associated to CM fields. Consider the special case $s=0$. Then $G=G U(W)$ is a totally definite unitary group of degree one. It follows that $S_{G}(K)$ is finite over $\mathcal{O}$ and $S_{G}(K)(\mathbf{C})$ is a finite set. Let $\underline{\mathcal{B}}$ be the universal quadruple over $S_{G}(K)$ with the structure morphism $\pi: \underline{\mathcal{B}} \rightarrow S_{G}(K)$. Replacing $\mathcal{O}$ by a finite flat unramified extension of $\mathcal{O}$, we may further assume that $S_{G}(K)(\mathcal{O})=$ $G(\mathcal{F})^{+} \backslash G\left(\mathbf{A}_{\mathcal{F}, f}\right) / K$ and $\underline{\mathcal{B}}=\bigsqcup_{[h] \in S_{G}(K)(\mathcal{O})} \underline{\mathcal{B}}_{h}$. By the complex uniformization constructed in $\$ 2.4 .2$, we have

$$
\mathcal{B}_{h}(\mathbf{C})=\frac{\mathbf{C}\left(\Sigma^{c}\right)}{\overline{L_{[h]}} \vartheta} \text { for all } h \in G\left(\mathbf{A}_{\mathcal{F}, f}\right) .
$$

Let $(\underline{\mathcal{B}}, j)$ be the universal quintuple over $S_{G}\left(K^{n}\right) / \mathbf{C}$. Since $\mathcal{B}_{h}$ is an abelian variety with $\mathrm{CM}$ by $\mathcal{O}_{\mathcal{K}}$ and the CM type $\Sigma$ is p-ordinary, it follows that $\mathcal{B}_{h} \otimes_{\mathcal{O}} \overline{\mathbb{F}}_{p}$ 
is an ordinary abelian variety, and $j_{\zeta}$ descends to a level $p^{n}$-structure over a finite unramified extension of $\mathcal{O}^{\prime}$. This in particular implies that $I_{G}\left(K^{n}\right)\left(\mathcal{W}_{p}\right)=$ $G(\mathcal{F})^{+} \backslash G\left(\mathbf{A}_{\mathcal{F}, f}\right) / K^{n}$.

Let $\mathcal{S}=S_{G}(K)_{/ \mathcal{O}}$ and let $\mathcal{H}_{\mathcal{B}}:=\pi_{*}\left(\Omega_{\mathcal{B} / \mathcal{S}}\right)$ be a free $e^{+}\left(\mathcal{O}_{\mathcal{K}} \otimes_{\mathbf{Z}} \mathcal{O}_{\mathcal{S}}\right)$-module of rank $r$. Then $\mathcal{B} / \mathbf{C}$ carries the $e^{+}\left(\mathcal{O}_{\mathcal{K}} \otimes_{\mathbf{z}} \mathcal{O}_{\mathcal{S} / \mathbf{C}}\right)$-basis $d \underline{z}_{W}=d \underline{z}_{W}(\Sigma)$ of $\mathcal{H}_{\mathcal{B}} \otimes_{\mathcal{O}_{\mathcal{S}}, \iota_{\infty}}$ $\mathcal{O}_{\mathcal{S} / \mathrm{C}}$ introduced in (2.10). On the other hand, let $\left(\underline{\mathcal{B}}_{\mathcal{I}_{\infty}}, j_{\mathcal{I}_{\infty}}\right)$ be the universal quintuple sitting over $\mathcal{I}_{\infty}:=\lim _{n} I_{G}\left(K^{n}\right) / \mathcal{W}_{p}$, and then the universal $p^{\infty}$-structure $j_{\mathcal{I}_{\infty}}$ of $\mathcal{B}_{\mathcal{I}_{\infty}}$ gives rise to a $e^{+}\left(\mathcal{O}_{\mathcal{K}} \otimes_{\mathbf{z}} \mathcal{O}_{\mathcal{I}_{\infty}}\right)$-basis $d^{\times} t$ of $\mathcal{H}_{\mathcal{B}} \otimes_{\mathcal{O}_{\mathcal{S}}, \iota_{p}} \mathcal{O}_{\mathcal{I}_{\infty}}$. It follows from the fact that CM abelian schemes $\left\{\left(\underline{\mathcal{B}}_{h}, j_{h}\right)\right\}_{[h] \in \mathcal{I}_{\infty}\left(\mathcal{W}_{p}\right)}$ are prime-to- $p$ isogenous to each other that there exist an $e^{+}\left(\mathcal{O}_{\mathcal{K}} \otimes_{\mathbf{Z}} \mathcal{O}_{\mathcal{S}}\right)$-basis $\boldsymbol{\omega}_{\mathcal{B}}$ of $\mathcal{H}_{\mathcal{B}}$ and a pair $\left(\Omega_{G, \infty}, \Omega_{G, p}\right) \in \mathrm{GL}_{r}\left(e^{+}\left(\mathcal{O}_{\mathcal{K}} \otimes_{\mathbf{z}} \mathbf{C}\right)\right) \times \mathrm{GL}_{r}\left(e^{+}\left(\mathcal{O}_{\mathcal{K}} \otimes \mathbf{z} \mathcal{W}_{p}\right)\right)=\mathrm{GL}_{r}\left(\mathbf{C}^{\Sigma}\right) \times \mathrm{GL}_{r}\left(\mathcal{W}_{p}^{\Sigma}\right)$ such that

$$
\Omega_{G, \infty} d \underline{z}_{W}=\omega_{\mathcal{B}}=\Omega_{G, p} d^{\times} t .
$$

When $r=1$ and $G=\mathcal{K}^{\times}$, we let $\Omega_{\infty}:=\Omega_{G, \infty} \in\left(\mathbf{C}^{\times}\right)^{\Sigma}$ (resp. $\Omega_{p}:=\Omega_{G, p} \in$ $\left(\mathcal{W}_{p}^{\times}\right)^{\Sigma}$ ) be the complex CM period (resp. $p$-adic CM period) of $(\mathcal{K}, \Sigma)$. We remark that up to a $p$-adic unit, $\left(\Omega_{\infty}, \Omega_{p}\right)$ are precisely the periods defined in HT93, (4.4 a, b), page 211] $(c f .(\Omega, c)$ in [Kat78, (5.1.46), (5.1.48)]).

\section{MOdUlar FORMS ON UNITARY GROUPS}

3.1. Rational representations of $\mathbf{G L}_{\boldsymbol{r}} \times \mathbf{G L}_{\boldsymbol{s}}$. Let $R$ be an $\mathcal{O}$-algebra. For an $(r+s)$-tuple $k=\left(a_{1}, \ldots a_{r} ; b_{1}, \ldots b_{s}\right) \in \mathbf{Z}^{r+s}$, we consider the schematical induction module $L_{k}(R)$ given by

$$
L_{k}(R)=\left\{f \in R\left[\mathrm{GL}_{r} \times \mathrm{GL}_{s}\right] \mid f\left(t n_{+} g\right)=k^{-1}(t) f(g), t \in T_{r} \times T_{s}, n_{+} \in N_{r} \times N_{s}^{t}\right\},
$$

where $R\left[\mathrm{GL}_{r} \times \mathrm{GL}_{s}\right]$ denotes the polynomial functions on $\mathrm{GL}_{r} \times \mathrm{GL}_{s}$ with coefficients in $R$ and $k$ is regarded as an algebraic character on $T_{r} \times T_{s}$ defined by

$$
k\left(\operatorname{diag}\left(t_{1}, \ldots, t_{r}\right), \operatorname{diag}\left(t_{s+1}, \ldots, t_{r+s}\right)\right)=t_{1}^{a_{1}} \cdots t_{r}^{a_{r}} \cdot t_{s+1}^{b_{1}} \cdots t_{r+s}^{b_{s}} .
$$

Then $L_{k}(R)$ is a free $R$-module and is the algebraic representation of $\mathrm{GL}_{r}(R) \times \mathrm{GL}_{s}(R)$ with the minimal weight $-k$ with respect to $N_{r} \times N_{s}^{t}$.

Let $H:=\mathrm{R}_{O / \mathbf{z}} \mathrm{GL}_{r} \times \mathrm{R}_{O / \mathbf{z}} \mathrm{GL}_{s}$. Let $T=\mathrm{R}_{O / \mathbf{z}} T_{r} \times \mathrm{R}_{O / \mathbf{z}} T_{s}$ be the diagonal torus of $H$ and let $N_{H}=\mathrm{R}_{O / \mathbf{z}} N_{r} \times \mathrm{R}_{O / \mathbf{z}} N_{s}^{t}$ be a unipotent subgroup. A weight $\underline{k}$ of $T$ is an algebraic character of $T_{/ \mathcal{O}}$, which can be written as an $(r+s)$-tuple $\underline{k}=\left(a_{1}, a_{2}, \ldots, a_{r} ; b_{1}, b_{2} \ldots, b_{s}\right) \in \mathbf{Z}[\Sigma]^{r+s}$ with $a_{i}=\sum a_{i, \sigma} \sigma$ for $i=1, \ldots, r$ and $b_{j}=\sum b_{j, \sigma} \sigma$ for $j=1, \ldots, s$. We say $\underline{k}$ is dominant with respect to $N_{H}$ if $a_{1, \sigma} \geq \cdots \geq a_{r, \sigma} \geq-b_{1, \sigma} \geq \cdots \geq-b_{s, \sigma}$ for all $\sigma \in \Sigma$. Put

$$
\|\underline{k}\|:=a_{1}+\cdots+a_{r}+b_{1}+\cdots+b_{s} \in \mathbf{Z}[\Sigma] .
$$

For $n \in \mathbf{Z}$, we write $n \cdot \Sigma=\sum_{\sigma \in \Sigma} n \sigma \in \mathbf{Z}[\Sigma]$. Let

$$
I^{+}=(1 \cdot \Sigma, \ldots, 1 \cdot \Sigma ; 0, \ldots, 0) \text { and } I^{-}=(0, \ldots, 0 ; 1 \cdot \Sigma, \ldots, 1 \cdot \Sigma) .
$$

Define the algebraic representation of $H(R)=\prod_{\sigma \in \Sigma} \mathrm{GL}_{r}(R) \times \mathrm{GL}_{s}(R)$ of the minimal weight $-\underline{k}$ by

$$
L_{\underline{k}}(R)=\bigotimes_{\sigma \in \Sigma} L_{k_{\sigma}}(R) \quad\left(\underline{k}=\sum k_{\sigma} \sigma\right) .
$$


We define the distinguished functional $l_{\underline{k}}$ :

$$
\begin{aligned}
l_{\underline{k}}: L_{\underline{k}}(R) & \rightarrow R \\
f & \rightarrow f(1) .
\end{aligned}
$$

Then $l_{\underline{k}}$ is the vector of maximal weight $\underline{k}$ in $L_{k}^{*}(R):=\operatorname{Hom}\left(L_{\underline{k}}(R), R\right)$. The $R$ module $L_{\underline{k}}(R)$ has a functorial decomposition into weight spaces of $T(R)$ :

$$
L_{\underline{k}}(R)=\bigoplus_{\chi \geq-\underline{k}} R v_{\chi}
$$

where $v_{\chi}$ is of weight $\chi$. Denote by $v_{-\underline{k}}$ the vector of the minimal weight $-\underline{k}$ in $L_{\underline{k}}(R)$ such that $l_{\underline{k}}\left(v_{-\underline{k}}\right)=1$.

Remark 3.1 (Twisted action). Let $X_{*}(T)$ be the co-character group of $T_{/ \mathcal{O}}$. Let $\mu \in X_{*}(T)$ such that $\langle\mu, \chi\rangle \leq 0$ for all positive roots $\chi$ (with respect to the unipotent subgroup $\left.N_{H}\right)$ and let $\alpha=\mu(p)$. We define the twisted action $\widetilde{\rho}_{\underline{k}}\left(\alpha^{-1}\right)$ on $L_{\underline{k}}(R)$ by

$$
\widetilde{\rho}_{\underline{k}}\left(\alpha^{-1}\right) v_{\chi}=p^{-\langle\mu, \underline{k}+\chi\rangle} v_{\chi} .
$$

Note that this action preserves $p$-integrality since $\chi \geq-\underline{k}$. In addition, if $p$ is invertible in $R$, we have

$$
\widetilde{\rho}_{\underline{k}}\left(\alpha^{-1}\right) v_{\chi}=\alpha^{-\underline{k}-\chi} v_{\chi}=\alpha^{-\underline{k}} \rho_{\underline{k}}\left(\alpha^{-1}\right) v_{\chi} .
$$

3.2. Geometric modular forms. We recall the theory of classical modular forms and $p$-adic modular forms on unitary groups from Katz-Hida's point of view. Recall that $G=G U(V)$ and $\underline{\omega}=e^{*} \Omega_{\mathcal{G} / \bar{S}_{G}(K)_{/ \mathcal{O}}}$. Decompose $\underline{\omega}=\oplus_{\sigma \in \mathrm{I}_{\mathcal{K}}} e_{\sigma} \underline{\omega}$ and define

$$
\mathcal{E}_{V}^{+}:=\bigoplus_{\sigma \in \Sigma} \underline{\operatorname{Isom}}\left(\mathcal{O}_{\bar{S}_{G}(K)}^{r}, e_{\sigma} \underline{\underline{\omega}}\right) ; \quad \mathcal{E}_{V}^{-}:=\bigoplus_{\sigma \in \Sigma^{c}} \underline{\operatorname{Isom}}\left(\mathcal{O}_{\bar{S}_{G}(K)}^{s}, e_{\sigma} \underline{\omega}\right) .
$$

Then $\mathcal{E}_{V}:=\mathcal{E}_{V}^{+} \oplus \mathcal{E}_{V}^{-}$is an $H$-torsor over $\bar{S}_{G}(K)$ with the structure map $\bar{\pi}: \mathcal{E}_{V} \rightarrow$ $\bar{S}_{G}(K)$. An $R$-point in $\mathcal{E}_{V}$ is a pair of $(x, \boldsymbol{\omega})$, where $x$ is an $R$-point in $\bar{S}_{G}(K)$ and $\boldsymbol{\omega}=\left(\boldsymbol{\omega}^{+}, \boldsymbol{\omega}^{-}\right): e^{+}\left(\mathcal{O}_{\mathcal{K}} \otimes R^{r}\right) \oplus e^{-}\left(\mathcal{O}_{\mathcal{K}} \otimes R^{s}\right) \simeq e^{+} \underline{\omega}_{x} \oplus e^{-} \underline{\omega}_{x}$ is an $\mathcal{O}_{\mathcal{K}} \otimes \mathbf{z} R$-module isomorphism. For $h=\left(h^{+}, h^{-}\right) \in H(R)=\operatorname{GL}_{r}\left(R^{\Sigma}\right) \times \mathrm{GL}_{s}\left(R^{\Sigma}\right), h$ acts on $\boldsymbol{\omega}$ by the rule $h \cdot \boldsymbol{\omega}\left(v^{+}, v^{-}\right)=\left(\boldsymbol{\omega}^{+}\left(v^{+} h^{+}\right), \boldsymbol{\omega}^{-}\left(v^{-} h^{-}\right)\right)$.

Definition 3.2 (Katz-Hida). Let $\underline{k}$ be a dominant weight such that $\|\underline{k}\|$ is parallel 1 Define the automorphic sheaf of weight $\underline{k}$ by

$$
\omega_{\underline{k}}:=\left(\bar{\pi}_{*} \mathcal{E}_{V}\right)^{N_{H}}\left[\underline{k}^{-1}\right] .
$$

Then $\omega_{\underline{k}}$ is a locally free coherent $\mathcal{O}_{\bar{S}_{G}(K)}$-module. A section $f$ of $\omega_{\underline{k}}$ can be regarded as a function on $\mathcal{E}_{V}$ such that

$$
f\left(x, t n_{+} \boldsymbol{\omega}\right)=\underline{k}\left(t^{-1}\right) f(x, \boldsymbol{\omega}) .
$$

Equivalently, we can define $\omega_{\underline{k}}=\mathcal{E}_{V} \times{ }^{H} L_{\underline{k}}$, and a section $f$ of $\omega_{\underline{k}}$ is a morphism $f: \mathcal{E}_{V} \rightarrow L_{\underline{k}}$ such that

$$
f(x, h \boldsymbol{\omega})=\rho_{\underline{k}}(h) f(x, \boldsymbol{\omega}) \text { for all } h \in H .
$$

\footnotetext{
${ }^{1}$ Because Aut $\underline{\mathcal{G}}=O_{+} \cap \nu(K) \neq\{1\}$, our moduli problem for $G$ is not rigid. We have to assume $\|\underline{k}\|$ is parallel to get a well-defined $\omega_{\underline{k}}$.
} 
Definition 3.3. Let $R$ be a base ring flat over $\mathbf{Z}_{(p)}$. Define the space of geometric modular forms over $R$ of weight $\underline{k}$ and level $K_{\bullet}^{n}$ by

$$
\mathbf{M}_{\underline{k}}\left(K_{\bullet}^{n}, R\right):=H^{0}\left(I_{G}\left(K_{\bullet}^{n}\right)_{/ R}, \omega_{\underline{k}}\right) \quad(\bullet=0,1, \emptyset) .
$$

If $R$ is a field of characteristic zero and $r s>1($ or $\mathcal{F} \neq \mathbf{Q})$, then it follows from the Koecher principle ( $c f$. [Shi97, A4.5, page 230]) that

$$
\mathbf{M}_{\underline{k}}\left(K_{\bullet}^{n}, R\right)=H^{0}\left(\bar{S}_{G}\left(K_{\bullet}^{n}\right)_{/ R}, \omega_{\underline{k}}\right) .
$$

3.3. $p$-adic modular forms. Let $\bar{H}_{p-1} \in H^{0}\left(S_{G}(K)_{/ \overline{\mathbb{F}}_{p}}, \operatorname{det}(\underline{\omega})^{p-1}\right)$ be the Hasse invariant. Since $\pi_{*} \operatorname{det} \omega$ is ample, we can lift a power of the Hasse invariant $\bar{H}_{p-1}^{t}$ for some sufficiently large positive integer $t$ to obtain a global section $E \in$ $H^{0}\left(S_{G}(K), \operatorname{det}(\underline{\omega})^{t(p-1)}\right)$, and by the Koecher principle, we have $E \in H^{0}\left(\bar{S}_{G}(K)\right.$, $\left.\operatorname{det}(\underline{\omega})^{t(p-1)}\right)$. By our notation, $\operatorname{det}(\underline{\omega})^{t(p-1)}=\omega_{k_{E}}$ is an automorphic sheaf of weight $k_{E}=t(p-1)\left(I^{+}+I^{-}\right)$. Let $m$ be a positive integer. We let $T_{0, m}:=$ $\bar{S}_{G}(K)[1 / E]_{/ \mathcal{O}_{m}}$ be the ordinary locus of $\bar{S}_{G}(K) / \mathcal{O}_{m}$. Moreover, it is known that under the ordinary condition (ord), $T_{0, m}$ is open and dense in $\bar{S}_{G}(K) / \mathcal{O}_{m}$ (Wed99]).

Let $V_{\underline{k}}\left(K, \mathcal{O}_{m}\right):=H^{0}\left(T_{0, m}, \omega_{\underline{k}}\right)$. By definition, we have

$$
V_{\underline{k}}\left(K, \mathcal{O}_{m}\right)=\underset{\lim }{\longrightarrow} \frac{H^{0}\left(\bar{S}_{G}(K) / \mathcal{O}_{m}, \omega_{\underline{k}+n k_{E}}\right)}{E^{n}} .
$$

Set $T_{n, m}:=I_{G}\left(K^{n}\right)_{/ \mathcal{O}_{m}}$ for $n>1$. Then $\pi_{n^{\prime}, m}: T_{n^{\prime}, m} \rightarrow T_{n, m}, n^{\prime} \geq n \geq 0$ is a finite étale. The Igusa tower $T_{\infty, m}$ over $\mathcal{O}_{m}$ is defined by

$$
T_{\infty, m}:={\underset{n}{n}}_{\lim _{n}} T_{n, m} .
$$

Then $T_{\infty, m}$ is a Galois cover of $T_{0, m}$ with Galois group $\mathbf{H}=\mathrm{GL}_{\mathcal{O}_{\mathcal{K}}}\left(M^{0}\right)$. Henceforth, we identify $\mathbf{H}$ (resp. $\mathbf{N}$ ) with the group $H\left(\mathbf{Z}_{p}\right)$ (resp. $N_{H}^{t}\left(\mathbf{Z}_{p}\right)$ ) with respect to the $p$-adic basis fixed in (1.4).

Definition 3.4. Let $R$ be a $p$-adic $\mathcal{O}_{\mathfrak{p}}$-algebra and let $R_{m}:=R / p^{m} R$ for $m \in \mathbf{Z}_{+}$. Define

$$
\begin{aligned}
V_{n, m} & =H^{0}\left(T_{n, m}, \mathcal{O}_{T_{n, m}}\right), \\
V_{\underline{k}}\left(K_{1}^{n}, R_{m}\right) & =H^{0}\left(T_{n, m / R_{m}}, \omega_{\underline{k}}\right)^{\mathbf{N}} .
\end{aligned}
$$

Let $V_{\infty, m}=\lim _{p n} V_{n, m}$ and let $V_{\infty, \infty}=\lim _{m} V_{\infty, m}$. We call $V_{p}(G, K):=V_{\infty, \infty}^{\mathbf{N}}$ the space of $p$-adic modular forms. Let $\mathbf{T}=T\left(\mathbf{Z}_{p}\right) \subset \mathbf{H}$ and let $\Lambda_{\mathbf{T}}:=\mathcal{O}_{\mathfrak{p}} \llbracket \mathbf{T} \rrbracket$. The Galois action of $\mathbf{T}$ on $V_{\infty, m}^{\mathbf{N}}$ is given by $[t] . f(\underline{A}, j)=f(\underline{A}, t j)$ for $t \in \mathbf{T}$ and $f \in V_{\infty, m}^{\mathbf{N}}$. This action makes $V_{\infty, m}^{\mathbf{N}}$ a discrete $\Lambda_{\mathbf{T}}$-module.

Suppose that $n \geq m$. In $\$ 2.3 .2$, to each $R_{m}$-quintuple $(\underline{A}, j)$ of level $K^{n}$ we can attach a canonical basis $\boldsymbol{\omega}(j)$ of $H^{0}\left(A, \Omega_{A}\right)$. In other words, the morphism $T_{n, m} \rightarrow \mathcal{E}_{V / \mathcal{O}_{m}},(\underline{A}, j) \mapsto(\underline{A}, j, \boldsymbol{\omega}(j))$ defines a section of $\mathcal{E}_{V}$ over $\mathcal{O}_{m}$ and induces the canonical isomorphism:

$$
\begin{aligned}
H^{0}\left(T_{n, m / R_{m}}, \omega_{\underline{k}}\right) & \stackrel{\sim}{\rightarrow} V_{n, m} \otimes_{\mathcal{O}} L_{\underline{k}}\left(R_{m}\right) \\
f & \mapsto \widehat{f}(\underline{A}, j)=f(\underline{A}, j, \boldsymbol{\omega}(j)) .
\end{aligned}
$$


M.-L. HSIEH

We shall call $\widehat{f}$ the $p$-adic avatar of $f$. Define the evaluation morphism

$$
\begin{aligned}
\boldsymbol{\beta}_{\underline{k}}: V_{\underline{k}}\left(K_{1}^{n}, \mathcal{O}_{m}\right) & \rightarrow V_{n, m}^{\mathbf{N}} \\
f & \mapsto \boldsymbol{\beta}_{\underline{k}}(f):=l_{\underline{k}}(\widehat{f}) .
\end{aligned}
$$

It follows from the definition that $\boldsymbol{\beta}_{k}$ is $\mathbf{T}$-equivariant in the sense that

$$
\boldsymbol{\beta}_{\underline{k}}([t] . f)=t^{-\underline{k}} \cdot[t] \cdot \boldsymbol{\beta}_{\underline{k}}(f) \text { for all } t \in T\left(\mathbf{Z}_{p}\right) .
$$

Proposition 3.5. Let $\mathbf{H}_{n}=\left\{h \in \mathbf{H} \mid h \equiv 1\left(\bmod p^{n} M^{0}\right)\right\}$ and let $J \supset \mathbf{H}_{n}$ be a subgroup of $\mathbf{H}$. Then we have

$$
H^{0}\left(T_{n, m / R_{m}}, \omega_{\underline{k}}\right)^{J}=\left\{f \in V_{n, m} \otimes_{\mathcal{O}} L_{\underline{k}}\left(R_{m}\right) \mid \rho_{\underline{k}}\left(h^{t}\right) \widehat{f}(\underline{A}, h j)=\widehat{f}(\underline{A}, j), h \in J\right\} .
$$

Proof. Let $f \in H^{0}\left(T_{n, m / R_{m}}, \omega_{\underline{k}}\right)$. Then $f$ belongs to $H^{0}\left(T_{n, m / R_{m}}, \omega_{\underline{k}}\right)^{J}$ if and only if for all $h \in J$, we have

$$
\begin{aligned}
f(\underline{A}, h j, \boldsymbol{\omega}(j))=f(\underline{A}, j, \boldsymbol{\omega}(j)) & \Longleftrightarrow f\left(\underline{A}, h j, h^{t} \boldsymbol{\omega}(h j)\right)=\widehat{f}(\underline{A}, j) \quad\left(\boldsymbol{\omega}(h j)=h^{-t} \boldsymbol{\omega}(j)\right) \\
& \Longleftrightarrow \rho_{\underline{k}}\left(h^{t}\right) \widehat{f}(\underline{A}, h j)=\widehat{f}(\underline{A}, j) .
\end{aligned}
$$

3.4. Complex modular forms and automorphic forms. Define the standard cocycle $J: \mathrm{R}_{\mathcal{F} / \mathbf{Q}} G(\mathbf{R})^{+} \times X^{+} \rightarrow \mathrm{GL}_{r}\left(\mathbf{C}^{\Sigma}\right) \times \mathrm{GL}_{s}\left(\mathbf{C}^{\Sigma}\right)=H(\mathbf{C})$ by

$$
\alpha p(\tau)=p(\alpha \tau) J(\alpha, \tau), \forall(\alpha, \tau) \in G(\mathbf{R})^{+} \times X^{+} .
$$

We recall the explicit formula for $J(\alpha, \tau)=(\kappa(\alpha, \tau), \mu(\alpha, \tau))$ when $r s>0$ Shi97, (6.3.6) and (6.3.7)]. Writing $\alpha \in \mathrm{R}_{\mathcal{F} / \mathbf{Q}} G(\mathbf{R})^{+}$in the matrix form as in (2.3) and $\tau=\left[\begin{array}{l}x \\ y\end{array}\right]$, we have

$$
\kappa(\alpha, \tau)=\left[\begin{array}{cc}
\bar{h} x^{t}+\bar{d} & \bar{h} y^{t}+\bar{l} \bar{\vartheta} \\
-\bar{\vartheta}^{-1}\left(\bar{g} x^{t}+\bar{f}\right) & -\bar{\vartheta}^{-1} \bar{g} y^{t}+\bar{\vartheta}-1 \bar{e} \bar{\vartheta}
\end{array}\right] ; \mu(\alpha, \tau)=h x+l y+d
$$

If $s=0$ and $X^{+}=\left\{\mathbf{x}_{0}\right\}$, we put $J\left(\alpha, \mathbf{x}_{0}\right)=\nu(\alpha) \alpha^{-t} \in \mathrm{GL}_{r}\left(\mathbf{C}^{\Sigma}\right)$. Fix a base point $\mathbf{i} \in X^{+}$and let $K_{\infty}^{0}$ be the stabilizer of $\mathbf{i}$ in $\mathrm{R}_{\mathcal{F} / \mathbf{Q}} G(\mathbf{R})$. Then $J: K_{\infty}^{0} \longrightarrow$ $H(\mathbf{C}), k_{\infty} \mapsto J\left(k_{\infty}, \mathbf{i}\right)$ defines an algebraic representation of $K_{\infty}^{0}$.

We define the algebraic representation $L^{\underline{k}}(\mathbf{C})$ of $H(\mathbf{C})$ with the highest weight $\underline{k}$ as follows. The underlying space of $L^{\underline{k}}(\mathbf{C})$ is $L_{\underline{k}}(\mathbf{C})$ and the group action is given by

$$
\rho^{\underline{k}}(h)=\rho_{\underline{k}}\left(h^{-t}\right), h \in H(\mathbf{C}) .
$$

Definition 3.6. Let $U \subset G\left(\mathbf{A}_{\mathcal{F}, f}\right)$ be an open-compact subgroup. A holomorphic function $f: X^{+} \times G\left(\mathbf{A}_{\mathcal{F}, f}\right) \rightarrow L_{\underline{k}}(\mathbf{C})$ is called a holomorphic modular form of weight $\underline{k}$ and level $U$ if

$$
f(\alpha \tau, \alpha g u)=\nu(\alpha)^{-\|\underline{k}\|} \rho^{\underline{k}}(J(\alpha, \tau)) f(\tau, g) \text { for all }(\alpha, u) \in G(\mathcal{F})^{+} \times U .
$$

We denote by $\mathbf{M}_{\underline{k}}^{\mathrm{an}}(U, \mathbf{C})$ the space of holomorphic modular forms of weight $\underline{k}$ and level $U$.

The following lemma is a consequence of the GAGA principle, which enables us to identify geometric modular forms over $\mathbf{C}$ with holomorphic modular forms. 
Lemma 3.7. Let $d \underline{z}_{V}=\left(d \underline{z}_{V}(\Sigma), d \underline{z}_{V}\left(\Sigma^{c}\right)\right)$ be the $\mathcal{O}_{\mathcal{K}} \otimes_{\mathbf{Z}} \mathbf{C}$-basis of the space of invariant one forms of $\mathcal{A}(V)$ defined as in (2.10). Then we have the isomorphism:

$$
\begin{aligned}
H^{0}\left(S_{G}(U)_{/ \mathbf{C}}, \omega_{\underline{k}}\right)=\mathbf{M}_{\underline{k}}(U, \mathbf{C}) & \stackrel{\sim}{\rightarrow} \mathbf{M}_{\underline{k}}^{\mathrm{an}}(U, \mathbf{C}), \\
f & \mapsto f(\tau, g):=f\left(\mathcal{A}(V)_{g}(\tau), \overline{\left.\langle,\rangle_{c a n}, \iota_{\mathbf{C}}, \eta_{g} U, 2 \pi i d \underline{z}_{V}\right) .}\right.
\end{aligned}
$$

For each weight $\underline{k}=\left(a_{1}, \ldots a_{r} ; b_{s}, \ldots, b_{1}\right)$, we define $|\underline{k}| \in \mathbf{Z}\left[\mathrm{I}_{\mathcal{K}}\right]$ by

$$
|\underline{k}|=\sum_{\sigma \in \Sigma}\left(b_{1, \sigma}+\cdots+b_{s, \sigma}\right) \cdot \sigma+\left(a_{1, \sigma}+\cdots+a_{r, \sigma}\right) \cdot \sigma c .
$$

Definition 3.8. Let $\chi$ be a Hecke character of $\mathcal{K}^{\times}$with infinity type $|\underline{k}|$. Let $\mathcal{A}_{\underline{k}}(G, U, \chi)$ be the space of automorphic forms of weight $\underline{k}$ and level $U$ with central character $\chi$. In other words, $\mathcal{A}_{\underline{k}}(G, U, \chi)$ consists of smooth and slowly increasing functions $F: G\left(\mathbf{A}_{\mathcal{F}}\right) \rightarrow L_{\underline{k}}(\mathbf{C})$ such that for every $\left(\alpha, k_{\infty}, u, z\right) \in$ $G(\mathcal{F}) \times K_{\infty}^{0} \times U \times Z\left(\mathbf{A}_{\mathcal{F}}\right)$, we have

$$
F\left(z \alpha g k_{\infty} u\right)=\rho^{\underline{k}}\left(J\left(k_{\infty}, \mathbf{i}\right)^{-1}\right) F(g) \chi^{-1}(z) .
$$

We associate a $L_{\underline{k}}(\mathbf{C})$-valued function $\underline{\operatorname{AM}}(F)$ on $X^{+} \times G\left(\mathbf{A}_{\mathcal{F}, f}\right)$ to a function $F \in \mathcal{A}_{k}(G, U, \chi)$ defined by

$$
\underline{\operatorname{AM}}(F)(\tau, g):=\chi_{f}(\nu(g)) \rho^{\underline{k}}\left(J\left(g_{\infty}, \mathbf{i}\right)\right) F\left(\left(g_{\infty}, g\right)\right),
$$

where $g_{\infty} \in \mathrm{R}_{\mathcal{F} / \mathbf{Q}} G(\mathbf{R})^{+}$such that $g_{\infty} \mathbf{i}=\tau$. Then $\underline{\operatorname{AM}}(F)$ is a well-defined $L_{\underline{k}}(\mathbf{C})$ valued function on $X^{+} \times G\left(\mathbf{A}_{\mathcal{F}, f}\right) / U$. We put

$$
\mathcal{A}_{\underline{k}}^{\text {hol }}(G, U, \chi)=\left\{F \in \mathcal{A}_{\underline{k}}(G, U, \chi) \mid \underline{\operatorname{AM}}(F) \text { is holomorphic on } X^{+}\right\} .
$$

It is easy to see that $\underline{\mathrm{AM}}$ induces an injective map

$$
\underline{\mathrm{AM}}: \mathcal{A}_{\underline{k}}^{h o l}(G, U, \chi) \hookrightarrow \mathbf{M}_{\underline{k}}^{\mathrm{an}}(U, \mathbf{C}) .
$$

3.5. Modular forms on totally definite unitary groups. Suppose that $G=$ $G U(W)$ is a totally definite unitary group (i.e. $s=0$ ). We give a simple description of complex and $p$-adic modular forms on $G$. Let $U \subset G\left(\mathbf{A}_{\mathcal{F}, f}\right)$ be an open compact subgroup. If $R$ is a base ring such that $R=R[1 / p]$, then the space $\mathbf{M}_{\underline{k}}(U, R)$ of modular forms over $R$ is the space consisting of functions $f: G\left(\mathbf{A}_{\mathcal{F}, f}\right) \rightarrow L_{\underline{k}}(R)$ such that

$$
f(\alpha g u)=\rho_{\underline{k}}(\alpha) f(g) \text { for all }(\alpha, u) \in G(\mathcal{F}) \times U .
$$

On the other hand, suppose that $R$ is a $p$-adic $\mathcal{O}_{\mathfrak{p}}$-algebra. Let $\widehat{\mathbf{M}}_{\underline{k}}(U, R)$ be the space of functions $f: G\left(\mathbf{A}_{\mathcal{F}, f}\right) \rightarrow L_{\underline{k}}(R)$ such that

$$
f(\alpha g u)=\rho_{\underline{k}}\left(u_{p}^{-1}\right) f_{p}(g) \text { for all } \alpha \in G(\mathcal{F}), u \in U .
$$

Then $\widehat{\mathbf{M}}_{\underline{k}}\left(K_{1}^{n}, R_{m}\right)=V_{\underline{k}}\left(K_{1}^{n}, R_{m}\right)$. In addition, if $R=R[1 / p]$, then we have a natural isomorphism

$$
\begin{aligned}
\mathbf{M}_{\underline{k}}(U, R) & \stackrel{\sim}{\rightarrow} \widehat{\mathbf{M}}_{\underline{k}}(U, R) \\
f & \mapsto \widehat{f}, \widehat{f}(g):=\rho_{\underline{k}}\left(g_{p}^{-1}\right) f(g) .
\end{aligned}
$$

Proposition 3.9 (Base change). If $U$ satisfies (neat), then $\widehat{\mathbf{M}}_{\underline{k}}\left(U, \mathcal{O}_{\mathfrak{p}}\right) \otimes_{\mathcal{O}_{\mathfrak{p}}} R=$ $\widehat{\mathbf{M}}_{k}(U, R)$. 
Proof. We write

$$
G\left(\mathbf{A}_{\mathcal{F}, f}\right)=\bigsqcup_{i \in X} G(\mathcal{F}) t_{i} U
$$

for a finite set $X$. The assumption (neat) implies that $U \cap t_{i}^{-1} G\left(\mathbf{A}_{\mathcal{F}, f}\right) t_{i}=U \cap O_{+}^{\times}$. In addition, $\rho_{\underline{k}}\left(O_{+}^{\times}\right)$acts trivially since $\|\underline{k}\|$ is parallel. We thus find that for every $\mathcal{O}_{\mathfrak{p}}$-algebra $R$,

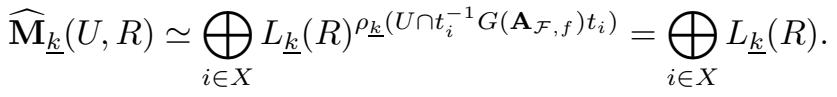

The proposition follows immediately.

3.6. Fourier-Jacobi expansion and Siegel operators. In this subsection, we assume that $s=1$ (so $G=G U(r, 1)$ ) and retain the notation in 2.7 We give a brief account of Fourier-Jacobi expansion of modular forms on $G$.

3.6.1. To begin with, we introduce some notation for the totally definite unitary group $G_{P}=G U(W)$. Let $H_{P}:=\mathrm{R}_{O / \mathbf{z}} \mathrm{GL}_{r-1}$. Let $T_{P}:=\mathrm{R}_{O / \mathbf{z}} T_{r-1}$ be the diagonal torus and let $U_{P}:=\mathrm{R}_{O / \mathbf{z}} N_{r-1}$ be the upper triangular unipotent subgroup of $H_{P}$. We shall regard $H_{P}$ as a subgroup of $H$ by $x \mapsto \operatorname{diag}(1, x, 1)$. Let $I_{[g]}^{n}:=$ $I_{[g]}\left(K_{1}^{n}\right)$ for $g \in G\left(\mathbf{A}_{\mathcal{F}, f}^{(p)}\right)$. Recall that $A_{[g]}^{n}=H^{0}\left(I_{[g]}^{n}, \mathcal{O}_{I_{[g]}^{n}}\right)$ is the ring of regular functions on $I_{[g]}^{n}$. Then $A_{[g]}^{n}$ is nothing but the space of $\mathcal{O}$-valued functions on $G_{P}(\mathcal{F}) \backslash G_{P}\left(\mathbf{A}_{\mathcal{F}, f}\right) / K_{P, 1}^{g, n}$. The space $V_{[g]}$ of $p$-adic modular forms for $\left(G_{P}, K_{P}^{g}\right)$ is given by

$$
V_{[g]}:=\underset{n}{\lim _{n}} A_{[g]}^{n} \otimes_{\mathcal{O}} \mathcal{O}_{\mathfrak{p}}=\bigcup_{n}\left\{f: G_{P}(\mathcal{F}) \backslash G_{P}\left(\mathbf{A}_{\mathcal{F}, f}\right) / K_{P, 1}^{n, g} \rightarrow \mathcal{O}_{\mathfrak{p}}\right\} .
$$

Let $\mathbf{T}_{P}:=T_{P}\left(\mathbf{Z}_{p}\right)$ and $\Lambda_{P}:=\mathcal{O}_{\mathfrak{p}} \llbracket \mathbf{T}_{P} \rrbracket$. The action of $\mathbf{T}_{P}$ on $V_{[g]}$ is given by $[t] . f\left(g_{1}\right)=f\left(g_{1} t\right)$, where $t \in \mathbf{T}_{P} \subset U(W)\left(\mathcal{F}_{p}\right)$. This makes $V_{[g]}$ a compact $\Lambda_{P^{-}}$ module.

3.6.2. Fourier-Jacobi expansion. Each pair $(g, h) \in C(K) \times \mathbf{H}$ can be regarded as a $p$-adic cusp, i.e. cusps of the Igusa tower. Suppose that $(g, h)$ is a $p$-adic cusp such that $h^{-1} U_{P}\left(\mathbf{Z}_{p}\right) h \subset \mathbf{N}$. Let $\underline{\mathcal{B}}$ be the universal family over the Shimura variety $S_{[g]}$ associated to $G_{P}$ with the structure morphism $\pi: \underline{\mathcal{B}} \rightarrow S_{[g]}$. The exact sequence (2.20) induces the exact sequence of $\mathcal{O}_{\mathcal{K}}$-modules

$$
0 \longrightarrow \Omega_{\mathcal{B}} \longrightarrow \Omega_{\mathcal{M}} \longrightarrow \Omega_{\mathcal{T}_{g}} \longrightarrow 0, \mathcal{T}_{g}:=X_{g}^{\vee} \otimes \mathbb{G}_{m} .
$$

Let $\boldsymbol{\omega}_{\mathcal{B}}$ be the $\mathcal{O}_{\mathcal{K}} \otimes \mathbf{z} \mathcal{O}_{S_{[g]}}$-basis of $\pi_{*} \Omega_{\mathcal{B} / S_{[g]}}$ chosen in $\$ 2.8$ for the totally definite

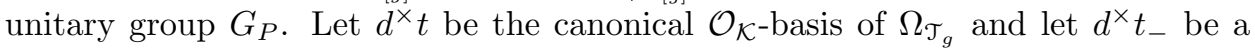
canonical lifting of $e^{-} \cdot d^{\times} t$ in $\Omega_{\mathcal{M}}$. Choose a lifting $d^{\times} t_{+}^{\prime}$ of $e^{+} \cdot d^{\times} t$ in $\Omega_{\mathcal{M}}$ and let $\boldsymbol{\omega}_{\mathcal{M}}:=\left(d^{\times} t_{+}^{\prime}, \boldsymbol{\omega}_{\mathcal{B}} ; d^{\times} t_{-}\right)$. Then $\left(\underline{\mathcal{M}}_{[g]}, \boldsymbol{\omega}_{\mathcal{M}}\right)$ gives rise to a point in $\mathcal{E}_{V}\left(\mathcal{R}_{[g], n}^{\xi}\right)$. Evaluating a modular form $f \in H^{0}\left(I_{G}\left(K_{1}^{n}\right)_{/ R}, \omega_{\underline{k}}\right)$ over an $\mathcal{O}$-algebra $R$ at $\left(\underline{\mathcal{M}}_{[g]}, h^{-1} j_{\mathcal{M}}\right.$, $\left.\boldsymbol{\omega}_{\mathcal{M}}\right)$, we obtain the Fourier-Jacobi expansion of $f$ at the cusp $(g, h)$ :

$$
F J_{[g]}^{h}(f):=f\left(\underline{\mathcal{M}}_{[g]}, h^{-1} j_{\mathcal{M}}, \boldsymbol{\omega}_{\mathcal{M}}\right) \in L_{\underline{k}}\left(\mathcal{R}_{[g], n}^{\xi}\right) \otimes_{\mathcal{O}} R .
$$

Let $N_{H}^{1}:=\left\{\left[\begin{array}{cc}1 & 0 \\ * & 1_{r-1}\end{array}\right]\right\} \times\{1\} \subset H$. Note that the $N_{H}^{1}$-orbit $N_{H}^{1} \boldsymbol{\omega}_{\mathcal{M}}$ does not depend on the choice of the lifting $d^{\times} t_{+}^{\prime}$. Therefore, the image $F J_{[g]}^{h}(f)_{N_{H}^{1}}$ of 
$F J_{[g]}^{h}(f)$ in the $N_{H}^{1}$-covariant module $L_{\underline{k}}\left(\mathcal{R}_{[g], n}^{\xi}\right)_{N_{H}^{1}} \otimes_{\mathcal{O}} R$ only depends on the choice of $\omega_{\mathcal{B}}$. We thus obtain the expansion

$$
\begin{aligned}
F J_{[g]}^{h}(f)_{N_{H}^{1}} & =\sum_{\beta \in \mathscr{S}_{[g]}^{0}} \mathbf{a}_{[g]}^{h}(\beta, f) q^{\beta}, \text { where } \\
& \mathbf{a}_{[g]}^{h}(\beta, f) \in L_{\underline{k}}\left(A_{[g]}^{n} \otimes_{\mathcal{O}} R\right)_{N_{H}^{1}} \otimes_{A_{[g]}} H^{0}\left(\mathcal{Z}_{[g]}^{\circ}, \mathcal{L}(\beta)\right) .
\end{aligned}
$$

We consider the Fourier-Jacobi expansions of $p$-adic modular forms. Let $\mathcal{R}_{[g], \infty}:=$ $\lim _{\longrightarrow n} \mathcal{R}_{[g], n} \otimes_{\mathcal{O}} \mathcal{O}_{\mathfrak{p}}$. If $f \in V_{\infty, m}^{\mathbf{N}}$, then the $p$-adic Fourier-Jacobi expansion at the cusp $(g, h)$ is defined by

$$
\widehat{F J}_{[g]}^{h}(f):=f\left(\underline{\mathcal{M}}_{[g]}, h^{-1} j_{\mathcal{M}}\right)=\sum_{\beta \in \mathscr{S}_{[g]}^{0}} \widehat{\mathbf{a}}_{[g]}^{h}(\beta, f) q^{\beta} \in \mathcal{R}_{[g], \infty} \otimes_{\mathcal{O}_{\mathfrak{p}}} \mathcal{O}_{m} .
$$

If $f \in V_{\underline{k}}\left(K_{1}^{n}, \mathcal{O}_{m}\right)$, then we define $\widehat{F J}_{[g]}^{h}(f):=\widehat{F J}_{[g]}^{h}\left(\boldsymbol{\beta}_{\underline{k}}(f)\right)$.

3.6.3. Siegel $\Phi$-operators. Define the Siegel $\Phi$-operator by

$$
\begin{aligned}
\Phi_{[g]}^{h}: H^{0}\left(I_{G}\left(K_{1}^{n}\right) / R, \omega_{\underline{k}}\right) & \longrightarrow L_{\underline{k}}\left(A_{[g]}^{n} \otimes_{\mathcal{O}} R\right)_{N_{H}^{1}} \\
f & \mapsto \Phi_{[g]}^{h}(f):=\mathbf{a}_{[g]}^{h}(0, f)_{N_{H}^{1}} .
\end{aligned}
$$

Remark 3.10. To each weight $\underline{k}=\left(a_{1}, \ldots a_{r} ; b_{1}\right)$ of $T$, we associate a weight $\underline{k}^{\prime}:=\left(a_{1}, a_{2}, \ldots, a_{r-1}\right)$ of $T_{P}$. Then $-\underline{k}^{\prime}$ is the minimal weight of the representation $\left.\left(L_{\underline{k}}\right)_{N_{H}^{1}}\right|_{H_{P}}$ over a field. It follows that if $R$ is a field and $f \in \mathbf{M}_{\underline{k}}\left(K_{1}^{n}, R\right)$, then $\mathbf{a}_{[g]}^{h}(0, f)$ belongs to $L_{\underline{k}^{\prime}}\left(A_{[g]}^{n} \otimes_{\mathcal{O}} R\right)$.

The $p$-adic Siegel $\Phi$-operator $\widehat{\Phi}_{[g]}^{h}: V_{\infty, m}^{\mathbf{N}} \rightarrow V_{[g]} \otimes_{\mathcal{O}} \mathcal{O}_{m}$ is defined by

$$
f \mapsto \widehat{\Phi}_{[g]}^{h}(f)=\widehat{\mathbf{a}}_{[g]}^{h}(0, f) .
$$

When $h=1$, we drop the superscript and simply write $F J_{[g]}$ (resp. $\left.\Phi_{[g]}\right)$ for $F J_{[g]}^{1}$ $\left(\right.$ resp. $\left.\Phi_{[g]}^{1}\right)$. Let

$$
w_{r}:=\operatorname{diag}\left(\left[\begin{array}{cc}
0 & 1 \\
\mathbf{1}_{r-1} & 0
\end{array}\right], 1\right) \in H_{P}\left(\mathbf{Z}_{p}\right) .
$$

We have the following commutative diagram:

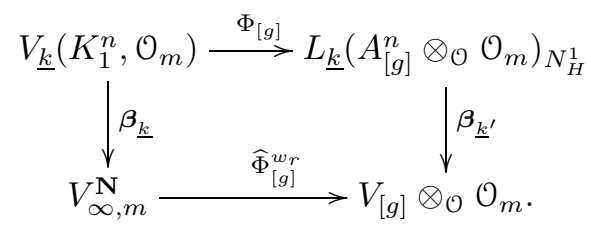

3.6.4. The q-expansion principle. We put

$$
\mathcal{R}_{[g]}^{\mathbf{T}}=\prod_{h \in \mathbf{T}} \mathcal{R}_{[g], \infty} \text { and } \mathcal{R}_{C}^{\mathbf{T}}=\prod_{g \in C(K)} \mathcal{R}_{[g]}^{\mathbf{T}} .
$$

Define the Fourier-Jacobi map:

$$
\begin{aligned}
\widehat{F J}: V_{\infty, m}^{\mathbf{N}} & \longrightarrow \mathcal{R}_{C}^{\mathbf{T}} \otimes_{\mathcal{O}_{\mathfrak{p}}} \mathcal{O}_{m} \\
f & \mapsto \widehat{F J}(f)=\left(\widehat{F J}_{[g]}^{h}(f)\right)_{(g, h) \in C(K) \times \mathbf{T}} .
\end{aligned}
$$


Thanks to the work of Ching-Li Chai and Hida (see Cha08 for the case $U(n, n)$ and Hid09a] for general unitary groups), it is known that the image of the monodromy group of the ordinary locus $\left(S_{G}(K) \otimes \overline{\mathbb{F}}_{p}\right)^{\text {ord }}$ in $\operatorname{Aut}_{\mathcal{O}_{\mathcal{K}}}\left(\mathcal{A}^{\text {ord }}\left[p^{\infty}\right]\right)$ is isomorphic to $\left(\mathrm{GL}_{r}\left(O_{p}\right) \times \mathrm{GL}_{s}\left(O_{p}\right)\right)^{\mathrm{det}=1}$. In particular, this implies that $C(K) \times \mathbf{T}$ contains at least one cusp at each connected component of $I_{G}\left(K_{\bullet}^{n}\right) \otimes \overline{\mathbb{F}}_{p}$ and hence the following $q$-expansion principle for $p$-adic modular forms.

Theorem 3.11 (The $q$-expansion principle). The Fourier-Jacobi expansion $\widehat{F J}$ : $V_{\infty, m}^{\mathbf{N}} \rightarrow \mathcal{R}_{C}^{\mathbf{T}} \otimes_{\mathcal{O}_{\mathfrak{p}}} \mathcal{O}_{m}$ is injective.

3.6.5. Analytic Fourier-Jacobi expansions. Let $U \subset G\left(\mathbf{A}_{\mathcal{F}, f}\right)$ be an open-compact subgroup and let $R \subset \mathbf{C}$ be a base ring. Let $f \in \mathbf{M}_{k}^{\text {an }}(U, \mathbf{R})=\mathbf{M}_{\underline{k}}(U, R)$. Then $f$ can be viewed as a function on $X_{r, 1} \times G\left(\mathbf{A}_{\mathcal{F}, f}\right)$. We have the Fourier-Jacobi expansion of $f$ at the standard maximal parabolic $P$ :

$$
\begin{aligned}
f\left(\left[\begin{array}{l}
x \\
y
\end{array}\right], g\right)= & \mathbf{a}_{0}(g, f)+\sum_{\beta \in \mathcal{F}_{+}} \mathbf{a}_{\beta}(y, g, f) \exp \left(2 \pi i \operatorname{Tr}_{\mathcal{F} / \mathbf{Q}}(\beta x)\right) \\
& \times\left(\left[\begin{array}{l}
x \\
y
\end{array}\right], g\right) \in X_{r, 1} \times G\left(\mathbf{A}_{\mathcal{F}, f}\right)
\end{aligned}
$$

Here the functions $\mathbf{a}_{\beta}(-, g, f):\left(\mathbf{C}^{\Sigma}\right)^{r-1} \rightarrow \mathbf{C}$ are theta functions with complex multiplication by $\mathcal{K}$. We put

$$
F J_{P}(g, f)=\mathbf{a}_{0}(g, f)+\sum_{\beta \in \mathcal{F}_{+}} \mathbf{a}_{\beta}(y, g, f) q^{\beta} .
$$

The formal power series $F J_{P}(g, f)$ is called the analytic Fourier-Jacobi expansion of $f$ at $g$.

Let $(g, h) \in C(K) \times \mathbf{H}$. We have seen that $I_{[g]}^{n}(\mathbf{C})=G_{P}(\mathcal{F}) \backslash G_{P}\left(\mathbf{A}_{\mathcal{F}, f}\right) / K_{P, 1}^{n, g}$ in 2.4. For each $g_{1} \in G_{P}\left(\mathbf{A}_{\mathcal{F}, f}\right)$ let $\left[g_{1}\right]$ be the class of $g_{1}$ in $I_{[g]}^{n}(\mathbf{C})$ and let $\varphi_{\left[g_{1}\right]}: A_{[g]}^{n} \rightarrow \mathbf{C}$ be the associated $\mathbf{C}$-algebra homomorphism. We have the following important comparison between analytic and algebraic Fourier-Jacobi expansions Lan12]:

$$
\begin{aligned}
F J_{P}\left(g_{1} h g, f\right)_{N_{H}^{1}(\mathbf{C})} & =\varphi_{\left[g_{1}\right]}\left(f\left(\underline{\mathcal{M}}_{[g]}, h^{-1} j_{\mathcal{M}},\left(d^{\times} t_{+}^{\prime}, 2 \pi i d \underline{z}_{W} ; d^{\times} t_{-}\right)\right)\right)_{N_{H}^{1}(\mathbf{C})} \\
& =\rho_{\underline{k}}\left(\operatorname{diag}\left(1,2 \pi i \Omega_{G_{P}, \infty}^{-1}, 1\right)\right) \varphi_{\left[g_{1}\right]}\left(F J_{[g]}^{h}(f)\right)_{N_{H}^{1}(\mathbf{C})},
\end{aligned}
$$

where we identify $\mathbf{H}$ with a subgroup of $U(V)\left(\mathcal{F}_{p}\right)$ by $h=\left(h_{+}, h_{-}\right) \in \mathrm{GL}_{r}\left(O_{p}\right) \times$ $\mathrm{GL}_{1}\left(O_{p}\right) \rightarrow \operatorname{diag}\left(h_{+}^{-1}, h_{-}\right) \in \mathrm{GL}_{r+s}\left(\mathcal{F}_{p}\right)=U(V)\left(\mathcal{F}_{p}\right)$.

3.6.6. Analytic Siegel operator. For $g \in G\left(\mathbf{A}_{\mathcal{F}, f}\right)$, the analytic Siegel operator $\Phi_{P, g}$ sends $f$ to a function $\Phi_{P, g}(f)$ on $G_{P}\left(\mathbf{A}_{\mathcal{F}, f}\right)$ defined by

$$
\Phi_{P, g}(f)\left(g_{1}\right):=\lim _{t \rightarrow+\infty} f\left(t \mathbf{i}_{0}, g_{1} g\right)=\mathbf{a}_{0}\left(g_{1} g, f\right) \quad\left(\mathbf{i}_{0}=\left(\left[\begin{array}{c}
\sqrt{-1} \\
0
\end{array}\right]\right)_{\sigma \in \Sigma} \in X_{r, 1}\right) .
$$


Then $\Phi_{P, g}(f)$ has the following integral expression:

$$
\Phi_{P, g}(f)\left(g_{1}\right)=\int_{N_{P}(\mathcal{F}) \backslash N_{P}\left(\mathbf{A}_{\mathcal{F}}\right)} f\left(n \cdot\left(\mathbf{i}_{0}, g_{1} g\right)\right) d n,
$$

where $d n$ is the normalized Haar measure of $N\left(\mathbf{A}_{\mathcal{F}}\right)$ so that $\operatorname{vol}(N(\mathcal{F}) \backslash$ $\left.N\left(\mathbf{A}_{\mathcal{F}}\right), d n\right)=1$.

Lemma 3.12. The function $\Phi_{P, g}(f)$ takes value in $L_{\underline{k}^{\prime}}(\mathbf{C})$. Moreover, $\Phi_{P, g}(f) \in$ $\mathbf{M}_{\underline{k}^{\prime}}\left(U^{g}, \mathbf{C}\right)$ is a modular form on $G_{P}$ of weight $\underline{k}^{\prime}$ (Recall that $\underline{k}^{\prime}=\left(a_{1}, \ldots, a_{r-1}\right)$ if $\left.\underline{\underline{k}}=\left(a_{1}, \ldots, a_{r} ; b_{1}\right)\right)$.

Proof. By [Har84, page $71(2.2 .2 .6)], \Phi_{P, g}(f)$ indeed takes value in $L^{\underline{k}}(\mathbf{C})^{N_{H}^{1}(\mathbf{C})}$. Viewing $L^{\underline{k}}(\mathbf{C})^{N_{H}^{1}(\mathbf{C})}$ as the representation of $M_{P}(\mathbf{C})=(\mathcal{K} \otimes \mathbf{C})^{\times} \times H_{P}(\mathbf{C}) \simeq$ $\mathbf{C}^{\times}(\Sigma) \times \mathbf{C}^{\times}\left(\Sigma^{c}\right) \times \mathrm{GL}_{r-1}(\mathbf{C}(\Sigma))$, we have

$$
\left.L^{\underline{k}}(\mathbf{C})^{N_{H}^{1}(\mathbf{C})}\right|_{M_{P}(\mathbf{C})}=\left.L_{\underline{k}}(\mathbf{C})_{N_{H}^{1}(\mathbf{C})}\right|_{M_{P}(\mathbf{C})}=L_{a_{r}}(\mathbf{C}) \otimes L_{b_{1}}(\mathbf{C}) \otimes L_{\underline{k^{\prime}}}(\mathbf{C})
$$

(cf. [Jan87, II, Prop. 2.11] and Remark 3.10). In particular, $\left.L^{\underline{k}}(\mathbf{C})^{N_{H}^{1}(\mathbf{C})}\right|_{G_{P}(\mathbf{C})}=$ $L_{\underline{k}^{\prime}}(\mathbf{C})$. It is straightforward to verify that $\Phi_{P, g}(f)$ belongs to $\mathbf{M}_{\underline{k}^{\prime}}\left(U^{g}, \mathbf{C}\right)$, using formulas in (3.5) and (3.6).

By [Har84, Prop. 2.4.4] and the above lemma, we obtain an exact sequence

$$
0 \rightarrow \mathbf{M}_{\underline{k}}^{0}(U, \mathbf{C}) \longrightarrow \mathbf{M}_{\underline{k}}(U, \mathbf{C}) \stackrel{\Phi_{P}=\oplus \Phi_{P, g}}{\longrightarrow} \bigoplus_{[g] \in \underline{C}(U)} \mathbf{M}_{\underline{k}^{\prime}}\left(U^{g}, \mathbf{C}\right),
$$

where $\mathbf{M}_{\underline{k}}^{0}(U, \mathbf{C})$ is the space of cusp forms of weight $\underline{k}$ and level $U$ and $\underline{C}(U)$ is the set of cusps of $S_{G}(U)(\mathbf{C})$.

If $U=K_{1}^{n}$ and $h \in \mathbf{H}$ with $h^{-1} U_{P}\left(\mathbf{Z}_{p}\right) h \subset \mathbf{N}$, then by (3.12) we have

$$
\Phi_{P, h g}(f)=\rho_{\underline{k}^{\prime}}\left(2 \pi i \Omega_{G_{P}, \infty}^{-1}\right) \Phi_{[g]}^{h}(f) \in \mathbf{M}_{\underline{k}^{\prime}}\left(K_{P, 1}^{g, n}, \mathbf{C}\right) .
$$

3.7. Hecke correspondence and Hecke operators. In this subsection, we review the interpretation of the action of Hecke operators as algebraic correspondences on the sheaf $\omega_{\underline{k}}$ over various base rings.

3.7.1. Hecke correspondence outside $p$. Let $\ell$ be a prime. For each non-negative integer $n$, let $\mathcal{O}_{\ell, n}=\mathcal{O}\left[e^{2 \pi i / p^{n}}, \frac{1}{\ell}\right]$. We consider the Hecke correspondence at $\ell$ for $I_{G}\left(K_{\bullet}^{n}\right) / \mathcal{O}_{\ell, n}$ with $\bullet=0,1$. We let $I_{G}\left(K_{\bullet}^{0}\right)=S_{G}(K)$ if $n=0$. Let $\alpha \in$ $\mathrm{R}_{\mathcal{F} / \mathbf{Q}} G\left(\mathbf{Q}_{\ell}\right) \cap \operatorname{End}\left(M \otimes \mathbf{Z}_{\ell}\right)$ and $\alpha^{\vee}$ be the dual of $\alpha$ under the Weil pairing induced by the polarization. Then $\alpha^{\vee}=\alpha^{-1} \nu(\alpha)$. An $\ell$-isogeny of type $\alpha$ is the isomorphism class of a triple $z=\left(x \stackrel{\varphi}{\rightarrow} x_{\alpha}\right)$, consisting of points $x=(\underline{A}, \bar{j})$ and $x_{\alpha}=\left(\underline{A}_{\alpha}, \bar{j}_{\alpha}\right)$ of $I_{G}\left(K_{\bullet}^{n}\right) / \mathcal{O}_{n, \ell}$ and an $\ell$-isogeny $\varphi$ such that

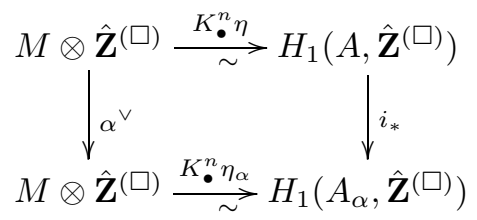

with $i^{*} \bar{\lambda}=\nu(\alpha) \bar{\lambda}_{\alpha}$. Here $\square=\emptyset$ if $\ell=p$ and $\square=\{p\}$ if $\ell \neq p$. 
Consider the scheme $Z_{\alpha}$ classifying $\ell$-isogeny of type $\alpha$ and the following correspondence:

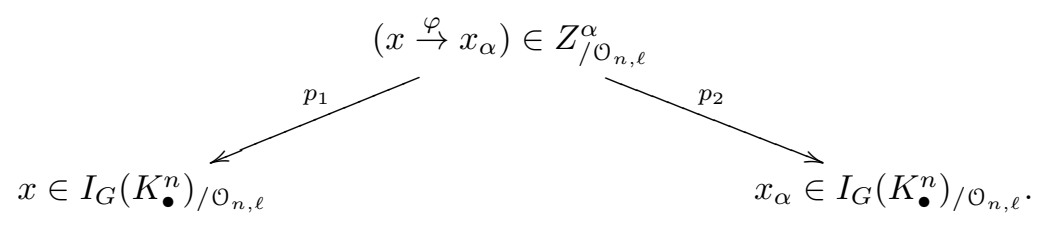

It is well known that $p_{1}$ and $p_{2}$ are Galois covering maps ( Kot92, $\left.\S 6\right]$ and [FC90, Chapter VII, §3]), so $Z^{\alpha}$ induces the algebraic correspondence $\left[K_{\bullet}^{n} \alpha K_{\bullet}^{n}\right]$ on $I_{G}\left(K_{\bullet}^{n}\right)_{/ \mathcal{O}_{n, \ell}}$.

3.7.2. Hecke operators on modular forms. Since $\ell$ is invertible in $\mathcal{O}_{n, \ell}$, the isogeny $\varphi$ is étale and induces an isomorphism $\varphi^{*}: H^{0}\left(A_{\alpha}, \Omega_{A_{\alpha / R}}\right) \cong H^{0}\left(A, \Omega_{A_{/ R}}\right)$.

Definition 3.13. For $f \in \omega_{\underline{k}}$, define the Hecke operator $f \mid\left[K_{\bullet}^{n} \alpha K_{\bullet}^{n}\right]$ by

$$
f \mid\left[K_{\bullet}^{n} \alpha K_{\bullet}^{n}\right]:=\left(p_{1}\right)_{*} \circ\left(\varphi^{*}\right)^{-1} \circ p_{2}^{*} f .
$$

If $K_{\ell}=K_{\ell}^{0}$, we write $T(\alpha)=\mid\left[K_{\bullet}^{n} \alpha K_{\bullet}^{n}\right]$. If $\ell=p$ and $K=K_{\bullet}^{n}$ for $n>0$, we write $U(\alpha)$ for $\mid\left[K_{\bullet}^{n} \alpha K_{\bullet}^{n}\right]$.

3.7.3. Hecke correspondence at $p$. We also consider the correspondence over Igusa schemes $I_{G}\left(K_{\bullet}^{n}\right)_{/ \mathcal{R}}$ for $n>0$, where $\mathcal{R}$ can be $\mathcal{K}, \mathcal{O}$ or $\mathcal{O}_{m}$. Let $X_{*}(T)_{+}=$ $\left\{\mu \in X_{*}(T) \mid\langle\chi, \mu\rangle<0\right.$ for all positive roots $\left.\chi\right\}$ and $\Delta_{p}=\left\{\mu(p) \in T\left(\mathbf{Q}_{p}\right) \mid \mu \in\right.$ $\left.X_{*}(T)_{+}\right\}$. Then $\Delta_{p}$ is a semi-group generated by $\alpha_{1}, \alpha_{2}, \ldots, \alpha_{r+s}$, where

$$
\begin{aligned}
\alpha_{i+s} & =\left(\left[\begin{array}{ll}
\mathbf{1}_{r-i} & \\
& p \cdot \mathbf{1}_{i}
\end{array}\right], \mathbf{1}_{s}\right), i=1, \ldots, r \text { and } \\
\alpha_{j} & =\left(\mathbf{1}_{r},\left[\begin{array}{ll}
p \cdot \mathbf{1}_{s-j} & \\
& \mathbf{1}_{j}
\end{array}\right]\right), j=1, \ldots, s .
\end{aligned}
$$

Let $\alpha=\alpha_{i}$ for some $i=1, \ldots, r+s$. We regard $\alpha=(a, d, p)$ as an element in $\operatorname{End}\left(M_{\Sigma}^{-1}\right) \times \operatorname{End}\left(M_{\Sigma^{c}}^{-1}\right) \times \mathcal{F}_{p}^{\times}$. Under the identification in (1.4), we further view $\alpha$ as an element in $\operatorname{End}\left(M_{p}\right) \cap \mathrm{R}_{\mathcal{F} / \mathbf{Q}} G\left(\mathbf{Q}_{p}\right)$ with $\nu(\alpha)=p$ and write

$$
\alpha=\left(a, d^{\vee}, d, a^{\vee}\right) \in \operatorname{End}\left(M_{\Sigma}^{-1}\right) \times \operatorname{End}\left(M_{\Sigma}^{0}\right) \times \operatorname{End}\left(M_{\Sigma^{c}}^{-1}\right) \times \operatorname{End}\left(M_{\Sigma^{c}}^{0}\right) .
$$

On the other hand, the action of $\alpha$ on $V_{\Sigma^{c}}$ is given by $\alpha^{\prime}$, and a simple computation shows $a^{\vee}=a^{-1} p$ and $d^{\vee}=d^{-1} p$. Under the identification $\operatorname{GL}_{r+s}\left(\mathcal{F}_{p}\right) \times \mathcal{F}_{p}^{\times}=$ $\mathrm{GL}\left(V_{\Sigma}\right) \times \mathcal{F}_{p}^{\times}=\mathrm{R}_{\mathcal{F} / \mathbf{Q}} G\left(\mathbf{Q}_{p}\right)$, we find that

$$
\alpha=\left(\left[\begin{array}{ll}
a & \\
& d^{-1} p
\end{array}\right], p\right) \in \mathrm{GL}_{r+s}\left(\mathcal{F}_{p}\right) \times \mathcal{F}_{p}^{\times} .
$$

We consider the scheme $Z_{p / \mathcal{R}}^{\alpha}$ classifying a triple $\left(x \stackrel{\varphi}{\rightarrow} x_{\alpha}\right)$ as in the previous subsection, but now $\varphi$ is a $p$-isogeny of type $\alpha$. We have $\varphi^{*} \lambda_{\alpha}=\nu(p) \lambda$ and the following commutative diagram: 


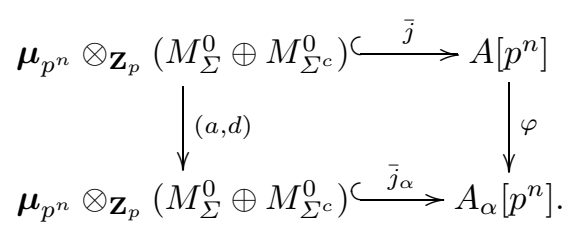

We shall call $x$ the source and $x_{\alpha}$ the target of $\left(x \stackrel{\varphi}{\rightarrow} x_{\alpha}\right)$ respectively. Consider the correspondence:

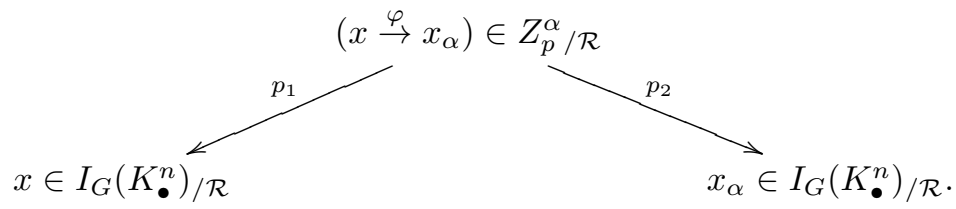

The deformation theory of ordinary abelian varieties shows that $p_{1}$ and $p_{2}$ are indeed finite and flat (cf. [Hid04b, \$8.3.1, pages 358-359] and [FC90, Chapter VII, Prop. 4.1]) and thus obtain the algebraic correspondence $\left[K_{\bullet}^{n} \alpha K_{\bullet}^{n}\right]$ on $I_{G}\left(K_{\bullet}^{n}\right) / \mathcal{R}$ induced by $Z_{p}^{\alpha}$. In addition, if $\mathcal{R}=\mathcal{O}[1 / p]$, then $Z_{p / \mathcal{R}\left[e^{2 \pi i / p^{n}}\right]}^{\alpha}=Z_{/ \mathcal{O}_{n, p}}^{\alpha}$, and the two correspondences coincide.

3.8. Hecke operators on $\boldsymbol{p}$-adic modular forms. Let $\mathcal{R}=\mathcal{O}$ and $\mathcal{O}_{m}$. We recall Hida's definition of normalized $U_{p}$-operators on the sheaf $\omega_{\underline{k}}$ over $I_{G}\left(K_{\bullet}^{n}\right)_{/ \mathcal{R}}$ with $n \geq m$.

3.8.1. We begin with some notation. Let $\alpha \in \Delta_{p}$ and let $R$ be an $\mathcal{R}$-algebra. Let $x$ : Spec $R \rightarrow I_{G}\left(K_{\bullet}^{n}\right) / \mathcal{R}$ be an $R$-point. Let $(\underline{A}, \bar{j})$ be the $R$-quintuple over $x$. We consider the fibre product

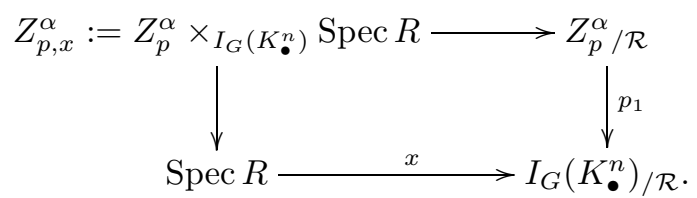

Then $Z_{p, x}^{\alpha}$ is an affine scheme which is finite and flat over Spec $R$. Let $R^{\alpha}$ be the affine coordinate ring of $Z_{p, x}^{\alpha}$ and let $\left(x \stackrel{\varphi}{\rightarrow} x_{\alpha}\right)$ be the tautological triple with the target $x_{\alpha}=\left(\underline{A}_{\alpha}, j_{\alpha}\right)$ in $Z_{p}^{\alpha}$. Note that $R^{\alpha}$ is not étale over $R$, but it can be decomposed as

$$
R^{\alpha}=\bigoplus_{u \in N_{H}\left(\mathbf{z}_{p}\right)^{\alpha} \backslash N_{H}\left(\mathbf{Z}_{p}\right)} R_{0}^{\alpha u},
$$

where $N_{H}\left(\mathbf{Z}_{p}\right)^{\alpha}=\alpha^{-1} N_{H}\left(\mathbf{Z}_{p}\right) \alpha \cap N_{H}\left(\mathbf{Z}_{p}\right)$ and $R_{0}^{\alpha u}$ is the coordinate ring of the local deformation space of $\left(\underline{A}, u^{t} j\right)$ with respect to $p$-isogeny of type $\alpha$. If $R$ is local artinian, we can determine the ring $R_{0}^{\alpha u}$ explicitly in terms of Serre-Tate coordinates (see 3.8.2).

We first consider the $\left.\right|_{k} U(\alpha)$-operator on $I_{G}\left(K_{1}^{n}\right)$ over $\mathcal{R}=\mathcal{O}_{m}$. This definition is due to Hida [Hid04b, 8.3.1]. Since $p$ is nilpotent in $\mathcal{O}_{m}$, the $p$-isogeny $\varphi: x \rightarrow x_{\alpha}$ is not étale in general. The naive definition of Hecke operators $U(\alpha)$ as algebraic correspondence in Def. 3.13 does not work over $\mathcal{O}_{m}$. To define $\left.\right|_{\underline{k}} U(\alpha)$ on the sections of the sheaf $\omega_{\underline{k}}$, we identify $\omega_{\underline{k}}$ with the $\mathbf{N}$-invariant subspace of $\mathcal{O}_{T_{n, m}} \otimes_{\mathcal{O}_{m}}$ 
$L_{\underline{k}}\left(\mathcal{O}_{m}\right)(n \geq m)$ and fix a set $\mathcal{N}_{H}^{\alpha}$ of representatives of $N_{H}\left(\mathbf{Z}_{p}\right)^{\alpha} \backslash N_{H}\left(\mathbf{Z}_{p}\right)$ in $N_{H}\left(\mathbf{Z}_{p}\right)$. For each section $f$ of $\omega_{\underline{k}}$, we define the section $\left.f\right|_{\underline{k}} U(\alpha)$ by the formulas

$$
\left.f\right|_{\underline{k}} U(\alpha)(\underline{A}, \bar{j})=\sum_{u \in \mathcal{N}_{H}^{\alpha}} \operatorname{Tr}_{R_{0}^{\alpha u} / R}\left(\rho_{\underline{k}}(u) \widetilde{\rho}_{\underline{k}}\left(\alpha^{-1}\right) f\left(\underline{A}_{\alpha u}, j_{\alpha u}\right)\right),
$$

where $\left(\underline{A}_{\alpha u}, j_{\alpha u}\right)$ is the target of the tautological triple over $R_{\alpha u}$ with the source $(\underline{A}, \bar{j})$ and $\widetilde{\rho}_{\underline{k}}\left(\alpha^{-1}\right)$ is the twisted action defined as in Remark 3.1.

Now we assume $\mathcal{R}=\mathcal{O}$. Let $R$ be a flat $\mathcal{O}$-algebra. We rephrase the definition in Def. 3.13 in terms of $L_{\underline{k}}$-valued functions on $\mathcal{E}_{V}$. For $(x, \boldsymbol{\omega}) \in \mathcal{E}_{V}(R)$, let $\boldsymbol{\omega}_{\alpha}=$ $\left(\varphi^{*}\right)^{-1}(\boldsymbol{\omega})$. Then we have

$$
f \mid U(\alpha)(x, \boldsymbol{\omega})=\operatorname{Tr}_{R^{\alpha} / R}\left(f\left(x_{\alpha}, \boldsymbol{\omega}_{\alpha}\right)\right) \in L_{\underline{k}}\left(R\left[\frac{1}{p}\right]\right) .
$$

Define the twisted operator $\left.\right|_{\underline{k}} U(\alpha)$ by

$$
\left.f(x, \boldsymbol{\omega})\right|_{\underline{k}} U(\alpha):=\underline{k}\left(\alpha^{-1}\right) \cdot f \mid U(\alpha)(x, \boldsymbol{\omega}) .
$$

We examine the $p$-integrality of $\left.\right|_{k} U(\alpha)$. Let $R_{m}=R / p^{m} R$. Then we have $R^{\alpha} / p^{m} R^{\alpha}=\left(R_{m}\right)^{\alpha}$. Recall that $\boldsymbol{\omega}(j)=\left(\boldsymbol{\omega}(j)^{+}, \boldsymbol{\omega}(j)^{-}\right):=\left(\boldsymbol{\omega}\left(j^{-}\right), \boldsymbol{\omega}\left(j^{+}\right)\right)$. From the diagram (3.15) and Remark 3.1, over $R_{m}^{\alpha}$ we have

$$
\varphi^{*}\left(\boldsymbol{\omega}(j)_{\alpha}\right)=\boldsymbol{\omega}(j) \text { and } \varphi^{*} \boldsymbol{\omega}\left(j_{\alpha}\right)=\alpha \cdot \boldsymbol{\omega}(j),
$$

which implies $\boldsymbol{\omega}\left(j_{\alpha}\right)=\alpha \cdot \boldsymbol{\omega}(j)_{\alpha}$ over $R_{m}^{\alpha}$. In other words, we have

$$
\boldsymbol{\omega}\left(j_{\alpha}\right)^{+}=d \cdot \boldsymbol{\omega}\left(j^{+}\right)_{\alpha} \text { and } \boldsymbol{\omega}\left(j_{\alpha}\right)^{+}=a \cdot \boldsymbol{\omega}\left(j^{-}\right)_{\alpha} .
$$

Let $f$ be a local section of the stalk $\left(\omega_{\underline{k}}\right)_{x}$ and choose a lifting $\boldsymbol{\omega}$ of $\boldsymbol{\omega}(j)$ over $R^{\alpha}$. From the above discussion and the fact that $R^{\alpha}$ has no $p$-torsion, we conclude that

$$
f\left(x_{\alpha}, \alpha \cdot \boldsymbol{\omega}_{\alpha}\right)=\rho_{\underline{k}}(\alpha) f\left(x_{\alpha}, \boldsymbol{\omega}_{\alpha}\right) \in L_{\underline{k}}\left(R^{\alpha}\right)
$$

and its reduction modulo $p^{m}$ is $f\left(x_{\alpha}, \boldsymbol{\omega}\left(j_{\alpha}\right)\right)$. For each $v \in L_{\underline{k}}\left(R^{\alpha}\right)$, we know $\underline{k}\left(\alpha^{-1}\right) \rho_{\underline{k}}\left(\alpha^{-1}\right) v$ is still in $L_{\underline{k}}\left(R^{\alpha}\right)$ for every $\alpha \in \Delta_{p}$. Thus $\underline{k}\left(\alpha^{-1}\right) f\left(x_{\alpha}, \omega_{\alpha}\right) \in$ $L_{\underline{k}}\left(R^{\alpha}\right)$, and

$$
\begin{aligned}
\underline{k}\left(\alpha^{-1}\right) f\left(x_{\alpha}, \omega_{\alpha}\right) & \equiv \underline{k}\left(\alpha^{-1}\right) \rho_{\underline{k}}\left(\alpha^{-1}\right) f\left(x_{\alpha}, \boldsymbol{\omega}\left(j_{\alpha}\right)\right)\left(\bmod p^{m}\right) \\
& =\widetilde{\rho}_{\underline{k}}\left(\alpha^{-1}\right) f\left(x_{\alpha}, \boldsymbol{\omega}\left(j_{\alpha}\right)\right) .
\end{aligned}
$$

Hence, the definition (3.18) is $p$-integral and is compatible with its reduction modulo $p^{m}$ as defined in (3.16).

3.8.2. Serre-Tate coordinate. Following Hid04b, §8.3.1], we explain briefly how to optimize the $p$-integrality of this twisted Hecke action by Serre-Tate theory on the deformation of ordinary abelian varieties Kat81. Let $R$ be a $p$-adic local ring with the residue field $\overline{\mathbb{F}}_{p}$. Let $x=\left[\left(A, \lambda, \iota, \bar{\eta}^{(p)}, j\right)\right]$ be an $\overline{\mathbb{F}}_{p}$-point of the unitary Igusa tower $\lim _{n} I_{U(V)}^{0}\left(K^{n}\right) \hookrightarrow{\underset{\lim }{\longleftarrow}}_{n} I_{G}^{0}\left(K^{n}\right)$. The level $p^{\infty}$-structure $j_{0}$ induces an exact sequence

$$
0 \longrightarrow M^{0} \otimes \boldsymbol{\mu}_{p^{\infty}} \longrightarrow A\left[p^{\infty}\right] \longrightarrow M^{-1} \otimes \frac{\mathbf{Q}_{p}}{\mathbf{Z}_{p}} \longrightarrow 0 .
$$

Let $q_{x}: M^{-1} \times M^{-1} \rightarrow \widehat{\mathbb{G}}_{m}$ be the Serre-Tate coordinate of $x$. In our unitary case, $q_{x}$ is determined by its restriction on $M_{\Sigma^{2}}^{-1} \times M_{\Sigma^{c}}^{-1}$. Thus the coordinate ring of the local deformation space of $x$ is given by $\widetilde{R}=R \llbracket t_{i j} \rrbracket$ with $t_{i j}=q_{x}\left(e_{i}, f_{j}\right)$. 
We compute the Serre-Tate coordinate $q_{x_{\alpha}}$ of $x_{\alpha}=\left[\left(\underline{A}_{\alpha}, j_{\alpha}\right)\right]$ for $\alpha=\alpha_{j}=(a, d)$ for $1 \leq j \leq r+s$. From the diagram (3.15), the isogeny $\varphi$ induces

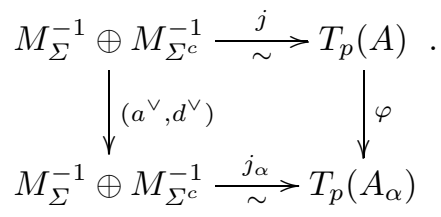

From the equation $q_{x_{\alpha}}(\varphi u, \varphi v)=q_{x}(u, v)^{p}$, we see that

$$
q_{x_{\alpha}}(u, v)=q_{x}(u a, v d)^{1 / p} \text { on } M_{\Sigma}^{-1} \times M_{\Sigma^{c}}^{-1} .
$$

Therefore, the local deformation ring of $x_{\alpha}$ is $\widetilde{R}^{\alpha}=R \llbracket t_{i j}^{\alpha} \rrbracket$ with coordinates

$$
t_{i j}^{\alpha}=q_{x}\left(e_{i} a, f_{j} d\right)^{1 / p}= \begin{cases}t_{i j}^{\frac{1}{p}} & l \leq s \text { and } i>s-l \text { or } l>s \text { and } j \geq r+s-l, \\ t_{i j} & \text { otherwise, }\end{cases}
$$

and the trace map from $\widetilde{R}^{\alpha}$ to $\widetilde{R}$ has multiplicity

$$
\chi_{r, s}(\alpha):=(\operatorname{det} a)^{-s}\left(\operatorname{det} d^{\vee}\right)^{r} .
$$

3.8.3. Hida's $U_{p}$-operators. It follows from the discussion in the previous section that the operator $\left.\right|_{\underline{k}} U(\alpha)$ is divisible by $\chi_{r, s}(\alpha)$. We recall the definition of Hida's normalized $p$-integral $U_{p}(\alpha)$-operators on the space of modular forms.

Definition 3.14. Let $\mathcal{R}=\mathcal{O}$ or $\mathcal{O}_{m}$ and $n \geq m$ be a positive integer. For $f \in$ $H^{0}\left(I_{G}\left(K_{\bullet}^{n}\right)_{/ \mathcal{R}}, \omega_{\underline{k}}\right)$, define

$$
\begin{aligned}
U_{p}(\alpha) . f & :=\left.\frac{1}{\chi_{r, s}(\alpha)} f\right|_{\underline{k}} U(\alpha) ; \\
U_{p} & =\prod_{j=1}^{r+s} U_{p}\left(\alpha_{j}\right) .
\end{aligned}
$$

We still need to consider the case $K_{\bullet}^{n}=K$, the correspondence $\left[K \alpha^{w} K_{0}^{1}\right]$ defined by the $p$-isogeny of type $\alpha^{w}$, where $w$ runs over the Weyl group of $\operatorname{GL}\left(V_{\Sigma}\right)=$ $\mathrm{GL}_{r+s}\left(\mathcal{F}_{p}\right)$ associated with $T$. In general, the correspondence $\left[K \alpha^{w} K_{0}^{1}\right]$ can be only defined over $\mathcal{K}$, and the $T(\alpha)$-operator is a sum of these $\left[K \alpha^{w} K_{0}^{1}\right]$. However, we can show easily that the normalized definition of $\left[K \alpha^{w} K_{0}^{1}\right]$ in (3.18) over $\mathcal{K}$ (sum over $\left.\alpha^{w}\right)$ still preserves the $p$-integrality. In other words, for $f \in H^{0}\left(S_{G}(K)_{/ \mathcal{O}}, \omega_{\underline{k}}\right)$, we define $U_{p}\left(\alpha^{w}\right) . f$ by the rule

$$
U_{p}\left(\alpha^{w}\right) \cdot f(\underline{A}, \boldsymbol{\omega}):=\frac{\underline{k}\left(\alpha^{-1}\right)}{\chi_{r, s}(\alpha)} f \mid\left[K \alpha^{w} K_{0}^{1}\right](\underline{A}, \boldsymbol{\omega})=\frac{\underline{k}\left(\alpha^{-1}\right)}{\chi_{r, s}(\alpha)} \operatorname{Tr}_{R^{\alpha^{w}} / R}\left(f\left(\underline{A}_{\alpha^{w}}, \boldsymbol{\omega}_{\alpha^{w}}\right)\right) .
$$

If $s=1$ and $\underline{k}=\left(a_{1}, \ldots, a_{r} ; b_{1}\right)$, there exists an integer $B(\underline{a})$ depending on $\underline{a}=$ $\left(a_{1}, \ldots a_{r}\right)$ such that $U_{p}\left(\alpha^{w}\right)$ are $p$-integral if $b_{1}>B(\underline{a}) \Sigma$. In general, it can be shown that $U_{p}\left(\alpha^{w}\right)$ are $p$-integral operators if $\underline{k}-\chi_{r, s}$ is sufficiently regular. We put

$$
T_{p}(\alpha) . f=U_{p}(\alpha) \cdot f+\sum_{w: \alpha^{w} \neq \alpha} U_{p}\left(\alpha^{w}\right) . f ; T_{p}=\prod_{j=1}^{r+s-1} T_{p}\left(\alpha_{j}\right)
$$


3.9. Hecke algebras. Let $S$ be the finite of places where $K_{v} \neq K_{v}^{0}$. For $v \notin S \cup S_{p}$, let $\mathcal{H}_{v}(G, K):=C_{c}^{\infty}\left(K_{v}^{0} \backslash G\left(\mathcal{F}_{v}\right) / K_{v}^{0}, \mathbf{Z}\right)$ be the local spherical Hecke algebra under convolution. Then $\mathcal{H}_{v}(G, K)$ acts on $f \in \mathbf{M}_{\underline{k}}\left(K_{1}^{n}, \mathbf{C}\right)$ by the formulas

$$
f * \phi(\tau, g)=\int_{G\left(\mathcal{F}_{v}\right)} f\left(\tau, g x^{-1}\right) \phi(x) d x, \phi \in \mathcal{H}_{v} .
$$

Define the global Hecke algebra $\mathcal{H}^{S}(G, K)=\bigotimes_{v \notin S \cup S_{p}}^{\prime} \mathcal{H}_{v}(G, K)$ to be the restricted tensor product of $\mathcal{H}_{v}(G, K)$ with respect to the spherical function $\mathbb{I}_{K_{v}^{0}}$. Using the identification between holomorphic modular forms and geometric modular forms in Lemma 3.7, we find that if $f \in \mathbf{M}_{\underline{k}}(U, \mathbf{C})$ and $\alpha \in G\left(\mathcal{F}_{v}\right)$ with $v \notin S \cup S_{p}$, then

$$
f \mid T(\alpha)(\tau, g)=f * \mathbb{I}_{K \alpha K}(\tau, g)=\sum_{u \in K / K \cap \alpha^{-1} K \alpha} f\left(\tau, g u \alpha^{-1}\right) .
$$

If $F \in \mathcal{A}_{\underline{k}}^{\text {hol }}(G, U, \chi)$, we define

$$
F \mid T(\alpha)(g)=\sum_{u \in K / K \cap \alpha^{-1} K \alpha} F\left(g u \alpha^{\vee}\right) \quad\left(\alpha^{\vee}=\alpha^{-1} \nu(\alpha)\right) .
$$

It is easy to verify that the inclusion $\underline{\mathrm{AM}}: \mathcal{A}_{\underline{k}}^{\text {hol }}(G, U, \chi) \hookrightarrow \mathbf{M}_{\underline{k}}^{\text {an }}(U, \mathbf{C})$ defined in Def. 3.7 is $T_{\ell}(\alpha)$-equivariant.

Similarly, we have the following formulas of the normalized Hecke operators at $p$ for holomorphic modular forms.

Proposition 3.15. Let $v \in S_{p}$. For $\alpha=\alpha_{j}, j=1, \ldots, r+s$, let

$$
\alpha_{v}:=\left(\left[\begin{array}{cc}
\mathbf{1}_{r+s-i} & 0 \\
0 & p \cdot \mathbf{1}_{i}
\end{array}\right], p\right) \in \mathrm{GL}_{r+s}\left(\mathcal{F}_{v}\right) \times \mathcal{F}_{v}^{\times}=G\left(\mathcal{F}_{v}\right) .
$$

For $f \in \mathbf{M}_{\underline{k}}^{\mathrm{an}}\left(K_{1}^{n}, \mathbf{C}\right)$, we define

$$
U_{v}(\alpha) . f(\tau, g):=\frac{\underline{k}\left(\alpha_{v}^{-1}\right)}{\chi_{r, s}\left(\alpha_{v}\right)} . \sum_{u \in N_{r+s}\left(O_{v}\right) / \alpha_{v}^{-1} N_{r+s}\left(O_{v}\right) \alpha_{v}} f\left(\tau, g u \alpha_{v}^{-1}\right) .
$$

For $f \in \mathbf{M}_{\underline{k}}^{\mathrm{an}}(K, \mathbf{C})$, we define

$$
T_{v}(\alpha) \cdot f(\tau, g):=\frac{\underline{k}\left(\alpha_{v}^{-1}\right)}{\chi_{r, s}\left(\alpha_{v}\right)} \cdot f \mid T\left(\alpha_{v}\right)(\tau, g) .
$$

Put

$$
U_{v}=\prod_{j=1}^{r+s} U_{v}\left(\alpha_{j}\right) ; T_{v}=\prod_{j=1}^{r+s} T_{p}\left(\alpha_{j}\right) .
$$

Then $U_{p}=\prod_{v \in S_{p}} U_{v}$ and $T_{p}=\prod_{v \in S_{p}} T_{v}$.

\section{HIDA THEORY FOR UNITARY GROUPS}

In this section, we develop Hida theory for modular forms on $U(r, 1)$ following Hida's approach for cusp forms (Hid02, Hid04b). Suppose that $s=1$ and $G$ is the group of unitary similitudes associated to $\left(V, \vartheta_{r, 1}\right)$. Let $\mathcal{S}=S_{G}(K)_{/ \mathcal{O}_{p}}$ be the associated unitary Shimura variety over $\mathcal{O}_{\mathfrak{p}}$. Let $\mathcal{S}^{*}=S_{G}^{*}(K) / \mathcal{O}_{\mathfrak{p}}$ and $\overline{\mathcal{S}}=\bar{S}_{G}(K)_{/ \mathcal{O}_{\mathfrak{p}}}$ be the minimal and toroidal compactifications with the structure morphism $\pi: \overline{\mathcal{S}} \rightarrow \mathcal{S}^{*}$. Let $n \geq m$ be two positive integers and write 
$\mathcal{O}_{m}=\mathcal{O}_{\mathfrak{p}} / p^{m} \mathcal{O}_{\mathfrak{p}}$. Let $\mathcal{I}=I_{G}\left(K_{1}^{n}\right) /_{\mathcal{O}}$ be the Igusa scheme of level $K_{1}^{n}$ over $\overline{\mathcal{S}}$. Let $S=\overline{\mathcal{S}}[1 / E]\left(\operatorname{resp} . \mathcal{I}_{S}=\mathcal{I}[1 / E]\right)$ and $S_{m}=S_{\mathcal{O}_{m}}\left(\right.$ resp. $\left.\mathcal{I}_{m}=\mathcal{I}_{\mathcal{O}_{m}}\right)$, where $E$ is a lifting of a power of a Hasse invariant chosen in $§ 3.3$.

4.1. A base change property. As observed by Hida, the base change property $(\mathrm{Hp} 1)$ for $\omega_{k}$ in [Hid04b, page 335] is a key ingredient in his theory. For the cuspidal part $\omega_{k}^{0}$ of $\bar{\omega}_{\underline{k}}$, this property is proved by Hida as a consequence of the description of the formal completion of $\pi_{*} \omega_{k}$ at cusps. In the case of $U(r, 1)$, the base change property does not hold for $\omega_{\underline{k}}$ unless $\mathcal{F}=\mathbf{Q}$. Nonetheless, the base change holds for a subsheaf $\omega_{k}^{b}$ which we describe as follows.

Let $\mathcal{D}=\overline{\mathcal{S}}-\mathcal{S}$. The semi-abelian scheme $\mathcal{G}$ over $\overline{\mathcal{S}}$ degenerates to the Raynaud extension $\widetilde{\mathcal{G}}$ over $\mathcal{D}$, and the restriction $\left.\underline{\omega}\right|_{\mathcal{D}}=\Omega_{\widetilde{\mathcal{G}}}$ of $\underline{\omega}$ to $\mathcal{D}$ has the decreasing filtration $\left\{\left.\mathrm{Fil}^{i} \underline{\omega}\right|_{\mathcal{D}}\right\}_{i=1,0,-1}$ defined by $\left.\mathrm{Fil}^{1} \underline{\omega}\right|_{\mathcal{D}}=\{0\},\left.\mathrm{Fil}^{0} \underline{\omega}\right|_{\mathcal{D}}=\underline{\omega}_{\mathcal{B}}$ and $\left.\mathrm{Fil}^{-1} \underline{\omega}\right|_{\mathcal{D}}=\underline{\omega}_{\mathcal{D}}$ with graded pieces $\left.\operatorname{gr}^{0} \underline{\omega}\right|_{\mathcal{D}}=\underline{\omega}_{\mathcal{B}}$ and $\left.\operatorname{gr}^{-1} \underline{\omega}\right|_{\mathcal{D}}=\Omega_{T_{X}}$. Let $\underline{k}=\left(a_{1}, a_{2}, \ldots, a_{r} ; b_{1}\right)$ be a dominant weight and let $\underline{k}^{\prime \prime}:=\left(a_{1}-a_{r}, a_{2}-\right.$ $\left.a_{r}, \ldots, a_{r-1}-a_{r}\right) \in \mathbf{Z}[\Sigma]^{r-1}$. Let $\underline{\omega}_{\mathcal{B}}^{\prime \prime}$ be the automorphic sheaf of weight $\underline{k}^{\prime \prime}$ associated to $\mathcal{B}$. Define an $\mathcal{O}_{\mathcal{D}}$-submodule $\mathscr{F}_{\mathcal{D}}$ of $\left.\omega_{\underline{k}}\right|_{\mathcal{D}}$ by

$$
\mathscr{F}_{\mathcal{D}}:=\operatorname{det}\left(\left.\underline{\omega}\right|_{\mathcal{D}}\right)^{-a_{r}} \otimes \underline{\omega}_{\mathcal{B}^{\prime \prime}} \text {. }
$$

Let $\omega_{\underline{k}}^{b}:=\left\{s \in \omega_{\underline{k}}|s|_{\mathcal{D}} \in \mathscr{F}_{\mathcal{D}}\right\}$ be a subsheaf of $\omega_{\underline{k}}$. By definition, $\omega_{\underline{k}}^{b}$ fits into the exact sequence:

$$
0 \longrightarrow \omega_{\underline{k}}^{b} \longrightarrow \omega_{\underline{k}} \longrightarrow\left(\left.\omega_{\underline{k}}\right|_{\mathcal{D}}\right) / \mathscr{F}_{\mathcal{D}} \longrightarrow 0 .
$$

Recall that the cuspidal subsheaf of $\omega_{\underline{k}}$ is given by $\omega_{\underline{k}}^{0}=\left\{s \in \omega_{\underline{k}}|s|_{\mathcal{D}}=0\right\} \subset \omega_{\underline{k}}^{b}$.

Lemma 4.1. Let $q \in\{0, b\}$. Suppose that $a_{r}+b_{1}$ is parallel, i.e. $a_{r}+b_{1}=k_{1} \Sigma$ for some $k_{1} \in \mathbf{Z}$. Then we have an isomorphism

$$
\pi_{*} \omega_{\underline{k}}^{q} \otimes \mathcal{O}_{m} \simeq \pi_{*}\left(\omega_{\underline{k}}^{q} \otimes \mathcal{O}_{m}\right) .
$$

Proof. This is proved by Hida for the cuspidal subsheaf $\omega_{\underline{k}}^{0}$. We treat the sheaf $\omega_{\underline{k}}^{b}$ following Hida's argument. Let $r: \pi_{*} \omega_{\underline{k}}^{b} \otimes \mathcal{O}_{m} \rightarrow \pi_{*}\left(\omega_{\underline{k}}^{b} \otimes \mathcal{O}_{m}\right)$ be the reduction morphism. Since $\pi: \overline{\mathcal{S}} \rightarrow \mathcal{S}^{*}$ is an isomorphism outside $\overline{\mathcal{S}}-\mathcal{S}$, it suffices to show that $r$ induces an isomorphism between the completion $\left(\pi_{*} \omega_{k}^{b}\right)_{\bar{x}}^{\wedge} \otimes \mathcal{O}_{m} \simeq \pi_{*}\left(\omega_{k}^{b} \otimes \mathcal{O}_{m}\right)_{\bar{x}}^{\wedge}$ at any geometric point $\bar{x} \in \mathcal{S}^{*}-\mathcal{S}=\partial S_{G}^{*}(K)$. Then $\bar{x}$ lies in the stratum $S_{[g]}$ for some cusp $[g]$. By the definition of $\omega_{\underline{k}}^{b}$, we have a non-canonical identification

$$
\begin{aligned}
\left(\pi_{*} \omega_{\underline{k}}^{b}\right)_{\bar{x}}^{\wedge}=\text { the } \Gamma_{[g]} \text {-fixed part of }\left\{\sum_{\beta \in \mathscr{S}_{g}^{0}} a(\beta) \mid a(0) \in A_{[g], \bar{x}} \otimes \mathscr{F}_{\mathcal{D}}\right. \text { and } \\
\\
\left.a(\beta) \in H^{0}\left(\mathcal{Z}_{[g]}^{\circ}, \mathscr{L}(\beta)\right) \otimes L_{\underline{k}}(R)\right\} .
\end{aligned}
$$

The constant term $a(0) \in \mathscr{F}_{\mathcal{D}}$ is fixed by $\Gamma_{[g]}$ and thus satisfies the relation:

$$
\rho_{\underline{k}}(\epsilon) a(0)=\epsilon^{-\left(a_{r}+b_{1}\right)} a(0)=a(0), \epsilon \in \Gamma_{[g]} .
$$


Since $a_{r}+b_{1}$ is parallel, we find that $\epsilon^{a_{r}+b_{1}}=\mathbf{N}_{\mathcal{F} / \mathbf{Q}}(\epsilon)^{k_{1}}=1$, and hence $\pi_{*}\left(\omega_{\underline{k}}^{b} \otimes R\right)_{\bar{x}}=\left\{a(0)+\sum_{\beta \in \mathscr{S}_{g}^{+}} a(\beta) \mid \rho_{\underline{k}}(\epsilon) a(\beta)=a\left(\beta \epsilon^{2}\right)\right.$ for all $\epsilon \in \Gamma_{[g]}$ and $\left.\beta \in \mathscr{S}_{g}^{+}\right\}$.

Since $\Gamma_{[g]}$ acts freely on $\mathscr{S}_{g}^{+}$and $H^{1}\left(\mathcal{Z}_{[g]}^{\circ}, \mathcal{L}(\beta)\right)=0$ for the ample line bundle $\mathcal{L}(\beta)$, the formation of $\pi_{*}\left(\omega_{\underline{k}}^{b} \otimes R\right)_{\bar{x}}$ commutes with base change. Therefore $r$ : $\pi_{*}\left(\omega_{\underline{k}}^{b} \otimes \mathcal{O}_{m}\right) \rightarrow \pi_{*} \omega_{\underline{k}}^{b} \otimes \mathcal{O}_{m}$ is an isomorphism.

Lemma 4.2. Let $q \in\{0, b\}$ and let $V_{\underline{k}}^{q}\left(K_{1}^{n}, \mathcal{O}_{m}\right):=H^{0}\left(T_{n, m}, \omega_{\underline{k}}^{q}\right)^{\mathbf{N}} \subset V_{\underline{k}}\left(K_{1}^{n}, \mathcal{O}_{m}\right)$. If $a_{r}+b_{1}$ is parallel, then we have

$$
H^{0}\left(\mathcal{I}_{S}, \omega_{\underline{k}}^{q}\right) \otimes_{\mathcal{O}_{\mathfrak{p}}} \mathcal{O}_{m}=V_{\underline{k}}^{q}\left(K_{1}^{n}, \mathcal{O}_{m}\right)
$$

Proof. Since $n \geq m>0$, the lifting $E$ of a power of Hasse invariant is nowhere vanishing on $\mathcal{I}_{\mathcal{O}_{m}}=\mathcal{I}_{S / \mathcal{O}_{m}}$. Therefore, $\mathcal{I}_{S / \mathcal{O}_{m}}=\mathcal{I}_{\mathcal{O}_{m}}$, and $H^{0}\left(\mathcal{I}_{S}, \omega_{\underline{k}}^{q} \otimes \mathcal{O}_{m}\right)=$ $H^{0}\left(\mathcal{I}_{S}, \omega_{\underline{k}}^{q} \otimes \mathcal{O}_{m}\right)=V_{\underline{k}}^{q}\left(K_{1}^{n}, \mathcal{O}_{m}\right)$. The lemma is equivalent to $H^{0}\left(\mathcal{I}_{S}, \omega_{\underline{k}}^{q}\right) \otimes \mathcal{O}_{m}=$ $H^{0}\left(\mathcal{I}_{S}, \omega_{\underline{k}}^{\bar{q}} \otimes \mathcal{O}_{m}\right)$. Consider the following diagram:

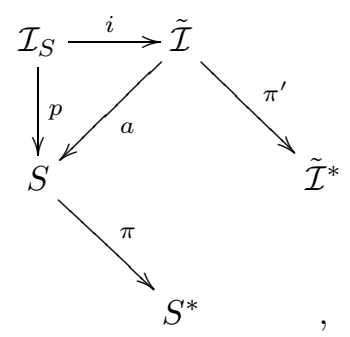

where $\tilde{\mathcal{I}}$ is the normalization of $\mathcal{I}_{S}$ above $S$ and $\tilde{\mathcal{I}}^{*}=\operatorname{Spec}(\pi \circ a)_{*} \mathcal{O}_{\mathcal{I}}$. Since $S$ is smooth over $\mathbf{Z}_{p}$, it is normal. Since $\mathcal{I}_{S}$ is étale over $S$, by [SGA71, Prop. 10.1], $i$ is an open immersion and $a$ is a finite étale covering. In addition, $a^{\prime}$ is also finite étale since $\pi_{*} \mathcal{O}_{S}=\mathcal{O}_{S^{*}}$. The morphism $\pi^{\prime}$ factors through $S \times_{S^{*}} \tilde{\mathcal{I}}^{*}$ and induces

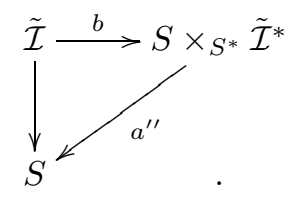

The morphism $b$ is also finite and étale since $a$ and $a^{\prime \prime}$ are finite and étale. We claim that $b$ in fact is an isomorphism. To prove the claim, it suffices to check if $d:=\operatorname{deg} a$ and $d^{\prime}:=\operatorname{deg} a^{\prime \prime}=\operatorname{deg} a^{\prime}$ are equal. Take a geometric point $y$ in $S^{*}$. Since $\pi$ has geometrically connected fibres, the map $(\pi \circ a)^{-1}(y)$ is a degree $d$ étale cover of $\pi^{-1}(y)$, and each connected component is isomorphic to $\pi^{-1}(y)$. On the other hand, $\pi^{\prime}$ is the Stein factorization of $\pi \circ a$, so $\pi^{\prime}$ also has geometrically connected fibres. It follows that $(\pi \circ a)^{-1}(y)=\left(a^{\prime} \circ \pi^{\prime}\right)^{-1}(y)$ has $d^{\prime}$ components. Hence $d=d^{\prime}$. This proves the claim.

Since $a^{\prime}$ is étale and $b$ is an isomorphism, we conclude that $\left(a^{\prime}\right)^{*} \pi_{*} \mathscr{F}=\pi_{*}^{\prime} a^{*} \mathscr{F}$ for any quasi-coherent sheaf $\mathscr{F}$ of $\bar{S}$ and also that this formation commutes with base 
change. In particular, we have $\left(a^{\prime}\right)^{*} \pi_{*} \omega_{\underline{k}}^{b}=\pi_{*}^{\prime} a^{*} \omega_{\underline{k}}^{b}$ and $\left(a^{\prime}\right)^{*} \pi_{*}\left(\omega_{\underline{k}}^{q} \otimes \mathcal{O}_{m}\right)=\pi_{*}^{\prime} a^{*}$ $\left(\omega_{\underline{k}}^{q} \otimes \mathcal{O}_{m}\right)$. Now we consider the following diagram:

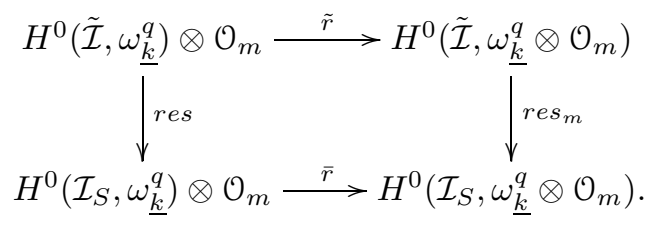

Then we have

$$
\begin{aligned}
H^{0}\left(\tilde{\mathcal{I}}, \omega_{\underline{k}}^{q}\right) \otimes \mathcal{O}_{m} & =H^{0}\left(\tilde{\mathcal{I}}^{*}, \pi_{*}^{\prime} a^{*} \omega_{\underline{k}}^{q}\right) \otimes \mathcal{O}_{m} \\
& =H^{0}\left(\tilde{\mathcal{I}}^{*},\left(a^{\prime}\right)^{*} \pi_{*} \omega_{\underline{k}}^{q} \otimes \mathcal{O}_{m}\right) \quad\left(\tilde{\mathcal{I}}^{*} \text { is affine. }\right) \\
& =H^{0}\left(\tilde{\mathcal{I}}^{*},\left(a^{\prime}\right)^{*} \pi_{*}\left(\omega_{\underline{k}}^{q} \otimes \mathcal{O}_{m}\right)\right) \quad(\text { by Lemma 4.1) } \\
& =H^{0}\left(\tilde{\mathcal{I}}^{*}, \pi_{*}^{\prime} a^{*}\left(\omega_{\underline{k}}^{q} \otimes \mathcal{O}_{m}\right)=H^{0}\left(\tilde{\mathcal{I}}, \omega_{\underline{k}}^{q} \otimes \mathcal{O}_{m}\right) .\right.
\end{aligned}
$$

This shows that $\tilde{r}$ is an isomorphism. Since $\mathcal{I}_{m}=\mathcal{I}_{S} \otimes \mathcal{O}_{m}$ is finite étale over $S_{m}, i_{m}$ is a closed immersion and hence $\mathcal{I}_{m}$ is a connected component of $\tilde{\mathcal{I}}_{m}$. This implies that $r e s_{m}$ is surjective and then $\bar{r}$ is an isomorphism.

Let $V_{n, m}^{0}$ be the cuspidal part of $V_{n, m}$. Let $V_{\infty, m}^{0}={\underline{\lim _{n}}}_{n, m} V_{n, m}^{0}$ and $V_{p}^{0}(G, K)=$ $\varliminf_{m} V_{\infty, m}^{0}$. Let $q=0$ or $\emptyset$. Applying Lemma 4.2 to the special case $\underline{k}=0$ (so $\left.\omega_{\underline{k}}^{b}=\omega_{\underline{k}}\right)$, we obtain the following exact sequence:

$$
0 \longrightarrow\left(V_{\infty, m}^{q}\right)^{\mathbf{N}} \stackrel{p}{\longrightarrow}\left(V_{\infty, m+1}^{q}\right)^{\mathbf{N}} \stackrel{p^{m}}{\longrightarrow}\left(V_{\infty, m+1}^{q}\right)^{\mathbf{N}} .
$$

We thus proved the following proposition.

Proposition 4.3. Let

$$
\mathcal{V}^{q}:=\underset{m}{\lim }\left(V_{\infty, m}^{q}\right)^{\mathbf{N}} \simeq V_{p}^{q}(G, K) \otimes \mathbf{Q}_{p} / \mathbf{Z}_{p}
$$

Then $\mathcal{V}^{q}$ is a discrete p-divisible $\mathbf{Z}_{p}$-module.

4.2. Elementary properties of the $\boldsymbol{U}_{\boldsymbol{p}}$-operator. In this subsection, we review some important properties of the $U_{p}$-operator that are established by Hida. For the sake of completeness, we reproduce some details.

Recall that $\mathbf{T}=T\left(\mathbf{Z}_{p}\right)=\left(O_{p}^{\times}\right)^{r} \times O_{p}^{\times}$. Let $\mathfrak{X}_{\mathbf{T}}:=\operatorname{Hom}_{\text {cont }}\left(\mathbf{T}, \mathbf{C}_{p}^{\times}\right)$. Throughout this section, let $\underline{k}=\left(a_{1}, \ldots, a_{r} ; b_{1}\right)$ be a dominant weight and let $\zeta=\left(\zeta_{1}, \ldots\right.$, $\left.\zeta_{r} ; \zeta_{r+1}\right) \in \mathfrak{X}_{\mathbf{T}}$ be a finite character. Suppose that $\zeta$ has level $p^{n}$, i.e. $n \in \mathbf{Z}_{+}$and $\zeta$ factors through $T\left(\mathbf{Z} / p^{n} \mathbf{Z}\right)$. We further assume that $\mathcal{O}_{\mathfrak{p}}=\mathcal{O}_{\mathfrak{p}}[\zeta]$. Regarding $\underline{k}$ as a $p$-adic character on $T\left(\mathbf{Z}_{p}\right)$ by the composition $\underline{k}: T\left(\mathbf{Z}_{p}\right) \rightarrow \overline{\mathbf{Z}}^{\times} \stackrel{\iota_{p}}{\longrightarrow} \overline{\mathbf{Z}}_{p}^{\times}$, we denote by $\underline{k}_{\zeta} \in \mathfrak{X}_{\mathbf{T}}$ the locally algebraic character $\underline{k}_{\zeta}(t)=\underline{k}(t) \zeta(t)$. We put

$$
V_{\underline{k}_{\zeta}}\left(K_{0}^{n}, \mathcal{O}_{m}\right):=\left\{f \in V_{\underline{k}}\left(K_{1}^{n}, \mathcal{O}_{m}\right) \mid[t] . f=\zeta(t) f\right\} .
$$

Proposition 4.4 (Contraction). We have

$$
U_{p} . V_{\underline{k}_{\zeta}}\left(K_{0}^{n+1}, \mathcal{O}_{m}\right) \subset V_{\underline{k}_{\zeta}}\left(K_{0}^{n}, \mathcal{O}_{m}\right) .
$$


Proof. Since $\alpha N_{H}^{t}\left(p^{n} \mathbf{Z}_{p}\right) \alpha^{-1} \subset N_{H}^{t}\left(p^{n+1} \mathbf{Z}_{p}\right)$ for every $\alpha \in \Delta_{p}$, the proposition follows from the following commutative diagram:

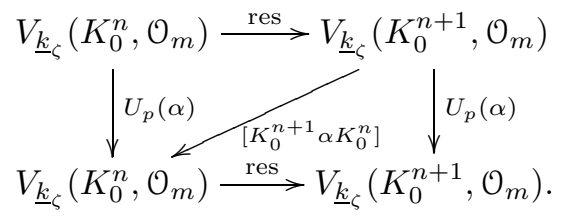

Proposition 4.5 (Commutativity with the Hasse invariant). Let $E$ be a lifting of the $t$-th power of the Hasse invariant $\bar{H}_{p}^{t}$ fixed in $\$ 3.3$. For each $f \in V_{\underline{k}}\left(K_{1}^{n}, \mathcal{O}_{m}\right)$, we have

$$
U_{p} \cdot\left(E^{l} f\right)=E^{l} \cdot U_{p} . f
$$

for a sufficiently large p-power $l$.

Proof. We may assume $n \geq m$ and identify $\left(V_{n, m} \otimes L_{\underline{k}}\left(\mathcal{O}_{m}\right)\right)^{\mathbf{N}} \simeq V_{\underline{k}}\left(K_{1}^{n}, \mathcal{O}_{m}\right)$. Let $\widehat{E}$ be the $p$-adic avatar of $E$. For a test object $(\underline{A}, \bar{j})$ in $I_{G}\left(K_{\bullet}^{n}\right)_{m}$, we have

$$
\widehat{E}(\underline{A}, \bar{j})=E(\underline{A}, \bar{j}, \boldsymbol{\omega}(j)) \equiv \bar{H}_{p-1}^{t}(\underline{A}, \bar{j}, \boldsymbol{\omega}(j))=1(\bmod p)
$$

as the $p$-th operation has trivial action on Lie $\boldsymbol{\mu}_{p^{n}}$. It follows that there exists a sufficiently large $p$-power $l$ such that for each $f \in\left(V_{n, m} \otimes L_{\underline{k}}\left(\mathcal{O}_{m}\right)\right)^{\mathbf{N}}$ with $n \geq m$, we have $f \widehat{E}^{l}=f \otimes v_{-k_{l}}$, where $k_{l}=l \cdot k_{E}$ is the weight of $E^{l}$. Since $E$ is of scalar weight, we find that

$$
U_{p} .\left(E^{l} f\right)=U_{p} . f \otimes v_{-k_{l}}=E^{l} U_{p} . f .
$$

The following lemma is based on an observation of Hida in [Hid04b, page 360].

Lemma 4.6. There is a non-negative integer a $(\underline{k})$ dependent on the weight $\underline{k}$ such that if $f \in V_{\underline{k}}\left(K_{0}^{1}, \mathcal{O}_{m}\right)$ and $\boldsymbol{\beta}_{\underline{k}}(f)=0$, then $f \equiv 0\left(\bmod p^{m-a(\underline{k})}\right)$. Furthermore, there exists $B(\underline{a}) \in \mathbf{Z}_{+}$depending on $\underline{a}:=\left(a_{1}, \ldots, a_{r}\right)$ such that if $b_{1}>B(\underline{a}) \Sigma$ and $m>a(\underline{k})$, then for every $f \in H^{0}\left(\mathcal{S}, \omega_{\underline{k}} \otimes \mathcal{O}_{m}\right)$ and a sufficiently large p-power $l$ we have

$$
T_{p}^{l} . f \equiv U_{p}^{l} \cdot f(\bmod p) .
$$

Proof. The first assertion is similar to the proof of Hid04b, Prop. 8.2]. We leave the details to the reader. The second one follows from the observation that there exists $B(\underline{a})$ such that if $b_{1}>B(\underline{a}) \Sigma$, then we have $\boldsymbol{\beta}_{\underline{k}}\left(T_{p}^{l} \cdot f\right)=\boldsymbol{\beta}_{\underline{k}}\left(U_{p}^{l} \cdot f\right)$ $(\bmod p)$ for a sufficiently large $p$-power $l$.

Proposition 4.7. If $n \geq m>0$, then the morphism

$$
\boldsymbol{\beta}_{\underline{k}}: V_{\underline{k}}\left(K_{1}^{n}, \mathcal{O}_{m}\right) \rightarrow V_{n, m}^{\mathbf{N}}
$$

defined in (3.3) is $U_{p}$-equivariant, and there exists a $\mathbf{T}$-equivariant homomorphism $\boldsymbol{s}_{\underline{k}}: V_{n, m}^{\mathbf{N}} \rightarrow V_{\underline{k}}\left(K_{1}^{n}, \mathcal{O}_{m}\right)$ such that $\boldsymbol{\beta}_{k} \circ \boldsymbol{s}_{\underline{k}}=U_{p}^{m}$ and $\boldsymbol{s}_{\underline{k}} \circ \boldsymbol{\beta}_{k}=U_{p}^{m}$. In particular, the kernel and cokernel of $\boldsymbol{\beta}_{\underline{k}}$ are annihilated by $U_{p}^{m}$. 
Proof. Let $f \in\left(V_{n, m} \otimes L_{\underline{k}}\left(\mathcal{O}_{m}\right)\right)^{\mathbf{N}}=V_{\underline{k}}\left(K_{1}^{n}, \mathcal{O}_{m}\right)$. According to the decomposition in (3.1), we write $f=\sum_{\chi \geq-\underline{k}} g_{\chi} \otimes v_{\chi}$ with $g_{\chi} \in V_{n, m}$. By definition, $\boldsymbol{\beta}_{\underline{k}}(f)=g_{-\underline{k}}$. Recall that for each $\alpha \in \Delta_{p}$ and $\mu \in X_{*}(T)_{+}$such that $\mu(p)=\alpha$, the twisted action on $v_{\chi}$ is given by

$$
\widetilde{\rho}_{\underline{k}}\left(\alpha^{-1}\right) v_{\chi}=p^{-\langle\mu, \underline{k}+\chi\rangle} v_{\chi} .
$$

We shall use the notation from 3.8.1 We define the linear map $s_{\underline{k}}(\alpha): V_{n, m}^{\mathbf{N}} \rightarrow$ $V_{n, m} \otimes L_{\underline{k}}\left(\mathcal{O}_{m}\right)$ by the rule

$$
\boldsymbol{s}_{\underline{k}}(\alpha)(f)(\underline{A}, \bar{j}):=\sum_{u \in \mathcal{N}_{H}^{\alpha}} \frac{1}{\chi_{r, 1}(\alpha)} \cdot \operatorname{Tr}_{R_{0}^{\alpha} / R}\left(f\left(\underline{A}_{\alpha u}, j_{\alpha u}\right)\right) \rho_{\underline{k}}(u) v_{-\underline{k}}
$$

for a test object $(\underline{A}, \bar{j})$ over an $\mathcal{O}_{m}$-algebra $R$. In view of (3.16), we find that $\boldsymbol{\beta}_{\underline{k}} \circ \boldsymbol{s}_{\underline{k}}(\alpha)=U_{p}(\alpha)$ on $V_{n, m}^{\mathbf{N}}$ and that

$$
\begin{aligned}
U_{p}(\alpha) \cdot f(\underline{A}, \bar{j})= & \left.\chi_{r, 1}(\alpha)^{-1} f\right|_{\underline{k}} U(\alpha)(\underline{A}, \bar{j}) \\
= & \sum_{\chi \geq-\underline{k}} \sum_{u \in \mathcal{N}_{H}^{\alpha}} \frac{1}{\chi_{r, 1}(\alpha)} \operatorname{Tr}_{R_{0}^{\alpha u} / R}\left(g_{\chi}\left(\underline{A}_{\alpha u}, j_{\alpha u}\right)\right) \otimes \rho_{\underline{k}}(u) \widetilde{\rho}_{\underline{k}}\left(\alpha^{-1}\right) v_{\chi} \\
= & s_{\underline{k}}(\alpha)\left(g_{-\underline{k}}\right)(\underline{A}, \bar{j})+\sum_{\chi>-\underline{k}, u \in \mathcal{N}_{H}^{\alpha}} p^{-\langle\mu, \underline{k}+\chi\rangle} \\
& \cdot \frac{1}{\chi_{r, 1}(\alpha)} \operatorname{Tr}_{R_{0}^{\alpha u} / R}\left(g_{\chi}\left(\underline{A}_{\alpha u}, j_{\alpha u}\right)\right) \otimes \rho_{\underline{k}}(u) v_{\chi} .
\end{aligned}
$$

This shows that $\boldsymbol{\beta}_{\underline{k}}\left(U_{p}(\alpha) . f\right)=U_{p}(\alpha) \cdot \boldsymbol{\beta}_{\underline{k}}(f)$. Let $\widetilde{\alpha}:=\prod_{i=1}^{r+s} \alpha_{i}$ and let $\boldsymbol{s}_{\underline{k}}^{z}:=$ $\boldsymbol{s}_{\underline{k}}\left(\widetilde{\alpha}^{z}\right)$ for $z \in \mathbf{Z}_{+}$. Then $U_{p}\left(\widetilde{\alpha}^{z}\right)=U_{p}^{z}$, and $\boldsymbol{\beta}_{\underline{k}} \circ \boldsymbol{s}_{\underline{k}}^{z}=U_{p}^{z}$. If $z \geq m$, then $\boldsymbol{s}_{\underline{k}}^{z}\left(V_{n, m}^{\mathbf{N}}\right) \subset V_{\underline{k}}\left(K_{1}^{n}, \mathcal{O}_{m}\right)$ and $\boldsymbol{s}_{\underline{k}}^{z} \circ \boldsymbol{\beta}_{\underline{k}}=U_{p}^{z}$ from (4.1). In addition, it is to verify that $\boldsymbol{s}_{\underline{k}}$ is $\mathbf{T}$-equivariant in the sense that $\boldsymbol{s}_{\underline{k}}([t] . f)=t^{\underline{k}}[t] \cdot \boldsymbol{s}_{\underline{k}}(f)$. Therefore, $\boldsymbol{s}_{\underline{k}}:=\boldsymbol{s}_{\underline{k}}^{m}$ is the desired homomorphism.

4.3. Construction of the ordinary projector. We shall follow the definition of the ordinary projector in Mau04, §5.3.1]. Let $R$ be an $\mathcal{O}_{\mathfrak{p}}$-algebra and let $\mathcal{T}$ be the set of ideals $I$ in $R$ such that $R / I$ is finite over $\mathcal{O}_{\mathfrak{p}}$. Then it is clear that $\mathcal{T}$ forms a fundamental system of neighborhood of 0 in $R$ and makes $R$ a topological ring. Let $R^{\dagger}$ be the completion of $R$ with respect to $\mathcal{T}$. Then $R^{\dagger}$ is a product of local rings (possibly infinitely many). To each $u \in R$ we can associate a unique idempotent $e_{u}$ in $R^{\dagger}$ such that $e_{u} u$ is invertible in $e_{u} R^{\dagger}$ while $\left(1-e_{u}\right) u$ is topologically nilpotent in $\left(1-e_{u}\right) R^{\dagger}$. Then by definition a discrete $R^{\dagger}$-module is nothing but an $R$-module in which every element is annihilated by some $I \in \mathcal{T}$. Now we consider the case $R=\mathcal{O}_{\mathfrak{p}}[X]$ and $\mathcal{T}=\left\{(p, X)^{m}\right\}_{m \in \mathbf{Z}_{+}}$.

Definition 4.8. A complete $p$-adic $X$-admissible module is an $\mathcal{O}_{\mathfrak{p}}[X]$-module $\mathcal{M}$ such that

(1) $\mathcal{M}=\lim _{m} \mathcal{M} / p^{m} \mathcal{M}$,

(2) $\mathcal{M} / p^{m} \mathcal{M}=\lim _{j} \mathcal{M}_{m, j}$, where $\mathcal{M}_{m, j}$ are discrete $\mathcal{O}_{\mathfrak{p}}\left[X^{p^{j}}\right]^{\dagger}$-modules.

Let $e_{X^{p^{j}}}$ be the idempotent in $\mathcal{O}_{\mathfrak{p}}\left[X^{p^{j}}\right]^{\dagger}$ attached to $X^{p^{j}}$. Note that $e_{X^{p^{j}}}$ is equal to the holomorphic image of $e_{X^{j+1}}$ under the natural map $\mathcal{O}_{\mathfrak{p}}\left[X^{p^{j+1}}\right]^{\dagger} \rightarrow \mathcal{O}_{\mathfrak{p}}\left[X^{p^{j}}\right]^{\dagger}$. Therefore, we obtain a well-defined idempotent $e_{X} \in$ End $M$ for each $X$-admissible 
complete $p$-adic module $\mathcal{M}$. We shall call $e_{X}$ the ordinary projector attached to $X$ (Hida's idempotent).

We sketch the existence of the ordinary projectors on the various spaces of modular forms under consideration. The $\mathcal{O}_{\mathfrak{p}}\left[T_{p}\right]$-module $H^{0}\left(\overline{\mathcal{S}}, \omega_{\underline{k}}\right)\left(\operatorname{resp} . H^{0}\left(\overline{\mathcal{S}}_{\mathcal{O}_{m}}, \omega_{\underline{k}}\right)\right)$ is finitely generated over $\mathcal{O}_{\mathfrak{p}}$ (resp. $\mathcal{O}_{m}$ ) and hence is a discrete $\mathcal{O}_{\mathfrak{p}}\left[T_{p}\right]^{\dagger}$-module. From (3.2), Prop. 4.5 and Lemma 4.6 we deduce that $V_{k}\left(K, \mathcal{O}_{m}\right)$ for $m \gg 0$ are discrete $\mathcal{O}_{\mathfrak{p}}\left[T_{p}^{p^{j}}\right]^{\dagger}=\mathcal{O}_{\mathfrak{p}}\left[U_{p}^{p^{j}}\right]^{\dagger}$-modules for $j \gg 0$. Since the cuspidal part

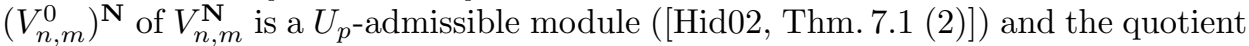
$V_{n, m}^{\mathbf{N}} /\left(V_{n, m}^{0}\right)^{\mathbf{N}}$ is a finitely generated $\mathcal{O}_{\mathfrak{p}}$-module, we find that $V_{n, m}^{\mathbf{N}}$ is $U_{p}$-admissible. This in turn shows that $V_{k}\left(K_{1}^{n}, \mathcal{O}_{m}\right)$ are $U_{p}$-admissible for all $n \geq m>0$ by Prop. 4.3 and Prop. 4.7. We thus obtain the ordinary projectors $e:=e_{U_{p}}$ and $e^{\circ}:=e_{T_{p}}$ attached to $U_{p}$ and $T_{p}$ on these modules.

Proposition 4.9. If $n \geq m>0$, then

$$
\boldsymbol{\beta}_{\underline{k}}: e \cdot V_{\underline{k}_{\zeta}}\left(K_{0}^{n}, \mathcal{O}_{m}\right) \stackrel{\sim}{\rightarrow} e \cdot V_{n, m}^{\mathbf{N}}\left[\underline{k}_{\zeta}\right] .
$$

Proof. This follows from Prop. 4.7 combined with (3.4) immediately.

Lemma 4.10 (cf. Lemma 6.8 [SU13]). If $n \geq m$, then e. $V_{\underline{k}}^{b}\left(K_{1}^{n}, \mathcal{O}_{m}\right)=$ e. $V_{\underline{k}}\left(K_{1}^{n}, \mathcal{O}_{m}\right)$.

Proof. We give a proof of the lemma for $r=2$ (i.e. $G=G U(2,1)$ ), which is the only case we need in the later application. The general case can be treated in the same way. Let $f \in e . V_{\underline{k}}\left(K_{1}^{n}, \mathcal{O}_{m}\right)$. We need to show the restriction $\left.f\left(\underline{\mathcal{M}}, j_{\mathcal{M}}\right)\right|_{\mathcal{D}}$ to $\mathcal{D}=\overline{\mathcal{S}}-\mathcal{S}$ belongs to $\mathscr{F}_{\mathcal{D}} \otimes \mathcal{O}_{\mathcal{M}} / \mathcal{J}_{+}$. Here $\left(\underline{\mathcal{M}}, j_{\mathcal{M}}\right)$ is the Mumford quintuple introduced in 2.7.4. Let $w_{2}=\left(\left[\begin{array}{ll}0 & 1 \\ 1 & 0\end{array}\right], 1\right) \in \mathbf{H}=\mathrm{GL}_{2}\left(O_{p}\right) \times O_{p}^{\times}$be the longest Weyl element. The level $p$-structure $j_{\mathcal{M}}$ gives rise to an isomorphism $\boldsymbol{\omega}\left(j_{\mathcal{M}}\right)$ : $M^{-1} \otimes \mathcal{O}_{\mathcal{M}} \simeq \underline{\omega}_{\mathcal{M}}$, and the restriction $\left.\boldsymbol{\omega}\left(j_{\mathcal{M}}\right)\right|_{\mathcal{D}}$ identifies $\mathscr{F}_{\mathcal{D}} \otimes_{\mathcal{O}_{\mathcal{D}}} \mathcal{O}_{\mathcal{M}} / \mathcal{J}_{+} \subset$ $\omega_{\underline{k}} \otimes_{\mathcal{O}_{\mathcal{D}}} \mathcal{O}_{\mathcal{M}} / \mathcal{J}_{+}$with $\mathcal{O}_{\mathcal{N}} / \mathcal{J}_{+} \cdot v_{-w_{2} \underline{k}} \subset L_{\underline{k}}\left(\mathcal{O}_{\mathcal{M}} / \mathcal{J}_{+}\right)$, the rank one module of the highest weight $-w_{2} \underline{k}=\left(-a_{2},-a_{1}\right)$. Therefore, it suffices to show that the constant term $\mathbf{a}_{[g]}^{h}(0, f)$ is of highest weight for all $h \in \mathbf{H}$ and $g \in C(K)$. We will prove this by induction on the $p$-depth of $h$ ([TU99, Def. 4.1]). Using [TU99, Lemma 4.2] and the induction hypothesis, we find that $\mathbf{a}_{[g]}^{h}(0, f)$ is a sum of $\rho_{\underline{k}}(u) \widetilde{\rho}_{\underline{k}}\left(\alpha^{-1}\right) \mathbf{a}_{\left[g_{i}\right]}^{w t}(0, f)$ for $(u, \alpha) \in N_{H}\left(\mathbf{Z}_{p}\right) \times \Delta_{p}, g_{i} \in C(K), w$ a Weyl element and $t \in \mathbf{T}$. Since the highest weight vector is an eigenfunction of $\widetilde{\rho}_{\underline{k}}\left(\alpha^{-1}\right)$ and is fixed by $N_{H}\left(\mathbf{Z}_{p}\right)$, we are reduced to showing that $\mathbf{a}_{[g]}^{w t}(0, f)$ are of highest weight for all Weyl elements $w$ in $\mathbf{H}$ and $t \in \mathbf{T}$. If $w \neq w_{2}$, then there exists $v \in S_{p}$ such that $\mathbf{a}_{[g]}^{w t}\left(0, U_{v}^{z} \cdot f\right)=p^{z} u \cdot \mathbf{a}_{[g]}(0, f)$ for some $u \in \overline{\mathbf{Z}}_{p}$, and hence $\mathbf{a}_{[g]}^{w t}(0, f)=\mathbf{a}_{[g]}^{w t}(0, e . f)=0$. If $w=w_{2}$, then we can deduce from (4.1) that

$$
\mathbf{a}_{[g]}^{w_{2} t}\left(0, U_{p}^{z} \cdot f\right)=t^{-\underline{k}} \mathbf{a}_{[g]}^{w_{2} t}\left(0, U_{p}^{z} \cdot \boldsymbol{\beta}_{\underline{k}}(f)\right) v_{-w_{2} \underline{k}} \text { for } z \geq m .
$$

We thus conclude that $\mathbf{a}_{[g]}^{w_{2} t}(0, f)=t^{-\underline{k}} \mathbf{a}_{[g]}^{w_{2} t}\left(0, \boldsymbol{\beta}_{\underline{k}}(f)\right) v_{-w_{2} \underline{k}}$. This completes the proof for $r=2$.

Proposition 4.11. Let e. $\mathcal{V}_{\underline{k}}\left(K_{1}^{n}\right):=\lim _{\longrightarrow} e \cdot V_{\underline{k}}\left(K_{1}^{n}, \mathcal{O}_{m}\right)$. Suppose that $a_{r}+b_{1}$ is parallel. Then the discrete $\mathbf{Z}_{p}$-module e. $\mathcal{V}_{\underline{k}}\left(K_{1}^{n}\right)$ is p-divisible and

$$
e . \mathcal{V}_{\underline{k}}\left(K_{1}^{n}\right)\left[p^{m}\right]=e . V_{\underline{k}}\left(K_{1}^{n}, \mathcal{O}_{m}\right)=e . H^{0}\left(\mathcal{I}_{S}, \omega_{\underline{k}}\right) \otimes \mathcal{O}_{m} .
$$


Proof. Recall that $\mathcal{I}_{S}=I_{G}\left(K_{1}^{n}\right)[1 / E]_{/ \mathcal{O}_{\mathfrak{p}}}$ with $E$ a scalar-valued modular form of weight $k_{E}$ obtained by taking a lift of a power of the Hasse invariant. By either Remark 3.10 or Lemma 3.12, $H^{0}\left(\mathcal{I}_{S / \mathbf{C}_{p}}, \omega_{k}^{b}\right)=\lim _{s} H^{0}\left(I_{G}\left(K_{1}^{n}\right) / \mathbf{C}_{p}, \omega_{k+s k_{E}}^{b}\right)$ $\left[1 / E^{s}\right]=H^{0}\left(\mathcal{I}_{S / \mathbf{C}_{p}}, \omega_{\underline{k}}\right)$, and by the flatness of $\mathbf{C}_{p}$ over $\mathcal{O}_{\mathfrak{p}}, H^{0}\left(\mathcal{I}_{S}, \omega_{\underline{k}}^{b}\right)=$ $H^{0}\left(\mathcal{I}_{S}, \omega_{\underline{k}}\right) \cap H^{0}\left(\mathcal{I}_{S / \mathbf{C}_{p}}, \omega_{\underline{k}}^{b}\right)=H^{0}\left(\mathcal{I}_{S}, \omega_{\underline{k}}\right)$. Hence, from Lemma 4.2 and Lemma 4.10 we deduce that $e . H^{0}\left(\mathcal{I}_{S}, \omega_{\underline{k}}\right) \otimes \mathcal{O}_{m}=e . V_{\underline{k}}^{b}\left(K_{1}^{n}, \mathcal{O}_{m}\right)=e . V_{\underline{k}}\left(K_{1}^{n}, \mathcal{O}_{m}\right)$ and the exact sequence

$$
0 \longrightarrow e . V_{\underline{k}}\left(K_{1}^{n}, \mathcal{O}_{m}\right) \stackrel{p}{\longrightarrow} e . V_{\underline{k}}\left(K_{1}^{n}, \mathcal{O}_{m+1}\right) \stackrel{p^{m}}{\longrightarrow} e . V_{\underline{k}}\left(K_{1}^{n}, \mathcal{O}_{m+1}\right) .
$$

It is clear that the proposition follows.

Definition 4.12. Let $q=0$ or $\emptyset$. Put $\mathbf{M}_{\underline{k}}^{q}\left(K_{1}^{n}, \mathcal{O}_{\mathfrak{p}}\right):=H^{0}\left(\mathcal{I}, \omega_{\underline{k}}^{q}\right)$. For a base ring $R$ flat over $\mathcal{O}_{\mathfrak{p}}$, define

$$
e \cdot \mathbf{M}_{\underline{k}}^{q}\left(K_{1}^{n}, R\right):=e \cdot \mathbf{M}_{\underline{k}}^{q}\left(K_{1}^{n}, \mathcal{O}_{\mathfrak{p}}\right) \otimes_{\mathcal{O}_{\mathfrak{p}}} R .
$$

Then $e \cdot \mathbf{M}_{\underline{k}}^{q}\left(K_{1}^{n}, R\right)$ has a natural $T\left(\mathbf{Z}_{p}\right)$-action. Put

$$
e \cdot \mathbf{M}_{\underline{k}_{\zeta}}^{q}\left(K_{0}^{n}, R\right):=\left\{f \in e \cdot \mathbf{M}_{\underline{k}}^{q}\left(K_{1}^{n}, R\right) \mid[t] \cdot f=\zeta(t) f\right\} .
$$

Recall that we have fixed an isomorphism $\iota: \mathbf{C} \stackrel{\sim}{\rightarrow} \mathbf{C}_{p}$. If $f \in \mathbf{M}_{\underline{k}}\left(K_{1}^{n}, \mathbf{C}\right)$, then we say $f$ is an ordinary eigenform if $f$ is an eigenvector of a $U_{p}$-operator and

$$
U_{p} . f=a_{p}(f) \cdot f \text { with } \iota\left(a_{p}(f)\right) \in \overline{\mathbf{Z}}_{p}^{\times} .
$$

Then $e \cdot \mathbf{M}_{\underline{k}}\left(K_{1}^{n}, \mathbf{C}\right)=e \cdot \mathbf{M}_{\underline{k}}\left(K_{1}^{n}, \mathcal{O}_{\mathfrak{p}}\right) \otimes_{\iota^{-1}\left(\mathcal{O}_{\mathfrak{p}}\right)} \mathbf{C}$ is the $\mathbf{C}$-vector space spanned by ordinary eigenforms of weight $\underline{k}$.

4.4. A topological control theorem. In this subsection, we prove a weak control theorem for ordinary modular forms over $\mathbf{C}$. Thanks to the control theorem of Hida for ordinary cusp forms, the remaining task is to study the control of the constant terms of ordinary modular forms.

4.4.1. Control theorems for definite unitary groups. We begin with a brief account of control theorems for the totally definite unitary group $G_{P}=G U(W)$. We retain the notation for $G U(W)$ in 3.5 and 3.6 .1 . Let $\underline{k}^{\prime}=\left(a_{1}, \ldots, a_{r-1}\right)$ be the dominant weight of $T_{P}$ associated to $\underline{k}$. Suppose that $\left\|\underline{k}^{\prime}\right\|$ is parallel. Let $R$ be a $p$-adic $\mathcal{O}_{\mathfrak{p}}$-algebra. Let $j \in\{1, \ldots, r-1\}$ and let $\alpha_{j}=\left[\begin{array}{cc}\mathbf{1}_{r-1-j} & 0 \\ 0 & p \cdot \mathbf{1}_{j}\end{array}\right] \in$ $\mathrm{GL}_{r-1}\left(\mathcal{F}_{p}\right)=G_{P}\left(\mathcal{F}_{p}\right)$. For each $\alpha=\alpha_{j}$, we fix $\mathcal{N}^{\alpha}$ a set of representatives of $N_{r-1}\left(O_{p}\right) / \alpha^{-1} N_{r-1}\left(O_{p}\right) \alpha$, and for $\widehat{\mathbf{M}}_{\underline{k}^{\prime}}(R):=\underline{\lim }_{U \subset G_{P}\left(\mathbf{A}_{\mathcal{F}, f}\right)} \widehat{\mathbf{M}}_{\underline{k}^{\prime}}(U, R)$, we define $U_{p}(\alpha) \in$ End $\widehat{\mathbf{M}}_{\underline{k}^{\prime}}(R)$ by the rule

$$
U_{p}(\alpha) \cdot f\left(g_{1}\right)=\sum_{u \in \mathcal{N}^{\alpha}} \rho_{\underline{k}^{\prime}}(u) \widetilde{\rho}_{\underline{k}^{\prime}}\left(\alpha^{-1}\right) f\left(g_{1} u \alpha^{-1}\right) \quad\left(f \in \widehat{\mathbf{M}}_{\underline{k}^{\prime}}(R), g_{1} \in G_{P}\left(\mathbf{A}_{\mathcal{F}, f}\right)\right) .
$$

The fact that $U_{p}(\alpha) . f \in \widehat{\mathbf{M}}_{\underline{k}^{\prime}}(R)$ follows from the $p$-integrality of the twisted action $\widetilde{\rho}_{\underline{k}^{\prime}}\left(\alpha^{-1}\right)=\underline{k}^{\prime}\left(\alpha^{-1}\right) \rho_{\underline{k}^{\prime}}\left(\alpha^{-1}\right)$. Let $U_{p}:=\prod_{j=1}^{r-1} U_{p}\left(\alpha_{j}\right)$ and $\mathcal{U}^{g}:=K_{P, 1}^{g, n}=$ $g K_{1}^{n} g^{-1} \cap G_{P}\left(\mathbf{A}_{\mathcal{F}, f}\right)$ for $g \in C(K)$. Then we have $U_{p} \in$ End $\widehat{\mathbf{M}}_{\underline{k}^{\prime}}\left(\mathcal{U}^{g}, R\right)$. Using the isomorphism (3.8), we obtain an operator $U_{p} \in$ End $\mathbf{M}_{\underline{k}^{\prime}}\left(\mathcal{U}^{g}, \mathbf{C}\right)$, and this $U_{p}$ agrees with the one in Prop. 3.15 for the special case $s=0$. 
Proposition 4.13. Let $e:=\lim _{n \rightarrow \infty} U_{p}^{n !}$. Then

(1) $\operatorname{dim} e \cdot \mathbf{M}_{\underline{k}^{\prime}}\left(\mathcal{U}^{g}, \mathbf{C}\right)$ is uniformly bounded independent of the weight $\underline{k}^{\prime}$.

(2) $e . \widehat{\mathbf{M}}_{\underline{k}^{\prime}}\left(K_{P, 0}^{g, 1}, \mathcal{O}_{\mathfrak{p}}\right)=e . V_{[g]}\left[\underline{k^{\prime}}\right]$.

Proof. For each $m \leq n$, consider the $U_{p}$-equivariant morphism

$$
\boldsymbol{\beta}_{\underline{\boldsymbol{k}^{\prime}}, m}: \widehat{\mathbf{M}}_{\underline{\boldsymbol{k}^{\prime}}}\left(\mathcal{U}^{g}, \mathcal{O}_{\mathfrak{p}} /\left(p^{m}\right)\right) \rightarrow A_{[g]}^{n} \otimes \mathcal{O}_{\mathfrak{p}} /\left(p^{m}\right), f \mapsto \boldsymbol{\beta}_{\underline{k}^{\prime}}(f)\left(g_{1}\right)=l_{\underline{k}^{\prime}}\left(f\left(g_{1}\right)\right) .
$$

We define $\boldsymbol{s}_{\underline{k}^{\prime}, m}: A_{[g]}^{n} \otimes \mathcal{O}_{\mathfrak{p}} /\left(p^{m}\right) \rightarrow \widehat{\mathbf{M}}_{\underline{k}^{\prime}}\left(\mathcal{U}^{g}, \mathcal{O}_{\mathfrak{p}} /\left(p^{m}\right)\right)$ by

$$
\boldsymbol{s}_{\underline{k}^{\prime}}(f)\left(g_{1}\right)=\sum_{u \in \mathcal{N}^{\alpha}} f\left(g_{1} u \alpha^{-1}\right) \otimes \rho_{\underline{k}^{\prime}}(u) v_{-\underline{k}^{\prime}} \quad\left(\alpha:=\prod_{j=1}^{r-1} \alpha_{j}\right) .
$$

From the definition of the $U_{p}$-operator, it is not difficult to see that

$$
\boldsymbol{\beta}_{\underline{k^{\prime}, m}} \circ \boldsymbol{s}_{\underline{k}^{\prime}}=U_{p} ; \boldsymbol{s}_{\underline{k}^{\prime}} \circ \boldsymbol{\beta}_{\underline{k}^{\prime}, m}=U_{p} .
$$

Therefore $\boldsymbol{\beta}_{\underline{k}^{\prime}, m}$ induces an isomorphism:

$$
e . \widehat{\mathbf{M}}_{\underline{k}^{\prime}}\left(\mathcal{U}^{g}, \mathcal{O}_{\mathfrak{p}} /\left(p^{m}\right)\right) \stackrel{\sim}{\rightarrow} e \cdot A_{[g]}^{n} \otimes \mathcal{O}_{\mathfrak{p}} /\left(p^{m}\right) .
$$

In particular, by the base change (Prop. 3.9) we have

$$
\operatorname{dim}_{\mathbf{C}} e \cdot \mathbf{M}_{\underline{k}^{\prime}}\left(\mathcal{U}^{g}, \mathbf{C}\right)=\operatorname{dim}_{\mathcal{O}_{\mathfrak{p}} /(p)} e . A_{[g]}^{n} \otimes \mathcal{O}_{\mathfrak{p}} /(p) \leq \sharp\left(G_{P}(\mathcal{F}) \backslash G_{P}\left(\mathbf{A}_{\mathcal{F}, f}\right) / \mathcal{U}^{g}\right),
$$

and part (1) follows. Moreover, we obtain the isomorphism

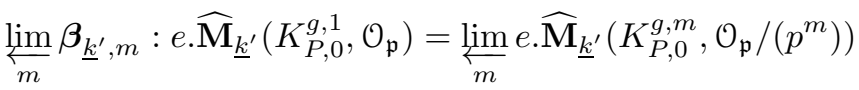

$$
\begin{aligned}
& \stackrel{\sim}{\rightarrow} \underset{m}{\lim } e \cdot A_{[g]}^{m}\left[\underline{k}^{\prime}\right] \otimes \mathcal{O}_{\mathfrak{p}} /\left(p^{m}\right)=e \cdot V_{[g]}\left[\underline{k}^{\prime}\right] .
\end{aligned}
$$

Here the first and last equalities are due to the contraction properties of $U_{p^{-}}$ operators (cf. Prop. 4.4). This proves part (2).

4.4.2. To study the constant terms of ordinary modular forms, we need to introduce some notation. Let $v \in S_{p}$ be a place above $p$ and let $F=\mathcal{F}_{v}$. For $n \in \mathbf{Z}_{+}$, let $W_{n}$ be the Weyl group with respect to the diagonal torus $T_{n}(F)$ in $\mathrm{GL}_{n}(F)$ generated by permutations of the standard basis of $F^{n}$. Denote by $\ell(w)$ the length of $w \in W_{r+1}$. Recall that we have $U(W)(F)=\mathrm{GL}_{r-1}(F)$ in $U(V)(F)=\mathrm{GL}_{r+1}(F)$ with respect to the standard basis $\left\{e_{1}, \ldots, e_{r+1}\right\}$ fixed in $\$ 1.8$ and regard $U(W)(F)$ as a subgroup of $U(V)(F)$ via $x \mapsto \operatorname{diag}(1, x, 1)$. We embed $W_{r}$ in $W_{r+1} \subset U(V)(F)$ by $x \mapsto$ $\left[\begin{array}{ll}x & \\ & 1\end{array}\right]$. For $z \in \mathbf{Z}_{+}$, let $I_{z}:=\left\{g \in \mathrm{GL}_{r+1}(F) \mid g(\bmod p) \in B_{r+1}\left(\mathcal{O}_{F} / p^{z} \mathcal{O}_{F}\right)\right\}$.

For $w \in W_{r+1}$ and $g \in G\left(\mathbf{A}_{\mathcal{F}, f}^{(p)}\right)$, put

$$
C_{w}^{g}(z):=w I_{z} \cdot P\left(\mathbf{A}_{\mathcal{F}, f}^{(p)}\right) g \subset G\left(\mathbf{A}_{\mathcal{F}, f}\right) .
$$

Recall that

$$
w_{r}=\left[\begin{array}{lll} 
& 1 & \\
\mathbf{1}_{r-1} & & \\
& & 1
\end{array}\right] \in \mathrm{GL}_{r+1}\left(\mathcal{F} \otimes_{\mathbf{Q}} \mathbf{Q}_{p}\right)=U(V)\left(\mathcal{F} \otimes_{\mathbf{Q}} \mathbf{Q}_{p}\right) .
$$


Lemma 4.14. Let $f \in e \cdot \mathbf{M}_{\underline{k}_{\zeta}}\left(K_{0}^{n}, R\right)$ and $R \subset \mathbf{C}$. For each $g \in G\left(\mathbf{A}_{\mathcal{F}, f}^{(p)}\right)$, we have

(1) $\Phi_{P, w g}(f)=0$ if $w \in W_{r}$ and $e_{1} w \neq e_{r}$.

(2) $\Phi_{[g]}^{w_{r}}(f) \in e \cdot \mathbf{M}_{\underline{k}_{\zeta}^{\prime}}\left(K_{P, 0}^{g, n}, R\right)$ is an ordinary modular form on $G_{P}$.

Proof. We may assume $R$ is a $p$-adic ring in $\mathbf{C}_{p}$. Let $W_{v}^{\prime}:=\left\{w \in W_{r} \mid e_{r} w=e_{r}\right\}$. To show part (1), it suffices to show that $\Phi_{P, w g}(f)=0$ for all $w \in W_{v}^{\prime}$. A direct computation shows that for $w \in W_{v}^{\prime}$,

$$
\Phi_{P, g w}\left(U_{p}\left(\alpha_{2}\right) \cdot f\right)=u p^{a_{r-1}-a_{r}+1} \cdot U_{p}\left(\alpha_{1}\right) \cdot \Phi_{P, g^{\prime} w}(f)
$$

for some $u \in \overline{\mathbf{Z}}_{p}^{\times}$and $g^{\prime} \in G\left(\mathbf{A}_{\mathcal{F}, f}^{(p)}\right)$. Here $U_{p}\left(\alpha_{1}\right)$ on the right-hand side is the $p$-integral operator in (4.2). This implies that $\Phi_{P, g w}(f)=0$ as $f$ is $p$-ordinary and $a_{r-1} \geq a_{r}$.

We proceed to prove part (2). Note that $\Phi_{P, w_{r} g}(f) \in \mathbf{M}_{\underline{k}_{\zeta}^{\prime}}\left(K_{P, 0}^{g, n}, R\right)$ as $w_{r}^{-1} K_{P, 0}^{g, n} w_{r} \subset K_{0}^{n}$. Let $U_{v}$ be the operator defined in Prop. 3.15] Using [TU99, Lemma 4.2], the strong approximation theorem (for $G_{P}$ ) and part (1), for each $y \in C_{w}^{g}(z)$ we can write

$$
\Phi_{P, y}\left(U_{v} \cdot f\right)=\sum_{\gamma} c_{\gamma} \cdot \rho_{\underline{k}}\left(\beta_{\gamma}\right) \Phi_{P, \gamma}(f) \quad\left(c_{\gamma} \in \mathbf{Q}(\zeta), \beta_{\gamma} \in P(\mathbf{Q})\right),
$$

where $\gamma$ runs over a finite set in $C_{w^{\prime}}^{g}\left(z^{\prime}\right), w^{\prime} \in W_{v}^{\prime}$ such that either $w^{\prime}=w$ and $z^{\prime}>z$ or $\ell\left(w^{\prime}\right)<\ell(w)$. Applying $U_{v}$ successively, for a sufficiently large $m$ we find that $\Phi_{P, y}\left(U_{v}^{m} \cdot f\right)$ is a linear combination of $\Phi_{P, w^{\prime} g^{\prime}}(f)$ for $w^{\prime} \in W_{v}^{\prime}$ and $g^{\prime} \in G\left(\mathbf{A}_{\mathcal{F}, f}^{(p)}\right)$. By part (1), we conclude that $\Phi_{P, y}(f)=0$ for all $y \in C_{w}^{g}(z)$ and $w \in W_{v}^{\prime}$. From the explicit formulas of the normalized $U_{p^{-}}$operators for $G$ and $G_{P}$ we can deduce that for $j=1, \ldots, r-2$

$$
\begin{aligned}
\Phi_{P, w_{r} g}\left(U_{p}\left(\alpha_{j+2}\right) . f\right)= & U_{p}\left(\alpha_{j}\right) \cdot \Phi_{P, w_{r} g}(f) \\
& +\sum_{v \in S_{p}}\left(\text { constant terms at } y \in C_{w^{\prime}}^{g}(1) \text { for } w^{\prime} \in W_{v}^{\prime}\right) \\
= & U_{p}\left(\alpha_{j}\right) \cdot \Phi_{P, w_{r} g}(f) .
\end{aligned}
$$

This shows that $\Phi_{[g]}^{w_{r}}(f)$ is ordinary in virtue of (3.14).

In fact, the use of [TU99, Lemma 4.2] in the proof of the above lemma yields the following consequence.

Lemma 4.15 (Lemma 4.3 TU99]). Let $g \in G\left(\mathbf{A}_{\mathcal{F}, f}^{(p)}\right)$. If $f \in e . \mathbf{M}_{\underline{k}_{\zeta}}\left(K_{0}^{n}, \mathbf{C}\right)$ and $\Phi_{P, w^{\prime} g}(f)=0$ for all $w^{\prime}$ such that $w^{\prime}=w$ or $\ell\left(w^{\prime}\right)<\ell(w)$, then $\Phi_{P, y}(f)=0$ for all $y \in C_{w}^{g}(1)$.

Theorem 4.16. Suppose that $a_{r-1}>a_{r}>r \Sigma$ (i.e. $\left.a_{r-1}-a_{r}, a_{r}-r \Sigma \in \mathbf{Z}_{+}[\Sigma]\right)$. Then we have the following exact sequence:

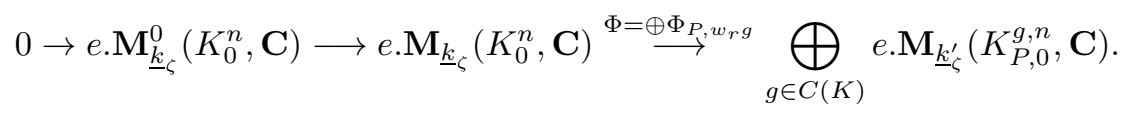

Proof. Let $f \in e \cdot \mathbf{M}_{\underline{k}_{\zeta}}\left(K_{0}^{n}, \mathbf{C}\right)$ such that $\Phi_{P, w_{r} g}(f)=0$ for all $g \in C(K)$. Let $g \in C(K) \subset G\left(\mathbf{A}_{\mathcal{F}, f}^{(p)}\right)$. To prove $f$ is a cusp form, by Bruhat decomposition, we need to show that $\Phi_{P, y}(f)=0$ for all $y \in C_{w}^{g}(1)$ and $w \in W_{r+1}$. By Lemma 4.15 it suffices to prove that $\Phi_{P, w g}(f)=0$ for all $w$ in a set of representatives of $W_{r-1} \backslash W_{r+1}$ 
in $W_{r+1}$. By Lemma 4.14 (1) and Lemma 4.15, we have $\Phi_{P, y}(f)=0$ for all $y \in C_{w}^{g}(1)$ and $w \in W_{r}$. Let $w \in W_{r+1}-W_{r}$ such that $e_{r} w \neq e_{1}$. We further assume $e_{r} w=e_{r+1}$, replacing $w$ with a suitable element in $W_{r-1} w$. Applying [TU99, Lemma 4.2], a direct computation shows that up to the multiplication by a $p$-adic unit, we have the equality

$$
\begin{aligned}
\Phi_{P, w g}\left(U_{p}\left(\alpha_{1}\right) . f\right)= & \frac{p^{a_{r-1}}}{p^{(r-1) \Sigma}} \cdot U_{p}\left(\alpha_{1}\right) \cdot \Phi_{P, w g}(f)+\sum_{\gamma} c_{\gamma}^{\prime} \cdot \rho_{\underline{k}}\left(\beta_{\gamma}^{\prime}\right) \Phi_{P, \gamma}(f) \\
& \times\left(\gamma \in C_{w^{\prime}}^{g}(1), w^{\prime} \in W_{r}, \ell\left(w^{\prime}\right)<\ell(w), c_{\gamma}^{\prime} \in \mathbf{Q}(\zeta), \beta_{\gamma}^{\prime} \in P(\mathbf{Q})\right) \\
= & \frac{p^{a_{r-1}}}{p^{(r-1) \Sigma}} \cdot U_{p}\left(\alpha_{1}\right) \cdot \Phi_{P, w g}(f) .
\end{aligned}
$$

Since $a_{r-1}>(r-1) \Sigma$, taking into account the $p$-adic counterpart of the above equation, we deduce that $\Phi_{P, w g}(f)=0$ from the $p$-ordinary property of $f$. By Lemma 4.15, we thus proved that $\Phi_{P, y}(f)=0$ for all $y \in C_{w}^{g}(1)$ and $w \in W_{r+1}$ whenever $e_{1} w \neq e_{r+1}$.

Now suppose that $e_{1} w=e_{r+1}$. Then up to a $p$-adic unit, we have

$$
\begin{aligned}
\Phi_{P, w g}\left(U_{p}\left(\alpha_{1}\right) . f\right)= & \frac{p^{a_{r}}}{p^{r \Sigma}} \cdot \Phi_{P, w g^{\prime}}(f)+\sum_{\gamma} c_{\gamma}^{\prime \prime} \cdot \rho_{\underline{k}}\left(\beta_{\gamma}^{\prime}\right) \Phi_{P, \gamma}(f) \\
& \times\left(g^{\prime} \in C(K), \gamma \in C_{w^{\prime \prime}}^{g}(1), \ell\left(w^{\prime \prime}\right)<\ell(w), c_{\gamma}^{\prime \prime} \in \mathbf{Q}(\zeta), \beta_{\gamma}^{\prime \prime} \in P(\mathbf{Q})\right) .
\end{aligned}
$$

By the induction on the length of $w$, we find that $\Phi_{P, w g}(f)=0$ as $a_{r}>r \Sigma$. This completes the proof.

Proposition 4.17. Let $B(\underline{a})$ be as in Lemma 4.6. If $b_{1}>B(\underline{a}) \Sigma$, then we have the isomorphism:

$$
e .: e^{\circ} \cdot \mathbf{M}_{\underline{k}}(K, \mathbf{C}) \stackrel{\sim}{\rightarrow} e \cdot \mathbf{M}_{\underline{k}}\left(K_{0}^{1}, \mathbf{C}\right) .
$$

Moreover, for $g \in C(K)$ we have the following commutative diagram:

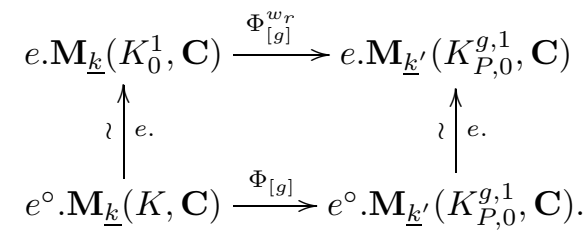

Proof. Note that Lemma 4.6 implies that we have the inclusion $e .: e^{\circ} \cdot \mathbf{M}_{\underline{k}}(K, \mathbf{C}) \rightarrow$ $e \cdot \mathbf{M}_{\underline{k}}\left(K_{0}^{1}, \mathbf{C}\right)$. Let $f \in e \cdot \mathbf{M}_{\underline{k}}\left(K_{0}^{1}, \mathbf{C}\right)$ and let $\pi=\pi_{f}$ be the automorphic representation generated by $f$. By the multiplicity one theorem of ordinary vectors [Hid04b, Thm. 5.3], we can decompose $\pi \simeq \pi^{(p)} \otimes_{v \mid p} \pi_{v}$ as a representation of $G\left(\mathbf{A}_{\mathcal{F}}^{(p)}\right) \times \prod_{v \mid p} G\left(\mathcal{F}_{v}\right)$. In addition, since $f$ is ordinary and $\underline{k}$ is dominant, $\pi_{v}$ must be an unramified and regular (i.e. the $p$-adic valuations of Satake parameters of $\pi_{v}$ are distinct) principal series for each $v \mid p\left(c f\right.$. Hid98, §5]). It follows that $f=e . f^{\circ}$ for some $f^{\circ}$ unramified at $p$. This proves the first assertion.

The second assertion follows from Lemma 4.14 (2) and the commutativity be-

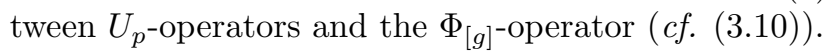

Define the space of admissible weights $\mathfrak{X}_{\mathbf{T}}^{a d m} \subset \mathfrak{X}_{\mathbf{T}}$ by

$$
\mathfrak{X}_{\mathbf{T}}^{a d m}=\left\{\underline{k}=\left(a_{1}, \ldots, a_{r} ; b_{1}\right) \mid\|\underline{k}\| \text { and } a_{r}+b_{1} \text { are parallel and } a_{r-1}>a_{r}\right\} .
$$


Proposition 4.18. The dimension of e. $\mathbf{M}_{\underline{k}}\left(K_{1}^{n}, \mathbf{C}\right)$ is uniformly bounded for all $\underline{k} \in \mathfrak{X}_{\mathbf{T}}^{a d m}$.

Proof. Let $E$ be a lifting of the $t$-th power of the Hasse invariant for a sufficiently large integer $t$ ( $c f .33 .3$. Then $E$ is a modular form of weight $k_{E}:=t(p-1)\left(I^{+}+I^{-}\right)$ and level $K$. Using Prop. 4.5, we can show that the map $f \mapsto e .(f E)$ induces an injection $e \cdot \mathbf{M}_{\underline{k}}\left(K_{1}^{n}, \mathbf{C}\right) \hookrightarrow e \cdot \mathbf{M}_{\underline{k}+k_{E}}\left(K_{1}^{n}, \mathbf{C}\right)$, so it suffices to show the uniform boundedness of $\operatorname{dim}_{\mathbf{C}} e \cdot \mathbf{M}_{\underline{k}}\left(K_{1}^{n}, \mathbf{C}\right)$ for $\underline{k} \in \mathfrak{X}_{\mathbf{T}}^{a d m}$ with $a_{r}>r \Sigma$. By the injectivity of a generalized Eichler-Shimura map ( $c f$. [Hid04b, §5.3]) and the topological control theorem [Hid95] ( $c f$. Mau04, Lemma 6.4.1]), the dimension of the space $e . \mathbf{M}_{k}^{0}\left(K_{1}^{n}, \mathbf{C}\right)$ of ordinary cusp forms is uniformly bounded for $\underline{k} \in \mathfrak{X}_{\mathbf{T}}^{a d m}$. The proposition thus follows immediately from Prop. 4.13 and Theorem 4.16.

\subsection{Control theorem for ordinary modular forms.}

Theorem 4.19 (Classicity). For each weight $\underline{k}=\left(a_{1}, \ldots, a_{r} ; b_{1}\right) \in \mathfrak{X}_{\mathbf{T}}^{\text {adm }}$, there is a positive integer $A(\underline{a}, n) \geq B(\underline{a})$ depending on $\underline{a}=\left(a_{1}, \ldots, a_{r}\right)$ and the $p$-level $n$ such that if $b_{1}>A(\underline{a}, n) \Sigma$, then the natural restriction map

$$
\text { res : e. } \mathbf{M}_{\underline{k}}\left(K_{1}^{n}, \mathcal{O}_{\mathfrak{p}}\right) \otimes \mathbf{Q}_{p} / \mathbf{Z}_{p} \stackrel{\sim}{\rightarrow} e . \mathcal{V}_{\underline{k}}\left(K_{1}^{n}\right)
$$

is an isomorphism. In particular, if $b_{1}>A(\underline{a}, 1) \Sigma$, then

$$
e^{\circ} \cdot \mathbf{M}_{\underline{k}}\left(K, \mathcal{O}_{\mathfrak{p}}\right) \otimes \mathbf{Q}_{p} / \mathbf{Z}_{p} \simeq e \cdot \mathcal{V}_{\underline{k}}\left(K_{0}^{1}\right):=\underset{\mathrm{m}}{\lim } e \cdot V_{\underline{k}}\left(K_{0}^{1}, \mathcal{O}_{m}\right) .
$$

Proof. Let $E$ be the modular form of weight $k_{E}=t(p-1)\left(I^{+}+I^{-}\right)$in Prop.4.18. We may further assume that $t$ is large enough such that there exists an everywhereunramified Hecke character $\chi$ of $\mathcal{K}^{\times}$with infinity type $t(p-1) \Sigma$. Then the function $\tilde{\chi}: X^{+} \times G\left(\mathbf{A}_{\mathcal{F}, f}\right) \rightarrow \mathbf{C}$ defined by $\tilde{\chi}\left(\tau, g_{f}\right):=\chi^{-1}\left(\operatorname{det} g_{f}\right)\left|\nu\left(g_{f}\right)\right|_{\mathbf{A}_{\mathcal{F}}}^{r t(p-1)}$ gives rise to a nowhere-vanishing modular form of weight $t(p-1)\left(-I^{+}+I^{-}\right)$. Thus $E_{1}:=E \cdot \widetilde{\chi}$ is a modular form of weight $k_{1}:=2 t(p-1) I^{-}=(0, \ldots, 0 ; 2 t(p-1) \Sigma)$ such that the multiplication by $E_{1}$ commutes with Hida's idempotent $e$.

Since $a_{r}+b_{1}$ is parallel, by Lemma 4.2 and Prop. 4.11 the restriction map res gives rise to the morphism for $m \in \mathbf{Z}_{+}$:

$$
\begin{aligned}
& \text { res }: e . H^{0}\left(\mathcal{I}, \omega_{\underline{k}}\right) \otimes \mathcal{O}_{m} \rightarrow e . H^{0}\left(\mathcal{I}_{S}, \omega_{\underline{k}}\right) \otimes \mathcal{O}_{m}=e . V_{\underline{k}}\left(K_{1}^{n}, \mathcal{O}_{m}\right) \\
& =e \cdot \mathcal{V}_{\underline{k}}\left(K_{1}^{n}\right)\left[p^{m}\right] \text {. }
\end{aligned}
$$

We need to show res is actually an isomorphism. It is injective because $\mathcal{I}_{S / \mathcal{O}_{m}}$ is open dense in $I_{G}\left(K_{\bullet}^{n}\right) / \mathcal{O}_{m}$. We shall show the surjectivity by a standard argument. Given $\bar{f} \in e \cdot H^{0}\left(\mathcal{I}_{S}, \omega_{k}^{b}\right) \otimes \mathcal{O}_{m}$ for a lift $f \in e . H^{0}\left(\mathcal{I}_{S}, \omega_{\underline{k}}\right)$, there exists a sufficiently large $p$-power $l$ such that $E_{1}^{l} f \in H^{0}\left(\mathcal{I}_{S}, \omega_{\underline{k}+l k_{1}}\right)$. Thus $e .\left(E_{1}^{l} f\right) \in$ e. $H^{0}\left(I_{G}\left(K_{\bullet}^{n}\right), \omega_{\underline{k}+l k_{1}}\right)$ and $e .\left(E_{1}^{l} f\right) \equiv E_{1}^{l} \bar{f} \equiv\left(E_{1}^{l} f\right)\left(\bmod p^{m}\right)$. Let $\mathcal{K}_{p}$ be the fractional field of $\mathcal{O}_{\mathfrak{p}}$. It follows from Prop. 4.18 that there exists $A$ depending on $\left(a_{1}, \ldots, a_{r}\right)$ such that if $b_{1}>A \Sigma$, the multiplication $E_{1}: e \cdot H^{0}\left(\mathcal{I}_{S}, \omega_{\underline{k}}\right) \otimes$ $\mathcal{K}_{p} \rightarrow e \cdot H^{0}\left(I_{G}\left(K_{\bullet}^{n}\right), \omega_{\underline{k}+k_{1}}\right) \otimes \mathcal{K}_{p}$ by $E_{1}$ is an isomorphism. Therefore, there exist $g \in e . H^{0}\left(I_{G}\left(K_{\bullet}^{n}\right), \underline{\omega}_{k}\right)$ and $u \in \mathbf{Z}_{+}$such that $p^{u} e .\left(E_{1}^{l} f\right)=E_{1}^{l} g$. Note that $p^{u}\left(E_{1}^{l} f\right) \equiv p^{u} e .\left(E_{1}^{l} f\right) \equiv E_{1}^{l} g\left(\bmod p^{m+u}\right)$, so $p^{u} f \equiv g\left(\bmod p^{m+u}\right)$. We thus conclude that $g \in p^{u} \cdot e \cdot H^{0}\left(\mathcal{I}, s, \omega_{\underline{k}}\right)$ and $f \equiv p^{-u} g\left(\bmod p^{m}\right)$. This completes the proof of the first assertion. 
To show the second assertion, note that $I_{G}\left(K_{1}^{1}\right) / \mathcal{O}_{\mathfrak{p}}$ is an étale covering over $I_{G}\left(K_{0}^{1}\right) / \mathcal{O}_{\mathfrak{p}}$ with the Galois group of prime-to- $p$ order. Hence, we can take the Galois invariant part of the isomorphism for $\mathcal{V}_{\underline{k}}\left(K_{1}^{1}\right)$ and obtain

$$
\mathcal{V}_{\underline{k}}\left(K_{0}^{1}\right) \simeq e \cdot \mathbf{M}_{\underline{k}}\left(K_{0}^{1}, \mathcal{O}_{\mathfrak{p}}\right) \otimes \mathbf{Q}_{p} / \mathbf{Z}_{p}=e^{\circ} . \mathbf{M}_{\underline{k}}\left(K, \mathcal{O}_{\mathfrak{p}}\right) \otimes \mathbf{Q}_{p} / \mathbf{Z}_{p} .
$$

Here the last equality follows from Prop. 4.17.

The above theorem leads us to the following definition of classical weights. Put

$$
\mathcal{O}_{\mathcal{F}, N_{0}}^{\times}=\left\{u \in \mathcal{O}_{\mathcal{F}}^{\times} \mid u \equiv 1\left(\bmod N_{0}\right)\right\} .
$$

We define an embedding:

$$
\begin{aligned}
j:\left(\mathcal{O}_{\mathcal{F}, N_{0}}^{\times}\right)^{2} & \longrightarrow \mathbf{T}=\left(O_{p}^{\times}\right)^{r} \times O_{p}^{\times} \\
(x, y) & \mapsto j(x, y)=\operatorname{diag}(x, \ldots, x, y, y) .
\end{aligned}
$$

Let $\mathbf{T}^{g l}$ be the $p$-adic closure of the image $j\left(\left(\mathcal{O}_{\mathcal{F}, N_{0}}^{\times}\right)^{2}\right)$ in $\mathbf{T}$ and let $\mathfrak{X}_{\mathbf{T} / \mathbf{T}^{g l}}:=$ $\operatorname{Hom}_{\text {cont }}\left(\mathbf{T} / \mathbf{T}^{g l}, \mathbf{C}_{p}^{\times}\right) \subset \mathfrak{X}_{\mathbf{T}}$. Define the space of classical weights $\mathfrak{X}_{\mathbf{T} / \mathbf{T}^{g l}}^{\text {cls }}$ by

$$
\mathfrak{X}_{\mathbf{T} / \mathbf{T}^{g l}}^{c l s}=\left\{\underline{k}_{\zeta} \in \mathfrak{X}_{\mathbf{T} / \mathbf{T}^{g l}} \mid \underline{k}=\left(a_{1}, \ldots, a_{r} ; b_{1}\right) \in \mathfrak{X}_{\mathbf{T}}^{a d m}, \zeta \text { has level } p^{n}, b_{1}>A(\underline{a}, n) \Sigma\right\} .
$$

It is clear that $\mathfrak{X}_{\mathbf{T} / \mathbf{T}^{g l}}^{c l s}$ is Zariski dense in $\mathfrak{X}_{\mathbf{T} / \mathbf{T}^{g l}}$. Let $\widetilde{\Lambda}:=\mathcal{O}_{\mathfrak{p}} \llbracket \mathbf{T} / \mathbf{T}^{g l} \rrbracket$ be a quotient ring of $\Lambda_{\mathbf{T}}=\mathcal{O}_{\mathfrak{p}} \llbracket \mathbf{T} \rrbracket$. For $\underline{k}_{\zeta} \in \mathfrak{X}_{\mathbf{T} / \mathbf{T}^{g l}}^{c l s} \subset \operatorname{Spec} \widetilde{\Lambda}\left(\mathbf{C}_{p}\right)$, let $P_{\underline{k}_{\zeta}}$ be the corresponding prime ideal of $\widetilde{\Lambda}$.

Definition 4.20. For $q=0$ or $\emptyset$, we let $V_{\text {ord }}^{q}:=\operatorname{Hom}_{\text {cont }}\left(e . \mathcal{V}^{q}, \mathbf{Q}_{p} / \mathbf{Z}_{p}\right) \otimes_{\Lambda_{\mathbf{T}}} \widetilde{\Lambda}$ and

$$
\mathcal{M}_{\text {ord }}^{q}(K, \widetilde{\Lambda}):=\operatorname{Hom}_{\widetilde{\Lambda}}\left(V_{\text {ord }}^{q}, \widetilde{\Lambda}\right) .
$$

Theorem 4.21 (The control theorem). Let $q=0$ or $\emptyset$. Then

(1) $V_{\text {ord }}^{q}$ is a free $\widetilde{\Lambda}$-module of finite rank.

(2) For every $\underline{k} \in \mathfrak{X}_{\mathbf{T} / \mathbf{T}^{g l}}^{c l s}$, we have

$$
\mathcal{M}_{\text {ord }}^{q}(K, \widetilde{\Lambda}) \otimes \widetilde{\Lambda} / P_{\underline{k}} \stackrel{\sim}{\rightarrow} e^{\circ} \cdot \mathbf{M}_{\underline{k}}^{q}\left(K, \mathcal{O}_{\mathfrak{p}}\right) .
$$

Proof. This theorem is proved by Hida for $q=0$ ([Hid04b, Thm. 8.13]). We reproduce the argument of Hida to prove the case $q=\emptyset$. It follows from Prop. 4.4, Prop. 4.9 and Theorem 4.19 that for every $\underline{k} \in \mathfrak{X}_{\mathbf{T} / \mathbf{T}^{g l}}^{\text {cls }}$, we have

$$
\begin{aligned}
e . \mathcal{V}[\underline{k}] & =\underset{m}{\lim } \underset{n}{\lim } e \cdot V_{\underline{k}}\left(K_{0}^{n}, \mathcal{O}_{m}\right)=\underset{m}{\lim } e \cdot V_{\underline{k}}\left(K_{0}^{1}, \mathcal{O}_{m}\right) \\
& =e \cdot \mathcal{V}_{\underline{k}}\left(K_{0}^{1}\right)=e^{\circ} \cdot \mathbf{M}_{\underline{k}}\left(K, \mathcal{O}_{\mathfrak{p}}\right) \otimes \mathbf{Q}_{p} / \mathbf{Z}_{p} .
\end{aligned}
$$

Taking Pontryagin dual on both sides, we find that

$$
\frac{V_{\text {ord }}}{P_{\underline{k}} V_{\text {ord }}}=\operatorname{Hom}\left(e^{\circ} \cdot \mathbf{M}_{\underline{k}}\left(K, \mathcal{O}_{\mathfrak{p}}\right) \otimes \mathbf{Q}_{p} / \mathbf{Z}_{p}, \mathbf{Q}_{p} / \mathbf{Z}_{p}\right)=\operatorname{Hom}\left(e^{\circ} \cdot \mathbf{M}_{\underline{k}}\left(K, \mathcal{O}_{\mathfrak{p}}\right), \mathcal{O}_{\mathfrak{p}}\right) .
$$

Now $V_{\text {ord }}$ is a compact $\widetilde{\Lambda}$-module such that $V_{\text {ord }} / P_{\underline{k}} V_{\text {ord }}$ is a free $\widetilde{\Lambda} / P_{\underline{k}} \widetilde{\Lambda}$ module of finite rank for all $\underline{k} \in \mathfrak{X}_{\mathbf{T} / \mathbf{T}^{g l}}^{c l s}$. Then $V_{\text {ord }}$ is a free $\widetilde{\Lambda}$-module of finite rank in virtue of the following well-known lemma. 
Lemma 4.22. Let $R$ be a complete noetherian local ring and $I_{i}$ are ideals of $R$ such that $\cap_{i} I_{i}=0$. Suppose $M$ is a compact $R$-module such that $M / I_{i} M$ are free $R / I_{i}$-modules of finite rank; then $M$ is a free $R$-module of finite rank.

This completes the proof of part (1). The specialization property in part (2) follows from (4.4) and the $\widetilde{\Lambda}$-freeness of $V_{\text {ord }}$.

We also have the control theorem for modular forms of higher levels at $p$.

Corollary 4.23. Let $\widetilde{\Lambda}_{\zeta}:=\widetilde{\Lambda} \otimes_{\mathcal{O}_{\mathfrak{p}}} \mathcal{O}_{\mathfrak{p}}[\zeta]$ and let $p^{n_{\zeta}}$ be the minimal level of $\zeta$. If $\underline{k}_{\zeta} \in \mathfrak{X}_{\mathbf{T} / \mathbf{T}^{g l}}^{\text {cls }}$, then we have

$$
\mathcal{M}_{\text {ord }}(K, \widetilde{\Lambda}) \otimes \widetilde{\Lambda}_{\zeta} / P_{\underline{k}_{\zeta}} \stackrel{\sim}{\rightarrow} e \cdot \mathbf{M}_{\underline{k}_{\zeta}}\left(K_{0}^{n_{\zeta}}, \mathcal{O}_{\mathfrak{p}}[\zeta]\right) .
$$

Proof. Let $R=\mathcal{O}_{\mathfrak{p}}[\zeta]$. Put $M=e \cdot \mathbf{M}_{\underline{k}_{\zeta}}\left(K_{0}^{n_{\zeta}}, R\right)$ and

$$
M_{m}=\underset{n}{\lim } e, V_{\underline{k}_{\zeta}}\left(K_{0}^{n}, R \otimes \mathcal{O}_{m}\right)=e \cdot V_{\underline{k}_{\zeta}}\left(K_{0}^{n_{\zeta}}, R \otimes \mathcal{O}_{m}\right) \quad \text { (by Prop. 4.4). }
$$

Let $C_{m}$ be the cokernel of the embedding $i_{m}: M \otimes \mathcal{O}_{m} \hookrightarrow M_{m}$ and let $C:=$ $\lim _{m} C_{m}$. Here the transition map $C_{m} \rightarrow C_{m+1}$ is the multiplication by $p$. We have the exact sequence:

$$
0 \longrightarrow M \otimes \frac{\mathbf{Q}_{p}}{\mathbf{Z}_{p}} \longrightarrow \underset{m}{\longrightarrow} M_{m} \longrightarrow C \longrightarrow 0
$$

Let $C^{*}$ be the Pontryagin dual of $C$. Taking the Pontryagin dual of the above exact sequence, by Prop. 4.9 we obtain

$$
0 \longrightarrow C^{*} \longrightarrow V_{\text {ord }} / P_{\underline{k}_{\zeta}} V_{\text {ord }} \longrightarrow \operatorname{Hom}_{R}(M, R) \longrightarrow 0 .
$$

We are going to show $C^{*}=0$. By Theorem 4.21, $\mathcal{M}_{\text {ord }}(K, \widetilde{\Lambda})$ is a free $\widetilde{\Lambda}$-module, so $V_{\text {ord }} / P_{\underline{k}_{\zeta}} V_{\text {ord }}$ is a free $R$-module, and $C^{*}$ is a free $\mathbf{Z}_{p}$-module. On the other hand, we claim that $C$ is killed by $\sharp\left(T\left(\mathbf{Z} / p^{n} \mathbf{Z}\right)\right)$. Indeed, let $f_{m} \in M_{m}[\zeta] \subset M_{m}$. There exists $g \in M$ such that $g \equiv f_{m}\left(\bmod p^{m}\right)$ by Theorem 4.19 (and flat base change). Let $x_{\zeta}:=\sum_{t \in T\left(\mathbf{Z} / p^{n} \mathbf{Z}\right)} \zeta^{-1}(t)[t]$ be the $\zeta$-isotypic projector. Then we have $\sharp\left(T\left(\mathbf{Z} / p^{n} \mathbf{Z}\right)\right) \cdot f_{m}=x_{\zeta} . g\left(\bmod p^{m}\right) \in M[\zeta] \otimes R_{m}$. In particular, this shows that the $C^{*}$ of $C$ is a torsion $\mathbf{Z}_{p}$-module, and hence $C^{*}=0$. This finishes the proof.

\section{6. $\widetilde{\Lambda}$-adic modular forms.}

4.6.1. $\widetilde{\Lambda}$-adic Fourier-Jacobi expansion. Let $(g, h) \in C(K) \times \mathbf{H}$ be a $p$-adic cusp such that $h^{-1} U_{P}\left(\mathbf{Z}_{p}\right) h \subset \mathbf{N}$. We consider the Fourier-Jacobi expansion of e.V :

$$
\widehat{F J}_{[g]}^{h}: \text { e.V } \longrightarrow \mathcal{R}_{[g], \infty} \otimes \mathbf{Q}_{p} / \mathbf{Z}_{p} .
$$

Let $\mathcal{R}_{[g]}(\widetilde{\Lambda}):=\mathcal{R}_{[g], \infty} \otimes_{\mathcal{O}_{\mathfrak{p}}} \widetilde{\Lambda}$. Taking the Pontryagin dual of the above equation, we obtain a morphism

$$
\operatorname{Hom}_{\widetilde{\Lambda}}\left(\mathcal{R}_{[g]}(\widetilde{\Lambda}), \widetilde{\Lambda}\right)=\operatorname{Hom}_{\mathcal{O}_{\mathfrak{p}}}\left(\mathcal{R}_{[g], \infty}, \mathcal{O}_{\mathfrak{p}}\right) \longrightarrow e . \mathcal{V}^{*}=V_{\text {ord }} .
$$

Taking the $\widetilde{\Lambda}$-dual, we obtain the $\widetilde{\Lambda}$-adic Fourier-Jacobi expansion,

$$
F J_{[g]}^{h}: \mathcal{M}_{\text {ord }}(K, \widetilde{\Lambda}) \longrightarrow \mathcal{R}_{[g]}(\widetilde{\Lambda}) .
$$


The following commutative diagram is clear from the construction of $\mathcal{M}_{\text {ord }}(K, \widetilde{\Lambda})$ :

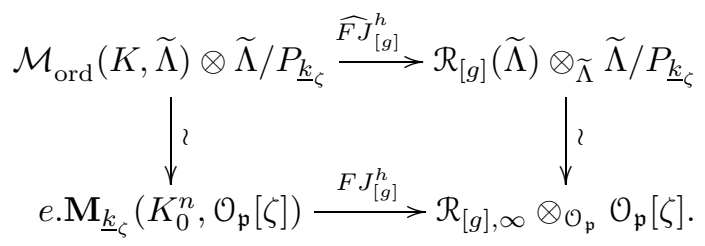

We consider the $\widetilde{\Lambda}$-adic $\widehat{\Phi}_{[g]}^{w_{r}}$-operator. Let $V_{[g] \text {,ord }}$ be the Pontryagin dual of the $p$-divisible $\Lambda_{P}$-module $e . V_{[g]} \otimes \mathbf{Q}_{p} / \mathbf{Z}_{p}$ and let

$$
\mathcal{M}_{\text {ord }}\left(K^{g}, \Lambda_{P}\right):=\operatorname{Hom}_{\Lambda_{P}}\left(V_{[g], \text { ord }}, \Lambda_{P}\right) .
$$

By Hida theory for the totally definite unitary group $G_{P}$ (Prop. 4.13 (2)), the $\Lambda_{P}$-module $\mathcal{M}_{\text {ord }}\left(K^{g}, \Lambda_{P}\right)$ is free of finite rank. We regard $\Lambda_{P}$ as a subring of $\Lambda_{\mathbf{T}}$ by the embedding $\mathbf{T}_{P} \hookrightarrow \mathbf{T}, x \mapsto \operatorname{diag}(x, 1,1)$ so that then the bottom map $\widehat{\Phi}_{[g]}^{w_{r}}: V_{\infty, m}^{\mathbf{N}} \rightarrow V_{[g]} \otimes \mathcal{O}_{m}$ in (3.10) is a $\Lambda_{P}$-module morphism. By Lemma 4.14 (2), the $\widetilde{\Lambda}$-adic Fourier-Jacobi expansion (4.5) gives rise to the $\widetilde{\Lambda}$-adic constant term map:

$$
\widehat{\Phi}_{[g]}^{w_{r}}: \mathcal{M}_{\text {ord }}(K, \widetilde{\Lambda}) \longrightarrow \mathcal{M}_{\text {ord }}\left(K_{P}^{g}, \widetilde{\Lambda}\right):=\mathcal{M}_{\text {ord }}\left(K^{g}, \Lambda_{P}\right) \otimes_{\Lambda_{P}} \widetilde{\Lambda}
$$

\subsubsection{Fundamental exact sequence.}

Definition $4.24\left(\widetilde{\Lambda}\right.$-adic modular forms). Let $\mathcal{R}_{C}^{\mathbf{T}}(\widetilde{\Lambda})=\mathcal{R}_{C}^{\mathbf{T}} \otimes_{\mathcal{O}_{\mathfrak{p}}} \widetilde{\Lambda}$. Let $\mathcal{M}_{\text {ord }}^{p \text {-adic }}$ $(K, \mathbf{T})$ be the space consisting of elements $F \in \mathcal{R}_{C}^{\mathbf{T}}(\widetilde{\Lambda})$ such that there exists a Zariski-dense subspace $\mathfrak{X}_{F} \subset \mathfrak{X}_{\mathbf{T} / \mathbf{T}^{g l}}^{\text {cls }}$ and for all $\underline{k}_{\zeta} \in \mathfrak{X}_{F}$

$$
F\left(\bmod P_{\underline{k}_{\zeta}}\right)=F J\left(f_{\underline{k}_{\zeta}}\right) \text { for some } f_{\underline{k}_{\zeta}} \in e \cdot \mathbf{M}_{\underline{k}_{\zeta}}\left(K_{0}^{n}, \mathcal{O}_{\mathfrak{p}}[\zeta]\right) \text {. }
$$

The space $\mathcal{M}_{\text {ord }}^{p \text {-adic }}(K, \mathbf{T})$ is called the space of $\widetilde{\Lambda}$-adic modular forms. The $q$ expansion principle allows us to regard $\mathcal{M}_{\text {ord }}^{p \text {-adic }}(K, \mathbf{T})$ as the space of $p$-adic measures of $\mathbf{T}$ with values in $p$-adic ordinary modular forms.

The following theorem enables us to construct $\widetilde{\Lambda}$-adic modular forms by means of the $p$-adic interpolation of Fourier-Jacobi coefficients of modular forms.

Theorem 4.25. We have a $\widetilde{\Lambda}$-module isomorphism

$$
\mathcal{M}_{\text {ord }}(K, \widetilde{\Lambda})=\mathcal{M}_{\text {ord }}^{p \text {-adic }}(K, \mathbf{T}) .
$$

Proof. We follow the argument in Hid93, Thm. 7.2]. By choosing an $\mathcal{O}_{\mathfrak{p}}$-basis of $V_{[g]} \otimes_{\mathcal{O}} H^{0}\left(\mathcal{Z}_{[g]}^{\circ} / \mathcal{O}_{\mathfrak{p}}, \mathcal{L}(\beta)\right)$ for all $g \in C(K)$ and $\beta \in \mathscr{S}_{[g]}^{0}$, we get an identification: $\mathcal{R}_{[g]}(\widetilde{\Lambda}) \stackrel{\sim}{\rightarrow} \prod_{\gamma \in J} \widetilde{\Lambda} e_{\gamma}$ for some index set $J$. Hence we can regard the Fourier-Jacobi map as a collection of $\widetilde{\Lambda}$-linear functionals on $\mathcal{M}_{\text {ord }}(K, \widetilde{\Lambda})$ via $\left\{\ell_{\gamma}\right\}_{\gamma \in J}$, the $\gamma$-th projection map $\ell_{\gamma}: \mathcal{R}_{[g]}(\widetilde{\Lambda}) \rightarrow \widetilde{\Lambda}$.

Let $f_{1}, \ldots, f_{l} \in \mathcal{M}_{\text {ord }}^{p \text {-adic }}(K, \mathbf{T})$ be linearly independent elements over $\widetilde{\Lambda}$. Let $\mathbb{K}$ be the fraction field of $\widetilde{\Lambda}$ and let $V$ be the $\mathbb{K}$-vector space spanned by $f_{1}, \ldots, f_{l}$. Then there exist $\ell_{i}=\ell_{\gamma_{i}}$ for some $\gamma_{i} \in J, i=1,2, \ldots, l$ such that $\left\{\ell_{i}\right\}_{i=1}^{l}$ is a basis of the $\mathbb{K}$-dual space of $V$. In particular, $\operatorname{det}\left(\ell_{i}\left(f_{j}\right)\right)$ is a nonzero element in $\widetilde{\Lambda}$. It follows that there exists $\underline{k}_{\zeta} \in \mathfrak{X}_{\mathbf{T} / \mathbf{T}^{g l}}^{c l s}$ such that $\operatorname{det}\left(\ell_{i}\left(f_{j}\right)\right)\left(P_{\underline{k}_{\zeta}}\right) \in \mathbb{K}^{\times}$, and 
hence the specializations $f_{1}\left(P_{\underline{k}_{\zeta}}\right), \ldots, f_{l}\left(P_{\underline{k}_{\zeta}}\right)$ are linearly independent over $\mathcal{O}_{\mathfrak{p}}[\zeta]$ in $e . \mathbf{M}_{\underline{k}_{\zeta}}\left(K, \mathcal{O}_{\mathfrak{p}}[\zeta]\right)$. By the control theorem Cor. 4.23, we thus conclude that

$\operatorname{dim}_{\mathbb{K}} \mathcal{M}_{\text {ord }}^{p \text {-adic }}(K, \mathbf{T}) \otimes_{\widetilde{\Lambda}} \mathbb{K} \leq \operatorname{rank}_{\mathcal{O}_{\mathfrak{p}}[\zeta]} e \cdot \mathbf{M}_{\underline{k}_{\zeta}}\left(K, \mathcal{O}_{\mathfrak{p}}[\zeta]\right)=\operatorname{dim}_{\mathbb{K}} \mathcal{M}_{\text {ord }}(K, \widetilde{\Lambda}) \otimes_{\widetilde{\Lambda}} \mathbb{K}$.

On the other hand, the $q$-expansion principle implies that the map $F J: \mathcal{M}_{\text {ord }}(K, \widetilde{\Lambda}) \hookrightarrow \mathcal{M}_{\text {ord }}^{p \text {-adic }}(K, \mathbf{T})$ is injective, so we have

$$
\mathcal{M}_{\text {ord }}^{p \text {-adic }}(K, \mathbf{T}) \otimes_{\widetilde{\Lambda}} \mathbb{K}=\mathcal{M}_{\text {ord }}(K, \widetilde{\Lambda}) \otimes_{\widetilde{\Lambda}} \mathbb{K} .
$$

Let $F_{1}, \ldots, F_{t}$ be a $\widetilde{\Lambda}$-basis of $\mathcal{M}_{\text {ord }}(K, \widetilde{\Lambda})$ and $\left\{\ell_{i}=\ell_{\beta_{i}}\right\}_{i=1}^{t}$ be a $\mathbb{K}$-basis of the dual space of $\mathcal{M}_{\text {ord }}(K, \widetilde{\Lambda}) \otimes \mathbb{K}$. For each $F \in \mathcal{M}_{\text {ord }}^{p \text {-adic }}(K, \mathbf{T})$, we have $\operatorname{det}\left(\ell_{i}\left(F_{j}\right)\right)$. $F \in \mathcal{M}_{\text {ord }}(K, \widetilde{\Lambda})$. It follows that $\mathcal{M}_{\text {ord }}^{p \text {-adic }}(K, \mathbf{T})$ is also a free $\widetilde{\Lambda}$-module of finite type and that

$$
\mathcal{M}^{\prime \prime}:=\mathcal{M}_{\text {ord }}^{p \text {-adic }}(K, \mathbf{T}) / \mathcal{M}_{\text {ord }}(K, \widetilde{\Lambda})
$$

is $\widetilde{\Lambda}$-torsion. Let $\mathfrak{m}$ be a maximal ideal of $\widetilde{\Lambda}$ and let $\mathbf{k}=\widetilde{\Lambda} / \mathfrak{m}$. By the $\widetilde{\Lambda}$-freeness of $\mathcal{M}_{\text {ord }}^{p \text {-adic }}(K, \mathbf{T})$, we deduce the exact sequence

$$
0 \longrightarrow \operatorname{Tor}\left(\mathcal{M}^{\prime \prime}, \mathbf{k}\right) \longrightarrow e \cdot \mathbf{M}_{\underline{k}}(K, \mathbf{k}) \stackrel{F J \otimes \mathbf{k}}{\longrightarrow} \mathcal{M}_{\text {ord }}^{p \text {-adic }}(K, \mathbf{T}) \otimes \mathbf{k} \longrightarrow \mathcal{M}^{\prime \prime} \otimes \mathbf{k} \longrightarrow 0 .
$$

The Fourier-Jacobi map $F J \otimes \mathbf{k}$ is injective by the $q$-expansion principle, so we conclude that $\operatorname{Tor}\left(\mathcal{M}^{\prime \prime}, \widetilde{\Lambda} / \mathfrak{m}\right)=0$ for every maximal ideal $\mathfrak{m}$ of $\widetilde{\Lambda}$. This implies that $\mathcal{M}^{\prime \prime}$ is $\widetilde{\Lambda}$-flat. On the other hand $\mathcal{M}^{\prime \prime}$ is $\widetilde{\Lambda}$-torsion, so we conclude that $\mathcal{M}^{\prime \prime}=0$.

The following theorem is a key ingredient in the proof of Theorem 7.18 .

Theorem 4.26 (Fundamental exact sequence).

$$
0 \longrightarrow \mathcal{M}_{\text {ord }}^{0}(K, \widetilde{\Lambda}) \longrightarrow \mathcal{M}_{\text {ord }}(K, \widetilde{\Lambda}) \stackrel{\widehat{\Phi}^{w_{r}}=\oplus \widehat{\Phi}_{[g]}^{w_{r}}}{\longrightarrow} \bigoplus_{g \in C(K)} \mathcal{M}_{\text {ord }}\left(K_{P}^{g}, \widetilde{\Lambda}\right) \longrightarrow 0
$$

Proof. In virtue of Theorem 4.16 and the control theorem, it remains to show that the Siegel operator $\Phi:=\widehat{\Phi}^{w_{r}}$ is surjective. Note that $\widetilde{\Lambda}$ is a semi-local ring with maximal ideals parametrized by the characters of $T\left(\mathbb{F}_{p}\right)$. For a character $\xi$ of $T\left(\mathbb{F}_{p}\right)$, let $\mathfrak{m}_{\xi}$ be the corresponding maximal ideal with the residue field $\mathbf{k}$. By Nakayama's lemma, it suffices to show that $\Phi\left(\bmod \mathfrak{m}_{\xi}\right)=\Phi \otimes \mathbf{k}$ is surjective for all $\xi$. For each $\xi$, we can choose an algebraic character $\underline{k} \in \mathfrak{X}_{\mathbf{T} / \mathbf{T}^{g l}}^{c l s}$ such that $\underline{k} \equiv \xi(\bmod p)$. From Theorem 4.19 and Theorem 4.21, we deduce that

$$
\begin{aligned}
\mathcal{M}_{\text {ord }}(K, \widetilde{\Lambda}) \otimes \widetilde{\Lambda} / \mathfrak{m}_{\xi} & =e^{\circ} \cdot H^{0}\left(\overline{\mathcal{S}}, \omega_{\underline{k}}\right) \otimes \mathbf{k}=e \cdot H^{0}\left(\mathcal{S}[1 / E], \omega_{\underline{k}} \otimes \mathbf{k}\right) \quad\left(e \equiv e^{\circ}(\bmod p)\right) \\
& =e \cdot H^{0}\left(S^{*}, \pi_{*}\left(\omega_{\underline{k}} \otimes \mathbf{k}\right)\right)
\end{aligned}
$$

and

$$
\bigoplus_{g \in C(K)} \mathcal{M}_{\text {ord }}\left(K_{P}^{g}, \widetilde{\Lambda}\right) \otimes \widetilde{\Lambda} / \mathfrak{m}_{\xi}=e \cdot H^{0}\left(\partial S^{*}, \pi_{*}\left(\omega_{\underline{k}}\right)\right) \otimes \mathbf{k}=e \cdot H^{0}\left(\partial S^{*}, \pi_{*}\left(\omega_{\underline{k}} \otimes \mathbf{k}\right)\right) .
$$

From the fact that the $\Phi$-operator is simply the restriction map $\left.f \rightarrow f\right|_{\partial S^{*}}$ and $S^{*}$ is an affine scheme, it follows that the map

$$
e . H^{0}\left(S^{*}, \pi_{*}\left(\omega_{\underline{k}} \otimes \mathbf{k}\right)\right) \stackrel{\Phi \otimes \mathbf{k}}{\longrightarrow} e \cdot H^{0}\left(\partial S^{*}, \pi_{*}\left(\omega_{\underline{k}} \otimes \mathbf{k}\right)\right)
$$

is surjective. 


\section{Ordinary $p$-Adic Eisenstein series on $U(2,1)$}

In this section, we construct a special $\Lambda$-adic Eisenstein series $\mathcal{E}^{\text {ord }}(\Psi \mid \mathbf{1}, \mathfrak{n})$ on $U(2,1)$. Following the method in [Hsi11], we first construct a $p$-adic Eisenstein series $\mathcal{E}_{2,2}$ for $G U(2,2)$ via the $p$-adic interpolation of Fourier coefficients (Theorem [5.8), and the desired $\Lambda$-adic Eisenstein series is obtained by applying the pull-back formula and the ordinary projector to $\mathcal{E}_{2,2}$. The main result is Cor. 5.15

\subsection{Notation and conventions.}

5.1.1. Throughout we let $\underline{W}=\left(W, \vartheta, L, L^{-1} \oplus L^{0}\right), \underline{V}=\left(V, \vartheta_{2,1}, M, M^{-1} \oplus M^{0}\right)$ and $\underline{\mathbf{W}}=\left(\mathbf{W}, \boldsymbol{\eta}_{2}, \mathbf{L}, \mathbf{L}^{-1} \oplus \mathbf{L}^{0}\right)$ be the quadruples introduced in $\$ 2.6$ for $r=2$ and $s=1$. Here $L=\mathcal{O}_{\mathcal{K}} w^{1}$ is the standard $\mathcal{O}_{\mathcal{K}}$-lattice in $W$ and $\vartheta:=\vartheta\left(w^{1}, w^{1}\right)$ is an element in $\mathcal{K}$ such that

(h1) $\bar{\vartheta}=-\vartheta$,

(h2) $\sqrt{-1} \sigma(\vartheta)>0$ and $\iota_{p}(\sigma(\vartheta))$ is a $p$-adic unit for each $\sigma \in \Sigma$.

Fix a prime-to- $p$ integral ideal $\mathfrak{c}$ of $\mathcal{O}_{\mathcal{K}}$. We introduce an auxiliary prime-to-p proper ideal $\mathfrak{n}$ of $\mathcal{O}_{\mathcal{F}}$ of the form

$$
\mathfrak{n}=\left(\mathfrak{s c} \overline{\mathfrak{c}} D_{\mathcal{K} / \mathcal{F}}\right)^{m_{0}}, m_{0}>4[\mathcal{F}: \mathbf{Q}],
$$

where $\mathfrak{s}$ is a product of primes split in $\mathcal{K}$. Let $\mathfrak{b}_{\vartheta}:=\mathcal{O}_{\mathcal{F}} \cap \vartheta \mathcal{D}_{\mathcal{K} / \mathcal{F}}^{-1}$. In addition to (h1) and (h2), we further assume $\vartheta$ satisfies

$$
\mathfrak{b}_{\vartheta, v}=\mathcal{O}_{\mathcal{F}_{v}} \text { for each } v \mid \mathfrak{n} \text {. }
$$

The lattice $M=\mathcal{O}_{\mathcal{K}} y^{1} \oplus \mathcal{O}_{\mathcal{K}} w^{1} \oplus \mathcal{D}_{\mathcal{K} / \mathcal{F}}^{-1} \vartheta x^{1}$ is an $\mathcal{O}_{\mathcal{F}}$-maximal $\mathcal{O}_{\mathcal{K}}$-lattice with respect to the Hermitian form $\vartheta^{-1} \vartheta_{2,1}$ (cf. Shi97, Lemma 4.9 (2)]). In the remainder of this article, we let

$$
\mathbf{A}=\mathbf{A}_{\mathcal{F}} \text { and } \mathbf{A}_{f}=\mathbf{A}_{\mathcal{F}, f} .
$$

5.1.2. Measures. Let $F$ be a local field. If $F$ is non-archimedean, the Haar measure $d x$ (resp. $\left.d^{\times} x\right)$ on $F$ (resp. $\left.F^{\times}\right)$is normalized so that $\operatorname{vol}\left(\mathcal{O}_{F}, d x\right)=1$ (resp. $\left.\operatorname{vol}\left(\mathcal{O}_{F}^{\times}, d^{\times} x\right)=1\right)$. If $F=\mathbf{R}$, then $d x$ is the Lebesgue measure on $\mathbf{R}$. If $F=\mathbf{C}$, then $d x$ is twice the Lebesgue measure. The absolute value $|\cdot|_{F}$ on $F$ is normalized so that $d(a x)=|a|_{F} d x$ for $a \in F$. We often drop the subscript $F$ from the notation when it is clear from the context.

For a place $v$ of $\mathcal{F}, \mathcal{H}_{2}\left(\mathcal{F}_{v}\right)$ is the set of the $2 \times 2$ Hermitian matrices in $M_{2}\left(\mathcal{K}_{v}\right)$ defined in $\$ 1.2$. The Haar measure $d X_{v}$ on $\mathcal{H}_{2}\left(\mathcal{F}_{v}\right)$ is normalized so that $\operatorname{vol}\left(\mathcal{H}_{2}\left(O_{v}\right), d X_{v}\right)=1$, and the Haar measure $d X$ on $\mathcal{H}_{2}(\mathbf{A})=\mathcal{H}_{2}\left(\mathbf{A}_{\mathcal{F}}\right)$ is normalized so that the quotient measure $\operatorname{vol}\left(\mathcal{H}_{2}(\mathbf{A}) / \mathcal{H}_{2}(\mathcal{F}), d X\right)=1$. It is well known that

$$
d X=c_{2}(\mathcal{K}) \cdot \otimes_{v} d X_{v}, \text { where } c_{2}(\mathcal{K})=2^{[\mathcal{F}: \mathbf{Q}]}\left|D_{\mathcal{F}}\right|_{\mathbf{R}}^{-1}\left|D_{\mathcal{K}}\right|_{\mathbf{R}}^{-1 / 2}
$$

(cf. [Shi97, page $153(18.9 .3)])$.

5.1.3. Let $\psi_{\mathbf{Q}}=\prod \psi_{p}$ be the standard additive character of $\mathbf{A}_{\mathbf{Q}} / \mathbf{Q}$ such that $\boldsymbol{\psi}_{\infty}\left(x_{\infty}\right)=\exp \left(2 \pi i x_{\infty}\right) \quad\left(x_{\infty} \in \mathbf{R}\right)$. Define the additive character $\boldsymbol{\psi}=\prod \boldsymbol{\psi}_{v}$ of $\mathbf{A} / \mathcal{F}$ by

$$
\psi:=\psi_{\mathbf{Q}} \circ \operatorname{Tr}_{\mathcal{F} / \mathbf{Q}}
$$


For $\beta \in \mathcal{H}_{2}(\mathcal{F})$, define the additive character $\boldsymbol{\psi}_{\beta}: \mathcal{H}_{2}(\mathbf{A}) \rightarrow \mathbf{C}$ by $\boldsymbol{\psi}_{\beta}(X):=$ $\boldsymbol{\psi}(\operatorname{Tr}(\beta X))$. If $\mathcal{L}$ is a lattice in $\mathcal{H}_{2}\left(\mathcal{F}_{v}\right)$, define the dual lattice $\mathcal{L}^{\vee}$ by

$$
\mathcal{L}^{\vee}=\left\{x \in \mathcal{H}_{2}\left(\mathcal{F}_{v}\right) \mid \boldsymbol{\psi}_{v}(\operatorname{Tr}(x y))=1 \text { for all } y \in \mathcal{L}\right\} .
$$

5.1.4. In this section, we also write $\mathfrak{r}$ for $\mathcal{O}_{\mathcal{K}}$ and $\mathfrak{d}$ for $\mathcal{D}_{\mathcal{K} / \mathcal{F}}$ especially when they appear in the entry of matrices. If $R$ is a ring and $\mathfrak{a}$ is a subset in $R$, for $x \in M_{n \times m}(R)$, we write

$$
x \prec \mathfrak{a} \Longleftrightarrow x_{i j} \in \mathfrak{a}, \forall i, j .
$$

If $x=\left[\begin{array}{ll}a & b \\ c & d\end{array}\right] \in M_{4}(R)$ with $a, b, c, d \in M_{2}(R)$, we write $a_{x}=a, b_{x}=b, c_{x}=c$ and $d_{x}=d$.

We use the notation $\mathbb{I}_{T}$ to denote the characteristic function of a set $T$.

\subsection{Siegel-Eisenstein series on $G U(\mathbf{2}, \mathbf{2})$.}

5.2.1. Let $\mathbf{G}=G U(\mathbf{W})$ denote the groups of unitary $\mathcal{F}$-similitude associated to $\underline{\mathbf{W}}$. The matrix of $\boldsymbol{\eta}_{2}=\vartheta_{2,1} \oplus(-\vartheta)$ with respect to the basis $\left\{\mathbf{y}^{i}, \mathbf{x}^{i}\right\}_{i=1,2}$ in (2.13) is $\left[\begin{array}{ll}\mathbf{1}_{2} & -\mathbf{1}_{2}\end{array}\right]$. For an $\mathcal{F}$-algebra $R$, we identify $\mathbf{G}(R)$ with the matrix group

$$
\mathbf{G}(R)=\left\{g \in M_{4}\left(R \otimes_{\mathcal{F}} \mathcal{K}\right) \mid g\left[\begin{array}{ll}
\mathbf{1}_{2} & -\mathbf{1}_{2}
\end{array}\right] g^{*}=\nu(g)\left[\begin{array}{ll}
\mathbf{1}_{2} & -\mathbf{1}_{2}
\end{array}\right]\right\} .
$$

Thus $\mathbf{G}$ is the standard quasi-split group of unitary $\mathcal{F}$-similitude of degree two. Let $Q$ be the stabilizer of the flag $\{0\} \subset \sum_{i=1,2} \mathcal{K} \mathbf{x}^{i} \subset \mathbf{W}$ in $\mathbf{G}$. The unipotent radical $N_{Q}$ of $Q$ and the standard Levi subgroup $M_{Q}$ are given by

$$
N_{Q}=\left\{\mathbf{n}(X):=\left[\begin{array}{ll}
\mathbf{1}_{2} & X \\
& \mathbf{1}_{2}
\end{array}\right] \mid X \in \mathcal{H}_{2}(\mathcal{F})\right\}
$$

and

$$
M_{Q}=\left\{\mathbf{m}(A, \nu):=\left[\begin{array}{ll}
\nu A^{-*} & \\
& A
\end{array}\right] \mid A \in \mathrm{GL}_{2}(\mathcal{K}), \nu \in \mathcal{F}^{\times}\right\} .
$$

The Hermitian symmetric domain associated to $\mathbf{G}$ is $X_{2,2}$ defined in 2.4.1. We choose a distinguished point $\mathbf{i}=\left(\mathbf{i}_{\sigma}\right)_{\sigma \in \Sigma}$ in $X_{2,2}$, where

$$
\mathbf{i}_{\sigma}=\left[\begin{array}{ll}
\sqrt{-1} & \\
& -\frac{\sigma(\vartheta)}{2}
\end{array}\right] \text {. }
$$

Put

$$
\mathbf{K}_{\infty}^{0}=\left\{g \in \mathbf{G}\left(\mathcal{F} \otimes_{\mathbf{Q}} \mathbf{R}\right) \mid g \mathbf{i}=g\right\} .
$$

Let $\mathbf{L}^{s t}$ be the $\mathcal{O}_{\mathcal{K}}$-lattice $\sum_{i=1,2} \mathcal{O}_{\mathcal{K}} \mathbf{y}^{i} \oplus \mathcal{D}_{\mathcal{K} / \mathcal{F}}^{-1} \vartheta \mathbf{x}^{i}$. For $v \in \mathbf{h}$, let $\mathbf{K}_{v}^{0}\left(\right.$ resp. $\left.\mathbf{K}_{v}^{s t}\right)$ be the stabilizer of $\mathbf{L}_{v}$ (resp. $\left.\mathbf{L}_{v}^{s t}\right)$ in $\mathbf{G}\left(\mathcal{F}_{v}\right)$ and put

$$
D_{\mathbf{W}}\left[\mathfrak{n}_{v}\right]=\left\{x \in \mathrm{GL}_{4}\left(\mathcal{K}_{v}\right) \mid \operatorname{det} x \in \mathfrak{r}^{\times}, x \prec\left[\begin{array}{cc}
\mathfrak{r}^{\times} & \mathfrak{r} \\
\mathfrak{n}_{v} & \mathfrak{r}^{\times}
\end{array}\right]\right\}
$$


and $D_{\mathbf{W}}\left(\mathfrak{n}_{v}\right)=D_{\mathbf{W}}\left[\mathfrak{n}_{v}\right] \cap \mathbf{G}\left(\mathcal{F}_{v}\right)$. Define an open-compact subgroup $\mathbf{K}$ of $\mathbf{G}\left(\mathbf{A}_{f}\right)$ by

$$
\mathbf{K}=\prod_{v \mid \mathfrak{n}} D_{\mathbf{W}}\left(\mathfrak{n}_{v}\right) \times \prod_{v \nmid \mathfrak{n}} \mathbf{K}_{v}^{0}
$$

5.2.2. Let $\chi: \mathbf{A}_{\mathcal{K}}^{\times} \rightarrow \mathbf{C}^{\times}$be a Hecke character of $\mathcal{K}^{\times}$of infinity type $k \Sigma$ and let $\chi_{+}:=\left.\chi\right|_{\mathbf{A}^{\times}}$be the restriction of $\chi$ to $\mathbf{A}^{\times}$. We suppose that $k>4$ and $\mathfrak{c}$ is divisible by the prime-to- $p$ conductor $\mathfrak{c}(\chi)$ of $\chi$. For each place $v$ of $\mathcal{F}$ and $s \in \mathbf{C}$, let $I_{v}(\chi, s)$ denote the space of smooth and $\mathbf{K}_{v}^{0}$-finite functions $\phi: \mathbf{G}\left(\mathcal{F}_{v}\right) \rightarrow \mathbf{C}$ such that

$$
\phi(\mathbf{n}(X) \mathbf{m}(A, \nu) g)=\chi_{v}^{-1}(\operatorname{det} A)\left|\nu^{-n} \operatorname{det}\left(A^{*} A\right)\right|^{-s} \phi(g) .
$$

Let $I(\chi, s)=\otimes_{v}^{\prime} I_{v}(\chi, s)$. Conventionally, functions in $I(\chi, s)$ are called sections. The adelic Siegel-Eisenstein series $E_{\mathbf{A}}(g, \phi)$ associated to a section $\phi \in I(\chi, s)$ is defined by

$$
E_{\mathbf{A}}(g, \phi)=\sum_{\gamma \in Q(\mathcal{F}) \backslash \mathbf{G}(\mathcal{F})} \phi(\gamma g) .
$$

It is known that the series $E_{\mathbf{A}}(g, \phi)$ converges absolutely if Re $s$ is large enough.

5.2.3. Fourier coefficients. The Siegel-Eisenstein series $E_{\mathbf{A}}=E_{\mathbf{A}}(g, \phi)$ has the Fourier expansion

$$
E_{\mathbf{A}}(g, \phi)=\sum_{\beta \in \mathcal{H}_{2}(\mathcal{F})} W_{\beta}\left(g, E_{\mathbf{A}}\right),
$$

where $W_{\beta}\left(g, E_{\mathbf{A}}\right)$ is the $\beta$-th Fourier coefficient of $E_{\mathbf{A}}$ given by

$$
W_{\beta}\left(g, E_{\mathbf{A}}\right)=\int_{\mathcal{H}_{2}(\mathcal{F}) \backslash \mathcal{H}_{2}(\mathbf{A})} E_{\mathbf{A}}(\mathbf{n}(X) g) \boldsymbol{\psi}_{-\beta}(X) d X .
$$

Let $\mathbf{w}_{l}=\left[\begin{array}{ll}\mathbf{1}_{2} & -\mathbf{1}_{2}\end{array}\right]$. It is well known that if $\phi=\otimes_{v} \phi_{v}$ and $\operatorname{supp} \phi_{v_{0}} \subset Q\left(\mathcal{F}_{v_{0}}\right)$ $\mathbf{w}_{l} Q\left(\mathcal{F}_{v_{0}}\right)$ for some $v_{0}$, the $\beta$-th Fourier coefficient $W_{\beta}\left(g, E_{\mathbf{A}}\right)$ for $g \in \mathbf{G}\left(\mathbf{A}^{\left(v_{0}\right)}\right)$ is decomposed into a product of local Whittaker integrals $W_{\beta}\left(g_{v}, \phi_{v}\right)$ (for example, see the proof of [Shi97, (18.10.3)] based on Lemma 18.8 loc. cit.). In other words, we have

$$
\begin{aligned}
& W_{\beta}\left(g, E_{\mathbf{A}}\right)=c_{2}(\mathcal{K}) \cdot \prod_{v} W_{\beta}\left(g_{v}, \phi_{v}\right), \text { where } \\
& W_{\beta}\left(g_{v}, \phi_{v}\right)=\int_{\mathcal{H}_{2}\left(\mathcal{F}_{v}\right)} \phi_{v}\left(\mathbf{w}_{l} \mathbf{n}\left(X_{v}\right) g_{v}\right) \boldsymbol{\psi}_{\beta}\left(X_{v}\right) d X_{v} .
\end{aligned}
$$

5.3. The choice of local sections. To define the desired Eisenstein series with nice $p$-adic properties, we need to make a special choice of local sections in $I_{v}(\chi, s)$ at each place $v$. In this subsection, we give the recipe of choices of these local sections. Let

$$
S=\{v \in \mathbf{h}|v| \mathfrak{n}\} \text { be a non-empty set. }
$$

Then $\chi$ and $\mathcal{K} / \mathcal{F}$ are unramified outside $S \cup S_{p}$. 
5.3.1. The local sections at archimedean places. For $g=\left(g_{\sigma}\right)_{\sigma \in \mathbf{a}} \in \mathbf{G}\left(\mathcal{F} \otimes_{\mathbf{Q}} \mathbf{R}\right)=$ $\prod_{\sigma \in \mathbf{a}} \mathbf{G}(\mathbf{R})$ and $Z=\left(Z_{\sigma}\right)_{\sigma \in \mathbf{a}} \in X_{2,2}$, we define two standard automorphy factors $\mathbf{J}(g, Z)$ and $\mathbf{J}^{\prime}(g, Z)$ by

$$
\mathbf{J}(g, Z)=\prod_{\sigma \in \mathbf{a}} \mathbf{J}_{\sigma}(g, Z) \text { and } \mathbf{J}^{\prime}(g, Z)=\prod_{\sigma \in \mathbf{a}} \operatorname{det}\left(g_{\sigma}\right)^{-1} \mathbf{J}_{\sigma}(g, Z) \nu\left(g_{\sigma}\right)^{2},
$$

where $\mathbf{J}_{\sigma}(g, Z)=\operatorname{det}\left(c_{g_{\sigma}} Z_{\sigma}+d_{g_{\sigma}}\right)$. Denote by $\mathbf{J}_{(\mu, \lambda)}(g, Z)$ the automorphy factor of weight $(\mu, \lambda) \in \mathbf{Z}^{2}$ given by

$$
\mathbf{J}_{(\mu, \lambda)}(g, Z)=\mathbf{J}^{\prime}(g, Z)^{\mu} \mathbf{J}(g, Z)^{\lambda} .
$$

We define the section $\phi_{k, \infty}$ of minimal $\mathbf{K}_{\infty}$-type $(0, k)$ in $I_{\infty}(\chi, 0)$ by

$$
\phi_{k, \infty}(g)=\mathbf{J}_{(0, k)}(g, Z)=\mathbf{J}(g, \mathbf{i})^{-k}
$$

and set

$$
\phi_{k, s, \infty}(g)=\phi_{k, \infty}(g) \cdot|\mathbf{J}(g, \mathbf{i}) \overline{\mathbf{J}(g, \mathbf{i})}|^{-s} \cdot|\operatorname{det} g|^{s} \in I_{\infty}(\chi, s) .
$$

5.3.2. The local sections at unramified places. Fix $\boldsymbol{\xi}=\left(\boldsymbol{\xi}_{v}\right) \in U(\mathbf{W})\left(\mathbf{A}_{f}\right)$ such that $\mathbf{L}_{v} \boldsymbol{\xi}_{v}=\mathbf{L}_{v}^{s t}$ if $v \notin S$ and $\boldsymbol{\xi}_{v}=1$ if $v \in S$. Since the lattices $\mathbf{L}_{v}$ and $\mathbf{L}_{v}^{s t}$ are $\mathcal{O}_{\mathcal{F}_{v}}$-maximal $\mathcal{O}_{\mathcal{K}_{v}}$-lattices with respect to $\vartheta^{-1} \boldsymbol{\eta}_{2}$ for each finite place $v \nmid D_{\mathcal{K} / \mathcal{F}}$, the existence of $\boldsymbol{\xi}$ follows from [Shi97, Lemma 5.9]. If $v \notin S$ is a finite place where $\chi_{v}$ is unramified, we let $f_{\chi, s, v}^{s t}$ be the section in $I_{v}(\chi, s)$ such that $f_{\chi, 0, v}^{s t}\left(\mathbf{K}_{v}^{s t}\right)=1$ and let

$$
f_{\chi, s, v}^{0}(g):=f_{\chi, s, v}^{s t}\left(g \boldsymbol{\xi}_{v}\right) .
$$

5.3.3. The local sections at $v \in S$. For $v \in S$, we let $f_{\mathfrak{n}, v}$ be the unique section in $I_{v}(\chi, s)$ such that

$$
\operatorname{supp} f_{\mathfrak{n}, v}=Q\left(\mathcal{F}_{v}\right) D_{\mathbf{W}}\left(\mathfrak{n}_{v}\right) \text { and } f_{\mathfrak{n}, v}(u)=\chi_{v}^{-1}\left(d_{u}\right), u \in D_{\mathbf{W}}\left(\mathfrak{n}_{v}\right) .
$$

Let $\mathbf{u}=\left[\begin{array}{ll}-1 & \\ & -\frac{\vartheta}{2}\end{array}\right]$. Put

$$
f_{\mathfrak{n}, v}^{1}(g):=f_{\mathfrak{n}, v}\left(g \mathbf{w}_{l}^{\prime}\right), \mathbf{w}_{l}^{\prime}=\left[\begin{array}{ll}
\mathbf{u} \\
-\mathbf{u}^{-*}
\end{array}\right] \in \mathbf{G}\left(\mathcal{F}_{v}\right) .
$$

Define $\mathcal{L}_{v}=\mathcal{H}_{2}\left(\mathcal{F}_{v}\right) \cap\left(\mathbf{u} M_{2}\left(\mathfrak{n}_{v}\right) \mathbf{u}^{*}\right)$ to be a lattice in $\mathcal{H}_{2}\left(\mathcal{F}_{v}\right)$. One checks immediately that $f_{\mathfrak{n}, v}^{1}$ is the unique section such that

$$
\begin{aligned}
\operatorname{supp} f_{\mathfrak{n}, v}^{1} & =Q\left(\mathcal{F}_{v}\right) \mathbf{w}_{l} \mathbf{n}\left(\mathcal{L}_{v}\right) \subset Q\left(\mathcal{F}_{v}\right) \mathbf{w}_{l} N_{Q}\left(\mathcal{F}_{v}\right), \\
f_{\mathfrak{n}, v}^{1}\left(\mathbf{w}_{l} \mathbf{n}\left(X_{v}\right)\right) & =\chi_{v}^{-1}(\operatorname{det} \mathbf{u})|\operatorname{det}(\mathbf{u} \overline{\mathbf{u}})|^{-s} \text { for all } X_{v} \in \mathcal{L}_{v} .
\end{aligned}
$$

In particular, $f_{\mathfrak{n}, v}^{1}$ is supported in the big cell.

5.3.4. The local sections at $v \in S_{p}$. We begin with some notation. Write $v=w \bar{w}$ with $w \in \Sigma_{p}$ and $\chi_{v}=\left(\chi_{w}, \chi_{\bar{w}}\right)=\left(\chi_{1}, \chi_{2}\right)$. Let $F=\mathcal{F}_{v}$. With the identification $\mathbf{G}(F) \stackrel{\sim}{\rightarrow} \mathrm{GL}_{4}(F) \times F^{\times}$in (1.2), we obtain an isomorphism $I_{v}(\chi, s) \simeq I\left(\chi_{2}, \chi_{1}^{-1}\right)$ sending $f$ to its restriction to $U(\mathbf{W})(F) \simeq \mathrm{GL}_{4}(F)$, where $I\left(\chi_{1}, \chi_{2}^{-1}\right)$ consists of smooth functions $f: \mathrm{GL}_{4}(F) \rightarrow \mathbf{C}$ such that

$$
f\left(\left[\begin{array}{cc}
A & B \\
0 & D
\end{array}\right] g\right)=\chi_{2}(\operatorname{det} A) \chi_{1}^{-1}(\operatorname{det} D)\left|\operatorname{det} A \operatorname{det} D^{-1}\right|^{s} f(g) .
$$


Fix a uniformizer $\varpi=\varpi_{v}$ of $\mathcal{O}=\mathcal{O}_{F}$. If $\mu: F^{\times} \rightarrow \mathbf{C}^{\times}$is a character of $F^{\times}$, we let $\varphi_{\mu}$ be the Bruhat-Schwartz function on $F$ given by $\varphi_{\mu}(x)=\mu(x) \mathbb{I}_{\mathcal{O} \times}(x)$ and let $\widehat{\varphi}_{\mu}$ be the Fourier transform of $\varphi_{\mu}$. Let $\left(\varpi^{a(\mu)}\right)$ be the conductor of $\mu$. A direct computation shows that

$$
\widehat{\varphi}_{\mu}(x)= \begin{cases}\mathbb{I}_{\mathcal{O}}(x)-|\varpi| \mathbb{I}_{\varpi^{-1} \mathcal{O}}(x) & \text { if } \mu \text { is unramified, } \\ \mu^{-1}(x) \mathbb{I}_{c^{-1} \mathcal{O} \times}(x) \cdot \mathfrak{g}(\mu) & \text { if } \mu \text { is ramified, }\end{cases}
$$

where $\mathfrak{g}(\mu)$ is the Gauss sum defined by

$$
\mathfrak{g}(\mu)=\int_{\mathcal{O} \times} \mu\left(\frac{x}{\varpi^{a(\mu)}}\right) \psi_{v}\left(\frac{x}{\varpi^{a(\mu)}}\right) d x
$$

Definition 5.1 (Modified $p$-Euler factor). Put

$$
\operatorname{Eul}(s, \mu):=Z\left(s, \mu, \widehat{\varphi}_{\mu}\right)=\int_{F} \mu(x)|x|^{s} \widehat{\varphi}_{\mu}(x) d^{\times} x .
$$

By Tate's local functional equation, we find that

$$
\operatorname{Eul}(s, \mu)=\frac{L(s, \mu)}{L\left(1-s, \mu^{-1}\right) \epsilon(s, \mu)},
$$

where $\epsilon(s, \mu)$ is Tate's epsilon factor with respect to the additive character $x \mapsto$ $\psi_{v}(-x)$.

We introduce two special Bruhat-Schwartz functions $\Phi_{\bar{w}}$ and $\Phi_{w}$ on $M_{2}(F)$ as follows. Put

$$
\mu_{2}=\chi_{2}^{-1}, \nu_{1}=\chi_{1} \chi_{2}, \nu_{2}=\chi_{1}
$$

and define two subsets $\mathcal{P}_{1, v}$ and $\mathcal{P}_{2, v}$ of $M_{2}(F)$ by

$$
\mathcal{P}_{1, v}=\left[\begin{array}{cc}
\varpi \mathcal{O} & \mathcal{O} \\
\varpi \mathcal{O}^{\times} & \mathcal{O}^{\times}
\end{array}\right] \text {and } \mathcal{P}_{2, v}=\left[\begin{array}{cc}
\mathcal{O}^{\times} & \varpi^{m_{v}} \mathcal{O} \\
\mathcal{O} & \mathcal{O}^{\times}
\end{array}\right], m_{v}=\max \left\{1, a\left(\chi_{1}\right), a\left(\chi_{2}\right)\right\}
$$

We set

$$
\begin{aligned}
& \Phi_{\bar{w}}(X)=\mathbb{I}_{\mathcal{P}_{1, v}}(X) \varphi_{\mu_{2}}\left(\varpi^{-1} X_{3}\right) \quad\left(X=\left[\begin{array}{cc}
X_{1} & X_{2} \\
X_{3} & X_{4}
\end{array}\right]\right), \\
& \Phi_{w}(X)=\mathbb{I}_{\mathcal{P}_{2, v}}(X) \varphi_{\nu_{1}}\left(X_{1}\right) \varphi_{\nu_{2}}\left(X_{4}\right) .
\end{aligned}
$$

Define the Bruhat-Schwartz function $\Phi_{v}$ on $M_{2 \times 4}(F)$ by

$$
\Phi_{v}(X, Y)=\Phi_{\bar{w}}(X) \otimes \widehat{\Phi}_{w}(Y) \quad\left(X, Y \in M_{2}(F)\right),
$$

where $\widehat{\Phi}_{w}$ is the Fourier transformation of $\Phi_{w}$ defined by

$$
\widehat{\Phi}_{w}(Y)=\int_{M_{2}(F)} \Phi_{w}(Z) \boldsymbol{\psi}_{v}\left(\operatorname{Tr}\left(Z^{t} Y\right)\right) d Z
$$

We let $\mathrm{GL}_{4}(F)$ act on $\Phi_{v}$ by $g . \Phi_{v}(X, Y)=\Phi_{v}((X, Y) g)$.

Lemma 5.2. The functions $\Phi_{v}, \Phi_{w}$ and $\Phi_{\bar{w}}$ enjoy the following properties:

(1) For $t=\left[\begin{array}{ll}t_{1} & \\ & t_{2}\end{array}\right] \in T_{2}(\mathcal{O}), \Phi_{\bar{w}}(X t)=\chi_{2}^{-1}\left(t_{1}\right) \Phi_{\bar{w}}(X)$ and $\widehat{\Phi}_{w}(X t)=$ $\chi_{1}^{-1} \chi_{2}^{-1}\left(t_{1}\right) \chi_{1}^{-1}\left(t_{2}\right) \widehat{\Phi}_{w}(X)$.

(2) $\Phi_{v}$ is $N_{4}(\mathcal{O})$-invariant. 
Proof. Part (1) immediately follows from the definitions of $\Phi_{\bar{w}}$ and $\Phi_{w}$. Part (2) can be deduced easily from the following observations:

(i) For $A \in N_{2}(\mathcal{O}), \mathcal{P}_{1, v} A=\mathcal{P}_{1, v}, \Phi_{\bar{w}}(X A)=\Phi_{\bar{w}}(X)$ and $\Phi_{w}\left(Y A^{t}\right)=\Phi_{w}(Y)$.

(ii) $\widehat{\Phi}_{w}\left(Y+M_{2}(\mathcal{O})\right)=\widehat{\Phi}_{w}(Y)$.

We define the local section at $v \mid p$ to be the Godement-Jacquet section $f_{\Phi_{v}, v}$ associated to $\Phi_{v}$. Namely,

$$
f_{\Phi_{v}, v}(g):=\chi_{2}(\operatorname{det} g)|\operatorname{det} g|^{s} \int_{\mathrm{GL}_{2}(F)} \Phi_{v}((0, Z) g) \chi_{1} \chi_{2}(\operatorname{det} Z)|\operatorname{det} Z|^{2 s} d^{\times} Z
$$

where $d^{\times} Z$ is the Haar measure with $\operatorname{vol}\left(\mathrm{GL}_{2}(\mathcal{O}), d^{\times} Z\right)=1$. It is easy to verify that $f_{\Phi_{v}, v}$ converges absolutely for $\operatorname{Re} s>\frac{1-k}{2}$ and $f_{\Phi_{v}, v} \in I\left(\chi_{2}, \chi_{1}^{-1}\right)=I_{v}(\chi, s)$. For $t=\operatorname{diag}\left(t_{1}, t_{2}, t_{3}, t_{4}\right) \in T_{4}(\mathcal{O})$ and $f \in I_{v}(\chi, s)$, we define

$$
[t] \cdot f(g)=f\left(g \cdot \operatorname{diag}\left(t_{1}, t_{2}, t_{3}^{-1}, t_{4}^{-1}\right)\right) .
$$

Lemma 5.3. We have

(1) $[t] . f_{\Phi_{v}, v}=\left(\mathbf{1}, \chi_{2}, \chi_{1}, \chi_{1} \chi_{2}^{-1}\right)(t) \cdot f_{\Phi_{v}, v}$ for $t \in T_{4}(\mathcal{O})$.

(2) $f_{\Phi_{v}, v}$ is right invariant by the open-compact subgroup

$$
I_{1}\left(p^{n}\right)_{v}=\left\{(g, a) \in \mathrm{GL}_{4}(\mathcal{O}) \times \mathcal{O}^{\times} \mid g \in N_{4}(\mathcal{O})\left(\bmod p^{n}\right), a \equiv 1\left(\bmod p^{n}\right)\right\}
$$

for sufficiently large $n>\max \left\{a\left(\chi_{1}\right), a\left(\chi_{2}\right)\right\}$.

Proof. This is a consequence of the observation $f_{\Phi_{v}, v}(h g)=\chi_{2}(\operatorname{det} g)|\operatorname{det} g|^{s}$. $f_{g . \Phi_{v}, v}(h)$ combined with Lemma 5.2 .

5.3.5. Local Whittaker integrals. In the following proposition, we summarize the formulas of local Whittaker integrals of our sections.

Proposition 5.4. Let $\beta \in \mathcal{H}_{2}(\mathcal{F})$.

(1) If $\sigma \in \Sigma$ be an archimedean place, put

$$
\Lambda_{2, \sigma}(s, \chi)=2^{-1}(-1)^{k} \Gamma_{\mathbf{C}}(s+k) \Gamma_{\mathbf{C}}(s+k-1) \quad\left(\Gamma_{\mathbf{C}}(s)=2(2 \pi)^{-s} \Gamma(s)\right),
$$

and we have

$$
\begin{aligned}
& \left.\mathbf{J}_{\sigma}\left(g_{\sigma}, \mathbf{i}_{\sigma}\right)^{k} W_{\beta}\left(g_{\sigma}, \phi_{\chi, s, \sigma}\right)\right|_{s=0} \\
& =\left\{\begin{array}{lll}
\Lambda_{2, \sigma}(0, \chi)^{-1} \cdot(\operatorname{det} \sigma(\beta))^{k-2} \cdot e^{2 \pi i \operatorname{Tr}\left(\sigma(\beta) Z_{\sigma}\right)} & \left(Z_{\sigma}=g_{\sigma} \mathbf{i}_{\sigma}\right), & \sigma(\beta) \geq 0, \\
0, & \text { otherwise. }
\end{array}\right.
\end{aligned}
$$

(2) If $v \notin S \cup S_{p}$ is a finite place and $\operatorname{det} \beta \neq 0$, put

$$
\Lambda_{2, v}(s, \chi)=L\left(2 s, \chi_{+, v}\right) L\left(2 s-1, \chi_{+, v} \tau_{\mathcal{K}_{v} / \mathcal{F}_{v}}\right),
$$

and we have

$$
\begin{aligned}
W_{\beta}\left(\boldsymbol{\xi}_{v}^{-1}, f_{\chi, s, v}^{0}\right) & =W_{\beta}\left(1, f_{\chi, s, v}^{s t}\right) \\
& =\left|\mathfrak{b}_{\vartheta}\right|_{v}^{2 s+4} \Lambda_{2, v}(s, \chi)^{-1} R_{\beta, v}\left(\chi_{+, v}\left(\varpi_{v}\right)\left|\varpi_{v}\right|^{2 s}\right) \mathbb{I}_{\mathcal{H}_{2}\left(\mathfrak{b}_{\vartheta, v}\right)^{\vee}}(\beta),
\end{aligned}
$$

where $R_{\beta, v}(T) \in \mathbf{Z}[T]$ is a polynomial which only depends on $\beta$ and is equal to 1 for all but finitely many $v$.

(3) If $v \in S$, then

$$
W_{\beta}\left(1, f_{\mathfrak{n}, v}^{1}\right)=\chi^{-1}(\operatorname{det} \mathbf{u})|\operatorname{det} \mathbf{u} \overline{\mathbf{u}}|_{v}^{-2 s} \mathbb{I}_{\mathcal{L}_{v}^{v}}(\beta) \operatorname{vol}\left(\mathcal{L}_{v}, d X_{v}\right),
$$

where $\mathcal{L}_{v}^{\vee}$ is the dual lattice defined in (5.1). 
(4) If $v \in S_{p}$, then

$$
W_{\beta}\left(1, f_{\Phi_{v}, v}\right)= \begin{cases}\operatorname{vol}\left(\mathcal{P}_{2, v}\right) \chi_{+, v}(\operatorname{det} \beta)|\operatorname{det} \beta|_{v}^{2 s-2} \Phi_{\bar{w}}(\beta), & \operatorname{det} \beta \neq 0, \\ 0, & \operatorname{det} \beta=0 .\end{cases}
$$

(5) If $v \in S_{p}$, $\operatorname{det} \beta \neq 0$ and $\chi_{v}$ is unramified, then

$$
W_{\beta}\left(1, f_{\chi, s, v}^{s t}\right)=\Lambda_{2, v}(s, \chi)^{-1} \cdot R_{\beta, v}^{*}\left(\chi_{+, v}|\cdot|^{2 s-2}\left(\varpi_{v}\right)\right) \mathbb{I}_{M_{2}\left(\mathcal{O}_{\mathcal{F}_{v}}\right)}(\beta),
$$

where $R_{\beta, v}^{*}(T) \in \mathbf{Z}[T]$ is a polynomial of degree no greater than $v(\operatorname{det} \beta)$ and only depends on the coset $\mathrm{GL}_{2}\left(\mathcal{O}_{\mathcal{F}_{v}}\right) \beta \mathrm{GL}_{2}\left(\mathcal{O}_{\mathcal{F}_{v}}\right)$.

Proof. The formulas (1) and (2) of local Whittaker integrals at archimedean and unramified places are due to Shimura. For (2), it is proved in Shi97, Prop. 18.14, Prop. 19.2]. For (1), we recall that if $Z_{\sigma}=X+i Y$, then

$$
\begin{aligned}
\mathbf{J}_{\sigma}\left(g_{\sigma}, \mathbf{i}_{\sigma}\right)^{k} W_{\beta}\left(g_{\sigma}, \phi_{\chi, s, \sigma}\right)= & (\operatorname{det} Y)^{2-k-s} \operatorname{det}(-i \vartheta / 2)^{s} \exp (-\operatorname{Tr}(\sigma(\beta) X)) \\
& \cdot \xi(1, Y \beta, k+s, s),
\end{aligned}
$$

where $\xi$ is the four-variable function defined in Shi83, (3.18), page 432] (cf. [Hsi11, page 1016]). Then (1) follows from the evaluation formulas of $\xi$ in Shi83, page 457 (7.11), (7.12)]. The equation (3) follows from (5.5) and a straightforward computation

$$
\begin{aligned}
W_{\beta}\left(1, f_{\mathfrak{n}, v}^{1}\right) & =\int_{\mathcal{H}_{2}\left(\mathcal{F}_{v}\right)} f_{\mathfrak{n}, v}^{1}\left(\mathbf{w}_{l} \mathbf{n}\left(X_{v}\right)\right) \boldsymbol{\psi}_{-\beta}\left(X_{v}\right) d X_{v} \\
& =\chi^{-1}(\operatorname{det} \mathbf{u})|\operatorname{det} \mathbf{u} \overline{\mathbf{u}}|_{v}^{-2 s} \int_{\mathcal{H}_{2}\left(\mathcal{F}_{v}\right)} \mathbb{I}_{\mathcal{L}}\left(X_{v}\right) \boldsymbol{\psi}_{-\beta}\left(X_{v}\right) d X_{v} \\
& =\chi^{-1}(\operatorname{det} \mathbf{u})|\operatorname{det} \mathbf{u} \overline{\mathbf{u}}|_{v}^{-2 s} \mathbb{I}_{\mathcal{L}_{v}^{v}}(\beta) \operatorname{vol}\left(\mathcal{L}_{v}, d X_{v}\right) .
\end{aligned}
$$

We proceed to prove (4). Let $v \in S_{p}$. Let $F=\mathcal{F}_{v}, \mathcal{O}=\mathcal{O}_{\mathcal{F}_{v}}$ and $\varpi=\varpi_{v}$. By definition,

$$
\begin{aligned}
W_{\beta}\left(1, f_{\Phi_{v}, v}\right)= & \int_{M_{2}(F)} \int_{\mathrm{GL}_{2}(F)} \Phi_{v}\left((0, Z)\left[\begin{array}{cc}
0 & -1 \\
1 & X
\end{array}\right]\right) \\
& \times \chi_{+, v}(\operatorname{det} Z)|\operatorname{det} Z|^{2 s} d^{\times} Z \boldsymbol{\psi}_{-\beta}(X) d X \\
= & \int_{\mathrm{GL}_{2}(F)} \Phi_{\bar{w}}(Z) \Phi_{w}\left({ }^{t} Z^{-1 t} \beta\right) \chi_{+, v}(\operatorname{det} Z)|\operatorname{det} Z|^{2 s-2} d^{\times} Z .
\end{aligned}
$$

Recall that $\chi_{+, v}=\chi_{w} \chi_{\bar{w}}$. Since $\Phi_{w}$ is supported in non-degenerate matrices, $W_{\beta}\left(1, f_{\Phi_{v}, v}\right)=0$ if $\operatorname{det} \beta=0$. Suppose that $\operatorname{det} \beta \neq 0$. Making a change of variable $Z \mapsto Z \beta$, we find that

$$
\begin{aligned}
W_{\beta}\left(1, f_{\Phi_{v}, v}\right)= & \chi_{+, v}(\operatorname{det} \beta)|\operatorname{det} \beta|^{2 s-2} \\
& \times \int_{\mathrm{GL}_{2}(F)} \Phi_{\bar{w}}(Z \beta) \Phi_{w}\left({ }^{t} Z^{-1}\right) \chi_{+, v}(\operatorname{det} Z)|\operatorname{det} Z|^{2 s} d^{\times} Z \\
= & \chi_{+, v}(\operatorname{det} \beta)|\operatorname{det} \beta|^{2 s-2} \int_{\mathcal{P}_{2, v}} \Phi_{\bar{w}}(Z \beta) \Phi_{w}\left({ }^{t} Z^{-1}\right) \chi_{+, v}(\operatorname{det} Z) d^{\times} Z \\
= & \operatorname{vol}\left(\mathcal{P}_{2, v}\right) \chi_{+, v}(\operatorname{det} \beta)|\operatorname{det} \beta|^{2 s-2} \Phi_{\bar{w}}(\beta) .
\end{aligned}
$$


This completes the proof of (4). To prove (5), we introduce the spherical Godement section

$$
f_{\Phi^{0}, v}(g):=\chi_{2}(\operatorname{det} g)|\operatorname{det} g|^{s} \int_{\mathrm{GL}_{2}(F)} \Phi^{0} \otimes \Phi^{0}((0, Z) g) \chi_{1} \chi_{2}(\operatorname{det} Z)|\operatorname{det} Z|^{2 s} d^{\times} Z,
$$

where $\Phi^{0}$ is the characteristic function of $M_{2}(\mathcal{O})$. Then $f_{\Phi^{0}, v}$ is a spherical vector, and it is known that

$$
f_{\Phi^{0}, v}=L\left(2 s, \chi_{+, v}\right) L\left(2 s-1, \chi_{+, v}\right) \cdot f_{\chi, s, v}^{s t}=\Lambda_{2, v}(s, \chi) \cdot f_{\chi, s, v}^{s t} .
$$

It suffices to compute $W_{\beta}\left(1, f_{\Phi^{0}, v}\right)$. Let $K=\mathrm{GL}_{2}(\mathcal{O})$. For $\left(a_{1}, a_{2}\right) \in \mathbf{Z}^{2}$, define the coset

$$
C\left(a_{1}, a_{2}\right):=\bigsqcup_{y \in \mathcal{O} /\left(\varpi^{a_{2}}\right)} K\left[\begin{array}{cc}
\varpi^{a_{1}} & y \\
0 & \varpi^{a_{2}}
\end{array}\right]
$$

We have $\widehat{\Phi}^{0}=\Phi^{0}$ and

$$
\begin{aligned}
W_{\beta}\left(1, f_{\Phi^{0}, v}\right) & =\int_{\mathrm{GL}_{2}(F)} \Phi^{0}(Z) \widehat{\Phi}^{0}\left({ }^{t} Z^{-1 t} \beta\right) \chi_{+, v}(\operatorname{det} Z)|\operatorname{det} Z|^{2 s-2} d^{\times} Z \\
& =\sum_{\left(a_{1}, a_{2}\right) \in \mathbf{Z}_{\geq 0}^{2}} \chi_{+, v}|\cdot|^{2 s-2}\left(\varpi^{a_{1}+a_{2}}\right) \int_{C\left(a_{1}, a_{2}\right)} \Phi^{0}\left(\beta Z^{-1}\right) d^{\times} Z .
\end{aligned}
$$

Hence $W_{\beta}\left(1, f_{\Phi^{0}, v}\right)=0$ unless $\beta \notin M_{2}(\mathcal{O})$. Suppose that $\beta \in C\left(b_{1}, b_{2}\right)$. then

Note that if $Z \in K\left[\begin{array}{cc}x_{1} & y \\ 0 & x_{2}\end{array}\right]$ with $Z \in M_{2}(\mathcal{O})$ and $\left[\begin{array}{cc}\varpi^{b_{1}} & 0 \\ 0 & \varpi^{b_{2}}\end{array}\right] Z^{-1} \in M_{2}(\mathcal{O})$,

$$
x_{1}, x_{2}, y \in \mathcal{O} ; \beta_{1} x_{1}^{-1}, \varpi^{b_{1}} x_{1}^{-1} x_{2}^{-1}, \varpi^{b_{2}} x_{2}^{-1} \in \mathcal{O} .
$$

We find that

$$
\begin{aligned}
\int_{C\left(a_{1}, a_{2}\right)} \Phi^{0}\left(\beta Z^{-1}\right) d^{\times} Z & =\int_{C\left(a_{1}, a_{2}\right)} \Phi^{0}\left(\left[\begin{array}{cc}
\varpi^{b_{1}} & 0 \\
0 & \varpi^{b_{2}}
\end{array}\right] Z^{-1}\right) d^{\times} Z \\
& =\sharp\left(\varpi^{a_{2}+a_{1}-b_{1}} \mathcal{O} \cap \mathcal{O} / \varpi^{a_{2}} \mathcal{O}\right) .
\end{aligned}
$$

Therefore, $W_{\beta}\left(1, f_{\Phi^{0}, v}\right)=R_{\beta, v}^{*}\left(\chi_{+, v}|\cdot|^{2 s-2}(\varpi)\right)$, where

$$
R_{\beta, v}^{*}(T)=\sum_{0 \leq a_{1} \leq b_{1}, 0 \leq a_{2} \leq b_{2}} \sharp\left(\varpi^{a_{2}+a_{1}-b_{1}} \mathcal{O} \cap \mathcal{O} / \varpi^{a_{2}} \mathcal{O}\right) \cdot T^{a_{1}+a_{2}} .
$$

This completes the proof of (5).

Remark 5.5. Note that Shimura considers Eisenstein series constructed by sections attached to unitary Hecke characters (see [Shi97, (18.4.6)]). At archimedean and unramified places, our local sections restricted to $U(2,2)$ are indeed Shimura's sections attached to $\chi^{*}:=\chi|\cdot|_{\mathbf{A}_{\mathcal{K}}}^{-\frac{k}{2}}\left[\right.$ Shi97, (18.6.3)] multiplied by the function $\varepsilon^{-\frac{k}{2}}$ defined in [Shi97, §18.4], and Shimura's section evaluated at $k / 2$ is the same with ours evaluated at 0 . 


\subsection{Normalized Siegel-Eisenstein series.}

Definition 5.6. Define the section $\phi_{\chi, s}=\otimes \phi_{\chi, s, v} \in I(\chi, s)$ by

$$
\phi_{\chi, s}=\phi_{k, s, \infty} \bigotimes_{v \notin S \cup S_{p}} f_{\chi, s, v}^{0} \bigotimes_{v \in S} f_{\mathfrak{n}, v}^{1} \bigotimes_{v \in S_{p}} \frac{1}{\operatorname{vol}\left(\mathcal{P}_{2, v}\right)} \cdot f_{\Phi_{v}, v}
$$

Define the normalized section $\phi_{\chi, s}^{*}:=\Lambda_{2}^{S \cup S_{p}}(\chi, s) \cdot \phi_{\chi, s}$, where

$$
\Lambda_{2}^{S \cup S_{p}}(\chi, s):=\prod_{v \notin S \cup S_{p}} \Lambda_{2, v}(s, \chi)=L^{S \cup S_{p}}\left(2 s, \chi_{+}\right) \cdot L^{S \cup S_{p}}\left(2 s-1, \chi_{+} \tau_{\mathcal{K} / \mathcal{F}}\right) .
$$

The adelic Siegel-Eisenstein series $E_{\mathbf{A}}\left(g, \phi_{\chi, s}\right)$ converges absolutely for $\operatorname{Re} s>$ $2-\frac{k}{2}$. Since $k>4$, we can define the $\mathbf{C}$-valued function $E(\chi, \mathfrak{n})$ on $X_{2,2} \times \mathbf{G}\left(\mathbf{A}_{f}\right)$ by

$$
\begin{aligned}
E(\chi, \mathfrak{n})\left(Z, g_{f}\right):= & \left.\chi^{3}\left(\nu\left(g_{f}\right)\right) \mathbf{J}_{(0, k)}\left(g_{\infty}, \mathbf{i}\right) E_{\mathbf{A}}\left(\left(g_{\infty}, g_{f}\right), \phi_{\chi, s}^{*}\right)\right|_{s=0} \\
& \left(g_{\infty} \in \mathbf{G}\left(\mathcal{F} \otimes_{\mathbf{Q}} \mathbf{R}\right)^{+}, \quad g_{\infty} \mathbf{i}=Z\right) .
\end{aligned}
$$

Then $E(\chi, \mathfrak{n})$ is a holomorphic Siegel-Eisenstein series on $G U(2,2)$ of weight $k$ (cf. [Shi97, Thm. 19.7]). We claim that $E(\chi, \mathfrak{n})$ is right invariant by $\mathbf{K}_{1}^{n}=$ $\mathbf{K}^{(p)} \prod_{v \mid p} I_{1}\left(p^{n}\right)_{v}$ for some $n$. Indeed, it suffices to verify the right invariance by $\mathbf{K}_{1}^{n}$ of the local section $\phi_{\chi, s, v}$ at all finite places. If $v \notin S_{p}$, it is clear by the definition of local sections at these places. If $v \in S_{p}$, it follows from Lemma 5.3 (2).

Now we calculate the Fourier expansion of $E(\chi, \mathfrak{n})$ at special cusps. For each $\beta \in \mathcal{H}_{2}\left(\mathbf{A}_{f}\right)$ with $\operatorname{det} \beta \neq 0$, we put

$$
\begin{aligned}
\mathbf{a}_{\beta}(\chi, \mathfrak{n}):= & \chi_{p}(\operatorname{det} \beta)|\operatorname{det} \beta|_{p}^{-2} \prod_{v \in S} \chi_{v}\left(\operatorname{det} \mathbf{u}^{-1}\right) \cdot \prod_{\substack{v \notin S \cup S_{p}, v(\operatorname{det} \beta)>0}} R_{\beta, v}\left(\chi_{v}\left(\varpi_{v}\right)\right) \\
& \cdot c_{S}(\mathcal{K}, \mathcal{L}) \cdot \mathbb{I}_{\Xi}(\beta),
\end{aligned}
$$

where $c_{S}(\mathcal{K}, \mathcal{L}) \in \overline{\mathbf{Z}}_{(p)}^{\times}$is a $p$-adic unit given by

$$
c_{S}(\mathcal{K}, \mathcal{L})=c_{2}(\mathcal{K}) \cdot\left|\mathfrak{b}_{\vartheta}\right|_{\mathbf{A}_{f}}^{4} \cdot \prod_{v \in S} \operatorname{vol}\left(\mathcal{L}_{v}, d X_{v}\right),
$$

and $\Xi$ is the compact subset in $\mathcal{H}_{2}\left(\mathbf{A}_{\mathcal{K}, f}\right)$ given by

$$
\Xi=\prod_{v \in S} \mathcal{L}_{v}^{\vee} \times \prod_{v \in S_{p}} \mathcal{P}_{1, v} \times \prod_{v \notin S \cup S_{p}} \mathcal{H}_{2}\left(\mathfrak{b}_{\vartheta, v}\right)^{\vee} .
$$

Note that $\Xi$ is independent of $\chi$.

Proposition 5.7. Let $A \in \mathrm{GL}_{2}\left(\mathbf{A}_{f}^{S \cup S_{p}}\right) \times \mathrm{GL}_{2}\left(\mathcal{O}_{\mathcal{K}} \otimes \mathbf{z} \mathbf{Z}_{p}\right)$ and $g_{A}:=\mathbf{m}(A, 1) \boldsymbol{\xi}^{-1} \in$ $\mathbf{G}\left(\mathbf{A}_{f}^{S}\right)$. Then $E(\chi, \mathfrak{n})\left(Z, g_{A}\right)$ has the Fourier expansion

$$
\sum_{\substack{\left.\beta \in \mathcal{H}_{2}(\mathcal{F}), \beta\right)>0 \text { for all } \sigma \in \Sigma}} \mathbf{a}_{\beta}\left(g_{A}, E(\chi, \mathfrak{n})\right) q^{\beta},
$$

where $q^{\beta}=\exp \left(2 \pi i \sum_{\sigma} \operatorname{Tr}\left(\sigma(\beta) Z_{\sigma}\right)\right)$ and

$$
\mathbf{a}_{\beta}\left(g_{A}, E(\chi, \mathfrak{n})\right)=\chi^{-1}(\operatorname{det} A)|\operatorname{det} A|_{\mathbf{A}_{\mathcal{K}}}^{2} \cdot \mathbf{a}_{A^{*} \beta A}(\chi, \mathfrak{n})(\operatorname{det} \beta)^{(k-2) \Sigma} .
$$


Proof. Since the local sections $\phi_{\chi, s, v}$ for $v \in S$ are supported in the big cell, we have

$$
\mathbf{a}_{\beta}(g, E(\chi, \mathfrak{n}))=\prod_{v} W_{\beta, v}\left(\phi_{\chi, s, v}, g_{v}\right) \text { for each } g \in \mathbf{G}\left(\mathbf{A}^{S}\right) .
$$

The proposition follows from the above factorization and Prop. 5.4 .

5.5. A $p$-adic measure of Siegel-Eisenstein series. Let $\mathcal{K}_{\mathfrak{c} p^{n}}$ be the ray-class field of $\mathcal{K}$ of conductor $\mathfrak{c} p^{n}(n=1,2, \ldots)$ and let $\mathcal{K}_{\mathfrak{c} p^{\infty}}=\cup_{n} \mathcal{K}_{\mathfrak{c} p^{n}}$. Let $\mathfrak{G}_{\mathfrak{c}}=$

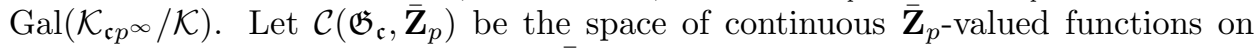
$\mathfrak{G}_{\mathfrak{c}}$. Henceforth, functions in $\mathcal{C}\left(\mathfrak{G}_{\mathfrak{c}}, \overline{\mathbf{Z}}_{p}\right)$ will be implicitly regarded as continuous functions on $\mathbf{A}_{\mathcal{K}}^{\times} / \mathcal{K}^{\times}$by the geometrically normalized reciprocity law as in 1.3 Define a subset $\mathfrak{X}_{\mathfrak{G}_{\mathfrak{c}}}^{+}$of locally algebraic $p$-adic Galois characters by

$$
\mathfrak{X}_{\mathfrak{G}_{\mathfrak{c}}}^{+}=\left\{\widehat{\chi} \in \operatorname{Hom}_{\text {cont }}\left(\mathfrak{G}_{\mathfrak{c}}, \overline{\mathbf{Z}}_{p}^{\times}\right) \mid \chi \text { has infinity type of } k \Sigma, k>4\right\} .
$$

Note that $\mathfrak{X}_{\mathfrak{G}_{\mathfrak{c}}}^{+}$is Zariski dense in $\mathcal{C}\left(\mathfrak{G}_{\mathfrak{c}}, \overline{\mathbf{Z}}_{p}\right)$.

Theorem 5.8. There exists a unique $p$-adic measure $\mathcal{E}_{2,2}$ with value in $V_{p}(\mathbf{G}, \mathbf{K})$ such that for $\hat{\chi} \in \mathfrak{X}_{\mathfrak{G}_{\mathfrak{c}}}^{+}$, we have

$$
\int_{\mathfrak{G}_{\mathfrak{c}}} \widehat{\chi} d \mathcal{E}_{2,2}=\widehat{E}(\chi, \mathfrak{n}),
$$

where $\widehat{E}(\chi, \mathfrak{n})$ is the p-adic avatar of $E(\chi, \mathfrak{n})$.

Proof. Let $\hat{\chi} \in \mathfrak{X}_{\mathfrak{G}_{\mathfrak{c}}}^{+}$and let $\chi$ be the corresponding Hecke character of infinity type $k \Sigma$. Let $g_{1}=\mathbf{m}\left(A_{1}, 1\right) \boldsymbol{\xi}^{-1} \in \mathbf{G}\left(\mathbf{A}_{f}^{S \cup S_{p}}\right)$ and let $\left(a_{1}, a_{2}\right) \in \operatorname{Aut}_{\mathcal{O}_{\mathcal{F}}}\left(\mathbf{L}_{\Sigma}^{0}\right) \times$ $\operatorname{Aut}_{\mathcal{O}_{\mathcal{F}}}\left(\mathbf{L}_{\Sigma^{c}}^{0}\right)=\mathrm{GL}_{2}\left(\mathcal{O} \otimes \mathbf{z} \mathbf{Z}_{p}\right)$. At the $p$-adic cusp $\left(\left[g_{1}\right],\left(a_{1}, a_{2}\right)\right)$, the $p$-adic Eisenstein series $\widehat{E}(\chi, \mathfrak{n})$ has the Fourier expansion

$$
\sum_{\substack{\beta \in \mathcal{H}_{2}(\mathcal{F}), \sigma(\beta)>0 \text { for all } \sigma \in \Sigma}} \mathbf{a}_{\left[g_{1}\right]}^{\left(a_{1}, a_{2}\right)}(\beta, \widehat{E}(\chi, \mathfrak{n})) q^{\beta} .
$$

Set $A:=A_{1} \times\left(a_{1}, a_{2}\right) \in \mathrm{GL}_{2}\left(\mathbf{A}_{f}\right)$ and

$$
\mathcal{I}_{\beta}(A, \widehat{\chi}):=\iota_{p}\left[\mathbf{a}_{A^{*} \beta A}(\chi, \mathfrak{n}) \operatorname{det} \beta^{(k-2) \Sigma}\right] \operatorname{det}\left(a_{1} a_{2}\right)^{k \Sigma_{p}} .
$$

Then we have

$$
\begin{aligned}
& \mathbf{a}_{\left[g_{1}\right]}^{\left(a_{1}, a_{2}\right)}(\beta, \widehat{E}(\chi, \mathfrak{n})) \\
& \quad=\operatorname{det} a_{2}^{k \Sigma_{p}} \cdot \iota_{p}\left(\mathbf{a}_{\beta}\left(\mathbf{m}(A, 1) \boldsymbol{\xi}^{-1}, E(\chi, \mathfrak{n})\right)\right) \\
& \quad=\operatorname{det} a_{2}^{k \Sigma_{p}} \cdot \iota_{p}\left[\chi_{\Sigma_{p}}^{-1}\left(\operatorname{det} a_{1}\right) \chi_{\Sigma_{p}^{c}}^{-1}\left(\operatorname{det} a_{2}\right)\left|\operatorname{det} a_{2} a_{1}\right|_{p}^{2} \mathbf{a}_{A^{*} \beta A}(\chi, \mathfrak{n})(\operatorname{det} \beta)^{(k-2) \Sigma}\right] \\
& \quad=\mathcal{I}_{\beta}(A, \widehat{\chi}) \cdot \widehat{\chi}_{\Sigma_{p}}^{-1}\left(\operatorname{det} a_{1}\right) \widehat{\chi}_{\Sigma_{p}^{c}}^{-1}\left(\operatorname{det} a_{2}\right) .
\end{aligned}
$$

By (5.11),

$$
\begin{aligned}
\mathcal{I}_{\beta}(A, \widehat{\chi})= & \widehat{\chi}\left(\operatorname{det} A^{-1} \operatorname{det} \beta\right) \cdot \prod_{\substack{v \notin S \cup S_{p}, v\left(\operatorname{det} A^{*} \beta A\right)>0}} R_{\beta, v}\left(\widehat{\chi}_{+, v}\left(\varpi_{v}\right)\right) \cdot \prod_{v \in S} \widehat{\chi}_{v}\left(\operatorname{det} \mathbf{u}^{-1}\right) \\
& \times|\operatorname{det} \beta|_{p}^{-2}(\operatorname{det} \beta)^{-2 \Sigma} \cdot \mathbb{I}_{\Xi}\left(A^{*} \beta A\right) \cdot\left|\operatorname{det} A_{1}\right|_{\mathbf{A}_{\mathcal{K}}^{(p)}}^{2} \cdot c_{S}(\mathcal{K}, \mathcal{L}),
\end{aligned}
$$


so we find by inspection that there exist some $b_{i} \in \mathcal{O} \subset \overline{\mathbf{Z}}_{(p)}$ and $c_{i} \in \mathbf{A}_{\mathcal{K}, f}^{\times}$only depending on $A$ and $\beta$ such that

$$
\mathbf{a}_{\left[g_{1}\right]}^{\left(a_{1}, a_{2}\right)}(\beta, \widehat{E}(\chi, \mathfrak{n}))=\sum_{i} b_{i} \cdot \widehat{\chi}\left(c_{i}\right) .
$$

Therefore, it follows from the Zariski density of $\mathfrak{X}_{\mathfrak{G}_{\mathfrak{c}}}^{+}$in $\mathcal{C}\left(\mathfrak{G}_{\mathfrak{c}}, \overline{\mathbf{Z}}_{p}\right)$ and the abstract Kummer congruences (Kat78, (4.0.6)]) that (5.12) gives rise to a $p$-adic measure $\mathcal{E}_{2,2}$ on $\mathfrak{G}_{\mathfrak{c}}$ with value in the space of formal $q$-expansions. Moreover, by the $q$ expansion principle for $p$-adic modular forms on unitary Shimura varieties, we conclude that $\mathcal{E}_{2,2}$ descends to the desired $V_{p}(\mathbf{G}, \mathbf{K})$-valued Eisenstein measure.

\subsection{Pull-backs of Siegel Eisenstein series.}

5.6.1. Let $G=G U(V)$ and let $P$ be the stabilizer of the flag $\{0\} \subset \mathcal{K} x^{1} \subset V$ in $G$. The group $G_{P}:=G U(W)$ of unitary $\mathcal{F}$-similitudes is the algebraic group $\underline{\mathcal{K}}^{\times}$over $\mathcal{F}$ such that $\underline{\mathcal{K}}^{\times}(\mathcal{F})=\mathcal{K}^{\times}$and the unitary $U(W)$ is the norm-one subgroup $\underline{\mathcal{K}}^{1}$ of $\underline{\mathcal{K}}^{\times}$. The unipotent radical $N_{P}$ and the standard Levi subgroup $M_{P}$ of $P$ are given by

$$
N_{P}=\left\{n(w, x):=\left[\begin{array}{ccc}
1 & w^{*} \vartheta^{-1} & x+\frac{1}{2} w \vartheta^{-1} w^{*} \\
& 1 & w \\
& & 1
\end{array}\right] \mid w \in \mathcal{K}, x \in \mathcal{F}\right\}
$$

and

$$
M_{P}=\left\{m(a, h):=\left[\begin{array}{lll}
\nu(h) a^{-*} & & \\
& h & \\
& & a
\end{array}\right] \mid a \in \mathcal{K}^{\times}, h \in G_{P}\right\} .
$$

The Hermitian symmetric domain associated to $G$ is $X_{2,1}$. Put

$$
K_{\infty}^{0}=\left\{g \in G\left(\mathcal{F} \otimes_{\mathbf{Q}} \mathbf{R}\right) \mid g \mathbf{i}_{0}=\mathbf{i}_{0}\right\} \quad\left(\mathbf{i}_{0}:=\left(\left[\begin{array}{c}
\sqrt{-1} \\
0
\end{array}\right]\right)_{\sigma \in \Sigma} \in X_{2,1}\right) .
$$

Recall that $K_{v}^{0}$ is the stabilizer of $M \otimes_{\mathcal{O}_{\mathcal{K}}} \mathcal{O}_{\mathcal{K}_{v}}$ if $v \in \mathbf{h}$.

We introduce some special open-compact subgroups. For an integral ideal $\mathfrak{a}$ of $\mathcal{O}_{\mathcal{K}}$, put

$$
\mathcal{U}(\mathfrak{a})=\prod_{v \in \mathbf{h}} \mathcal{U}(\mathfrak{a})_{v}, \text { where } \mathcal{U}(\mathfrak{a})_{v}:=\left(1+\mathfrak{a}_{v}\right)^{\times}
$$

and define a subgroup $D_{W}(\mathfrak{a})$ of $U(W)\left(\mathbf{A}_{f}\right)$ by

$$
D_{W}(\mathfrak{a})=\mathcal{U}(\mathfrak{a}) \cap U(W)\left(\mathbf{A}_{f}\right)=\mathcal{U}(\mathfrak{a}) \cap \mathcal{K}_{\mathbf{A}_{f}}^{1} .
$$

Set

$$
K_{P}(\mathfrak{n}):=D_{W}(\mathfrak{d} \mathfrak{n})
$$

Let $X_{W}(\mathfrak{n})$ be a set of representatives of the finite set $U(W)(\mathcal{F}) \backslash U(W)\left(\mathbf{A}_{f}\right) / K_{P}(\mathfrak{n})$ in $U(W)\left(\mathbf{A}_{f}^{(p)}\right)$. In addition to (a1), we further assume the auxiliary ideal $\mathfrak{n}$ is sufficiently small such that

$$
h K_{P}(\mathfrak{n}) h^{-1} \cap U(W)(\mathcal{F})=\{1\} \text { for all } h \in X_{W}(\mathfrak{n}) .
$$


For $v \in S$, define an open-compact monoid $D_{V}^{1}\left(\mathfrak{n}_{v}\right)$ of $U(V)\left(\mathcal{F}_{v}\right)$ by

$$
D_{V}^{1}\left(\mathfrak{n}_{v}\right)=\left\{g \in K_{v}^{0} \cap U(V)\left(\mathcal{F}_{v}\right) \mid g \in\left[\begin{array}{ccc}
1+\mathfrak{n}_{v} & \mathfrak{r}_{v} & \mathfrak{r}_{v} \\
\mathfrak{d}_{v} \mathfrak{n}_{v} & \mathfrak{r}_{v} & \mathfrak{d}_{v} \mathfrak{r}_{v} \\
\mathfrak{n}_{v} & \mathfrak{n}_{v} & 1+\mathfrak{n}_{v}
\end{array}\right]\right\} .
$$

It will be verified in Lemma 6.2 that $D_{V}^{1}\left(\mathfrak{n}_{v}\right)$ is indeed a subgroup of $U(V)\left(\mathcal{F}_{v}\right)$. Let $D_{V}\left(\mathfrak{n}_{v}\right)$ be the open-compact subgroup of $G\left(\mathcal{F}_{v}\right)$ given by

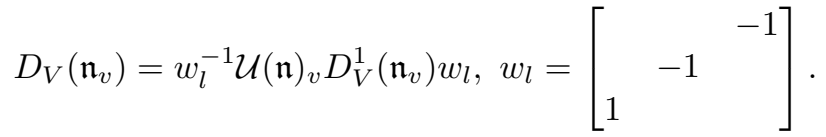

Here $\mathcal{U}(\mathfrak{n})_{v}=\left(1+\mathfrak{n}_{v}\right)^{\times}$is regarded as a subgroup of the center of $G\left(\mathcal{F}_{v}\right)$. Define an open-compact subgroup $K$ in $G\left(\mathbf{A}_{f}\right)$ by

$$
K=\prod_{v \notin S} K_{v}^{0} \times \prod_{v \in S} D_{V}\left(\mathfrak{n}_{v}\right) .
$$

5.6.2. Pull-back formula. Let $\chi$ be the Hecke character of $\mathcal{K}^{\times}$as in the previous section. Assume that $\chi$ has infinity type $k \Sigma, k>4$. For each place $v$ of $\mathcal{F}$ and $s \in \mathbf{C}$, let $I_{v}(\chi, \mathbf{1}, s)$ denote the space of smooth and $K_{v}^{0}$-finite functions $f: G\left(\mathcal{F}_{v}\right) \rightarrow \mathbf{C}$ such that

$$
f(m(a, h) n g)=\chi_{v}^{-1}(a)\left|\nu(h)^{-1} \bar{a} a\right|^{-s} f(g) \quad\left(n \in N_{P}\left(\mathcal{F}_{v}\right)\right)
$$

and let $I(\chi, \mathbf{1}, s)=\otimes_{v}^{\prime} I_{v}(\chi, \mathbf{1}, s)$ be the restricted tensor product with respect to the spherical sections $f_{\chi, \mathbf{1}, s, v}^{0} \in I_{v}(\chi, \mathbf{1}, s)$ with $f_{\chi, \mathbf{1}, s, v}^{0}\left(K_{v}^{0}\right)=1$. Consider the diagonal embedding

$$
i: U(V) \times U(W) \hookrightarrow U(\mathbf{W}), \quad i(g, h):=(g, h) \in \mathrm{GL}(V \oplus W)=\mathrm{GL}(\mathbf{W}) .
$$

As an embedding between matrix groups, $i$ can be written as

$$
i(g, h)=\Delta^{-1}\left[\begin{array}{ll}
g & \\
& h
\end{array}\right] \Delta, \Delta:=\left[\begin{array}{cccc}
1 & & & \\
& 1 & & \frac{\vartheta}{2} \\
& & 1 & \\
& -1 & & \frac{\vartheta}{2}
\end{array}\right] \in \mathrm{GL}_{4}(\mathcal{K}) .
$$

For each place $v$ of $\mathcal{F}$ and $\phi_{v} \in I_{v}(\chi, s)$, define the local pull-back section $\phi_{v}^{p b} \in$ $I_{v}(\chi, \mathbf{1}, s)$ by

$$
\phi_{v}^{p b}\left(g_{v}\right)=\int_{U(W)\left(\mathcal{F}_{v}\right)} \phi\left(i\left(g_{v}, h_{v} h_{v}^{\prime}\right)\right) \chi\left(h_{v} h_{v}^{\prime}\right) d h_{v},
$$

where $h_{v}^{\prime} \in \mathcal{K}_{v}^{\times}$such that $h_{v}^{\prime} \overline{h^{\prime}} v=\nu\left(g_{v}\right)$. For a decomposable global section $\phi=$ $\otimes_{v} \phi_{v} \in I(\chi, s)$, we denote by $\phi^{p b}:=\otimes_{v} \phi_{v}^{p b} \in I(\chi, \mathbf{1}, s)$ the global pull-back section of $\phi$.

Lemma 5.9. Let $\phi=\otimes \phi_{v} \in I(\chi, s)$. Then

(i) The local section $\phi_{v}^{p b}$ at each place $v$ converges absolutely for Res $>-\frac{k}{2}$.

(ii) The global section $\phi^{p b}$ converges absolutely for Re $s>1-\frac{k}{2}$.

In particular, $\phi^{p b}$ converges absolutely at $s=0$. 
Proof. To prove part (i), it suffices to show that $\phi_{v}^{p b}(1)$ converges absolutely for every $\phi_{v} \in I_{v}(\chi, s)$. If $v$ is non-split, this is clear. Suppose that $v=w \bar{w}$ is split. With the identifications $U(W)\left(\mathcal{F}_{v}\right)=\mathcal{F}_{v}^{\times}$and $U(\mathbf{W})\left(\mathcal{F}_{v}\right)=\mathrm{GL}_{4}\left(\mathcal{F}_{v}\right)$ in (1.2), we can write

$$
\phi_{v}^{p b}(1)=\int_{\mathcal{F}_{v}^{\times}} \phi_{v}(i(1, x)) \chi_{w} \chi_{w}^{-1}(x) d^{\times} x .
$$

The argument of the proof in [Yan97, Prop. 1.7] shows that there exist complex functions $a_{1}(s), a_{2}(s)$ and a sufficiently large positive integer $n$ such that

$$
\begin{aligned}
& \phi_{v}(i(1, x))=a_{1}(s) \cdot \chi_{w}^{-1}(x)|x|^{-s} \text { if }|x|>p^{n}, \\
& \phi_{v}(i(1, x))=a_{2}(s) \cdot \chi_{\bar{w}}(x)|x|^{s} \text { if }|x|<p^{-n} .
\end{aligned}
$$

Therefore,

$$
\begin{aligned}
\phi_{v}^{p b}(1)= & \int_{p^{-n} \leq|x| \leq p^{n}} \phi_{v}(i(1, x)) \chi_{w} \chi_{\bar{w}}^{-1}(x) d^{\times} x \\
& +a_{1}(s) \cdot \int_{|x|<p^{-n}} \chi_{\bar{w}}(x)|x|^{s} d^{\times} x+a_{2}(s) \int_{|x|<p^{-n}} \chi_{w}(x)|x|^{s} d^{\times} x .
\end{aligned}
$$

The last two integrals converge absolutely for $\operatorname{Re} s>-\frac{k}{2}$ since $\left|\chi_{w}\left(\varpi_{v}\right)\right|=$ $\left|\chi_{\bar{w}}\left(\varpi_{v}\right)\right|=\left|\varpi_{v}\right|_{\mathcal{F}_{v}}^{\frac{k}{2}}$ (note that the first two $|\cdot|$ mean the usual absolute value on complex numbers), from which the split case follows.

Part (ii) is a consequence of part (i), Prop. $5.11(2)$ and the absolute convergence of the Euler products of $L$-functions $L(s, \chi)$ and $L\left(2 s, \chi_{+}\right)$for $\operatorname{Re} s>1-\frac{k}{2}$.

The adelic Eisenstein series $E_{\mathbf{A}}(g, f)$ associated to $f \in I(\chi, \mathbf{1}, s)$ is defined by

$$
E_{\mathbf{A}}(g, f)=\sum_{\gamma \in P(\mathcal{F}) \backslash G(\mathcal{F})} f(\gamma g) \quad(g \in G(\mathbf{A})) .
$$

The series $E_{\mathbf{A}}(g, f)$ converges absolutely for Re $s$ large enough by the general theory of Eisenstein series Lan76]. Moreover, if $f$ is a pull-back section $\phi^{p b}$ for some $\phi \in I(\chi, s)$, then we have the following pull-back formula.

Theorem 5.10 (The pull-back formula).

$$
E_{\mathbf{A}}\left(g, \phi^{p b}\right)=\int_{U(W)(\mathcal{F}) \backslash U(W)(\mathbf{A})} E_{\mathbf{A}}\left(i\left(g, h h^{\prime}\right), \phi\right) \chi\left(h h^{\prime}\right) d h,
$$

where $h^{\prime} \in \mathbf{A}_{\mathcal{K}}^{\times}$such that $h \overline{h^{\prime}}=\nu(g)$.

Proof. Since $U(W)$ is a totally definite unitary group, by [Shi97, Prop. 2.4] the embedding $(g, h) \rightarrow i(g, h)$ gives rises to a bijection $P(\mathcal{F}) \backslash G(\mathcal{F}) \times U(W)(\mathcal{F}) \stackrel{\sim}{\rightarrow}$ $Q(\mathcal{F}) \backslash \mathbf{G}(\mathcal{F})$. The theorem follows.

5.6.3. The pull-back sections. Let $\phi_{\chi, s}=\otimes_{v} \phi_{\chi, s, v}$ be the section in $I(\chi, s)$ defined in Def. 5.6. Put

$$
\phi_{\chi, s}^{\Upsilon}(g)=\phi_{\chi, s}(g \Upsilon), \Upsilon=\left[\begin{array}{cccc}
1 & & & \\
& \frac{1}{2} & & -\frac{1}{2} \\
& \vartheta^{-1} & & \vartheta^{-1}
\end{array}\right]=\Delta^{-1} \in \mathrm{GL}_{4}\left(O_{p}\right) \hookrightarrow U(\mathbf{W})\left(\mathcal{F}_{p}\right) .
$$

We will need to calculate the pull-back section $\left(\phi_{\chi, s}^{\Upsilon}\right)^{p b}=\otimes_{v \nmid p} \phi_{\chi, s, v}^{p b} \otimes_{v \mid p}\left(\phi_{\chi, s, v}^{\Upsilon}\right)^{p b}$. First we compute the local pull-back section $\left.\phi_{\chi, s, v}^{p b}\right|_{s=0}$ at archimedean or unramified 
places $v$ and postpone the calculations of $\phi_{\chi, s, v}^{p b}$ for $v \in S$ and $\left(\phi_{\chi, s, v}^{\Upsilon}\right)^{p b}$ for $v \mid p$ to the next section. Let

$$
J_{k}\left(g_{\infty}, \tau\right):=\rho^{k I_{-}}\left(J\left(g_{\infty}, \tau\right)\right),\left(g_{\infty}, \tau\right) \in G\left(\mathcal{F} \otimes_{\mathbf{Q}} \mathbf{R}\right) \times X_{2,1}
$$

be the automorphy factor of weight $k I_{-}=(0,0 ; k \Sigma)$ defined in $\$ 3.4$

Proposition 5.11. Let $f_{k, \infty}\left(g_{\infty}\right)=J_{k}\left(g_{\infty}, \mathbf{i}_{0}\right)^{-1} \in I_{\infty}(\chi, 0)$.

(1) $\left.\left(\phi_{\chi, s, \infty}\right)^{p b}\right|_{s=0}=f_{k, \infty}$.

(2) If $v \notin S \cup S_{p}$ is a finite place, then

$$
\phi_{\chi, s, v}^{p b}=\left(f_{\chi, s, v}^{0}\right)^{p b}=\frac{L\left(s, \chi_{v}\right)}{L\left(2 s, \chi_{+, v}\right)} f_{\chi, \mathbf{1}, s, v}^{0} .
$$

Proof. Part (1) is straightforward. Part (2) follows from a classical integral in the doubling method for $U(1) \times U(1) \hookrightarrow U(1,1)$ (GPSR87] and [Li92]).

We remark that by using Lemma 5.3 and Prop. 6.3 combined with the definitions of $\phi_{\chi, s, v}$, it is not difficult to show that $\left(\phi_{\chi, s}^{\Upsilon}\right)^{p b}$ is invariant by the open-compact subgroup $K_{1}^{n}$ for some large $n$ ( $K$ is as (15.15) ).

Lemma 5.12. The function $(g, h) \mapsto \phi_{\chi, s}^{\Upsilon}(i(g, h)) \chi(h)$ on $U(V)\left(\mathbf{A}_{f}\right) \times U(W)\left(\mathbf{A}_{f}\right)$ is invariant by the right translation of $K_{1}^{n} \times K_{P}(\mathfrak{n})$ for some large $n$.

Proof. The only non-trivial point is to show the right invariance by $I_{1}\left(p^{n}\right)_{v} \times$ $K_{P}(\mathfrak{n})_{v}$ for $v \mid p$. Recall that with the identifications in (1.2): $\operatorname{GL}_{4}\left(\mathcal{F}_{v}\right)=U(\mathbf{W})\left(\mathcal{F}_{v}\right)$ and

$$
\mathcal{F}_{v}^{\times}=U(W)\left(\mathcal{F}_{v}\right)=\left\{\left(h, h^{-1}\right) \mid h \in \mathcal{F}_{v}^{\times}\right\}, \quad h \rightarrow\left(h, h^{-1}\right) .
$$

Then $\mathcal{O}_{\mathcal{F}_{v}}^{\times}=\mathcal{O}_{\mathcal{K}_{v}}^{\times} \cap \mathcal{K}_{v}^{1}=K_{P}(\mathfrak{n})_{v}$. By (5.16), we have $i(u, t) \Upsilon=\Upsilon\left[\begin{array}{ll}u & 0 \\ 0 & t\end{array}\right]$, and for $u \in I_{1}\left(p^{n}\right)_{v}$ and $t \in \mathcal{O}_{\mathcal{F}_{v}}^{\times}$,

$$
\phi_{\chi, s}^{\Upsilon}(i(g u, h t)) \chi\left(h\left(t, t^{-1}\right)\right)=\phi_{\chi, s}\left(i(g, h) \Upsilon\left[\begin{array}{ll}
u & 0 \\
0 & t
\end{array}\right]\right) \chi(h) \chi_{w} \chi_{\bar{w}}^{-1}(t) \quad\left(w \in \Sigma_{p}, w \mid v\right) .
$$

By Lemma 5.3, for $n$ large the last term in the above equation equals

$$
\phi_{\chi, s}(i(g, h) \Upsilon) \chi(h) \chi_{w} \chi_{\bar{w}}^{-1}\left(u^{-1}\right) \chi_{w} \chi_{\bar{w}}^{-1}(u)=\phi_{\chi, s}(i(g, h) \Upsilon) \chi(h)=\phi_{\chi, s}^{\Upsilon}(i(g, h)) \chi(h) .
$$

This completes the proof.

5.6.4. Normalized Eisenstein series. Define the normalized adelic Eisenstein series $E_{\mathbf{A}}(g, \chi \mid \mathbf{1}, \mathfrak{n})$ associated to the pull-back section $\left(\phi_{\chi, s}^{\Upsilon}\right)^{p b}$ by

$$
\begin{aligned}
E_{\mathbf{A}}(g, \chi \mid \mathbf{1}, \mathfrak{n}) & =\frac{1}{\operatorname{vol}\left(K_{P}(\mathfrak{n})\right)} \cdot E_{\mathbf{A}}\left(g \Upsilon,\left(\phi_{\chi, s}^{*}\right)^{p b}\right) \quad(g \in G(\mathbf{A})) \\
& =\frac{\Lambda_{2}^{S \cup S_{p}}(s, \chi)}{\operatorname{vol}\left(K_{P}(\mathfrak{n})\right)} \cdot E_{\mathbf{A}}\left(g,\left(\phi_{\chi, s}^{\Upsilon}\right)^{p b}\right) .
\end{aligned}
$$

The series $E_{\mathbf{A}}(g, \chi \mid \mathbf{1}, \mathfrak{n})$ converges absolutely for $\operatorname{Re} s>2-\frac{k}{2}$. We define the C-valued function $E(\chi \mid \mathbf{1}, \mathfrak{n})$ on $X_{2,1} \times G\left(\mathbf{A}_{f}\right)$ by

$$
\begin{aligned}
E(\chi \mid \mathbf{1}, \mathfrak{n})(\tau, g)= & \left.\chi(\nu(g)) J_{k}\left(g_{\infty}, \mathbf{i}_{0}\right) E_{\mathbf{A}}\left(\left(g_{\infty}, g\right), \chi \mid \mathbf{1}, \mathfrak{n}\right)\right|_{s=0} \\
& \times\left(g_{\infty} \in G\left(\mathcal{F} \otimes_{\mathbf{Q}} \mathbf{R}\right)^{+}, g_{\infty} \mathbf{i}_{0}=\tau\right) .
\end{aligned}
$$


Then $E(\chi \mid \mathbf{1}, \mathfrak{n})$ is a holomorphic Eisenstein series on $G$ of weight $k I^{-}=(0,0 ; k \Sigma)$. By the pull-back formula Theorem 5.10 and Lemma 5.12, we have

$$
\begin{aligned}
E(\chi \mid \mathbf{1}, \mathfrak{n})(\tau, g) & =\frac{1}{\operatorname{vol}\left(K_{P}(\mathfrak{n})\right)} \cdot \int_{U(W)(\mathcal{F}) \backslash U(W)\left(\mathbf{A}_{f}\right)} E(\chi, \mathfrak{n})\left(Z_{\tau}, i(g, h) \Upsilon\right) \cdot \chi(h) d h \\
& =\sum_{h \in X_{W}(\mathfrak{n})} E(\chi, \mathfrak{n})\left(Z_{\tau}, i(g, h) \Upsilon\right) \cdot \chi(h) \quad\left(g \in U(V)\left(\mathbf{A}_{f}\right)\right) .
\end{aligned}
$$

Regarding $E(\chi, \mathfrak{n})$ and $E(\chi \mid \mathbf{1}, \mathfrak{n})$ as geometric modular forms over $\mathbf{C}$, by the discussion in $₫ 2.6$, we find that

$E(\chi, \mathfrak{n})\left(i_{V, W}\left([\tau, g],\left[\mathbf{x}_{0}, h\right]\right),\left(2 \pi i d \underline{z}_{V}, 2 \pi i d z_{W}^{(1)}\right)\right)=E(\chi, \mathfrak{n})\left(\left[Z_{\tau}, i(g, h) \Upsilon\right], 2 \pi i d \underline{z}_{\mathbf{W}}\right)$. Combining (5.20), we thus obtain

$$
\begin{aligned}
E(\chi \mid \mathbf{1}, \mathfrak{n})\left([\tau, g], 2 \pi i d \underline{z}_{V}\right)= & \sum_{h \in X_{W}(\mathfrak{n})} E(\chi, \mathfrak{n})\left(i_{V, W}\left([\tau, g],\left[\mathbf{x}_{0}, h\right]\right),\right. \\
& \left.\times\left(2 \pi i d \underline{z}_{V}, 2 \pi i d \underline{z}_{W}\right)\right) \chi(h) .
\end{aligned}
$$

5.6.5. The p-adic Eisenstein series. Let $\widehat{E}(\chi, \mathfrak{n}) \in V_{p}(\mathbf{G}, \mathbf{K})$ be the $p$-adic avatar of the Eisenstein series $E(\chi, \mathfrak{n})$. Recall that $\mathcal{W}_{p}$ is the $p$-adic completion of the ring of integers of the maximal unramified extension of $\mathbf{Q}_{p}$ in $\mathbf{C}_{p}$. We define the $p$-adic Eisenstein series $\widehat{E}(\chi \mid \mathbf{1}, \mathfrak{n})$ over $\mathcal{W}_{p}$ by the formulas

$$
\widehat{E}(\chi \mid \mathbf{1}, \mathfrak{n})(\underline{A})=\sum_{h \in X_{W}(\mathfrak{n})} \widehat{E}(\chi, \mathfrak{n})\left(\underline{A} \times \underline{\mathcal{B}}_{u h}\right) \cdot \widehat{\chi}(u h),
$$

where $\underline{A} \in I_{U(V)}^{0}\left(K_{1}^{n}, \mathbf{c}\right)$ with $\mathbf{c}=u \bar{u} \in \mathrm{Cl}_{\mathcal{F}}^{+}(K)$ is a test quintuple and $\underline{\mathcal{B}}$ is the universal family over $I_{U(W)}^{0}\left(K_{P}(\mathfrak{n})^{n}, \mathbf{c}\right) / \mathcal{W}_{p}$.

Definition 5.13. Let $e$ be the Hida idempotent for $G=G U(V)$ as in $\$ 4.3$. Define the ordinary $p$-adic Eisenstein series $\widehat{E}^{\text {ord }}(\chi \mid \mathbf{1}, \mathfrak{n})$ on $G$ by

$$
\widehat{E}^{\text {ord }}(\chi \mid \mathbf{1}, \mathfrak{n})=e . \widehat{E}(\chi \mid \mathbf{1}, \mathfrak{n}) .
$$

Let $\boldsymbol{\omega}_{\mathcal{B}}, d^{\times} t$ and $d \underline{z}_{W}$ be the differential forms on $\underline{\mathcal{B}}$ introduced in $\$ 2.8$. For each $\mathcal{O}_{\mathcal{K}}$-basis $\boldsymbol{\omega}$ of $\Omega_{A}$, by the relation (2.23) we find that

$$
\begin{aligned}
E(\chi, \mathfrak{n})\left(\underline{A} \times \underline{\mathcal{B}}_{u h},\left(\boldsymbol{\omega}, \boldsymbol{\omega}_{\mathcal{B}}\right)\right) & =\frac{1}{\Omega_{p}^{k \Sigma}} \cdot E(\chi, \mathfrak{n})\left(\underline{A} \times \underline{\mathcal{B}}_{u h},\left(\boldsymbol{\omega}, d^{\times} t\right)\right) \\
& =\frac{(2 \pi i)^{k[\mathcal{F}: \mathbf{Q}]}}{\Omega_{\infty}^{k \Sigma}} \cdot E(\chi, \mathfrak{n})\left(\underline{A}_{\infty} \underline{\mathcal{B}}_{u h},\left(\boldsymbol{\omega}, 2 \pi i d \underline{z}_{W}\right)\right),
\end{aligned}
$$

where

$$
\Omega_{\bullet}^{k \Sigma}=\prod_{\sigma \in \Sigma} \Omega_{\bullet, \sigma}^{k}, \quad \bullet \in\{\infty, p\}
$$

Put

$$
E^{\text {ord }}(\chi \mid \mathbf{1}, \mathfrak{n})=\frac{(2 \pi i)^{k[\mathcal{F}: \mathbf{Q}]}}{\Omega_{\infty}^{k \Sigma}} \cdot e \cdot E(\chi \mid \mathbf{1}, \mathfrak{n})
$$

Combining (5.21) and (5.22), we find that

$$
\frac{1}{\Omega_{p}^{k \Sigma}} \cdot \widehat{E}^{\text {ord }}(\chi \mid \mathbf{1}, \mathfrak{n}) \text { is the } p \text {-adic avatar of } E^{\text {ord }}(\chi \mid \mathbf{1}, \mathfrak{n}) \text {. }
$$


Proposition 5.14. There exists a unique p-adic measure $\mathcal{E}_{2,1}^{\text {ord }}$ with values in $V_{p}(G, K) \otimes \mathbf{z}_{p} \mathcal{W}_{p}$ such that for every $\widehat{\chi} \in \mathfrak{X}_{\mathfrak{G}_{\mathfrak{c}}}^{+}$,

$$
\int_{\mathfrak{G}_{\mathfrak{c}}} \widehat{\chi} d \varepsilon_{2,1}^{\text {ord }}=\widehat{E}^{\text {ord }}(\chi \mid \mathbf{1}, \mathfrak{n})
$$

Proof. For $\varphi \in \mathcal{C}\left(\mathfrak{G}_{\mathfrak{c}}, \mathcal{W}_{p}\right)$ and $h \in U(W)\left(\mathbf{A}_{f}\right)$, we put $\varphi \mid h(x)=\varphi(x h)$. We define a $p$-adic measure $\mathcal{E}_{2,1}^{o r d}$ with values in $V_{p}(G, K) \otimes_{\mathbf{z}_{p}} \mathcal{W}_{p}$ by the rule:

$$
\int_{\mathfrak{G}_{\mathfrak{c}}} \varphi d \mathcal{E}_{2,1}^{\text {ord }}(\underline{A})=\sum_{h \in X_{W}(\mathfrak{n})} e \cdot \int_{\mathfrak{G}_{\mathfrak{c}}} \varphi \mid h d \mathcal{E}_{2,2}\left(\underline{A} \times \underline{\mathcal{B}}_{h}\right) .
$$

The evaluation property immediately follows from the constructions of $\mathcal{E}_{2,2}$ and $\widehat{E}^{\text {ord }}(\chi \mid \mathbf{1}, \mathfrak{n})$.

5.7. The $\Lambda$-adic Eisenstein series. Let $\Delta_{\mathfrak{c}}$ be the torsion subgroup of $\mathfrak{G}_{\mathfrak{c}}=$ $\operatorname{Gal}\left(\mathcal{K}_{\mathfrak{c} p \infty} / \mathcal{K}\right)$. Let $\psi: \Delta_{\mathfrak{c}} \rightarrow \overline{\mathbf{Q}}^{\times} \hookrightarrow \mathbf{C}_{p}^{\times}$be a branch character and let $\Delta:=\psi\left(\Delta_{\mathfrak{c}}\right)$. We will have to work over a large base ring $\mathcal{O}_{\mathfrak{p}}$ defined as follows. Let $N_{0}=\mathbf{N}_{\mathcal{F} / \mathbf{Q}}(\mathfrak{n})$ and let $\mathcal{K}:=\mathcal{K}^{n c}\left(\Delta, e^{2 \pi i / N_{0}}\right)$ be a finite extension of $\mathcal{K}^{n c}$. Thus $\mathcal{K}$ is unramified at $p$ if $\sharp(\Delta)$ is prime to $p$. Let $\mathfrak{p}$ be the prime ideal of $\mathcal{K}$ induced by $\iota_{p}: \overline{\mathbf{Q}} \hookrightarrow \mathbf{C}_{p}$ and let $\mathcal{O}=\mathcal{O}_{\mathcal{K},(\mathfrak{p})}$ be the localization at $\mathfrak{p}$. Henceforth, we fix $\mathcal{O}_{\mathfrak{p}} \supset \mathcal{W}_{p}$ to be the $p$-adic closure of the ring generated by $\mathcal{O}$ over the ring $\mathcal{W}_{p}$ of integers of the maximal unramified extension of $\mathbf{Q}_{p}$ in $\mathbf{C}_{p}$.

Let $\mathcal{K}_{\infty}$ be the compositum of the cyclotomic $\mathbf{Z}_{p}$-extension and the anticyclotomic $\mathbf{Z}_{p}^{d}$-extension of $\mathcal{K}$. Let $\Gamma_{\mathcal{K}}=\operatorname{Gal}\left(\mathcal{K}_{\infty} / \mathcal{K}\right)$ and let $\Lambda=\mathcal{O}_{\mathfrak{p}} \llbracket \operatorname{Gal}\left(\mathcal{K}_{\infty} / \mathcal{K}\right) \rrbracket$ be a $(d+1)$-variable Iwasawa algebra over $\mathcal{O}_{\mathfrak{p}}$. Let $\mathcal{K}_{S}$ be the maximal algebraic extension of $\mathcal{K}$ unramified outside places above $S \cup S_{p}$. Denote by $\varepsilon_{\Lambda}: \operatorname{Gal}\left(\mathcal{K}_{S} / \mathcal{K}\right) \rightarrow \Gamma_{\mathcal{K}} \hookrightarrow$ $\Lambda^{\times}$the tautological character and let $\Psi=\psi \varepsilon_{\Lambda}: \operatorname{Gal}\left(\mathcal{K}_{S} / \mathcal{K}\right) \rightarrow \Lambda^{\times}$be the $\Lambda$-valued Galois character attached to $\psi$. Denote by $\Psi_{+}=\Psi \circ V$ the composition of $\Psi$ and the Verschiebung map $V: G_{\mathcal{F}}^{a b} \rightarrow G_{\mathcal{K}}^{a b}$. We shall regard $\Psi$ (resp. $\Psi_{+}$) as a $\Lambda$-valued Hecke character of $\mathcal{K}^{\times}$(resp. $\mathcal{F}^{\times}$) by the geometrically normalized reciprocity law. By definition we have $\Psi_{+}=\left.\Psi\right|_{\mathbf{A} \times}$. Let $\mathfrak{X}_{\Lambda}:=\operatorname{Hom}_{\text {cont }}\left(\Gamma_{\mathcal{K}}, \mathbf{C}_{p}^{\times}\right) \subset \operatorname{Hom}_{\text {cont }}\left(\Lambda, \mathbf{C}_{p}\right)$. Note that for $\widehat{\epsilon} \in \mathfrak{X}_{\Lambda}$, the composition $\widehat{\epsilon} \circ \Psi: \operatorname{Gal}\left(\mathcal{K}_{S} / \mathcal{K}\right) \rightarrow \mathbf{C}_{p}^{\times}$factors through $\mathfrak{G}_{\mathfrak{c}}$. We set

$$
\mathfrak{X}_{\Lambda}^{+}=\left\{\widehat{\epsilon} \in \mathfrak{X}_{\Lambda} \mid \widehat{\epsilon} \circ \Psi \in \mathfrak{X}_{\mathfrak{G}_{\mathfrak{c}}}^{+}\right\},
$$

where $\mathfrak{X}_{\mathfrak{G}_{\mathfrak{c}}}^{+}$is a subset of locally algebraic $p$-adic Galois characters on $\mathfrak{G}_{\mathfrak{c}}$ defined in $\$ 5.5$. By definition, for each $\widehat{\epsilon} \in \mathfrak{X}_{\Lambda}^{+}$, the $p$-adic Galois character $\widehat{\epsilon} \circ \Psi$ is the $p$-adic avatar of a Hecke character $\psi \epsilon$ of infinity type $k \Sigma, k>4$, and the conductor of $\psi \epsilon$ is a factor of $\mathfrak{c} p^{\infty}$.

Let $\pi_{\mathbf{T}}: \mathbf{T} / \mathbf{T}^{g l}=\left(O_{p}^{\times}\right)^{3} / \overline{j\left(\left(\mathcal{O}_{\mathcal{F}, N_{0}}^{\times}\right)^{2}\right)} \rightarrow \Gamma_{\mathcal{K}}$ be the homomorphism defined by

$$
\pi_{\mathbf{T}}\left(t_{1}, t_{2}, t_{3}\right)=\left.\left.\operatorname{rec}_{\Sigma_{p}^{c}}\left(t_{2}\right)\right|_{\mathcal{K}_{\infty}} \operatorname{rec}_{\Sigma_{p}}\left(t_{3}\right)\right|_{\mathcal{K}_{\infty}} .
$$

Denote by $\pi_{\mathbf{T}}^{\psi}: \widetilde{\Lambda}=\mathcal{O}_{\mathfrak{p}} \llbracket \mathbf{T} / \mathbf{T}^{g l} \rrbracket \rightarrow \Lambda$ the associated $\mathcal{O}_{\mathfrak{p}}$-algebra homomorphism given by $\pi_{\mathbf{T}}^{\psi}\left(t_{1}, t_{2}, t_{3}\right)=\Psi_{\Sigma_{p}^{c}}\left(t_{2}\right) \Psi_{\Sigma_{p}}\left(t_{3}\right)\left(\Psi_{\Sigma_{p}}:=\Psi \circ \operatorname{rec}_{\Sigma_{p}}\right)$. Recall that $\mathcal{M}_{\text {ord }}(K, \widetilde{\Lambda})$ is the space of $\widetilde{\Lambda}$-adic ordinary modular forms. We set

$$
\mathcal{M}_{\text {ord }}(K, \Lambda)=\mathcal{M}_{\text {ord }}(K, \widetilde{\Lambda}) \otimes_{\widetilde{\Lambda}, \pi_{\mathrm{T}}^{\psi}} \Lambda
$$


In virtue of Theorem 4.25, we can identify $\mathcal{M}_{\text {ord }}(K, \Lambda)$ with the subspace of $p$-adic measures on $\Gamma_{\mathcal{K}}$ with values in the space of $p$-adic ordinary modular forms such that for $F \in \mathcal{M}_{\text {ord }}(K, \Lambda)$, the eigencharacter of $\mathbf{T}$ under the action [.] defined in Def. 3.4 is given by $\left(\mathbf{1}, \Psi_{\Sigma_{p}^{c}} ; \Psi_{\Sigma_{p}}\right)$ 2

Corollary 5.15. There exists a $\Lambda$-adic form $\mathcal{E}^{\text {ord }}(\Psi \mid \mathbf{1}, \mathfrak{n}) \in \mathcal{M}_{\text {ord }}(K, \Lambda)$ such that at every $\widehat{\epsilon} \in \mathfrak{X}_{\Lambda}^{+}$the specialization $\widehat{\epsilon}\left(\mathcal{E}^{\text {ord }}(\Psi \mid \mathbf{1}, \mathfrak{n})\right)$ is given by

$$
\widehat{\epsilon}\left(\mathcal{E}^{\text {ord }}(\Psi \mid \mathbf{1}, \mathfrak{n})\right)=\widehat{E}^{\text {ord }}(\psi \epsilon \mid \mathbf{1}, \mathfrak{n}) .
$$

Proof. Define the morphism $\mathcal{C}\left(\Gamma_{\mathcal{K}}, \mathcal{O}_{\mathfrak{p}}\right) \rightarrow \mathcal{C}\left(\mathfrak{G}_{\mathfrak{c}}, \mathcal{O}_{\mathfrak{p}}\right), \varphi \mapsto \varphi_{\psi}$ by $\varphi_{\psi}(g)=$ $\varphi\left(\left.g\right|_{\mathcal{K}_{\infty}}\right) \psi(g)$. We let $\mathcal{E}_{\psi, \mathfrak{n}}^{\text {ord }}$ be the $p$-adic Eisenstein measure on $\Gamma_{\mathcal{K}}$ obtained by the pull-back of $\mathcal{E}_{2,1}^{\text {ord }}$ along the above morphism. In other words, we have

$$
\int_{\Gamma_{\mathcal{K}}} \varphi d \mathcal{E}_{\psi, \mathfrak{n}}^{o r d}=\int_{\mathfrak{G}_{\mathfrak{c}}} \varphi_{\psi} d \mathcal{E}_{2,1}^{o r d}
$$

By Prop. [5.14, $\mathcal{E}_{\psi, \mathfrak{n}}^{\text {ord }}$ interpolates $\widehat{E}^{\text {ord }}(\psi \epsilon \mid \mathbf{1}, \mathfrak{n})$ for $\widehat{\epsilon} \in \mathfrak{X}_{\Lambda}^{+}$. On the other hand, it follows from Lemma 5.3 (1) that

$$
[t] \cdot \widehat{E}^{\text {ord }}(\chi \mid \mathbf{1}, \mathfrak{n})=\left(\mathbf{1}, \widehat{\chi}_{\bar{w}}, \widehat{\chi}_{w}\right)_{w \in \Sigma_{p}}(t) \cdot \widehat{E}^{\text {ord }}(\chi \mid \mathbf{1}, \mathfrak{n}) \quad\left(\chi=\psi \epsilon, t \in \mathbf{T}=O_{p}^{3}\right) .
$$

Therefore, $\mathcal{E}_{\psi, \mathfrak{n}}^{\text {ord }}$ gives rise to a $\Lambda$-adic modular form $\mathcal{E}^{\text {ord }}(\Psi \mid \mathbf{1}, \mathfrak{n}) \in \mathcal{M}_{\text {ord }}(K, \Lambda)$ with the desired specialization property.

\section{Constant terms of the $p$-Adic Eisenstein series}

This section is devoted to the calculation of the constant term of the $\Lambda$-adic Eisenstein series $\mathcal{E}^{\text {ord }}(\Psi \mid \mathbf{1}, \mathfrak{n})$. The main formula is Prop. 6.6.

6.1. The constant term and Siegel $\boldsymbol{\Phi}$-operator. Let $E_{\mathbf{A}}(g):=E_{\mathbf{A}}\left(g,\left(\phi_{\chi, s}^{\Upsilon}\right)^{p b}\right)$ be the adelic Eisenstein series attached to the pull-back section $\left(\phi_{\chi, s}^{\Upsilon}\right)^{p b}$. The (adelic) constant term function of $E_{\mathbf{A}}$ along the parabolic subgroup $P$ is given by

$$
\left(E_{\mathbf{A}}\right)_{P}(g)=\int_{N_{P}(\mathcal{F}) \backslash N_{P}(\mathbf{A})} E_{\mathbf{A}}(n g) d n=\left(\phi_{\chi, s}^{\Upsilon}\right)^{p b}(g)+M\left(\left(\phi_{\chi, s}^{\Upsilon}\right)^{p b}\right)(g),
$$

where

$$
M\left(\left(\phi_{\chi, s}^{\Upsilon}\right)^{p b}\right)(g)=\int_{N_{P}(\mathbf{A})}\left(\phi_{\chi, s}^{\Upsilon}\right)^{p b}\left(w_{l} n g\right) d n .
$$

The constant term of holomorphic modular forms indeed is computed by the analytic Siegel $\Phi$-operator. Let $E_{\text {hol }}$ be the holomorphic Eisenstein series associated to $E_{\mathbf{A}}$ as in (5.19). Let $g \in G\left(\mathbf{A}_{f}\right)$. We consider the image $\Phi_{P, g}\left(E_{h o l}\right)$ of $E_{h o l}$ under the analytic Siegel $\Phi_{P, g}$-operator defined in 3.6.6. By Lemma 3.12. $\Phi_{P, g}\left(E_{h o l}\right)$ is a modular form of weight zero on $G_{P}$, i.e. a locally constant function on $G_{P}\left(\mathbf{A}_{f}\right)$. Recall that $G_{P}=G U(W) \hookrightarrow G U(V), g_{1} \mapsto m\left(\nu\left(g_{1}\right), g_{1}\right)$. Recall that

$$
E_{h o l}(\tau, g)=\left.\chi(\nu(g)) \cdot J_{k}\left(g_{\infty}, \mathbf{i}_{0}\right) E_{\mathbf{A}}\left(\left(g_{\infty}, g\right)\right)\right|_{s=0} \quad\left(g_{\infty} \mathbf{i}_{0}=\tau\right) .
$$

In particular, $E_{h o l}\left(\mathbf{i}_{0}, n_{f} g\right)=E_{\mathbf{A}}(n g) \chi(\nu(g))$ for $n=\left(n_{\infty}, n_{f}\right) \in N_{P}(\mathbf{A})$. Using (3.13), it is easy to show that

$$
\Phi_{P, g}\left(E_{h o l}\right)\left(g_{1}\right)=\left.\left(E_{\mathbf{A}}\right)_{P}\left(g_{1} g\right)\right|_{s=0} \cdot \chi\left(\nu\left(g_{1} g\right)\right) \quad\left(g_{1} \in G_{P}\left(\mathbf{A}_{f}\right)\right) .
$$

\footnotetext{
${ }^{2}$ This may be regarded as the $\Lambda$-adic weight of $F$.
} 
Lemma 6.1. If $k>2$, then $\left.M\left(\left(\phi_{\chi, s, \infty}\right)^{p b}\right)\right|_{s=0}=0$. In particular,

$$
\Phi_{P, g}(E(\chi \mid \mathbf{1}, \mathfrak{n}))\left(g_{1}\right)=\left.\frac{\Lambda_{2}^{S \cup S_{p}}(s, \chi)}{\operatorname{vol}\left(K_{P}(\mathfrak{n})\right)} \cdot\left(\phi_{\chi, s}^{\Upsilon}\right)^{p b}\left(m\left(1, g_{1}\right) g\right)\right|_{s=0} \cdot \chi(\nu(g)) .
$$

Proof. This is essentially [Hsi11, Prop. 5.3]. The proof is omitted.

6.2. The local pull-back section at $\boldsymbol{v} \in \boldsymbol{S}$. We determine $\phi_{\chi, s, v}^{p b}$ for $v \in S$ by computing the local pull-back section $f_{\mathfrak{n}, v}^{p b}$ of $f_{\mathfrak{n}, v}$ defined in $\$ 5.3 .2$

Lemma 6.2. Let $v \in S$ and let $\alpha \in U(V)\left(\mathcal{F}_{v}\right)$ and $\gamma \in U(W)\left(\mathcal{F}_{v}\right)$. Then we have

(1) $i(1, \gamma) \in Q(F) D_{\mathbf{W}}\left(\mathfrak{n}_{v}\right) \Longleftrightarrow \gamma \in D_{W}\left(\mathfrak{d}_{v} \mathfrak{n}_{v}\right)$. In this case, $f_{\mathfrak{n}, v}(i(1, \gamma))=1$.

(2) $\alpha \in \operatorname{supp} f_{\mathfrak{n}}^{p b} \Rightarrow \alpha \in P(F) D_{V}^{1}\left(\mathfrak{n}_{v}\right)$.

(3) If $\alpha \in D_{V}^{1}\left(\mathfrak{n}_{v}\right)$, then $f_{\mathfrak{n}, v}^{p b}(g)=\operatorname{vol}\left(D_{W}\left(\mathfrak{d}_{v} \mathfrak{n}_{v}\right)\right)$.

Proof. The proof is a slight refinement of Shi97, Lemma 21.4]. Write $\mathfrak{r}=\mathfrak{r}_{v}$, $\mathfrak{n}=\mathfrak{n}_{v}, \mathfrak{d}_{v}=\mathfrak{d}$ and let $F=\mathcal{F}_{v}$. By the assumption (h3), $\vartheta \mathfrak{r}=\mathfrak{d}$, so $M_{v}$ is the standard lattice and $K_{v}^{0}=\mathrm{GL}_{3}(\mathfrak{r}) \cap U(V)(F)$. We put

$$
\alpha=\left[\begin{array}{lll}
a & b & c \\
g & e & f \\
h & l & d
\end{array}\right] .
$$

Since $\alpha^{-1}=\vartheta_{3,1} \alpha^{*} \vartheta_{3,1}^{-1} \in K_{v}^{0}$, we find that

$$
\alpha^{-1}=\left[\begin{array}{ccc}
-d^{*} & f^{*} \vartheta^{-1} & -c^{*} \\
-\vartheta l^{*} & e^{*} & \vartheta b^{*} \\
-h^{*} & g^{*} \vartheta^{-1} & a^{*}
\end{array}\right] .
$$

In particular, $\alpha^{-1} \in D_{V}^{1}(\mathfrak{n})$ if $\alpha \in D_{V}^{1}(\mathfrak{n})$, and $D_{V}^{1}(\mathfrak{n})$ is a group. Now we prove part (1). First the assumption (a1) implies that

$$
8^{-1} \mathfrak{n} \subsetneq \mathfrak{c}_{v} .
$$

Using (5.16), we have

$$
i(1, \gamma)=\Delta^{-1}\left[\begin{array}{ll}
1 & 0 \\
0 & \gamma
\end{array}\right] \Delta=\left[\begin{array}{cccc}
1 & & & \\
& \frac{1}{2}(1+\gamma) & & \frac{\vartheta}{4}(1-\gamma) \\
& & 1 & \\
\vartheta^{-1}(1-\gamma) & & \frac{1}{2}(1+\gamma)
\end{array}\right]
$$

By [Shi97, Lemma 9.2], we find that

$$
\begin{aligned}
i(1, \gamma) \in Q(F) D_{\mathbf{W}}(\mathfrak{n}) & \Longleftrightarrow 2(1+\gamma)^{-1} \vartheta^{-1}(1-\gamma) \in \mathfrak{n} \\
& \Longleftrightarrow i(1, \gamma) \prec\left[\begin{array}{cc}
1+2^{-1} \mathfrak{d} \mathfrak{n} & \mathfrak{r} \\
\mathfrak{n} & 1+2^{-1} \mathfrak{d} \mathfrak{n}
\end{array}\right] \\
& \Longleftrightarrow \gamma-1 \prec \mathfrak{d} \mathfrak{n} \text { and } f_{\mathfrak{n}, v}(i(1, \gamma))=1 .
\end{aligned}
$$

This completes the proof of part (1).

We proceed to prove part (2) and part (3). By Iwasawa decomposition we may assume $\alpha \in K_{v}^{0}$ and $i\left(\alpha, \gamma_{1}\right) \in Q(F) D_{\mathbf{W}}(\mathfrak{n})$ for some $\gamma_{1} \in U(W)(F)$. Then $\alpha^{-1} \prec \mathfrak{r}$, and by (6.2) we have

$$
[g, f] \prec \mathfrak{d} ; \frac{1}{2} f^{*} \vartheta f=f^{*} \vartheta^{-1}\left(\frac{1}{2} \vartheta\right) \vartheta^{-1} f \in 2^{-1} \mathfrak{d} .
$$


Put

$$
\alpha_{1}:=\left[\begin{array}{ccc}
1 & -f^{*} \vartheta^{-1} & \frac{1}{2} f^{*} \vartheta^{-1} f \\
& 1 & -f \\
& & 1
\end{array}\right]\left[\begin{array}{ccc}
d^{*} & 0 & 0 \\
& 1 & 0 \\
& & d^{-1}
\end{array}\right]\left[\begin{array}{lll}
a & b & c \\
g & e & f \\
h & l & d
\end{array}\right]=\left[\begin{array}{ccc}
a_{1} & b_{1} & c_{1} \\
g_{1} & e_{1} & 0 \\
h_{1} & l_{1} & 1
\end{array}\right] .
$$

Then $\alpha_{1} \in U(V)(F)$. Since $i\left(m\left(1, \gamma_{1}^{-1}\right) \alpha, 1\right) \in Q(F) D_{\mathbf{W}}(\mathfrak{n})$, by [Shi97, (21.4.4)], we have

$$
\begin{aligned}
{\left[\begin{array}{cc}
h_{1} & l_{1} \\
\vartheta^{-1} \gamma_{1}^{-1} g_{1} & \vartheta^{-1}\left(\gamma_{1}^{-1} e_{1}-1\right)
\end{array}\right] } & =\left[\begin{array}{cc}
d^{-1} h & d^{-1} l \\
\vartheta^{-1} \gamma_{1}^{-1}\left(g-f d^{-1} h\right) & \vartheta^{-1}\left(\gamma_{1}^{-1}\left(e-f d^{-1} l\right)-1\right)
\end{array}\right] \\
& \prec \mathfrak{n} .
\end{aligned}
$$

In addition, from (6.3) we deduce that

$$
\left[a_{1}, b_{1}\right] \prec \mathfrak{r}, c_{1} \in 2^{-1} \mathfrak{r} .
$$

The equation $\alpha_{1} \vartheta_{2,1} \alpha_{1}^{*}=\vartheta_{2,1}$ implies that

$$
e_{1} \vartheta e_{1}^{*}=\vartheta, c_{1} g_{1}^{*}+b_{1} \vartheta e_{1}^{*}=0, c_{1} h_{1}^{*}+b_{1} \vartheta l_{1}^{*}-a_{1}=-1 .
$$

Putting these together, we find that

$$
\gamma_{1}^{-1} e_{1} \in 1+\mathfrak{d n}, b_{1} \in 2^{-1} \mathfrak{n}, a-1 \in 2^{-1} \mathfrak{n} .
$$

Put

$$
\alpha_{2}=\left[\begin{array}{ccc}
1 & 0 & -\frac{1}{2}\left(c_{1}+c_{1}^{*}\right) \\
& \gamma_{1}^{-1} & 0 \\
& & 1
\end{array}\right] \alpha_{1}=\left[\begin{array}{ccc}
a_{2} & b_{2} & c_{2} \\
g_{2} & e_{2} & 0 \\
h_{1} & l_{1} & 1
\end{array}\right] \in U(V)(F) .
$$

Then we have $c_{2}^{*}+c_{2}=0, a_{2} \in 1+4^{-1} \mathfrak{n}, b_{2} \in 4^{-1} \mathfrak{n}, g_{2} \in \mathfrak{d n}, e_{2} \in \mathfrak{r}^{\times}$and

$$
c_{2}\left(a_{2}+a_{2}^{*}\right)=b_{2} \vartheta b_{2}, a_{2}=c_{2} h_{1}^{*}+b_{2} \vartheta l_{1}^{*}+1 .
$$

It follows that $a_{2}+a_{2}^{*} \in 2 \mathfrak{r}^{\times}, c_{2} \in 32^{-1} \mathfrak{n}^{2} \subset \mathfrak{r}, a_{2} \in 1+\mathfrak{n}$. Hence $\alpha_{2} \in D_{V}^{1}(\mathfrak{n})$, and part (2) follows.

If $\alpha \in D_{V}^{1}(\mathfrak{n})$, the computation in the proof of (2) shows that there exists $\beta \in$ $N_{P}\left(4^{-1} \mathfrak{r}\right)$ such that

$$
\alpha_{2}=\beta \cdot m\left(d^{-1}, 1\right) \alpha \in\left[\begin{array}{ccc}
1+\mathfrak{n} & \mathfrak{n} & \mathfrak{r} \\
\mathfrak{d n} & \mathfrak{r}^{\times} & 0 \\
\mathfrak{n} & \mathfrak{n} & 1
\end{array}\right], d \in 1+\mathfrak{n} .
$$

Then $\left(\alpha_{2}, e_{2}\right)_{\Delta} \in D_{\mathbf{W}}(\mathfrak{n})$. Thus

$$
\begin{aligned}
f_{\mathfrak{n}, v}^{p b}(\alpha) & =\int_{U(W)(F)} f_{\mathfrak{n}, v}\left(i\left(\alpha_{2}, h\right)\right) \chi(h) d h \\
& =\int_{U(W)(F)} f_{\mathfrak{n}, v}\left(i\left(1, h e_{2}^{-1}\right)\right) \chi^{-1}\left(e_{2}\right) \chi(h) d h \\
& =\int_{U(W)(F)} f_{\mathfrak{n}, v}(i(1, h)) \chi(h) d h=\operatorname{vol}\left(D_{W}(\mathfrak{d n})\right) \quad(B y(1)) .
\end{aligned}
$$

This completes the proof of part (3).

Proposition 6.3. If $v \in S$, then $\phi_{\chi, s, v}^{p b}=\operatorname{vol}\left(D_{W}\left(\mathfrak{d}_{v} \mathfrak{n}_{v}\right)\right) \cdot f_{\chi, \mathbf{1}, s, v}^{\mathfrak{n}}$, where $f_{\chi, \mathbf{1}, s, v}^{\mathfrak{n}}$ is the unique section supported in $P(F) w_{l} D_{V}\left(\mathfrak{n}_{v}\right)$ such that

$$
f_{\chi, \mathbf{1}, s, v}^{\mathfrak{n}}\left(w_{l} u\right)=1 \text { for every } u \in D_{V}\left(\mathfrak{n}_{v}\right) .
$$


Proof. Note that $i\left(w_{l}, 1\right)=\mathbf{w}_{l}^{\prime}$. We have

$$
\phi_{\chi, s, v}^{p b}(g)=\int_{U(W)(F)} f_{\mathfrak{n}, v}\left(i\left(g w_{l}, h\right)\right) \chi(h) d h=f_{\mathfrak{n}, v}^{p b}\left(g w_{l}\right) .
$$

by the definition of $\phi_{\chi, s, v}$. Then (2) can be easily deduced from Lemma 6.2.

\subsection{The ordinary projection of the local sections at $p$.}

6.3.1. The ordinary linear functional. Let $v$ be a place above $p$ and let $d_{v}=\left[\mathcal{F}_{v}\right.$ : $\mathbf{Q}_{p}$ ] be the degree of $v$ over $\mathbf{Q}$. Let $F=\mathcal{F}_{v}$ and $\mathcal{O}=\mathcal{O}_{F}$. In this subsection, we shall retain the notation in $\$ 5.3 .4$. Let $w$ be the place in $\Sigma_{p}$ lying above $v$ and let $\left(\chi_{1}, \chi_{2}\right)=\left(\chi_{w}, \chi_{\bar{w}}\right)$ be characters on $F^{\times}$. Let $\Phi_{v}$ be the Bruhat-Schwartz function on $M_{2 \times 4}(F)$ in (5.7), and let $\Phi_{v}^{\Upsilon}(x):=\Phi_{v}(x \Upsilon)$. Then the section $\phi_{\chi, s, v}^{\Upsilon}$ is the Godement-Jacquet section $f_{\Phi_{v}^{\Upsilon}, v}$ associated to $\Phi_{v}^{\Upsilon}$ defined in (5.8). Write $f_{\Phi^{\Upsilon}}$ for $f_{\Phi_{v}^{\Upsilon}, v}$. We shall calculate the ordinary projection $e_{v} \cdot\left(\phi_{\chi, 0, v}^{\Upsilon}\right)^{p b}$ of the pull-back section $\phi_{\chi, 0, v}^{p b}=\left.\operatorname{vol}\left(\mathcal{P}_{2, v}\right)^{-1} \cdot f_{\Phi \Upsilon}^{p b}\right|_{s=0}$, using the technique of the ordinary linear functional in Hsi11, $\S 6$.

For a triple $\left(\lambda_{1}, \lambda_{2}, \lambda_{3}\right)$ of characters of $F^{\times}$, let $I\left(\lambda_{1}, \lambda_{2}, \lambda_{3}\right)$ denote the unitarily induced representation of $\mathrm{GL}_{3}(F)$ from the the character $\lambda$ of $B_{3}(F)$ given by

$$
\lambda\left(\left[\begin{array}{lll}
a & * & * \\
& b & * \\
& & c
\end{array}\right]\right)=\lambda_{1}(a) \lambda_{2}(b) \lambda_{3}(c) .
$$

We say $I\left(\lambda_{1}, \lambda_{2}, \lambda_{3}\right)$ is regular if the $p$-adic valuations of $\left\{\lambda_{i}(p)\right\}_{i=1,2,3}$ are distinct. Via the identification $G(F)=\mathrm{GL}_{3}(F) \times F^{\times}$in (1.2), we have an isomorphism:

$$
I_{v}(\chi, \mathbf{1}, 0)=I\left(\chi_{2}|\cdot|^{-1}, \mathbf{1}, \chi_{1}^{-1}|\cdot|\right) \otimes \chi_{2}^{-1} .
$$

In other words, $I_{v}(\chi, \mathbf{1}, 0)$ is identified with the space consisting of smooth functions $f: \mathrm{GL}_{3}(F) \rightarrow \mathbf{C}$ such that

$$
f(t n g)=\left(\chi_{2}, \mathbf{1}, \chi_{1}^{-1}\right)(t) f(g) \text { for } t \in T_{3}(F), n \in N_{3}(F),
$$

on which $\mathrm{GL}_{3}(F) \times F^{\times}$acts by $((g, \nu) f)(h)=f(h g) \chi_{2}^{-1}(\nu)$. We have $v_{p}\left(\chi_{1}(p)\right)=$ $-k d_{v}$ and $v_{p}\left(\chi_{2}(p)\right)=0$ since the infinity type of $\chi$ is $k \Sigma$. Thus the representation $I\left(\chi_{2}|\cdot|^{-1}, \mathbf{1}, \chi_{1}^{-1}|\cdot|\right)$ is regular $(k>2)$. Introduce Weyl elements

$$
w_{2}=\left[\begin{array}{lll} 
& 1 & \\
1 & & \\
& & 1
\end{array}\right], s_{1}=\left[\begin{array}{lll} 
& & 1 \\
1 & & \\
& 1 &
\end{array}\right] \text { and } w_{3}=\left[\begin{array}{lll} 
& 1 & \\
1 & &
\end{array}\right] \in \mathrm{GL}_{3}(F) .
$$

Let $f^{\dagger} \in I\left(\chi_{1}^{-1}|\cdot|, \chi_{2}|\cdot|^{-1}, \mathbf{1}\right) \otimes \chi_{2}^{-1}$ be the unique function such that

$$
\operatorname{supp} f^{\dagger}=B_{3}(F) w_{3} N_{3}(\mathcal{O}) \text { and } f^{\dagger}\left(w_{3} n\right)=1 \text { for all } n \in N_{3}(\mathcal{O}) \text {. }
$$

Let $\alpha=\left(\operatorname{diag}\left(p^{a_{1}}, p^{a_{2}}, p^{a_{3}}\right), p^{a_{3}}\right) \in \mathrm{GL}_{3}(F) \times F^{\times}=G(F)$ with integers $a_{1} \leq a_{2} \leq$ $a_{3}$. The normalized $U_{v}(\alpha)$-operators on $I_{v}(\chi, \mathbf{1}, 0)$ with respect to the weight $\underline{k}=$ $(0,0 ; k \Sigma)$ are given by 


$$
\begin{aligned}
U_{v}(\alpha) \cdot f(g)= & \frac{\chi_{2}\left(p^{-a_{3}}\right)}{p^{2 a_{3}-a_{1}-a_{2}}} \sum_{\substack{y_{1} \in \mathcal{O} /\left(p^{a_{2}-a_{1}}\right), y_{2} \in \mathcal{O} /\left(p^{a_{3}-a_{1}}\right) \\
, y_{3} \in \mathcal{O} /\left(p^{a_{3}-a_{2}}\right)}} \\
& \times f\left(g\left[\begin{array}{ccc}
1 & y_{1} & y_{2} \\
0 & 1 & y_{3} \\
0 & 0 & 1
\end{array}\right]\left[\begin{array}{lll}
p^{a_{3}-a_{1}} & \\
& p^{a_{3}-a_{2}} & \\
& & 1
\end{array}\right]\right) .
\end{aligned}
$$

A straightforward computation shows that $U_{v}(\alpha) \cdot f^{\dagger}$ is right invariant by $N_{3}(\mathcal{O})$ and is supported in the big cell $B_{3}(F) w_{3} N_{3}(\mathcal{O})$ (use $\alpha^{-1} N_{3}(\mathcal{O}) \alpha \subset N_{3}(\mathcal{O})$ ). It follows that $f^{\dagger}$ is an eigenvector of the operator $U_{v}(\alpha)$ with the eigenvalue $U_{v}(\alpha) \cdot f^{\dagger}\left(w_{3}\right)=$ $\chi_{2}\left(p^{-a_{2}}\right)$, which is a $p$-adic unit. In other words, $f^{\dagger}$ is ordinary. We define

$$
f_{v}^{\text {ord }}:=M_{s_{1}} f^{\dagger} \in I\left(\chi_{2}|\cdot|^{-1}, \mathbf{1}, \chi_{1}^{-1}|\cdot|\right)^{N_{3}(\mathcal{O})},
$$

where $M_{s_{1}}$ is the intertwining operator attached to the Weyl element $s_{1}$. Then $f_{v}^{\text {ord }}$ is the ordinary section such that $f_{v}^{\text {ord }}\left(w_{2}\right)=1$. For $x \in F$, we set

$$
u(x)=\left[\begin{array}{lll}
1 & x & \\
& 1 & \\
& & 1
\end{array}\right] \in N_{3}(F) .
$$

Define the functional $l_{w_{2}}: I\left(\chi_{2}|\cdot|^{-1}, \mathbf{1}, \chi_{1}^{-1}|\cdot|\right)^{N_{3}(\mathcal{O})} \rightarrow \mathbf{C}$ by

$$
f \rightarrow l_{w_{2}}(f):=M_{w_{2}}(f)(1)=\int_{F} f\left(w_{2} u(x)\right) d x .
$$

The integral $l_{w_{2}}(f)$ converges absolutely if $k>1$, and $l_{w_{2}}$ is the ordinary functional on the regular representation in the sense of [Hsi11, §6.2.1]. Let $U_{v}:=U_{v}(\alpha)$ with $\left(a_{1}, a_{2}, a_{3}\right)=(1,2,3)$ and $e_{v}:=\lim _{n \rightarrow \infty} U_{v}^{n !}$.

Lemma 6.4. For $f \in I\left(\chi_{2}|\cdot|^{-1}, \mathbf{1}, \chi_{1}^{-1}|\cdot|\right)^{N_{3}(\mathcal{O})}$, the ordinary projection $e_{v} \cdot f$ is given by

$$
e_{v} . f=l_{w_{2}}(f) f_{v}^{\text {ord }} \text {. }
$$

Proof. This follows from the same argument in [Hsi11, Lemma 6.4].

By Lemma 5.2 $f_{\Phi}^{p b}$ is $N_{3}(\mathcal{O})$-invariant, and hence by Lemma 6.4 we find that

$$
e_{v} \cdot\left(\phi_{\chi, 0, v}^{\Upsilon}\right)^{p b}=\left.\frac{1}{\operatorname{vol}\left(\mathcal{P}_{2, v}\right)} \cdot e_{v} \cdot f_{\Phi \Upsilon}^{p b}\right|_{s=0}=\left.\frac{1}{\operatorname{vol}\left(\mathcal{P}_{2, v}\right)} \cdot l_{w_{2}}\left(f_{\Phi}^{p b}\right)\right|_{s=0} \cdot f_{v}^{\text {ord }} .
$$

6.3.2. The calculation of the ordinary projection $e_{v} \cdot f_{\Phi^{\Upsilon}}^{p b}$. We proceed to compute $l_{w_{2}}\left(f_{\Phi}^{p b}\right)$. By the definition (6.5) of $l_{w_{2}}$, we have

$$
\left.l_{w_{2}}\left(f_{\Phi^{\Upsilon}}^{p b}\right)=\int_{F} d x \int_{F^{\times}} d h f_{\Phi_{v}, v}\left(i\left(w_{2} u(x), h\right)\right) \Upsilon\right) \chi_{1} \chi_{2}^{-1}(h),
$$


where we have used the identification $F^{\times}=U(W)(F)$ in (5.17). By a direct computation, we find that

$$
\begin{aligned}
\left.(0, Z) \cdot i\left(w_{2} u(x), h\right)\right) \Upsilon & =(0, Z) \cdot \Delta^{-1}\left[\begin{array}{ccc}
w_{2} u(x) & 0 \\
0 & h
\end{array}\right] \Delta \Upsilon \\
& =(0, Z) \cdot\left[\begin{array}{llll}
1 & & \\
& \frac{1}{2} & & -\frac{1}{2} \\
& 1 & \\
& 1 & & 1
\end{array}\right]\left[\begin{array}{llll}
0 & 1 & & \\
1 & x & & \\
& & 1 & 0 \\
& 0 & h
\end{array}\right] \\
& =\left(Z\left[\begin{array}{ll}
0 & 0 \\
1 & x
\end{array}\right], Z\left[\begin{array}{ll}
1 & 0 \\
0 & h
\end{array}\right]\right) .
\end{aligned}
$$

We thus have

$$
l_{w_{2}}\left(f_{\Phi}^{p b}\right)=\int_{F} d x \int_{F} d h \int_{\mathrm{GL}_{2}(F)} d^{\times} Z P(h) Q(Z) \Phi_{v}\left(Z\left[\begin{array}{ll}
0 & 0 \\
1 & x
\end{array}\right], Z\left[\begin{array}{ll}
1 & 0 \\
0 & h
\end{array}\right]\right),
$$

where $P(h)=\chi_{1}(h)|h|^{s}$ and $Q(Z)=\chi_{1} \chi_{2}(\operatorname{det} Z)|\operatorname{det} Z|^{2 s}$. We make a change of variable

$$
Z \mapsto Z\left[\begin{array}{cc}
1 & 0 \\
0 & x^{-1}
\end{array}\right] ; h \mapsto x h,
$$

so the integral (6.7) equals

$$
\begin{aligned}
& \int_{F} \chi_{2}^{-1}(x)|x|_{v}^{-s} d x \int_{F} P(h) d h \\
& \quad \times \int_{\mathrm{GL}_{2}(F)} Q(Z) \Phi_{\bar{w}}\left(Z\left[\begin{array}{cc}
0 & 0 \\
x^{-1} & 1
\end{array}\right]\right) \widehat{\Phi}_{w}\left(Z\left[\begin{array}{cc}
1 & 0 \\
0 & h
\end{array}\right]\right) d^{\times} Z .
\end{aligned}
$$

Consider the last integral against $Z$ and write $Z=\left[\begin{array}{ll}Z_{1} & Z_{2} \\ Z_{3} & Z_{4}\end{array}\right]$. Then we have (6.9)

$$
\Phi_{\bar{w}}\left(Z\left[\begin{array}{cc}
0 & 0 \\
x^{-1} & 1
\end{array}\right]\right) \widehat{\Phi}_{w}\left(Z\left[\begin{array}{ll}
1 & 0 \\
0 & h
\end{array}\right]\right)=\Phi_{\bar{w}}\left(\left[\begin{array}{cc}
Z_{2} x^{-1} & Z_{2} \\
Z_{4} x^{-1} & Z_{4}
\end{array}\right]\right) \widehat{\Phi}_{w}\left(\left[\begin{array}{cc}
Z_{1} & Z_{2} h \\
Z_{3} & Z_{4} h
\end{array}\right]\right) .
$$

In particular, $Z_{4} \in \mathcal{O}^{\times}$, so we can write

$$
Z=\left[\begin{array}{ll}
Z_{1} & Z_{2} \\
Z_{3} & Z_{4}
\end{array}\right]=\left[\begin{array}{ll}
1 & y \\
0 & 1
\end{array}\right]\left[\begin{array}{cc}
a & 0 \\
Z_{3} & Z_{4}
\end{array}\right]
$$

We then decompose

$$
d^{\times} Z=|\operatorname{det} Z|^{-2} d Z=|a|^{-1} d^{\times} a d y d Z_{3} d^{\times} Z_{4}
$$

and $\operatorname{vol}\left(\mathcal{P}_{2, v}\right)=\left|\varpi^{m_{v}}\right|$. Taking into account the supports of $\Phi_{\bar{w}}$ and $\Phi_{w}$ in (6.9), we find that

$$
Z_{3} \in \mathcal{O}, y \in \mathcal{O}
$$


By (5.6), the last integral in (6.8) equals

$$
\begin{gathered}
\int_{F^{\times}} d^{\times} a \int_{\mathcal{O}} d y \int_{\mathcal{O}^{\times}} d^{\times} Z_{4} \int_{\mathcal{O}} d Z_{3} \chi_{+}(a)|a|^{2 s-1} \Phi_{\bar{w}}\left(\left[\begin{array}{cc}
0 & 0 \\
x^{-1} & 1
\end{array}\right]\right) \widehat{\varphi}_{\nu_{1}}(a) \widehat{\varphi}_{\nu_{2}}(h)\left|\varpi^{m_{v}}\right| \\
=\left|\varpi^{m_{v}}\right| \operatorname{Eul}\left(2 s-1, \nu_{1}\right) \widehat{\varphi}_{\nu_{2}}(h) \Phi_{\bar{w}}\left(\left[\begin{array}{cc}
0 & 0 \\
x^{-1} & 1
\end{array}\right]\right),
\end{gathered}
$$

where we adopted the notation in Def. 5.1, so the integral (6.8) equals

$$
\left|\varpi^{m_{v}}\right| \operatorname{Eul}\left(2 s-1, \nu_{1}\right) \int_{F} \chi_{2}^{-1}(x)|x|^{-s} \Phi_{\bar{w}}\left(\left[\begin{array}{cc}
0 & 0 \\
x^{-1} & 1
\end{array}\right]\right) d x \cdot \int_{F} P(h) \widehat{\varphi}_{\nu_{2}}(h) d h .
$$

A straightforward computation shows that

$$
\int_{F} P(h) \widehat{\varphi}_{\nu_{2}}(h) d h=\operatorname{Eul}\left(s, \nu_{2}\right)(\text { by }(\underline{5.6}))
$$

and

$$
\begin{aligned}
\int_{F} \chi_{2}^{-1}(x)|x|^{-s} \Phi_{\bar{w}}\left(\left[\begin{array}{cc}
0 & 0 \\
x^{-1} & 1
\end{array}\right]\right) d x & =\chi_{2}(\varpi)|\varpi|^{s} \operatorname{vol}\left(\varpi^{-1} \mathcal{O}^{\times}, d x\right) \\
& =\chi_{2}(\varpi)|\varpi|^{s}\left(|\varpi|^{-1}-1\right) .
\end{aligned}
$$

Combining the above equations with (6.8) and rearranging terms, finally we obtain

$$
l_{w_{2}}\left(f_{\Phi^{\Upsilon}}^{p b}\right)=\operatorname{vol}\left(\mathcal{P}_{2, v}\right)\left(p^{d_{v}}-1\right) \cdot \chi_{2}|\cdot|^{s}(\varpi) \cdot \operatorname{Eul}\left(2 s-1, \chi_{+}\right) \cdot \operatorname{Eul}\left(s, \chi_{1}\right) .
$$

6.4. Constant terms of $\boldsymbol{e} \cdot \boldsymbol{E}(\chi \mid \mathbf{1}, \mathfrak{n})$. Now we are ready to give the precise formula for the pull-back section and calculate the constant term of the ordinary projection $e . E(\chi \mid \mathbf{1}, \mathfrak{n})$ of the Eisenstein series $E(\chi \mid \mathbf{1}, \mathfrak{n})$. Comparing (6.4) with Prop. $3.15(\underline{k}(\alpha)=1)$, we find that $e=\prod_{v \mid p} e_{v}$. By (5.18), the ordinary Eisenstein series e.E $(\chi \mid \mathbf{1}, \mathfrak{n})$ is associated to the normalized $p$-ordinary section $\left(f_{\chi, \mathbf{1}}^{\text {ord }}\right)^{*} \in$ $I(\chi, \mathbf{1}, 0)$ given by

$$
\left(f_{\chi, \mathbf{1}}^{\text {ord }}\right)^{*}:=\left.\frac{\Lambda_{2}^{S \cup S_{p}}(s, \chi)}{\operatorname{vol}\left(K_{P}(\mathfrak{n})\right)} \cdot\left(\phi_{\chi, s}^{\{p\}}\right)^{p b} \bigotimes_{v \in S_{p}} e_{v} \cdot\left(\phi_{\chi, s, v}^{\Upsilon}\right)^{p b}\right|_{s=0} .
$$

By Prop. 5.11 and Prop. 6.3, we find that

$$
\begin{aligned}
\left(f_{\chi, \mathbf{1}}^{\text {ord }}\right)^{*}= & f_{\chi, \mathbf{1}}^{\text {ord }} \cdot\left(2^{-1}(-1)^{k} \Gamma_{\mathbf{C}}(k) \Gamma_{\mathbf{C}}(k-1)\right)^{d} \cdot L^{S \cup S_{p}}\left(-1, \chi_{+} \tau_{\mathcal{K} / \mathcal{F}}\right) \cdot L^{S \cup S_{p}}(0, \chi) \\
& \times\left.\operatorname{vol}\left(\mathcal{P}_{2, v}\right)^{-1} l_{w_{2}}\left(f_{\Phi \Upsilon}^{p b}\right)\right|_{s=0},
\end{aligned}
$$

where $d=[\mathcal{F}: \mathbf{Q}]$ and

$$
f_{\chi, \mathbf{1}}^{\text {ord }}=f_{k, \infty} \bigotimes_{v \notin S \cup\{p\}} f_{\chi, \mathbf{1}, 0, v}^{0} \bigotimes_{v \in S} f_{\chi, \mathbf{1}, 0, v}^{\mathfrak{n}} \bigotimes_{v \in S_{p}} f_{v}^{\text {ord }} .
$$

From (6.6) and the formula (6.12) for $l_{w_{2}}\left(f_{\Phi \Upsilon}^{p b}\right)$ we deduce that

$$
\begin{aligned}
\left(f_{\chi, \mathbf{1}}^{\text {ord }}\right)^{*}= & f_{\chi, \mathbf{1}}^{\text {ord }} \cdot\left((-2 i) \cdot \frac{\Gamma(k) \Gamma(k-1)}{(2 \pi i)^{(2 k-1)}}\right)^{d} \cdot L^{S \cup S_{p}}\left(-1, \chi_{+} \tau_{\mathcal{K} / \mathcal{F}}\right) L^{S \cup S_{p}}(0, \chi) \\
& \times \prod_{w \in \Sigma_{p}, w \mid v} \operatorname{Eul}\left(-1, \chi_{+, v}\right) \operatorname{Eul}\left(0, \chi_{w}\right) \chi_{w}(p)\left(p^{d_{v}}-1\right) .
\end{aligned}
$$


Define the $(\infty, p)$-modified $L$-values by

$$
\begin{aligned}
L_{(\infty, p)}^{S}(0, \chi) & =\frac{\left[\mathcal{O}_{\mathcal{K}}^{\times}: \mathcal{O}_{\mathcal{F}}^{\times}\right]}{\sqrt{\left|D_{\mathcal{F}}\right|_{\mathbf{R}}}} \cdot \frac{\Gamma(k)^{d}}{(2 \pi i)^{k d}} \cdot \prod_{w \in \Sigma_{p}} \operatorname{Eul}\left(0, \chi_{w}\right) \cdot L^{S \cup S_{p}}(0, \chi) ; \\
L_{(\infty, p)}^{S}\left(-1, \chi_{+} \tau_{\mathcal{K} / \mathcal{F}}\right) & =\frac{\Gamma(k-1)^{d}}{(2 \pi i)^{(k-1) d}} \cdot \prod_{v \in S_{p}} \operatorname{Eul}\left(-1, \chi_{+, v}\right) \cdot L^{S \cup S_{p}}\left(-1, \chi_{+} \tau_{\mathcal{K} / \mathcal{F}}\right) .
\end{aligned}
$$

Here $L^{S \cup S_{p}}(-)$ denotes the imprimitive $L$-value obtained by removing Euler factors at places dividing those in $S \cup S_{p}$. Summarizing our calculations of local pull-back sections, we obtain the following formulas of constant terms in view of Lemma 6.1

Proposition 6.5. The ordinary Eisenstein series e.E $(\chi \mid \mathbf{1}, \mathfrak{n})$ is associated to the section

$$
\left(f_{\chi, \mathbf{1}}^{\text {ord }}\right)^{*}=f_{\chi, \mathbf{1}}^{\text {ord }} \cdot L_{(\infty, p)}^{S}(0, \chi) L_{(\infty, p)}^{S}\left(-1, \chi_{+} \tau_{\mathcal{K} / \mathcal{F}}\right) \cdot \chi_{\Sigma_{p}^{c}}(p) c_{\mathcal{K}}(p),
$$

where $c_{\mathcal{K}}(p)$ is the constant

$$
c_{\mathcal{K}}(p)=(-2 i)^{d} \cdot\left|D_{\mathcal{F}}\right|_{\mathbf{R}}^{\frac{1}{2}}\left[\mathcal{O}_{\mathcal{K}}^{\times}: \mathcal{O}_{\mathcal{F}}^{\times}\right]^{-1} \prod_{v \in S_{p}}\left(p^{d_{v}}-1\right) \in \overline{\mathbf{Z}}_{(p)}^{\times} .
$$

Therefore, we have

$$
\Phi_{P, g}(e . E(\chi \mid \mathbf{1}, \mathfrak{n}))=f_{\chi, \mathbf{1}}^{\text {ord }}(g) \cdot L_{(\infty, p)}^{S}(0, \chi) L_{(\infty, p)}^{S}\left(-1, \chi_{+} \tau_{\mathcal{K} / \mathcal{F}}\right) \cdot \chi_{\Sigma_{p}^{c}}(p) c_{\mathcal{K}}(p) .
$$

6.5. Constant terms of $\widehat{\boldsymbol{E}}^{\text {ord }}(\chi \mid \mathbf{1}, \mathfrak{n})$ and $\boldsymbol{p}$-adic $\boldsymbol{L}$-functions. We retain the notation in $\$ 5.7$. Let $\mathfrak{c}(\psi)$ and $\mathfrak{c}_{+}$be the prime-to- $p$ conductors of $\psi$ and $\psi_{+} \tau_{\mathcal{K} / \mathcal{F}}$ respectively. It follows from [Kat78, and [HT93, page 193, Thm. II] ( $c f$. Hsi13, Prop. 4.9]) that to $(\psi, \Sigma)$ we can associate a $(d+1)$-variable primitive $p$-adic Hecke $L$-function $L(\Psi, \Sigma) \in \Lambda$ such that for every $\widehat{\epsilon} \in \mathfrak{X}_{\Lambda}^{+}$with $\chi=\psi \epsilon$ of infinity type $k \Sigma$, we have

$$
\frac{1}{\Omega_{p}^{k \Sigma}} \cdot \widehat{\epsilon}\left(L_{p}(\Psi, \Sigma)\right)=\frac{\left[\mathcal{O}_{\mathcal{K}}^{\times}: \mathcal{O}_{\mathcal{F}}^{\times}\right]}{\sqrt{\left|D_{\mathcal{F}}\right|_{\mathbf{R}}}} \cdot \Gamma(k)^{d} \cdot \prod_{w \in \Sigma_{p}} \operatorname{Eul}\left(0, \chi_{w}\right) \cdot \frac{L^{S_{p}}(0, \chi)}{\Omega_{\infty}^{k \Sigma}} .
$$

On the other hand, by $\left[\mathrm{DR} 80\right.$ there exists a $p$-adic $L$-function $L_{p}\left(-1, \Psi_{+} \tau_{\mathcal{K} / \mathcal{F}}\right) \in$ Frac $\Lambda$ such that for every $\widehat{\epsilon} \in \mathfrak{X}_{\Lambda}^{+}$with $\chi=\psi \epsilon$, we have

$$
\begin{aligned}
\widehat{\epsilon}\left(L_{p}\left(-1, \Psi_{+} \tau_{\mathcal{K} / \mathcal{F}}\right)\right) & =L^{S_{p}}\left(2, \chi_{+}^{-1} \tau_{\mathcal{K} / \mathcal{F}}\right) \\
& =\frac{\Gamma(k-1)^{d}}{(2 \pi i)^{(k-1) d}} \cdot \prod_{v \in S_{p}} \operatorname{Eul}\left(-1, \chi_{+, v}\right) \cdot L^{S_{p}}\left(-1, \chi_{+} \tau_{\mathcal{K} / \mathcal{F}}\right) .
\end{aligned}
$$

Here the second equality follows from the functional equation of the complex $L$ function $L\left(s, \chi_{+} \tau_{\mathcal{K} / \mathcal{F}}\right)$. Define the $S$-imprimitive $p$-adic $L$-functions by

$$
\begin{gathered}
L_{p}^{S}(\Psi, \Sigma)=L_{p}(\Psi, \Sigma) \cdot \prod_{w \mid \mathfrak{n}, w \nmid \mathfrak{c}(\psi)}\left(1-\Psi\left(\operatorname{Frob}_{w}\right)\right) \in \Lambda . \\
L_{p}^{S}\left(-1, \Psi_{+} \tau_{\mathcal{K} / \mathcal{F}}\right)=L_{p}\left(-1, \Psi_{+} \tau_{\mathcal{K} / \mathcal{F}}\right) \cdot \prod_{v \mid \mathfrak{n}, v \nmid \mathfrak{c}_{+}}\left(1-\Psi_{+} \tau_{\mathcal{K} / \mathcal{F}}\left(\operatorname{Frob}_{v}\right)\left|\varpi_{v}\right|^{-1}\right) .
\end{gathered}
$$


Note that $L_{p}^{S}\left(-1, \Psi_{+} \tau_{\mathcal{K} / \mathcal{F}}\right)$ has no pole since $S$ is not empty. In other words, $L_{p}^{S}\left(-1, \Psi_{+} \tau_{\mathcal{K} / \mathcal{F}}\right) \in \Lambda$. By definition, the $p$-adic $L$-functions $L_{p}^{S}(\Psi, \Sigma)$ and $L_{p}^{S}\left(-1, \Psi_{+} \tau_{\mathcal{K} / \mathcal{F}}\right)$ are characterized by the specialization property at $\widehat{\epsilon} \in \mathfrak{X}_{\Lambda}^{+}$:

$$
\begin{aligned}
\frac{1}{\Omega_{p}^{k \Sigma}} \cdot \widehat{\epsilon}\left(L_{p}^{S}(\Psi, \Sigma)\right) & =\frac{(2 \pi i)^{k d}}{\Omega_{\infty}^{k \Sigma}} \cdot L_{(\infty, p)}^{S}(0, \psi \epsilon), \\
\widehat{\epsilon}\left(L_{p}^{S}\left(-1, \Psi_{+} \tau_{\mathcal{K} / \mathcal{F}}\right)\right) & =L_{(\infty, p)}^{S}\left(-1, \psi_{+} \epsilon_{+} \tau_{\mathcal{K} / \mathcal{F}}\right) .
\end{aligned}
$$

The following proposition shows the constant term of the $\Lambda$-adic Eisenstein series $\mathcal{E}^{\text {ord }}(\Psi \mid \mathbf{1}, \mathfrak{n})$ in Cor. 5.15 is essentially a product of Deligne-Ribet and Katz $p$-adic $L$-functions.

Proposition 6.6. Let $g^{0}=\left(g_{v}^{0}\right) \in G\left(\mathbf{A}_{f}^{S_{p}}\right)$ defined by $g_{v}^{0}=w_{l}$ at all $v \in S$ and $g_{v}^{0}=1$ if $v \notin S$. Then we have

$$
\widehat{\Phi}_{\left[g^{0}\right]}^{w_{2}}\left(\mathcal{E}^{\text {ord }}(\Psi \mid \mathbf{1}, \mathfrak{n})\right)=L_{p}^{S}\left(-1, \Psi_{+} \tau_{\mathcal{K} / \mathcal{F}}\right) \cdot L_{p}^{S}(\Psi, \Sigma) \cdot c(\Lambda),
$$

where $c(\Lambda)=\Psi_{\Sigma_{p}^{c}}\left(\operatorname{rec}_{\mathcal{K}}(p)\right) c_{\mathcal{K}}(p) \in \Lambda^{\times}$. Moreover, we have the following equality between ideals of $\Lambda$

$$
\left(\widehat{\Phi}_{[g]}^{w_{2}}\left(\mathcal{E}^{\text {ord }}(\Psi \mid \mathbf{1}, \mathfrak{n})\right)\right)_{g \in C(K)}=\left(L_{p}^{S}\left(-1, \Psi_{+} \tau_{\mathcal{K} / \mathcal{F}}\right) \cdot L_{p}^{S}(\Psi, \Sigma)\right) .
$$

Proof. Let $\mathcal{E}_{\chi}:=\widehat{E}^{\text {ord }}(\chi \mid \mathbf{1}, \mathfrak{n})$ for $\chi=\psi \epsilon$ with $\widehat{\epsilon} \in \mathfrak{X}_{\Lambda}^{+}$. From the discussion in $\$ 5.6 .5$ and Prop. 6.5, we deduce that

$$
\begin{aligned}
\frac{1}{\Omega_{p}^{k \Sigma}} \cdot \widehat{\Phi}_{\left[g^{0}\right]}^{w_{2}}\left(\mathcal{E}_{\chi}\right)= & \iota_{p} \iota_{\infty}^{-1}\left(\frac{(2 \pi i)^{k d}}{\Omega_{\infty}^{k \Sigma}} \cdot L_{(\infty, p)}^{S}\left(-1, \chi_{+} \tau_{\mathcal{K} / \mathcal{F}}\right) \cdot L_{(\infty, p)}^{S}(0, \chi)\right) \\
& \cdot \widehat{\chi}_{\Sigma_{p}^{c}}(p) c_{\mathcal{K}}(p),
\end{aligned}
$$

and the $\mathcal{O}_{\mathfrak{p}}$-ideal $\left(\widehat{\Phi}_{[g]}^{w_{2}}\left(\mathcal{E}_{\chi}\right)\right)_{g \in C(K)}$ is generated by $\widehat{\Phi}_{\left[g^{0}\right]}^{w_{2}}\left(\mathcal{E}_{\chi}\right)$. The proposition thus follows from the interpolation property of $\mathcal{E}^{\text {ord }}(\Psi \mid \mathbf{1}, \mathfrak{n})$.

\section{Eisenstein IDEAL AND $p$-ADiC $L$-FUnCtions}

The aim of this section is to show the non-vanishing modulo $\mathfrak{m}_{\Lambda}$ of some FourierJacobi coefficient of our $\Lambda$-adic ordinary Eisenstein series $\mathcal{E}^{\text {ord }}(\Psi \mid \mathbf{1}, \mathfrak{n})$ associated to a branch character $\psi$ and an auxiliary ideal $\mathfrak{n}$ satisfying (a1) and (a2). We will use the explicit calculation of Fourier-Jacobi coefficients of Eisenstein series on $U(2,1)$ due to Murase and Sugano [MS02]. This non-vanishing result enables us to show that the Eisenstein ideal on $U(2,1)$ indeed is divisible by a product of Deligne-Ribet $p$-adic $L$-function and Katz $p$-adic $L$-function (Cor. 7.20).

7.1. We recall some notation from $\mathrm{MS02}$, §3.5]. Let $m \in \mathcal{F}_{+}$be a totally positive element in $\mathcal{F}$ and let $\mathfrak{a}$ be a fractional ideal of $\mathcal{K}$. Let $\mathcal{D}_{\mathcal{F}}$ be the absolute difference of $\mathcal{F}$. Put

$$
\mathfrak{f}(\mathfrak{a}, m)=\mathcal{F} \cap m \mathcal{D}_{\mathcal{F}}\left(\vartheta^{-1} \mathcal{D}_{\mathcal{K} / \mathcal{F}}^{-1}\right) \mathrm{N}_{\mathcal{K} / \mathcal{F}}(\mathfrak{a})
$$


For $v \in \mathbf{h}$, set

$$
\delta_{\mathcal{K}_{v} / \mathcal{F}_{v}}=v\left(D_{\mathcal{K} / \mathcal{F}}\right) ; \mu_{v}(\mathfrak{a}, m)=v(\mathfrak{f}(\mathfrak{a}, m))
$$

For a character $\kappa_{v}$ of $\mathcal{K}_{v}^{\times}$, we let $a\left(\kappa_{v}\right)$ be the smallest non-negative integer $n$ such that $\kappa_{v}$ is trivial on $\left(1+\mathfrak{P}_{v}^{n}\right) \cap \mathcal{O}_{\mathcal{K}_{v}}^{\times}$, where

$$
\mathfrak{P}_{v}= \begin{cases}\varpi_{v} \mathcal{O}_{\mathcal{K}_{v}} & \text { if } v \text { is split, } \\ \text { the maximal ideal of } \mathcal{O}_{\mathcal{K}_{v}} & \text { if } v \text { is inert or ramified. }\end{cases}
$$

Let $\epsilon\left(s, \kappa_{v}, \boldsymbol{\psi}_{m, \mathcal{K}}\right)$ be Tate's epsilon factor, where $\boldsymbol{\psi}_{m, \mathcal{K}}:=\boldsymbol{\psi}_{m} \circ \operatorname{Tr}_{\mathcal{K} / \mathcal{F}}$ (recall that $\boldsymbol{\psi}_{m}: \mathbf{A}_{\mathcal{F}} / \mathcal{F} \rightarrow \mathbf{C}^{\times}$is the additive character $\left.x \mapsto \boldsymbol{\psi}(m x)\right)$ and let

$$
W_{m}\left(\kappa_{v}\right)=\frac{\epsilon\left(\frac{1}{2}, \kappa_{v}, \boldsymbol{\psi}_{m, \mathcal{K}}\right)}{\left|\epsilon\left(\frac{1}{2}, \kappa_{v}, \boldsymbol{\psi}_{m, \mathcal{K}}\right)\right|}
$$

be the local root number of $\kappa_{v}$. If $\kappa$ is a unitary Hecke character of $\mathcal{K}^{\times}$, we denote by $W(\kappa)$ the global root number of $\kappa$. It is well known that $W(\kappa)=\prod_{v} W_{m}\left(\kappa_{v}\right)$.

We shall consider a set of special Hecke characters of $\mathcal{K}^{\times}$. Let $\mathcal{X}$ be the set of Hecke characters of $\mathcal{K}^{\times}$such that

$$
\left.\kappa\right|_{\mathbf{A} \times}=\tau_{\mathcal{K} / \mathcal{F}} ; \kappa_{\infty}\left(z_{\infty}\right)=\prod_{\sigma \in \Sigma} \frac{\left|z_{\sigma}\right|}{z_{\sigma}} \quad\left(z_{\infty}=\left(z_{\sigma}\right) \in \mathcal{K}_{\infty}^{\times}\right) .
$$

If $\mathfrak{f}(\mathfrak{a}, m)$ is an integral ideal, we define a subset $\mathcal{X}_{0}(\mathfrak{a}, m)$ of $\mathcal{X}$ which consists of characters $\kappa \in \mathcal{X}$ such that

- $W_{m}\left(\kappa_{v}\right)=\kappa_{v}(\vartheta)$ for all $v \in \mathbf{h}$,

$$
a\left(\kappa_{v}\right)= \begin{cases}\mu_{v}(\mathfrak{a}, m) & \text { if } v \text { is unramified in } \mathcal{K} \\ 2\left(\mu_{v}(\mathfrak{a}, m)+\delta_{\mathcal{K}_{v} / \mathcal{F}_{v}}\right) & \text { if } \delta_{\mathcal{K}_{v} / \mathcal{F}_{v}}>0 \text { and } \mu_{v}(\mathfrak{a}, m)>0 \\ 2 \delta_{\mathcal{K}_{v} / \mathcal{F}_{v}} \text { or } 2 \delta_{\mathcal{K}_{v} / \mathcal{F}_{v}}-1 & \text { if } \delta_{\mathcal{K}_{v} / \mathcal{F}_{v}}>0 \text { and } \mu_{v}(\mathfrak{a}, m)=0\end{cases}
$$

(cf. the definition of $\mathcal{X}_{0, \text { prim }}^{+}(\mathfrak{a}, m)$ in [MS02, (3.10), page 380]). Note that the global root number $W(\kappa)$ of $\kappa$ is +1 for $\kappa \in \mathcal{X}_{0}(\mathfrak{a}, m)$.

Remark 7.1. Let $\ell>2$ be a rational prime which is split completely in $\mathcal{K}$ and let $\kappa \in \mathcal{X}_{0}(\mathfrak{a}, m)$. Suppose that $\ell$ is prime to the conductor of $\kappa$, the places in $S$ and $\vartheta$. Let $\mathfrak{L}$ be a prime of $\mathcal{K}$ lying above $\ell$ and let $\mathfrak{l}=\mathfrak{L} \cap \mathcal{F}$. Let $\mathcal{K}_{\mathfrak{l}_{\infty}}^{-}$be the maximal $\mathfrak{l}$-ramified anticyclotomic pro- $\ell$ extension of $\mathcal{K}$ and let $\mathfrak{X}_{\mathfrak{l}}^{-}$be the set of characters of $\operatorname{Gal}\left(\mathcal{K}_{\mathfrak{l} \infty}^{-} / \mathcal{K}\right)$ of finite order. Note that if $\nu \in \mathfrak{X}_{\mathfrak{l}}^{-}$has conductor $\mathfrak{l}^{a(\nu)}$, then $W_{m}(\kappa \nu)=W_{m}(\kappa)$ and $\kappa \nu \in \mathcal{X}_{0}\left(\mathfrak{a} \mathfrak{L}^{a(\nu)}, m\right)$.

\subsection{Theta functions with complex multiplication.}

7.2.1. Adelic theta functions. We identify $\underline{\mathcal{K}}^{1}=U(W)$ with a subgroup of $U(V)$ via $t \mapsto \operatorname{diag}(1, t, 1)$. Define the algebraic subgroup $R$ of $U(V)$ by

$$
R=\left\{t n \mid n \in N, t \in \underline{\mathcal{K}}^{1}\right\} .
$$

Define the function $\mathbf{e}_{m}: \mathbf{C}^{\Sigma} \rightarrow \mathbf{C}^{\times}$by

$$
\mathbf{e}_{m}(w)=\exp \left(2 \pi i \sum_{\sigma \in \Sigma} \sigma(m) w_{\sigma}\right) \quad\left(w=\left(w_{\sigma}\right)_{\sigma \in \Sigma}\right) .
$$


Let $\mathbf{T}_{\text {hol }}^{m}$ be the space of smooth functions $\Theta: R(\mathcal{F}) \backslash R(\mathbf{A}) \rightarrow \mathbf{C}$ which satisfy the following properties:

- $\Theta\left(n(0, x) r t_{\infty}\right)=\boldsymbol{\psi}_{m}(x) \Theta(r)$ for $x \in \mathbf{A}, r \in R(\mathbf{A})$ and $t_{\infty} \in \mathcal{K}_{\infty}^{1}$.

- For every $r_{f} \in R\left(\mathbf{A}_{f}\right)$, the function $w_{\infty} \mapsto \mathbf{e}_{m}\left(-\frac{1}{2 \vartheta} w_{\infty} \overline{w_{\infty}}\right) \Theta\left(n\left(w_{\infty}, 0\right) r_{f}\right)$ is holomorphic on $\mathcal{K}_{\infty}$.

We call $\mathbf{T}_{h o l}^{m}$ the holomorphic theta functions of index $m$. We equip $\mathbf{T}_{h o l}^{m}$ with the inner product defined by

$$
\left(\Theta, \Theta^{\prime}\right)=\int_{R(\mathcal{F}) \backslash R(\mathbf{A})} \Theta(r) \overline{\Theta^{\prime}(r)} d r \quad\left(\Theta, \Theta^{\prime} \in \mathbf{T}_{h o l}^{m}\right) .
$$

We let $\mathcal{K}_{\mathbf{A}}^{1}=\underline{\mathcal{K}}^{1}(\mathbf{A})$ and $\widehat{\mathcal{K}}^{1}=\underline{\mathcal{K}}^{1}\left(\mathbf{A}_{f}\right)$ be the groups of norm one for brevity. For $t \in \mathcal{K}_{\mathbf{A}}^{1}$ and $\Theta \in \mathbf{T}_{h o l}^{m}$, denote by $\rho^{\prime}(t) \Theta(r):=\Theta(r t)$ the right translation. For an ideal $\mathfrak{a}$ and a finite place $v$, we put

$$
N\left(\mathfrak{a}_{v}\right)=\left\{n(w, x) \in N_{P}\left(\mathcal{F}_{v}\right) \mid w \in \mathfrak{a}, x+\frac{1}{2} w \vartheta^{-1} \bar{w} \in \vartheta^{-1} \mathcal{D}_{\mathcal{K} / \mathcal{F}}^{-1} \mathrm{~N}_{\mathcal{K} / \mathcal{F}}\left(\mathfrak{a}_{v}\right)\right\}
$$

and $N(\mathfrak{a})=\prod_{v \in \mathbf{h}} N\left(\mathfrak{a}_{v}\right)$. Let $\widehat{\mathcal{O}}_{\mathcal{K}}^{1}=\widehat{\mathcal{O}}_{\mathcal{K}}^{\times} \cap \mathcal{K}_{\mathbf{A}}^{1}$ be units group of norm one. Define the space $\mathbf{T}_{h o l}^{m}(\mathfrak{a})$ of holomorphic theta functions of index $m$ and level $\mathfrak{a}$ by

$$
\mathbf{T}_{h o l}^{m}(\mathfrak{a}):=\left\{\Theta \in \mathbf{T}_{h o l}^{m} \mid \Theta(r n t)=\Theta(r), n t \in N(\mathfrak{a}) \widehat{\mathcal{O}}_{\mathcal{K}}^{1}\right\} .
$$

([MS02, $\S 1.13$ and (1.14)]). By definition, $\mathbf{T}_{h o l}^{m}(\mathfrak{a})=\{0\}$ if $\mathfrak{f}(\mathfrak{a}, m)$ is not integral. Henceforth, we assume

$$
\mathfrak{f}(\mathfrak{a}, m) \text { is an integral ideal }
$$

until the end of this section.

Define the endomorphism $\mathcal{P}_{\mathfrak{a}}$ of $\mathbf{T}_{h o l}^{m}$ by

$$
\mathcal{P}_{\mathfrak{a}} \Theta(r)=\int_{N(\mathfrak{a})} \Theta(r n) d_{\mathfrak{a}} n
$$

where $d_{\mathfrak{a}} n$ is the Haar measure on $N(\mathfrak{a})$ normalized so that $\operatorname{vol}\left(N(\mathfrak{a}), d_{\mathfrak{a}} n\right)=1$ ([MS02, §3.1 (3.1)]). Then $\mathcal{P}_{\mathfrak{a}}$ is a self-adjoint projector ( $c f$. [MS00, Lemma 5.4]), and

$$
\mathbf{T}_{\text {hol }}^{m}(\mathfrak{a})=\left\{\Theta \in \mathcal{P}_{\mathfrak{a}}\left(\mathbf{T}_{\text {hol }}^{m}\right) \mid \rho^{\prime}(t) \Theta=\Theta, t \in \widehat{\mathcal{O}}_{\mathcal{K}}^{1}\right\} .
$$

The space of primitive theta functions $\mathbf{T}_{\text {hol,prim }}^{m}(\mathfrak{a})$ is defined by

$$
\mathbf{T}_{\text {hol,prim }}^{m}(\mathfrak{a})=\left\{\Theta \in \mathbf{T}_{\text {hol }}^{m}(\mathfrak{a}) \mid \mathcal{P}_{\mathfrak{b}} \Theta=0 \text { for all } \mathfrak{b} \supset \mathfrak{a}, \mathfrak{b} \neq \mathfrak{a}\right\} .
$$

Let $\left(\rho, V^{m}\right)$ be the smooth lattice model of the Heisenberg group $N(\mathbf{A})$ with central character $\boldsymbol{\psi}_{m}$ defined in [MS02, $\left.\S 2.7\right]$. For $\kappa \in \mathcal{X}_{0}(\mathfrak{a}, m)$, let $\mathcal{M}_{\kappa}$ be the Weil presentation of $\mathcal{K}_{\mathbf{A}}^{1}$ on $V^{m}$ defined in [MS02, page 365]. We note that $\mathcal{M}_{\kappa}(\bar{z} / z)=$ $\kappa^{-1}(z) \mathcal{M}(z)$ for $z \in \mathbf{A}_{\mathcal{K}}^{\times}$, where $\mathcal{M}$ is the universal Weil representation 3 defined in [MS00, §4.3]. Then $\mathcal{M}_{\kappa}$ can be extended to $R(\mathbf{A})$ by setting $\mathcal{M}_{\kappa}(n t)=$ $\rho(n) \mathcal{M}_{\kappa}(t), n \in N(\mathbf{A}), t \in \mathcal{K}_{\mathbf{A}}^{1}$. Let $V_{h o l}^{m} \subset \mathcal{S}\left(\mathbf{A}_{\mathcal{K}}\right)$ be the subspace of $V^{m}$ defined in [MS02, §2.25]. Define the intertwining operator $\mathcal{T}_{\kappa}^{m}: V_{h o l}^{m} \rightarrow \mathbf{T}_{h o l}^{m}$ by

$$
\mathcal{T}_{\kappa}^{m}(\Phi)(r):=\sum_{\alpha \in \mathcal{K}} \mathcal{M}_{\kappa}(r) \Phi(\alpha)
$$

\footnotetext{
${ }^{3}$ The definition of $\mathcal{M}$ does not depend on a particular choice of models of the Heisenberg group representation.
} 
We put

$$
\mathbf{T}_{\text {hol,prim }}^{m}(\mathfrak{a}, \kappa)=\left\{\Theta \in \mathbf{T}_{\text {hol,prim }}^{m}(\mathfrak{a}) \mid \Theta=\mathcal{T}_{\kappa}^{m}(\Phi) \text { for some } \Phi \in V_{\text {hol }}^{m}\right\} .
$$

Then $\mathbf{T}_{\text {hol,prim }}^{m}(\mathfrak{a}, \kappa)$ is the space of primitive theta functions attached to $(\mathfrak{a}, \kappa)$ defined in [MS02, (3.3), page 377]. The following multiplicity one theorem for primitive theta functions is proved in [MS02, Thm. 3.8].

Theorem 7.2. For each $\kappa \in \mathcal{X}_{0}(\mathfrak{a}, m), \operatorname{dim}_{\mathbf{C}} \mathbf{T}_{h o l, p r i m}^{m}(\mathfrak{a}, \kappa)=1$.

7.2.2. Shintani operators. We recall that the unitary (adelic) Shintani operator $\mathscr{F}^{*}(z) \in \operatorname{End}\left(\mathbf{T}_{\text {hol,prim }}^{m}(\mathfrak{a})\right)$ for $z \in \mathcal{K}_{v}^{\times}$and a finite place $v \nmid \mathfrak{f}(\mathfrak{a}, m) D_{\mathcal{K} / \mathcal{F}}$ is defined by

$$
\mathscr{F}^{*}(z) \Theta:= \begin{cases}|1-\bar{z} / z|_{\mathcal{K}_{v}}^{\frac{1}{2}} \mathcal{P}_{\mathfrak{a}} \rho^{\prime}(z / \bar{z}) \Theta & \text { if } z / \bar{z} \notin \mathcal{O}_{\mathcal{K}_{v}}^{\times}, \\ \tau_{\mathcal{K}_{v} / \mathcal{F}_{v}}(x) \cdot \Theta & \text { if } z=x u, x \in \mathcal{F}_{v}^{\times}, u \in \mathcal{O}_{\mathcal{K}_{v}}^{\times} .\end{cases}
$$

Then $\mathscr{F}^{*}(z)=L^{*}(z)|z|_{\mathcal{K}_{v}}^{\frac{1}{2}}$, where $L^{*}(z)$ is the classical Shintani operator introduced in [Fin06, page 775]. The following proposition is proved in [Fin06, Prop. 2.1] with a key ingredient [MS00, Prop. 7.3].

Proposition 7.3. The Shintani operator $\mathscr{F}^{*}$ can be extended to a unique morphism $\mathscr{F}^{*}: \mathcal{K}^{\times} \backslash \mathbf{A}_{\mathcal{K}}^{\times} \rightarrow \operatorname{End}\left(\mathbf{T}_{\text {hol,prim }}^{m}(\mathfrak{a})\right)$ such that $\left.\mathscr{F}^{*}\right|_{\mathbf{A}^{\times}}=\tau_{\mathcal{K} / \mathcal{F}}$ and $\left.\mathscr{F}^{*}\right|_{\mathcal{K}_{\infty}^{\times} \widehat{\mathcal{O}}_{\mathcal{K}}^{\times}}=\mathcal{M}$. Furthermore, eigenfunctions of $\mathscr{F}^{*}$ in $\mathbf{T}_{\text {hol,prim }}^{m}(\mathfrak{a})$ with the eigencharacter $\kappa$ are primitive theta functions attached to $(\mathfrak{a}, \kappa)$.

We define the projector $\mathcal{P}_{\kappa}: \mathbf{T}_{\text {hol,prim }}^{m}(\mathfrak{a}) \rightarrow \mathbf{T}_{\text {hol,prim }}^{m}(\mathfrak{a}, \kappa)$ by

$$
\mathcal{P}_{\kappa} \Theta=\int_{C_{\mathcal{K}}^{-}} \kappa^{-1}(z) \mathscr{F}^{*}(z) \Theta d^{\times} z, C_{\mathcal{K}}^{-}:=\mathcal{K}^{\times} \mathbf{A}_{f}^{\times} \backslash \mathbf{A}_{\mathcal{K}, f}^{\times},
$$

where the Haar measure $d^{\times} z$ is normalized so that $\operatorname{vol}\left(C_{\mathcal{K}}^{-}, d^{\times} z\right)=1$. Note that $\mathcal{P}_{\kappa}$ is self-adjoint since $\mathscr{F}^{*}$ and $\kappa$ are unitary. Let $\mathfrak{f}_{\mathfrak{a}}:=\mathfrak{f}(\mathfrak{a}, m) D_{\mathcal{K} / \mathcal{F}}$. By MS00, Lemma 7.4], we have $\left.\mathscr{F}^{*}\right|_{\mathcal{U}\left(\mathfrak{f}_{\mathfrak{a}}\right)}=1$. Fixing a system of representatives $\mathcal{C}^{-}$for the relative ray class group $C_{\mathcal{K}}^{-}\left(\mathfrak{f}_{\mathfrak{a}}\right):=C_{\mathcal{K}}^{-} / \mathcal{U}\left(\mathfrak{f}_{\mathfrak{a}}\right)$ modulo $\mathfrak{f}_{\mathfrak{a}}$ in $\left(\mathbf{A}_{\mathcal{K}, f}^{\left(p \mathfrak{f}_{\mathfrak{a}}\right)}\right)^{\times}$, we can write

$$
\mathcal{P}_{\kappa} \Theta=\frac{1}{\sharp\left(C_{\mathcal{K}}^{-}\left(\mathfrak{f}_{\mathfrak{a}}\right)\right)} \cdot \sum_{z \in \mathcal{C}^{-}} \kappa^{-1}(z) \mathscr{F}^{*}(z) \Theta \quad\left(\Theta \in \mathbf{T}_{\text {hol,prim }}^{m}(\mathfrak{a}), \kappa \in \mathcal{X}_{0}(\mathfrak{a}, m)\right) .
$$

On the other hand, for each finite place $v$, let $\mathcal{P}_{\mathfrak{a}, \text { prim,v } v}$ be the self-adjoint projector onto the space of local primitive theta functions introduced in [MS00, §5.7]. Define the a-primitive projector $\mathcal{P}_{\mathfrak{a}, \text { prim }}=\prod_{v} \mathcal{P}_{\mathfrak{a}_{v}, \text { prim }, v}: \mathbf{T}_{\text {hol }}^{m} \rightarrow \mathbf{T}_{\text {hol,prim }}^{m}(\mathfrak{a})$. Finally, we define the self-adjoint projector

$$
\mathcal{P}_{\mathfrak{a}, \kappa}:=\mathcal{P}_{\kappa} \circ \mathcal{P}_{\text {prim, }, \mathfrak{a}}: \mathbf{T}_{\text {hol }}^{m} \rightarrow \mathbf{T}_{\text {hol,prim }}^{m}(\mathfrak{a}, \kappa) .
$$

7.2.3. Classical theta functions and their integral structures. We review the analytic theory of theta functions. A standard reference is [Mum70, Chapter I] (see also [Fin06, §2]). Define a positive-definite Hermitian form $H^{m}$ on $\mathbf{C}^{\Sigma}$ by

$$
H^{m}\left(w, w^{\prime}\right)=(-2 i) \sum_{\sigma \in \Sigma} w_{\sigma} \sigma\left(m \vartheta^{-1}\right) \overline{w_{\sigma}^{\prime}} \quad\left(w=\left(w_{\sigma}\right)_{\sigma \in \Sigma}, w^{\prime}=\left(w_{\sigma}^{\prime}\right)_{\sigma \in \Sigma}\right) .
$$

Define a cocycle $e: \mathbf{C}^{\Sigma} \times \mathcal{K} \rightarrow \mathbf{C}^{\times}$by

$$
e(w, l):=\exp \left(\pi H^{m}\left(w+\frac{l}{2}, l\right)\right) .
$$


Here $\mathcal{K}$ is embedded in $\mathbf{C}^{\Sigma}$ by $l \mapsto(\sigma(l))_{\sigma \in \Sigma}$. Choose a finite idele $u=\left(u_{v}\right) \in$ $\mathbf{A}_{\mathcal{K}, f}$ such that $\mathcal{O}_{\mathcal{K}_{v}}=\mathcal{O}_{\mathcal{F}_{v}} \oplus \mathcal{O}_{\mathcal{F}_{v}} u_{v}$ for each $v \in \mathbf{h}$. For $w \in \mathbf{A}_{\mathcal{K}, f}$, let $x_{w}, y_{w}$ be the unique elements in $\mathbf{A}_{f}$ such that $\frac{1}{2} w \bar{w} \vartheta^{-1}=x_{w}+y_{w} u$. For $l \in \mathcal{K}$, put $\alpha_{u}(l)=\boldsymbol{\psi}_{m}\left(-x_{l}\right)$.

Let $\mathfrak{b}$ be a prime-to- $p$ ideal of $\mathcal{K}$ such that $\mathfrak{f}(\mathfrak{b}, m)$ is an integral ideal. Thus $\alpha_{\mathfrak{b}}:=\left.\alpha_{u}\right|_{\mathfrak{b}}$ is a semi-character on $\mathfrak{b}$ which takes value \pm 1 and is independent of the choice of $u$. It is straightforward to verify the following equation:

$$
\alpha_{\mathfrak{b}}\left(l_{1}\right) \alpha_{\mathfrak{b}}\left(l_{2}\right) e\left(w+l_{2}, l_{1}\right) e\left(w, l_{2}\right)=\alpha_{\mathfrak{b}}\left(l_{1}+l_{2}\right) e\left(w, l_{1}+1_{2}\right), l_{1}, l_{2} \in \mathfrak{b} .
$$

We define a line bundle $L_{\mathfrak{b}}^{m}$ on $\mathbf{C}^{\Sigma} / \mathfrak{b}$ by $\mathbf{C}^{\Sigma} \times \mathbf{C} / \mathfrak{b}$ with the action of $l \in \mathfrak{b}$ given by

$$
l(w, x)=\left(w+l, \alpha_{\mathfrak{b}}(l) e(w, l) x\right) .
$$

The canonical rigidification of $L_{\mathfrak{b}}^{m}$ along the zero section is given by $\epsilon_{L}: \mathbf{C} \stackrel{\sim}{\rightarrow}$ $L_{\mathfrak{b}}^{m}(0), x \mapsto[(0, x)]$. The space of global sections $\Gamma\left(\mathbf{C}^{\Sigma} / \mathfrak{b}, L_{\mathfrak{b}}^{m}\right)$ consists of all holomorphic functions $\boldsymbol{\theta}$ on $\mathbf{C}^{\Sigma}$ satisfying the equation

$$
\boldsymbol{\theta}(w+l)=\alpha_{\mathfrak{b}}(l) e(w, l) \boldsymbol{\theta}(w), l \in \mathfrak{b} .
$$

The line bundle $L_{\mathfrak{b}}^{m}$ is ample and $\operatorname{dim}_{\mathbf{C}} \Gamma\left(\mathbf{C}^{\Sigma} / \mathfrak{b}, L_{\mathfrak{b}}^{m}\right)=\mathrm{N}_{\mathcal{F} / \mathbf{Q}}(\mathfrak{f}(\mathfrak{b}, m)) D_{\mathcal{K}}$. For $l \in \mathcal{K}$ and a complex-valued function $f$ on $\mathbf{C}^{\Sigma}$, define

$$
A_{l}(f)(w):=e(w, l)^{-1} f(w+l) .
$$

The operators $A_{l}$ satisfy the commutation relation

$$
A_{l_{1}} A_{l_{2}}=\mathbf{e}_{m}\left(\frac{1}{2 \vartheta}\left(l_{1} \bar{l}_{2}-\bar{l}_{1} l_{2}\right)\right) A_{l_{1}+l_{2}} .
$$

If $l \in \mathfrak{b}^{\vee}:=\mathfrak{f}(\mathfrak{b}, m)^{-1} D_{\mathcal{K} / \mathcal{F}}^{-1} \mathfrak{b}$, then $A_{l}$ is an endomorphism of $\Gamma\left(\mathbf{C}^{\Sigma} / \mathfrak{b}, L_{\mathfrak{b}}^{m}\right)$ and acts by multiplication by $\alpha_{\mathfrak{b}}(l)$ if $l \in \mathfrak{b}$. In addition, $\left\{A_{l}\right\}_{l \in \mathfrak{b} \vee}$ are elements in Mumford's theta group $\mathscr{G}\left(L\left(H^{m}, \alpha_{\mathfrak{b}}\right)\right)$ Mum70, page 235]. For $\Theta \in \mathbf{T}_{\text {hol }}^{m}(\mathfrak{b})$ and $\mathbf{c} \in \widehat{\mathcal{K}}^{1}$, we define a classical theta function $\boldsymbol{\theta}_{\mathbf{c}} \in \Gamma\left(\mathbf{C}^{\Sigma} / \mathfrak{b} \mathbf{c}, L_{\mathfrak{b c}}^{m}\right)$ by

$$
\boldsymbol{\theta}_{\mathbf{c}}(w):=\mathbf{e}_{m}\left(-\frac{1}{2 \vartheta} w \bar{w}\right) \Theta(n(w, 0) \mathbf{c}) .
$$

Fixing a set of representatives $I_{\mathcal{K}}^{1}$ of the ideal class group $\mathcal{K}^{1} \backslash \mathcal{K}_{\mathbf{A}}^{1} / \widehat{\mathcal{O}}_{\mathcal{K}}^{1}$ in $\mathcal{K}^{1}\left(\mathbf{A}_{f}^{(p)}\right)$, the map $\Theta \mapsto\left(\boldsymbol{\theta}_{\mathbf{c}}\right)_{\mathbf{c}}$ gives rise to an identification $\mathbf{T}_{h o l}^{m}(\mathfrak{b})=\prod_{\mathbf{c} \in I_{\mathcal{K}}^{1}} \Gamma\left(\mathbf{C}^{\Sigma} / \mathfrak{b} \mathbf{c}, L_{\mathfrak{b c}}^{m}\right)$.

We discuss briefly the integral structures of theta functions via their geometric interpretation. A more detailed account can be found in [Fin06, §3] and the references therein. By the standard theory of abelian varieties with complex multiplication, the pair $\left(\mathbf{C}^{\Sigma} / \mathfrak{b}, L_{\mathfrak{b}}^{m}\right)$ descends to $\left(\mathcal{B}_{\mathfrak{b}}, \mathcal{L}_{\mathfrak{b}}^{m}\right)$ over a discrete valuation ring $R_{\mathcal{N}}:=\overline{\mathbf{Z}}_{p} \cap \mathcal{N}$ for some number field $\mathcal{N}$ so that $\left(\mathcal{B}_{\mathfrak{b}}, \mathcal{L}_{\mathfrak{b}}^{m}\right) \otimes_{R_{\mathcal{N}}} \mathbf{C} \simeq\left(\mathbf{C}^{\Sigma} / \mathfrak{b}, L_{\mathfrak{b}}^{m}\right)$. Here we are making use of the fixed isomorphism $\mathbf{C} \simeq \mathbf{C}_{p}$. We fix a rigidification $\epsilon_{\mathcal{L}}: \mathbf{A}_{/ R_{\mathcal{N}}}^{1} \stackrel{\sim}{\rightarrow} \mathcal{L}_{\mathfrak{b} / R_{\mathcal{N}}}^{m}$ so that $\epsilon_{L}^{-1} \circ \epsilon_{\mathcal{L}}(x)=x$ for every $x \in \mathbf{C}=\mathbf{A}^{1}(\mathbf{C})$. Define the $\overline{\mathbf{Z}}_{p}$-submodule $\mathbf{T}_{\text {int }}^{m}(\mathfrak{b})$ of $\mathbf{T}_{\text {hol }}^{m}(\mathfrak{b})$ by

$$
\mathbf{T}_{i n t}^{m}(\mathfrak{b}):=\prod_{\mathbf{c} \in I_{\mathcal{K}}^{1}} \Gamma\left(\mathcal{B}_{\mathfrak{b c}}, \mathcal{L}_{\mathfrak{b c}}^{m}\right) \otimes_{R_{\mathcal{N}}} \overline{\mathbf{Z}}_{p}
$$

We call $\mathbf{T}_{\text {int }}^{m}(\mathfrak{b})$ the space of $p$-integral theta functions. By the ampleness of the line bundles $\mathcal{L}_{\mathfrak{b c}}^{m}$, we have $\mathbf{T}_{\text {int }}^{m}(\mathfrak{b}) \otimes_{\overline{\mathbf{z}}_{p}} \mathbf{C}=\mathbf{T}_{\text {hol }}^{m}(\mathfrak{b})$. The complex theta group $\mathscr{G}\left(L\left(H^{m}, \alpha_{\mathfrak{b}}\right)\right)$ is the set of $\mathbf{C}$-points of the theta group scheme $\mathscr{G}\left(\mathcal{L}_{\mathfrak{b}}^{m}\right) / R_{\mathcal{N}}$ Mum70, 
page 221]. The operators $\left\{A_{l}\right\}_{l \in \mathfrak{b} \vee}$ are torsion points in $\mathscr{G}\left(L_{\mathfrak{b}}^{m}\right)(\mathbf{C})$ in view of (7.1). In particular, this implies that $\left\{A_{l}\right\}_{l \in \mathfrak{b} \vee}$ belong to $\mathscr{G}\left(\mathcal{L}_{\mathfrak{b}}^{m}\right)(\overline{\mathbf{Z}})$ and preserve $\mathbf{T}_{\text {int }}^{m}(\mathfrak{b})$.

7.2.4. Integral projectors onto primitive theta functions. Let $A_{1}$ (resp. $A_{2}$ ) be the set of unramified (resp. split) places of $\mathcal{F}$ where $\kappa$ is ramified. Let $h_{\mathcal{K}}^{-}=h_{\mathcal{K}} / h_{\mathcal{F}}$ be the relative class number of $\mathcal{K}$. Put

$$
\begin{aligned}
& B_{1}(\kappa)=\prod_{v \in A_{1}}\left(1+q_{v}^{-1}\right), \quad B_{2}(\kappa)=\prod_{v \in A_{2}}\left(1-q_{v}^{-1}\right), \\
& B^{*}(\kappa)=h_{\mathcal{K}}^{-} \cdot\left[\mathcal{O}_{\mathcal{K}}^{\times}: \mathcal{O}_{\mathcal{F}}^{\times}\right]^{-1} B_{1}(\kappa) B_{2}(\kappa) .
\end{aligned}
$$

Here $q_{v}$ is the cardinality of the residue field of $\mathcal{O}_{\mathcal{F}_{v}}$. Suppose that $\mathfrak{b} \subset \mathfrak{a}$. We note that $\mathfrak{a} \subset \mathfrak{b}^{\vee}$ as $\mathfrak{f}(\mathfrak{a}, m)$ is integral. From the equation $\mathcal{P}_{\mathfrak{a}} \Theta=\left(\sum_{l \in \mathfrak{a} / \mathfrak{b}} \alpha_{\mathfrak{a}}(l) A_{l} \boldsymbol{\theta}_{\mathbf{c}}\right)_{\mathbf{c}}$ for $\Theta=\left(\boldsymbol{\theta}_{\mathbf{c}}\right)_{\mathbf{c}} \in \mathbf{T}_{\text {hol }}^{m}(\mathfrak{b})$, it follows immediately that the operator $\mathcal{P}_{\mathfrak{a}}$ is indeed a $p$-integral self-adjoint projector preserving $\mathbf{T}_{i n t}^{m}(\mathfrak{b})$ and $\mathcal{P}_{\mathfrak{a}}\left(\mathbf{T}_{i n t}^{m}(\mathfrak{b})\right) \subset \mathbf{T}_{i n t}^{m}(\mathfrak{a})$. By [MS00, Lemma 5.9], the normalized primitive projector $\mathcal{P}_{\mathfrak{a}, \text { prim }}^{*}:=B_{2}(\kappa) \mathcal{P}_{\mathfrak{a}, \text { prim }}$ is a $\mathbf{Z}_{(p)}$-linear combination of $\left\{\mathcal{P}_{\mathfrak{a}^{\prime}}\right\}_{\mathfrak{a}^{\prime}}$ for prime-to- $p$ ideals $\mathfrak{a}^{\prime} \supset \mathfrak{a}$, so we find that $\mathcal{P}_{\mathfrak{a}, \text { prim }}^{*}$ is also a $p$-integral self-adjoint projector.

On the other hand, we claim that $B^{*}(\kappa) / \sharp\left(C_{\mathcal{K}}^{-}\left(\mathfrak{f}_{\mathfrak{a}}\right)\right) \in \mathbf{Z}_{(p)}$. Indeed, by definition $C_{\mathcal{K}}^{-}\left(\mathfrak{f}_{\mathfrak{a}}\right)=\mathcal{K}^{\times} \mathbf{A}_{f}^{\times} \backslash \mathbf{A}_{\mathcal{K}, f}^{\times} / \mathcal{U}\left(\mathfrak{f}_{\mathfrak{a}}\right)$, so we find that

$$
\sharp\left(C_{\mathcal{K}}^{-}\left(\mathfrak{f}_{\mathfrak{a}}\right)\right) \mid h_{\mathcal{K}}^{-} \cdot \prod_{v \mid \mathfrak{f}_{\mathfrak{a}}} \sharp\left(\mathcal{O}_{\mathcal{K}_{v}}^{\times} /\left(1+\mathfrak{f}_{\mathfrak{a}} \mathcal{O}_{\mathcal{K}_{v}}\right) \mathcal{O}_{\mathcal{F}_{v}}^{\times}\right) .
$$

Since $p>2$ is unramified in $\mathcal{K}$ and the conductor $\mathfrak{c}(\kappa)$ of $\kappa$ and $\mathfrak{f}_{\mathfrak{a}}$ share the same support $\left(\kappa \in \mathcal{X}_{0}(\mathfrak{a}, m)\right)$,

$$
p \text {-part of } \sharp\left(C_{\mathcal{K}}^{-}\left(\mathfrak{f}_{\mathfrak{a}}\right)\right) \mid p \text {-part of } h_{\mathcal{K}}^{-} \cdot \prod_{v \in A_{1}}\left(1-\tau_{\mathcal{K} / \mathcal{F}}\left(\varpi_{v}\right) q_{v}\right) \mid p \text {-part of } B^{*}(\kappa) \text {. }
$$

This proves the claim. It follows that the operator $\mathcal{P}_{\kappa}^{*}:=B^{*}(\kappa) \cdot \mathcal{P}_{\kappa}$ is a $\mathbf{Z}_{(p)}$-linear combination of $p$-integral Shintani operators $\left\{\mathscr{F}^{*}(z)\right\}_{z \in \mathcal{C}^{-}}$by the choice of $\mathcal{C}^{-}$. In particular, $\mathcal{P}_{\kappa}^{*}$ also preserves the space $\mathbf{T}_{i n t}^{m}(\mathfrak{a})$.

We have thus constructed a $p$-integral self-adjoint operator

$$
\mathcal{P}_{\mathfrak{a}, \kappa}^{*}:=\mathcal{P}_{\kappa}^{*} \circ \mathcal{P}_{\mathfrak{a}, \text { prim }}^{*}=B^{*}(\kappa) B_{2}(\kappa) \cdot \mathcal{P}_{\mathfrak{a}, \kappa}
$$

such that $\mathcal{P}_{\mathfrak{a}, \kappa}^{*} \mathbf{T}_{\text {int }}^{m}(\mathfrak{a}) \subset \mathbf{T}_{\text {hol,prim }}^{m}(\mathfrak{a}, \kappa) \cap \mathbf{T}_{\text {int }}^{m}(\mathfrak{a})$, and $\mathcal{P}_{\mathfrak{a}, \kappa}^{*}$ acts $\mathbf{T}_{\text {hol,prim }}^{m}(\mathfrak{a}, \kappa)$ via the multiplication map by $B^{*}(\kappa) B_{2}(\kappa) \in \mathbf{Z}_{(p)}$.

7.3. Fourier-Jacobi coefficients. Let $a=\left(a_{v}\right) \in\left(\mathbf{A}_{\mathcal{K}, f}^{(p)}\right)^{\times}$such that $\mathfrak{a}=a \mathcal{O}_{\mathcal{K}, f} \cap$ $\mathcal{K}$ is a prime-to- $p$ fractional ideal. Let $F \in \mathbf{M}_{k I^{-}}(K, \mathbf{C})$ be a modular form of weight $k I^{-}=(0,0 ; k \Sigma)$. Put $F_{a}(g):=F(g \mathbf{d}(a))$, where $\mathbf{d}(a)=\operatorname{diag}\left(\bar{a}, 1, a^{-1}\right)$. Define a function $F_{a}^{m}$ on $R(\mathbf{A})$ by

$$
F_{a}^{m}(r)=\int_{\mathbf{A} / \mathcal{F}} \boldsymbol{\psi}_{m}(-x) F_{a}(n(0, x) r) d x, r \in R\left(\mathbf{A}_{\mathcal{F}}\right) .
$$

Then $F_{a}^{m}$ is a holomorphic theta function of index $m$ and is called the $m$-th FourierJacobi coefficient of $F_{a}$. For $\kappa \in \mathcal{X}_{0}(\mathfrak{a}, m)$ and a non-zero $\Theta \in \mathbf{T}_{\text {hol,prim }}^{m}(\mathfrak{a}, \kappa)$, define the period integral $I(\Theta)$ by

$$
I(\Theta)=\int_{\mathcal{K}^{1} \backslash \mathcal{K}_{\mathbf{A}}^{1}} \Theta(t) d^{\times} t
$$

where $d^{\times} t$ is normalized so that $\operatorname{vol}\left(\mathcal{K}^{1} \backslash \mathcal{K}_{\mathbf{A}}^{1}, d^{\times} t\right)=1$. 
Define a linear functional $l_{\mathfrak{a}, m, \kappa}: \mathbf{M}_{k I^{-}}(K, \mathbf{C}) \rightarrow \mathbf{C}_{p}$ by

$$
l_{\mathfrak{a}, m, \kappa}(F)=\iota\left(B^{*}(\kappa) B_{2}(\kappa) \mathbf{e}_{m}\left(-i_{\Sigma}\right) \cdot \frac{\left(F_{a}^{m}, \Theta\right)}{(\Theta, \Theta)} \cdot I(\Theta) \cdot h_{\mathcal{K}}^{-}\right) .
$$

Note that $l_{\mathfrak{a}, m, \kappa}(F)$ does not depend on the choice of $\Theta$ in view of the multiplicity one theorem for primitive theta functions (Theorem 7.2). Now we examine the $p$-integrality of the linear functional $l_{\mathfrak{a}, m, \kappa}$. Suppose $F$ is a $p$-integral modular form in $\mathbf{M}_{k I^{-}}\left(K, \iota^{-1}\left(\overline{\mathbf{Z}}_{p}\right)\right)$. The Fourier-Jacobi expansion of $F_{a}$ is given by

$$
F_{a}=F_{a}^{0}+\sum_{m \in \mathcal{F}_{+}} \widetilde{F}_{a}^{m} \cdot q^{m}
$$

where $\widetilde{F}_{a}^{m}:=F_{a}^{m} \cdot \mathbf{e}_{m}\left(-i_{\Sigma}\right)$ is a $p$-integral theta function in $\mathbf{T}_{i n t}^{m}(\mathfrak{a n})$. Let $\Theta_{\mathfrak{a}, \kappa}^{m}=$ $\left(\boldsymbol{\theta}_{\mathfrak{a}, \kappa, \mathbf{c}}\right)_{\mathbf{c} \in I_{\mathcal{K}}^{1}}$ be an element in $\mathbf{T}_{\text {hol, prim }}^{m}(\mathfrak{a}, \kappa) \cap \mathbf{T}_{\text {int }}^{m}(\mathfrak{a})$ such that $\boldsymbol{\theta}_{\mathfrak{a}, \kappa, \mathbf{c}} \not \equiv 0(\bmod \mathfrak{m})$ for some c. Then we can write $\mathcal{P}_{\mathfrak{a}, \kappa}^{*} \widetilde{F}_{a}^{m}=A_{\mathfrak{a}, m, \kappa} \Theta_{\mathfrak{a}, \kappa}^{m}$ for some $A_{\mathfrak{a}, m, \kappa} \in \iota^{-1}\left(\overline{\mathbf{Z}}_{p}\right)$ by Theorem 7.2 It is clear that

$$
\left(\mathcal{P}_{\mathfrak{a}, \kappa}^{*} \widetilde{F}_{a}^{m}, \Theta_{\mathfrak{a}, \kappa}^{m}\right)=B^{*}(\kappa) B_{2}(\kappa) \mathbf{e}_{m}\left(-i_{\Sigma}\right)\left(F_{a}^{m}, \Theta_{\mathfrak{a}, \kappa}^{m}\right)=A_{\mathfrak{a}, m, \kappa}\left(\Theta_{\mathfrak{a}, \kappa}^{m}, \Theta_{\mathfrak{a}, \kappa}^{m}\right) .
$$

In the first equality, we used the self-adjointness of $\mathcal{P}_{\mathfrak{a}, \kappa}$. On the other hand, it is well known that $h_{\mathcal{K}}^{-}=Q \cdot \sharp\left(C_{\mathcal{K}}^{-}\left(\mathcal{O}_{\mathcal{K}}\right)\right)=Q \cdot \operatorname{vol}\left(\widehat{\mathcal{O}}_{\mathcal{K}}^{1}, d^{\times} t\right)^{-1}, Q=1$ or 2 , and hence the period integral

$$
I\left(\Theta_{\mathfrak{a}, \kappa}^{m}\right) \cdot h_{\mathcal{K}}^{-}=Q \cdot \sum_{\mathbf{c} \in I_{\mathcal{K}}^{1}} \boldsymbol{\theta}_{\mathfrak{a}, \kappa, \mathbf{c}}^{m}(0)
$$

is a sum of the evaluation of $p$-integral theta functions at the zero section. Therefore, we have the following properties for $l_{\mathfrak{a}, m, \kappa}$ :

$$
\begin{aligned}
& l_{\mathfrak{a}, m, \kappa}(F)=\iota\left(A_{\mathfrak{a}, m, \kappa} \cdot I\left(\Theta_{\mathfrak{a}, \kappa}^{m}\right) \cdot h_{\mathcal{K}}^{-}\right) \in \overline{\mathbf{Z}}_{p}, \\
& l_{\mathfrak{a}, m, \kappa}(F) \equiv l_{\mathfrak{a}, m, \kappa}(G)\left(\bmod \mathfrak{m}_{p}\right) \text { if } F \equiv G\left(\bmod \mathfrak{m}_{p}\right) .
\end{aligned}
$$

7.4. Non-vanishing of Eisenstein series modulo $\boldsymbol{p}$ (I). Let $\chi$ be a Hecke character of infinity type $k \Sigma, k>4$. Suppose that $\mathfrak{c}$ is the conductor $\mathfrak{c}(\chi)$ of $\chi$. In particular, $\chi$ is unramified at every place above $p$. We will show the non-vanishing modulo $p$ of the ordinary Eisenstein series $E^{\text {ord }}(\chi \mid \mathbf{1}, \mathfrak{n})$ defined in (5.23) under suitable hypotheses. We first introduce an auxiliary Eisenstein series $E^{\circ}(\chi \mid \mathbf{1}, \mathfrak{n})$.

Consider the following sections in $I(\chi, s)$ :

$$
\begin{aligned}
\theta_{\chi, s} & :=\bigotimes_{v \notin S_{p}} \phi_{\chi, s, v} \bigotimes_{v \in S_{p}} f_{\chi, s, v}^{0}, \\
\theta_{\chi, s}^{*} & :=\frac{(2 \pi i)^{k d}}{\Omega_{\infty}^{k \Sigma}} \cdot \frac{\Lambda_{2}^{S}(s, \chi)}{\operatorname{vol}\left(K_{P}(\mathfrak{n})\right)} \cdot \theta_{\chi, s},
\end{aligned}
$$

where $\Lambda_{2}^{S}(s, \chi)=\prod_{v \notin S} \Lambda_{2, v}(s, \chi)$ (Prop. [5.4). We note that $\theta_{\chi, s}$ differs from $\phi_{\chi, s}$ defined in Def. 5.6 only by the choice of the local section at $p$. Let $E^{\circ}(\chi, \mathfrak{n})$ be the holomorphic Siegel-Eisenstein series on $\mathbf{G}$ associated to $\left.\theta_{\chi, s}^{*}\right|_{s=0}$ defined as in (5.10).

Proposition 7.4. The Eisenstein series $E^{\circ}(\chi, \mathfrak{n})$ is defined over $\overline{\mathbf{Z}}_{(p)}$. 
Proof. It suffices to show $E^{\circ}(\chi, \mathfrak{n})$ has Fourier expansions with coefficients in $\overline{\mathbf{Z}}_{(p)}$ by $q$-expansion principle. With the notation of $\$ 5.4$ for each $\beta \in \mathcal{H}_{2}\left(\mathbf{A}_{f}\right)$ with $\operatorname{det} \beta \neq 0$, define $\mathbf{a}_{\beta}(\chi, \mathfrak{n})^{(p)}$ (the prime-to- $p$ part of $\left.\mathbf{a}_{\beta}(\chi, \mathfrak{n})\right)$ by

$$
\mathbf{a}_{\beta}^{\circ}(\chi, \mathfrak{n})^{(p)}:=\prod_{v \in S} \chi_{v}\left(\operatorname{det} \mathbf{u}^{-1}\right) \cdot \prod_{\substack{v \notin S \cup S_{p}, v(\operatorname{det} \beta)>0}} R_{\beta, v}\left(\chi_{+, v}\left(\varpi_{v}\right)\right) \cdot c_{S}(\mathcal{K}, \mathcal{L}) \cdot \mathbb{I}_{\Xi^{(p)}}(\beta),
$$

where $\Xi^{(p)}$ is the compact subset in $\mathcal{H}_{2}\left(\mathbf{A}_{\mathcal{K}, f}^{(p)}\right)$ given by

$$
\Xi^{(p)}=\prod_{v \in S} \mathcal{L}_{v}^{\vee} \times \prod_{v \notin S \cup S_{p}} \mathcal{H}_{2}\left(\mathfrak{b}_{\vartheta, v}\right)^{\vee} .
$$

By Prop. 5.4 and Prop. 5.7, $E^{\circ}(\chi, \mathfrak{n})\left(Z, g_{A}\right)$ (in the notation of loc. cit.) has the Fourier expansion

$$
\sum_{\substack{\beta \in \mathcal{H}_{2}\left(\mathcal{O}_{\mathcal{F},(p)}\right), \sigma(\beta)>0 \text { for all } \sigma \in \Sigma}} \mathbf{a}_{\beta}\left(g_{A}, E^{\circ}(\chi, \mathfrak{n})\right) q^{\beta},
$$

where $A \in \mathrm{GL}_{2}\left(\mathbf{A}_{\mathcal{K}}^{S \cup S_{p}}\right) \times \mathrm{GL}_{2}\left(\mathcal{O}_{\mathcal{K}_{p}}\right)$ and

$$
\begin{aligned}
\mathbf{a}_{\beta}\left(g_{A}, E(\chi, \mathfrak{n})\right)= & \chi^{-1}(\operatorname{det} A)|\operatorname{det} A|_{\mathbf{A}_{\mathcal{K}}}^{2} \cdot \mathbf{a}_{A^{*} \beta A}(\chi, \mathfrak{n})^{(p)} \cdot \mathbf{a}_{\beta}^{\circ}(\chi, \mathfrak{n})_{p}, \\
& \times \mathbf{a}_{\beta}^{\circ}(\chi, \mathfrak{n})_{p}:=(\operatorname{det} \beta)^{(k-2) \Sigma} \prod_{v \in S_{p}} R_{\beta, v}^{*}\left(\chi_{+, v}|\cdot|^{-2}\left(\varpi_{v}\right)\right) .
\end{aligned}
$$

It is easy to see that $\mathbf{a}_{\beta}(\chi, \mathfrak{n})^{(p)}$ belongs to $\overline{\mathbf{Z}}_{(p)}$ since $\chi\left(\mathbf{A}_{\mathcal{K}}^{(p) \times} \mathcal{O}_{\mathcal{K}_{p}}^{\times}\right) \subset \overline{\mathbf{Z}}_{(p)}^{\times}$. To see the $p$-integrality of $\mathbf{a}_{\beta}^{\circ}(\chi, \mathfrak{n})_{p}$, we note that $v_{p}\left(\chi_{+, v}|\cdot|^{-2}\left(\varpi_{v}\right)\right)=v_{p}\left(\left|\varpi_{v}\right|^{k-2}\right)$. On the other hand, by Prop. 5.4 $R_{\beta, v}^{*}(T) \in \mathbf{Z}[T]$ is a polynomial with degree less than or equal to $v(\operatorname{det} \beta)$; it follows that

$$
\mathbf{a}_{\beta}^{\circ}(\chi, \mathfrak{n})_{p}=(\operatorname{det} \beta)^{(k-2) \Sigma}|\operatorname{det} \beta|_{\mathcal{F}_{p}}^{k-2} \prod_{v \in S_{p}} R_{\beta, v}^{*}\left(\chi_{+, v}|\cdot|^{-2}\left(\varpi_{v}\right)\right) \cdot|\operatorname{det} \beta|_{\mathcal{F}_{v}}^{2-k}
$$

belongs to $\overline{\mathbf{Z}}_{(p)}$.

Let $E^{\circ}(\chi \mid \mathbf{1}, \mathfrak{n})$ be the classical Eisenstein series on $G$ associated to the pull-back section $\left.\left(\theta_{\chi, s}^{*}\right)^{p b}\right|_{s=0}$. By definition, we have

$$
\begin{aligned}
E^{\circ}(\chi \mid \mathbf{1}, \mathfrak{n})(\tau, g):= & \frac{(2 \pi i)^{k d}}{\Omega_{\infty}^{k \Sigma}} \cdot \sum_{h \in X_{W}(\mathfrak{n})} E^{\circ}(\chi, \mathfrak{n})\left(Z_{\tau}, i(g, h)\right) \\
& \cdot \chi(h),(\tau, g) \in X_{2,1} \times G\left(\mathbf{A}_{f}\right) .
\end{aligned}
$$

Then $E^{\circ}(\chi \mid \mathbf{1}, \mathfrak{n})$ is a $p$-integral modular form of weight $k I^{-}$in light of Prop. 7.4. Next we show that the ordinary projections $e . E^{\circ}(\chi \mid \mathbf{1}, \mathfrak{n})$ and $E^{\text {ord }}(\chi \mid \mathbf{1}, \mathfrak{n})$ are the same up to a $p$-adic unit.

Lemma 7.5. We have

$$
E^{\text {ord }}(\chi \mid \mathbf{1}, \mathfrak{n})=u_{1} \cdot e \cdot E^{\circ}(\chi \mid \mathbf{1}, \mathfrak{n}),
$$

where $\iota_{p}\left(u_{1}\right)$ is a p-adic unit. 
Proof. We first claim that for every $w \in \Sigma_{p}$ and $v$ the place of $\mathcal{F}$ lying below $w$,

$$
e_{v} \cdot f_{\chi, \mathbf{1}, 0, v}^{0}=\frac{L\left(-1, \chi_{\bar{w}}\right)}{L\left(0, \chi_{\bar{w}}\right)} f_{v}^{\text {ord }} \text {. }
$$

Indeed, by Lemma 6.4

$$
e_{v} \cdot f_{\chi, \mathbf{1}, 0, v}^{0}=l_{w_{2}}\left(f_{\chi, \mathbf{1}, 0, v}^{0}\right) f_{v}^{\text {ord }} .
$$

Since $f_{\chi, \mathbf{1}, s, v}^{0}$ is the standard spherical section, by the Gindikin-Karpelevič formula ([Cas80, Thm. 3.1]) we have

$$
l_{w_{2}}\left(f_{\chi, \mathbf{1}, 0, v}^{0}\right)=M_{w_{2}}\left(f_{\chi, \mathbf{1}, 0, v}^{0}\right)(1)=\frac{L\left(-1, \chi_{\bar{w}}\right)}{L\left(0, \chi_{\bar{w}}\right)} .
$$

This verifies (7.4). Using the calculations for the local pull-back sections in Prop.5.11 and Prop.6.3, we find that the ordinary Eisenstein series $e . E^{\circ}(\chi \mid \mathbf{1}, \mathfrak{n})$ is associated to the section

$$
\begin{aligned}
e .\left.\left(\theta_{\chi, s}^{*}\right)^{p b}\right|_{s=0}= & \frac{\Gamma(k)^{d} \Gamma(k-1)^{d}}{\Omega_{\infty}^{k \Sigma}(2 \pi i)^{(2 k-1) d}} \cdot L^{S \cup S_{p}}\left(-1, \chi_{+} \tau_{\mathcal{K} / \mathcal{F}}\right) L^{S \cup S_{p}}(0, \chi) \cdot f_{\chi, \mathbf{1}}^{\text {ord }} \\
& \times \prod_{w \in \Sigma_{p}, w \mid v} L\left(-1, \chi_{+, v} \tau_{\mathcal{K}_{v} / \mathcal{F}_{v}}\right) L\left(0, \chi_{w}\right) L\left(-1, \chi_{\bar{w}}\right) .
\end{aligned}
$$

Comparing e. $\left.\left(\theta_{\chi, s}^{*}\right)^{p b}\right|_{s=0}$ with the section $\left(f_{\chi, 1}^{\text {ord }}\right)^{*}$ in (6.13), we find that

$$
E^{\text {ord }}(\chi \mid \mathbf{1}, \mathfrak{n})=u_{1} \cdot e \cdot E^{\circ}(\chi \mid \mathbf{1}, \mathfrak{n}),
$$

where $u_{1}=\prod_{v \in \Sigma_{p}}\left(1-p^{d_{v}}\right) \cdot L^{\prime}\left(d_{v}=\left[\mathcal{F}_{v}: \mathbf{Q}_{p}\right]\right)$ and

$$
\begin{aligned}
L^{\prime} & =\chi_{\Sigma_{p}^{c}}(p) \cdot \prod_{w \in \Sigma_{p}, w \mid v} L\left(2, \chi_{+, v}^{-1} \tau_{\mathcal{K}_{v} / \mathcal{F}_{v}}\right)^{-1} L\left(1, \chi_{w}^{-1}\right)^{-1} L\left(-1, \chi_{\bar{w}}\right)^{-1} \\
& =\prod_{w \in \Sigma_{p}, w \mid v}\left(1-\chi_{+, v}^{-1}\left(\varpi_{v}\right)\left|\varpi_{v}\right|^{2}\right)\left(1-\chi_{w}^{-1}\left(\varpi_{v}\right)\left|\varpi_{v}\right|\right)\left(1-\chi_{\bar{w}}\left(\varpi_{v}\right)\left|\varpi_{v}\right|^{-1}\right) \cdot \chi_{\bar{w}}\left(\varpi_{v}\right) .
\end{aligned}
$$

Note that $\iota_{p}\left(L^{\prime}\right)$ is indeed a $p$-adic unit if $k>2$, since $v_{p}\left(\chi_{w}\left(\varpi_{v}\right)\right)=-k d_{v}$ and $v_{p}\left(\chi \bar{w}\left(\varpi_{v}\right)\right)=0$ for every $w \in \Sigma_{p}$. It follows that $\iota_{p}\left(u_{1}\right)$ is also a $p$-adic unit.

Let $\mathbb{E}:=E^{\circ}(\chi \mid \mathbf{1}, \mathfrak{n})$. The Eisenstein series $\mathbb{E}$ is unramified at $p$ and hence is an eigenform of the normalized Hecke operators $\left\{T_{v}\left(\alpha_{i}\right)\right\}_{i=1,2,3}$ for $v \in S_{p}$ defined in Prop. 3.15.

Lemma 7.6. The eigenvalues $\left\{\lambda_{i, v}\right\}_{i=1,2,3}$ of $\left\{T_{v}\left(\alpha_{i}\right)\right\}_{i=1,2,3}$ are given by

$$
\begin{aligned}
& \lambda_{1, v}=1+\chi_{w}^{-1}\left(\varpi_{v}\right)\left|\varpi_{v}\right|+\chi_{+, v}^{-1}\left(\varpi_{v}\right)\left|\varpi_{v}\right|^{2}, \\
& \lambda_{2, v}=\chi_{w}^{-1}\left(\varpi_{v}\right)+\left|\varpi_{v}\right|^{-1}+\chi_{+, v}^{-1}\left(\varpi_{v}\right)\left|\varpi_{v}\right|, \\
& \lambda_{3, v}=\chi_{w}^{-1}\left(\varpi_{v}\right) .
\end{aligned}
$$

It is clear that $\left\{\iota_{p}\left(\lambda_{i, v}\right)\right\}_{i=1,2,3}$ are $p$-adic units.

Proof. This is a standard local computation. For the convenience of the reader, we provide the detail for the eigenvalue of $T_{v}\left(\alpha_{1}\right)$. We shall borrow the notation from $\S 6.3$ Then $F=\mathcal{F}_{v}, \varpi=\varpi_{v}$ and $\left(\chi_{1}, \chi_{2}\right)=\left(\chi_{w}, \chi_{\bar{w}}\right)$ with $w \in \Sigma_{p}, w \mid v$. Let $f^{0}$ be the spherical function in $I_{v}(\chi, \mathbf{1}, 0)=I\left(\chi_{2}|\cdot|^{-1}, \mathbf{1}, \chi_{1}^{-1}|\cdot|\right) \otimes \chi_{2}^{-1}$ with $f^{0}(1)=1$. 
Then it suffices to show $T_{v}\left(\alpha_{1}\right) \cdot f^{0}=\lambda_{1, v} \cdot f^{0} \Longleftrightarrow T_{v}\left(\alpha_{1}\right) \cdot f^{0}(1)=\lambda_{1, v}$. With the identification $G(F)=\mathrm{GL}_{4}(F) \times F^{\times}$, we have

$$
\alpha_{1}^{\vee}=\alpha_{1}^{-1} \nu\left(\alpha_{1}\right)=(\operatorname{diag}(\varpi, \varpi, 1), \varpi) .
$$

According to (3.21), we find that

$$
\begin{aligned}
f^{0} \mid T_{v}\left(\alpha_{1}\right)(g)= & \sum_{x_{1}, x_{2} \in \mathcal{O} /(\varpi)} f^{0}\left(g\left[\begin{array}{ccc}
1 & & x_{1} \\
& 1 & x_{2} \\
& & 1
\end{array}\right]\left[\begin{array}{lll}
\varpi & & \\
& \varpi & \\
& & 1
\end{array}\right]\right) \chi_{2}^{-1}(\varpi) \\
& +\sum_{x_{3} \in \mathcal{O} /(\varpi)} f^{0}\left(g\left[\begin{array}{ccc}
1 & x_{3} & \\
& 1 & \\
& & 1
\end{array}\right]\left[\begin{array}{lll}
\varpi & & \\
& 1 & \\
& & \varpi
\end{array}\right]\right) \chi_{2}^{-1}(\varpi) \\
& +f^{0}\left(g\left[\begin{array}{ccc}
1 & & \\
& \varpi & \\
& & \varpi
\end{array}\right]\right) \chi_{2}^{-1}(\varpi) .
\end{aligned}
$$

Therefore, by the normalization in Prop. 3.15 ,

$$
\lambda_{1, v}=T_{v}\left(\alpha_{1}\right) \cdot f^{0}(1)=\left.|\varpi|^{2} \cdot f^{0}\left|T_{v}\left(\alpha_{1}\right)(1)=1+\chi_{1}^{-1}(\varpi)\right| \varpi\left|+\chi_{1}^{-1} \chi_{2}^{-1}(\varpi)\right| \varpi\right|^{2} .
$$

Lemma 7.7. Let $\Theta \in \mathbf{T}_{\text {hol,prim }}^{m}(\mathfrak{a}, \kappa)$ and let $c_{a}(\mathbb{E}):=\left(\mathbb{E}_{a}^{m}, \Theta\right)$. Put $\widetilde{\kappa}:=\kappa^{-1}|\cdot|_{\mathbf{A}_{\mathcal{K}}}^{\frac{1}{2}}$. Let $v \in S_{p}$ and $w \in \Sigma_{p}$ with $w \mid v$. If $(p, \mathfrak{f}(\mathfrak{a}, m))=1$, then we have

$$
\begin{aligned}
& c_{a}\left(T_{v}\left(\alpha_{1}\right) \cdot \mathbb{E}\right)=c_{a}\left(U_{v}\left(\alpha_{1}\right) \cdot \mathbb{E}\right)+\left|\varpi_{v}\right| \chi_{w}^{-1} \widetilde{\kappa}_{w}\left(\varpi_{v}\right) \cdot c_{a}(\mathbb{E}), \\
& c_{a}\left(T_{v}\left(\alpha_{2}\right) \cdot \mathbb{E}\right)=c_{a}\left(U_{v}\left(\alpha_{2}\right) \cdot \mathbb{E}\right)+\chi_{\bar{w}}^{-1} \widetilde{\kappa}_{\bar{w}}\left(\varpi_{v}\right) \cdot c_{a}(\mathbb{E}) .
\end{aligned}
$$

Proof. Let $\Pi_{1}$ and $\Pi_{2}$ be uniformizers of $\mathcal{K}_{w}$ and $\mathcal{K}_{\bar{w}}$ respectively. Using the formulas Prop. 3.15 and (3.21) ( $c f$. Lemma 7.6), we find that

$$
\begin{aligned}
c_{a}\left(T_{v}\left(\alpha_{1}\right) \cdot \mathbb{E}\right)= & c_{a}\left(U_{v}\left(\alpha_{1}\right) \cdot \mathbb{E}\right)+\chi^{-1}\left(\Pi_{1}\right)\left|\Pi_{1}\right| c_{a \Pi_{1}^{-1} \Pi_{2}}(\mathbb{E}) \chi^{-1}\left(\Pi_{1}^{-1} \Pi_{2}\right) \\
& +\chi^{-1}\left(\Pi_{1}\right)\left|\Pi_{1}\right|^{2} c_{a \Pi_{2}^{-1}}(\mathbb{E}), \\
c_{a}\left(T_{v}\left(\alpha_{2}\right) \cdot \mathbb{E}\right)= & c_{a}\left(U_{v}\left(\alpha_{2}\right) \cdot \mathbb{E}\right)+\chi^{-1}\left(\Pi_{1}\right) c_{a \Pi_{1} \Pi_{2}^{-1}}(\mathbb{E}) \\
& +\chi^{-1}\left(\Pi_{1}\right)\left|\Pi_{1}\right| c_{a \Pi_{1}^{-1}}(\mathbb{E}) \chi^{-1}\left(\Pi_{1}^{-1} \Pi_{2}\right) .
\end{aligned}
$$

Since $\mathfrak{f}(\mathfrak{a}, m)$ is prime to $p, \mu_{v}(\mathfrak{a}, m)=0$, and hence according to the formulas of primitive Fourier-Jacobi coefficients in [MS07, Lemma 5.2 (iii), (iv)] (note that $(\Omega, \chi)$ loc. cit. is $(\chi, \kappa))$, we have $c_{a \Pi_{1}^{-1}}(\mathbb{E})=c_{a \Pi_{2}^{-1}}(\mathbb{E})=0$ and

$$
\begin{aligned}
& c_{a \Pi_{1}^{-1} \Pi_{2}}(\mathbb{E})=\left|\Pi_{2}\right|^{\frac{1}{2}} \kappa^{-1}\left(\Pi_{1}\right) \chi\left(\Pi_{1}^{-1} \Pi_{2}\right) \cdot c_{a}(\mathbb{E}), \\
& c_{a \Pi_{1} \Pi_{2}^{-1}}(\mathbb{E})=\left|\Pi_{1}\right|^{\frac{1}{2}} \kappa^{-1}\left(\Pi_{2}\right) \chi\left(\Pi_{1} \Pi_{2}^{-1}\right) \cdot c_{a}(\mathbb{E}) .
\end{aligned}
$$

The lemma follows immediately.

Suppose that $m \in \mathcal{O}_{\mathcal{F},(p)}^{\times}$. Then we have $(p, \mathfrak{f}(\mathfrak{a}, m))=1$ by our choice of $\vartheta$ and $\mathfrak{a}$. Since $v_{p}\left(\chi_{w}^{-1} \widetilde{\kappa}_{w}\left(\varpi_{v}\right)\right)=(k-1) d_{v}$ and $v_{p}\left(\chi_{\bar{w}}^{-1} \widetilde{\kappa}_{\bar{w}}\left(\varpi_{v}\right)\right)=0$, it follows from Lemma 7.7 to $\mathbb{E}:=E^{\circ}(\chi \mid \mathbf{1}, \mathfrak{n})$ that

$$
\begin{aligned}
\iota_{p}\left(\lambda_{1, v}\right) \cdot l_{\mathfrak{a}, m, \kappa}(\mathbb{E}) & \equiv l_{\mathfrak{a}, m, \kappa}\left(U_{v}\left(\alpha_{1}\right) . \mathbb{E}\right)\left(\bmod \mathfrak{m}_{p}\right), \\
\iota_{p}\left(\lambda_{2, v}-\chi_{\bar{w}}^{-1} \widetilde{\kappa}_{\bar{w}}\left(\varpi_{v}\right)\right) \cdot l_{\mathfrak{a}, m, \kappa}(\mathbb{E}) & \equiv l_{\mathfrak{a}, m, \kappa}\left(U_{v}\left(\alpha_{2}\right) \cdot \mathbb{E}\right)\left(\bmod \mathfrak{m}_{p}\right) .
\end{aligned}
$$


Let $\lambda_{i}:=\prod_{v \in S_{p}} \lambda_{i, v}$ for $i=1,3$ and let

$$
\lambda_{2}(\kappa):=\prod_{v \in S_{p}}\left(\lambda_{2, v}-\chi_{\bar{w}}^{-1} \widetilde{\kappa}_{\bar{w}}\left(\varpi_{v}\right)\right) .
$$

Note that $U_{v}\left(\alpha_{3}\right)=T_{v}\left(\alpha_{3}\right)$. It follows from (17.5) that

$$
\iota_{p}\left(\lambda_{1} \lambda_{2}(\kappa) \lambda_{3}\right) \cdot l_{\mathfrak{a}, m, \kappa}(\mathbb{E}) \equiv l_{\mathfrak{a}, m, \kappa}\left(U_{p} \cdot \mathbb{E}\right)\left(\bmod \mathfrak{m}_{p}\right) \quad\left(U_{p}=\prod_{i=1,2,3, v \in S_{p}} U_{v}\left(\alpha_{i}\right)\right) .
$$

Therefore, by Lemma 7.5 and the definition $e=\lim _{n \rightarrow \infty} U_{p}^{n !}$ we have proved the following key proposition.

Proposition 7.8. If $m \in \mathcal{O}_{\mathcal{F},(p)}^{\times}$and $\iota_{p}\left(\lambda_{2}(\kappa)\right)$ is a p-adic unit, then

$$
l_{\mathfrak{a}, m, \kappa}\left(E^{o r d}(\chi \mid \mathbf{1}, \mathfrak{n})\right) \not \equiv 0\left(\bmod \mathfrak{m}_{p}\right) \Longleftrightarrow l_{\mathfrak{a}, m, \kappa}\left(E^{\circ}(\chi \mid \mathbf{1}, \mathfrak{n})\right) \not \equiv 0\left(\bmod \mathfrak{m}_{p}\right) .
$$

7.5. Results of Murase and Sugano. Let $w_{\mathcal{K}}$ be the cardinality of the torsion subgroup of $\mathcal{O}_{\mathcal{K}}^{\times}$. Define the subset $T$ of prime ideals of $\mathcal{O}_{\mathcal{F}}$ by

$$
T=\left\{\mathfrak{q} \mid \mathrm{N}_{\mathcal{F} / \mathbf{Q}}(\mathfrak{q}) \not \equiv \pm 1(\bmod p),\left(\mathfrak{q}, 2 p D_{\mathcal{K}} \vartheta \mathfrak{c}\right)=1, \mathrm{~N}_{\mathcal{F} / \mathbf{Q}}(\mathfrak{q})-1>w_{\mathcal{K}}\right\} .
$$

Let $\mathcal{E}$ denote the set consisting of triples $(\mathfrak{a}, m, \kappa), m \in \mathcal{O}_{\mathcal{F},(p),+}^{\times}$, a prime-to- $p$ $\mathcal{O}_{\mathcal{F}}$-fractional ideal $\mathfrak{a}$ and $\kappa \in \mathcal{X}_{0}(\mathfrak{a}, m)$ such that $\mathfrak{f}(\mathfrak{a}, m)=\mathfrak{a}_{\mathcal{D}} \prod_{i} \mathfrak{q}_{i}$, where $\mathfrak{q}_{i}$ are

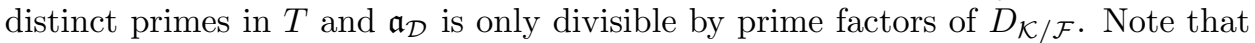
if $(\mathfrak{a}, m, \kappa) \in \mathcal{E}$, then $p \nmid \mathfrak{a} \overline{\mathfrak{a}} B_{1}(\kappa) B_{2}(\kappa)$. Suppose that we are given a specific triple $(\mathfrak{a}, m, \kappa) \in \mathcal{E}$, which will be determined in Prop. 7.13. In addition to (a1) and (a2), we assume the auxiliary ideal $\mathfrak{n}$ is chosen to be sufficiently small such that for every $v \in S$

$$
\Theta_{\mathfrak{a}, \kappa}^{m}(r n(b, x))=\Theta_{\mathfrak{a}, \kappa}^{m}(r) \text { for every } x, b \in \mathfrak{a}_{v} \mathfrak{n}_{v} \text { and } r \in R(\mathbf{A}) .
$$

It is obvious that $\mathfrak{n}$ only depends on $m$ and $\left(\mathfrak{a}_{v}, \kappa_{v}\right)_{v \in S}$.

Let $\mathfrak{l}=\mathfrak{L} \overline{\mathfrak{L}}$ be a prime in Remark 7.1 and $\varpi_{\mathfrak{L}}$ be a uniformizer of $\mathcal{K}_{\mathfrak{L}}$. Let $\nu \in \mathfrak{X}_{\mathfrak{l}}^{-}$be an anticyclotomic character of conductor $\mathfrak{l}^{n}$. Let $b_{\vartheta}=\left(b_{\vartheta, v}\right) \in \mathbf{A}_{f}^{\times}$such that $\left(\vartheta^{-1} \mathcal{D}_{\mathcal{K} / \mathcal{F}}^{-1}\right) \cap \mathcal{F}=b_{\vartheta} \mathcal{O}_{\mathcal{F}, f} \cap \mathcal{F}$. Put

$$
u_{\chi}^{\prime}=\left|D_{\mathcal{F}}\right|_{\mathbf{R}}^{-\frac{1}{2}} \mathrm{~N}_{\mathcal{K} / \mathbf{Q}}(m)^{k-1} \cdot \prod_{v \notin S} \chi\left(b_{\vartheta, v}\right)\left|\mathfrak{f}\left(\mathfrak{a} \mathfrak{L}^{n}, m\right) b_{\vartheta, v}^{-1}\right|_{\mathcal{F}_{v}} \cdot \prod_{v \in S}\left|\mathfrak{n}^{3} \vartheta^{2}\right|_{\mathcal{F}_{v}} .
$$

The following result is essentially due to Murase and Sugano.

Theorem 7.9. Let $\mathbb{E}:=E^{\circ}(\chi \mid \mathbf{1}, \mathfrak{n})$. If $\Theta \in \mathbf{T}_{\text {hol,prim }}^{m}\left(\mathfrak{a} \mathfrak{L}^{n}, \kappa \nu\right)$, then we have

$$
\mathbf{e}_{m}\left(-i_{\Sigma}\right) \cdot\left(\mathbb{E}_{\alpha}^{m}, \Theta\right)=u_{2} \cdot \frac{(4 \pi)^{d} \cdot \Gamma(k-1)^{d}}{\Omega_{\infty}^{k \Sigma}} \cdot L^{S}\left(-\frac{1}{2}, \chi \kappa \nu\right) \cdot \overline{I(\Theta)},
$$

where $\alpha=a \varpi_{\mathfrak{L}}^{n}$ and $u_{2}:=u_{\chi}^{\prime} \chi_{f}^{-c}(\alpha)|\alpha|_{\mathbf{A}_{\mathcal{K}, f}}$ is a p-adic unit.

Proof. This theorem was proved in [MS02, Thm. 4.4] for the special case where $\chi$ is unramified everywhere and $\mathfrak{n}=\mathcal{O}_{\mathcal{F}}$. Following the computations of Murase and Sugano, we sketch the proof of the general case. The starting point is the following integral expression of Fourier-Jacobi coefficients of Eisenstein series. 
Lemma 7.10 (Lemma 6.6 MS02]). Let $E=E_{\mathbf{A}}(-, f)$ be the adelic Eisenstein series associated to a decomposable section $f=\otimes_{v} f_{v} \in I(\chi, \mathbf{1}, 0)$ and $\Theta \in \mathbf{T}_{\text {hol,prim }}^{m}$ $\left(\mathfrak{a}^{n}, \kappa \nu\right)$. Then we have

$$
\left(E_{\alpha}^{m}, \Theta\right)=\chi_{f}^{-c}(\alpha)|\alpha|_{\mathbf{A}_{\mathcal{K}}} \cdot \int_{\mathcal{K}^{1} \backslash \mathcal{K}_{\mathbf{A}}^{1}} \mathcal{T}_{\kappa \nu}^{m}\left(W_{\chi, \Phi, f}\right)(t) d^{\times} t,
$$

where $\Phi=\otimes \Phi_{v} \in V_{h o l}^{m}$ such that $\mathcal{T}_{\kappa \nu}^{m}(\Phi)=\Theta$ and $W_{\chi, \Phi, f}=\otimes_{v} W_{\chi_{v}, \Phi_{v}, f_{v}}$ is a decomposable function on $\mathbf{A}_{\mathcal{K}}$. For each place $v$ of $\mathcal{F}$, we have

$$
W_{\chi_{v}, \Phi_{v}, f_{v}}\left(z_{v}\right)=\int_{\mathcal{K}_{v}} J_{\chi_{v}, f_{v}}^{\alpha_{v}}\left(b_{v}\right) \overline{\rho\left(n\left(b_{v}, 0\right)\right) \Phi_{v}\left(z_{v}\right)} d b_{v}
$$

and

$$
\begin{aligned}
J_{\chi_{v}, f_{v}}^{\alpha_{v}}\left(b_{v}\right) & =\int_{\mathcal{F}_{v}} f_{v}\left(\Upsilon_{0} n\left(\alpha_{v}^{-1} b_{v}, x_{v}\right)\right) \psi_{m}\left(-\mathrm{N}_{\mathcal{K} / \mathcal{F}}\left(\alpha_{v}\right) x_{v}\right) d x_{v}, \Upsilon_{0} \\
& =\left[\begin{array}{rrr} 
& 1 & \\
-1 & &
\end{array}\right]=-w_{l} .
\end{aligned}
$$

Define the section $f:=f_{\infty, k} \otimes_{v \in \mathbf{h}, v \notin S} f_{\chi, 0, v}^{0} \otimes_{v \in S} f_{\chi, \mathbf{1}, 0, v}^{\mathfrak{n}}$. If $v$ is archimedean, then by [MS02, Lemma 6.8] we have

$$
W_{\chi_{v}, \Phi_{v}, f_{v}}=\frac{(2 \pi i)^{k d}}{\Gamma(k)^{d}} \cdot\left|D_{\mathcal{F}}\right|_{\mathbf{R}}^{-\frac{1}{2}} \mathrm{~N}_{\mathcal{K} / \mathbf{Q}}(m)^{k-1} \mathbf{e}_{m}\left(i_{\Sigma}\right) \cdot \bar{\Phi}_{v} .
$$

For each finite $v \notin S, f_{v}$ is the spherical section and $v$ is unramified in $\mathcal{K}$. By [MS02, Prop. 10.2], we have

$$
W_{\chi_{v}, \Phi_{v}, f_{v}}=\chi\left(b_{\vartheta, v}\right)\left|b_{\vartheta, v}\right|^{-1} \cdot \frac{L^{S}\left(-\frac{1}{2}, \chi_{v} \kappa_{v} \nu_{v}\right) L\left(-1, \chi_{+, v} \tau_{\mathcal{K}_{v} / \mathcal{F}_{v}}\right)}{L\left(0, \chi_{v}\right)} \cdot \Phi_{v} .
$$

Suppose that $v \in S$. Note that

$$
\Upsilon_{0} n\left(\alpha_{v}^{-1} b_{v}, x_{v}\right) \in P(F) w_{l} D_{V}\left(\mathfrak{n}_{v}\right) \Longleftrightarrow \alpha_{v}^{-1} b_{v} \in \mathfrak{n}_{v} \vartheta, x_{v} \in \mathfrak{n}_{v} .
$$

As the prime $\ell$ is prime to each place in $S$, by the multiplicity one for (local) primitive theta functions and (a3), we have $J_{\chi_{v}, f_{v}}^{\alpha_{v}}=\mathbb{I}_{\mathfrak{a}_{v} \mathfrak{n}_{v} \vartheta}$ and $\rho\left(n\left(b_{v}, 0\right)\right) \Phi_{v}=\Phi_{v}$ for $b_{v} \in \mathfrak{a}_{v} \mathfrak{n}_{v}$. Hence,

$$
W_{\chi_{v}, \Phi_{v}, f_{v}}=\left|\mathfrak{n}^{3} \vartheta^{2}\right| \cdot \bar{\Phi}_{v} .
$$

Combining these formulas, we find that

$$
\mathcal{T}_{\kappa \nu}^{m}\left(W_{\chi, \Phi, f}\right)=u_{\chi}^{\prime} \cdot \mathbf{e}_{m}\left(i_{\Sigma}\right) \cdot \frac{(2 \pi i)^{k d}}{\Gamma(k)^{d}} \cdot \frac{L^{S}\left(-\frac{1}{2}, \chi \kappa \nu\right)}{L^{S}\left(-1, \chi_{+} \tau_{\mathcal{K} / \mathcal{F}}\right) L^{S}(0, \chi)} \cdot \bar{\Theta} .
$$

Note that the Eisenstein series $\mathbb{E}$ is associated to the section $\left.\left(\theta_{\chi, s}^{*}\right)^{p b}\right|_{s=0}$ and that

$$
\left.\left(\theta_{\chi, s}^{*}\right)^{p b}\right|_{s=0}=\frac{(2 \pi i)^{k d}}{\Omega_{\infty}^{k \Sigma}} \cdot \Lambda_{2, \infty}(0, \chi) \cdot L^{S}\left(-1, \tau_{\mathcal{K} / \mathcal{F}} \chi_{+}\right) L^{S}(0, \chi) \cdot f .
$$

Therefore, the theorem follows from (7.9) and Lemma 7.10

We recall the following result due to Tonghai Yang [Yan97] (cf. [MS02, Thm. 4.5]). 
Theorem 7.11. If $\Theta \in \mathbf{T}_{\text {hol,prim }}^{m}\left(\mathfrak{a l}^{n}, \kappa \nu\right)$, then

$$
|I(\Theta)|^{2}=\frac{1}{2 L\left(1, \tau_{\mathcal{K} / \mathcal{F}}\right)} \cdot L\left(\frac{1}{2}, \kappa \nu\right)(\Theta, \Theta) \cdot B_{1}(\kappa \nu)^{-1} B_{2}(\kappa \nu)^{-1} .
$$

By Theorem 7.9. Theorem 7.11 and the well-known analytic class number formula

we find that

$$
2 L\left(1, \tau_{\mathcal{K} / \mathcal{F}}\right)=(2 \pi)^{d}\left|D_{\mathcal{F}}\right|_{\mathbf{R}}^{\frac{1}{2}}\left|D_{\mathcal{K}}\right|_{\mathbf{R}}^{-\frac{1}{2}}\left[\mathcal{O}_{\mathcal{K}}^{\times}: \mathcal{O}_{\mathcal{F}}^{\times}\right]^{-1} h_{\mathcal{K}}^{-},
$$

$$
\begin{aligned}
l_{\mathfrak{a} l^{n}, m, \kappa \nu}\left(E^{\circ}(\chi \mid \mathbf{1}, \mathfrak{n})\right)= & \iota_{p}\left(\frac{\Gamma(k-1)^{d} L^{S}\left(0, \chi \widetilde{\kappa}^{-1} \nu\right)}{\Omega_{\infty}^{(k-1) \Sigma}} \cdot \frac{\Gamma(1)^{d} L\left(0, \widetilde{\kappa} \nu^{-1}\right)}{\Omega_{\infty}^{1 \cdot \Sigma}}\right) \\
& \cdot \iota_{p}\left(B_{2}(\kappa \nu) h_{\mathcal{K}}^{\overline{\mathcal{C}}}\right) \cdot u_{3} .
\end{aligned}
$$

for some $p$-adic unit $u_{3}$. Here $\widetilde{\kappa}=\kappa^{-1}|\cdot|_{\mathbf{A}_{\mathcal{K}}}^{\frac{1}{2}}$, and we have used the identity $L\left(\frac{1}{2}, \kappa \nu\right)=L\left(\frac{1}{2}, \kappa^{-c} \nu^{-c}\right)=L\left(\frac{1}{2}, \kappa^{-1} \nu^{-1}\right)$.

7.6. Non-vanishing of Eisenstein series modulo $\boldsymbol{p}$ (II). To study the nonvanishing of $E^{\text {ord }}(\chi \mid \mathbf{1}, \mathfrak{n})$ modulo $p$, we make the following hypothesis for $\chi$ :

Hypothesis 7.12. There exist $(\mathfrak{a}, m, \kappa) \in \mathcal{E}$ and $\mathfrak{l} \in T$ such that

- $\mathfrak{l}$ has degree one over $\mathbf{Q}$, splits in $\mathcal{K}$ and $\mathfrak{l} \nmid \mathfrak{c}(\kappa)$.

- The local $L$-factor $L\left(0, \chi_{v} \widetilde{\kappa}_{v}^{-1}\right)^{-1} \not \equiv 0(\bmod \mathfrak{m})$ for every $v \mid D_{\mathcal{K} / \mathcal{F}}$.

- There are infinitely many anticyclotomic finite order characters $\nu \in \mathfrak{X}_{\mathfrak{l}}^{-}$ such that

$$
\frac{\Gamma(k-1)^{d} L\left(0, \chi \widetilde{\kappa}^{-1} \nu\right)}{\Omega_{\infty}^{(k-1) \Sigma}} \cdot \frac{\Gamma(1)^{d} L\left(0, \widetilde{\kappa} \nu^{-1}\right)}{\Omega_{\infty}^{1 \cdot \Sigma}} \not \equiv 0(\bmod \mathfrak{m}) .
$$

Proposition 7.13. Suppose further that $p \nmid h_{\mathcal{K}}^{-}$and $\chi$ satisfies Hypothesis 7.12 , Then there exist $(\mathfrak{a}, m, \kappa) \in \mathcal{E}$ and an auxiliary ideal $\mathfrak{n}$ satisfying (a1)-(a3) such that

$$
l_{\mathfrak{a}, m, \kappa}\left(E^{o r d}(\chi \mid \mathbf{1}, \mathfrak{n})\right) \not \equiv 0\left(\bmod \mathfrak{m}_{p}\right) .
$$

Proof. Let $(\mathfrak{a}, m, \kappa)$ and $\mathfrak{l}$ be as in Hypothesis 7.12. Write $\mathfrak{l}=\mathfrak{L} \overline{\mathfrak{L}}$ in $\mathcal{K}$. Choose an integral ideal $\mathfrak{n}$ of $\mathcal{O}_{\mathcal{F}}$ such that $\mathfrak{n}$ satisfies (a1), (a2) and (a3) according to this triple $(\mathfrak{a}, m, \kappa) \in \mathcal{E}$. We claim that there exists some $\nu \in \mathfrak{X}_{\mathfrak{l}}^{-}$of conductor $\mathfrak{l}^{n}$ such that

(i) $\iota_{p}\left(\lambda_{2}(\kappa \nu)\right) \not \equiv 0\left(\bmod \mathfrak{m}_{p}\right)$, and

(ii) $A_{\mathfrak{a} \mathfrak{L}^{n}, m, \kappa \nu}=l_{\mathfrak{a} \mathfrak{L}^{n}, m, \kappa \nu}\left(E^{\circ}(\chi \mid \mathbf{1}, \mathfrak{n})\right) \not \equiv 0\left(\bmod \mathfrak{m}_{p}\right)$.

Then it follows from Prop. 7.8 that $\left(\mathfrak{a} \mathfrak{L}^{n}, m, \kappa \nu\right) \in \mathcal{E}$ and $\mathfrak{n}$ enjoy the desired properties in the proposition.

Now we prove the claim. Note that $p \nmid B_{1}(\kappa \nu)$ for all $\nu \in \mathfrak{X}_{\mathfrak{\digamma}}^{-}$since $(\mathfrak{a}, m, \kappa) \in \mathcal{E}$ and $\mathfrak{l} \in T$. By the formula (7.10) and Hypothesis 7.12, there are infinitely many $\nu \in \mathfrak{X}_{\mathfrak{l}}^{-}$such that

$$
L_{S}\left(0, \chi \widetilde{\kappa}^{-1} \nu\right) \cdot A_{\mathfrak{a} \mathfrak{L}^{n}, m, \kappa \nu} \not \equiv 0\left(\bmod \mathfrak{m}_{p}\right) \quad\left(L_{S}\left(0, \chi \widetilde{\kappa}^{-1}\right)=\prod_{v \mid \mathfrak{n}} L\left(0, \chi_{v} \widetilde{\kappa}_{v}^{-1}\right)\right) .
$$

Since $\mathfrak{c}=\mathfrak{c}(\chi)$ is prime to $\mathfrak{c}(\kappa)$ and $\mathfrak{n}$ satisfies $($ a1 $), L_{S}\left(0, \chi \widetilde{\kappa}^{-1} \nu\right)$ is a product of local Euler factors at ramified or split primes. Since $L\left(0, \chi_{v} \widetilde{\kappa}_{v}^{-1} \nu_{v}\right)^{-1}=1-\chi \widetilde{\kappa}^{-1} \nu\left(\delta_{v}\right)=$ 
$1-\chi \widetilde{\kappa}^{-1}\left(\delta_{v}\right) \not \equiv 0(\bmod \mathfrak{m})$ for $v \mid D_{\mathcal{K} / \mathcal{F}}$ by the choice of $\kappa$, and Frobenius elements at split primes in $S \cup S_{p}$ generate a non-trivial subgroup in $\operatorname{Gal}\left(\mathcal{K}_{\infty, 1}^{-} / \mathcal{K}\right) \stackrel{\sim}{\rightarrow} \mathbf{Z}_{\ell}$, there are only finitely many $\nu$ such that $L_{S}\left(0, \chi \widetilde{\kappa}^{-1} \nu\right)^{-1} \equiv 0(\bmod \mathfrak{m})$ and $\lambda_{2}(\kappa \nu)=$ $\prod_{v \in S_{p}}\left(\lambda_{2, v}-\chi_{\bar{w}}^{-1} \widetilde{\kappa}_{\bar{w}} \nu_{\bar{w}}\left(\varpi_{v}\right)\right) \equiv 0(\bmod \mathfrak{m})$. Hence there exists some $\nu \in \mathfrak{X}_{\mathfrak{l}}^{-}$such that (i) and (ii) hold. This completes the proof.

After preparing some elementary lemmas on the construction of triples $(\mathfrak{a}, m, \kappa) \in$ $\mathcal{E}$ with prescribed properties at ramified places, we verify Hypothesis 7.12 for a certain class of $\chi$ using the main result in [Hsi12] on the non-vanishing modulo $p$ of Hecke $L$-values for CM fields.

Lemma 7.14. For $\kappa \in \mathcal{X}$ and $m \in \mathcal{F}_{+}$, we have

(1) $W_{m}\left(\kappa_{v}\right)= \pm \kappa_{v}(\vartheta)$.

(2) If $v$ is split, $W_{m}\left(\kappa_{v}\right)=\kappa_{v}(\vartheta)$.

(3) If $v$ is inert, $W_{m}\left(\kappa_{v}\right)=(-1)^{\left(a\left(\kappa_{v}\right)+\mu_{v}(\mathfrak{a}, m)\right)} \kappa_{v}(\vartheta)$.

(4) If $\sigma \in \Sigma$ is archimedean, $W_{m}\left(\kappa_{\sigma}\right)=\kappa_{\sigma}(\vartheta)$.

Proof. If $v$ is a finite place, this is proved in [MS00, Prop. 3.7]. If $\sigma \in \Sigma$ is archimedean, then $W_{m}\left(\kappa_{\sigma}\right)=i=\kappa_{\sigma}(\vartheta)$ since $i \sigma(\vartheta)>0$ for $\sigma \in \Sigma$ by our choice of $\vartheta$.

Lemma 7.15. Suppose $p \nmid 6 \cdot D_{F}$. Let $\mathcal{K}_{\mathcal{D}}=\prod_{v \mid D_{\mathcal{K} / \mathcal{F}}} \mathcal{K}_{v}$ and $\mathcal{F}_{\mathcal{D}}=\prod_{v \mid D_{\mathcal{K} / \mathcal{F}}} \mathcal{F}_{v}$. For each character $\tau^{\sharp}: \mathcal{K}_{\mathcal{D}}^{\times} \rightarrow \mathbf{C}^{\times}$such that $\left.\tau^{\sharp}\right|_{\mathcal{F}_{\mathcal{D}}^{\times}}=\tau_{\mathcal{K} / \mathcal{F}}$, there exists a triple $(\mathfrak{a}, m, \kappa) \in \mathcal{E}$ such that $\left.\kappa\right|_{\mathcal{K}_{\mathcal{D}}^{\times}}=\tau^{\sharp}$.

Proof. Let $\mathfrak{a}_{\mathcal{D}}$ be the conductor of $\tau^{\sharp}$ and let $\mathfrak{a}_{\mathcal{D}}^{\prime}$ be the integral ideal of $\mathcal{O}_{\mathcal{K}}$ such that for each finite place $w$ of $\mathcal{K}$,

$$
w\left(\mathfrak{a}_{\mathcal{D}}^{\prime}\right)= \begin{cases}w\left(\mathfrak{a}_{\mathcal{D}}\right)-w\left(D_{\mathcal{K} / \mathcal{F}}\right) & \text { if } w\left(\mathfrak{a}_{D}\right) \geq w\left(D_{\mathcal{K} / \mathcal{F}}\right)>0, \\ 0 & \text { otherwise. }\end{cases}
$$

Since $p>3$ is unramified in $\mathcal{K}$, by Chebotarev density theorem there exist $m_{0} \in \mathcal{F}_{+}$ and $\mathfrak{a}_{0}$ such that $\mathfrak{f}\left(\mathfrak{a}_{0}, m_{0}\right)=\mathfrak{q}_{1} \mathfrak{q}_{2} \mathfrak{a}_{\mathcal{D}}^{\prime}$ for some $\mathfrak{q}_{1}, \mathfrak{q}_{2} \in T$ with $\mathfrak{q}_{2}$ split, and if $\mathcal{D}_{\mathcal{K} / \mathcal{F}} \neq(1)$ we can choose $\mathfrak{q}_{1}$ to be split or inert. Note that $\mathcal{X}_{0}(\mathfrak{a}, m)=$ $\mathcal{X}_{0}\left(\mathfrak{a N}_{\mathcal{K} / \mathcal{F}}(\alpha), \mathrm{N}_{\mathcal{K} / \mathcal{F}}(\alpha)^{-1} m\right)$ for $\alpha \in \mathcal{K}^{\times}$and $p$ satisfies the ordinary condition, so we can further choose $m_{0} \in \mathcal{O}_{\mathcal{F},(p),+}^{\times}$by the weak approximation theorem.

We claim that there exists $\kappa \in \mathcal{X}$ such that $\left.\kappa\right|_{\mathcal{K}_{\mathcal{D}}^{\times}}=\tau^{\sharp}$, the global root number $W(\kappa)=\prod_{v} W_{m_{0}}\left(\kappa_{v}\right)=1$ and the conductor $\mathfrak{c}(\kappa)=\mathfrak{q}_{1} \mathfrak{q}_{2} \mathfrak{a}_{\mathcal{D}}$. Suppose the claim is true. We choose $u=\left(u_{v}\right) \in \mathbf{A}$ such that $u_{v}=1$ if $v \mid \infty, u_{v} \in \mathcal{O}_{\mathcal{F}_{v}}^{\times}$if $v \in \mathbf{h}$ and $W_{m_{0}}\left(\kappa_{v}\right)=\kappa_{v}\left(u_{v} \vartheta\right)=\tau_{\mathcal{K} / \mathcal{F}}\left(u_{v}\right) \kappa_{v}(\vartheta)$ at all $v \mid D_{\mathcal{K} / \mathcal{F}}$. Thus by Lemma 7.14 (4) and $W(\kappa)=1$, we find that $\tau_{\mathcal{K} / \mathcal{F}}(u)=1$. Thus there exist $m_{1} \in \mathcal{F}_{+}$and $a_{1} \in \mathbf{A}_{\mathcal{K}}^{\times}$such that $m_{1} N_{\mathcal{K} / \mathcal{F}}\left(a_{1}\right)=u$. Since $v \mid p$ splits in $\mathcal{K}$, we may further assume $m_{1} \in \mathcal{O}_{\mathcal{F},(p),+}^{\times}$. Put $m=m_{0} m_{1}$ and $\mathfrak{a}=\mathfrak{a}_{1} \mathfrak{a}_{0}$ for $\mathfrak{a}_{1}=\mathcal{K} \cap a_{1} \mathcal{O}_{\mathcal{K}, f}$. Then $\mathfrak{f}(\mathfrak{a}, m)=\mathfrak{f}\left(\mathfrak{a}_{0}, m_{0}\right)=\mathfrak{q}_{1} \mathfrak{q}_{2} \mathfrak{a}_{\mathcal{D}}^{\prime}$. By Lemma 7.14 and the definition of $\mathfrak{a}_{\mathcal{D}}^{\prime}$, we find that $\kappa \in \mathcal{X}_{0}(\mathfrak{a}, m)$.

Now we prove the claim. Let

$$
U^{\prime}:=\mathcal{K}^{\times} \cdot \mathbf{A}^{\times} \cdot\left(\mathcal{K}_{\infty}^{\times} \times \mathcal{K}_{\mathcal{D}}^{\times} \times \prod_{v \nmid \mathfrak{q}_{1} \mathfrak{q}_{2} D_{\mathcal{K} / \mathcal{F}}} \mathcal{O}_{\mathcal{K}_{v}}^{\times} \times \prod_{i=1}^{2}\left(1+\mathfrak{q}_{i}\right)\right) .
$$


Define a character $\epsilon$ on $U^{\prime}$ by

$$
\begin{aligned}
& \text { - } \boldsymbol{\epsilon}_{\infty}(z)=\prod_{\sigma \in \Sigma} \frac{\left|z_{\sigma}\right|}{z_{\sigma}}, z=\left(z_{\sigma}\right)_{\sigma \in \Sigma} \in\left(\mathcal{K} \otimes_{\mathbf{Q}} \mathbf{R}\right)^{\times} . \\
& \text {- } \boldsymbol{\epsilon}_{v}=1 \text { for finite } v \nmid D_{\mathcal{K} / \mathcal{F}} \text {, and } \boldsymbol{\epsilon}_{\mathcal{K}_{\mathcal{D}}^{\times}}=\tau^{\sharp} . \\
& \text { - } \boldsymbol{\epsilon}_{\mathcal{K}^{\times}}=1 \text { and }\left.\boldsymbol{\epsilon}\right|_{\mathbf{A}^{\times}}=\tau_{\mathcal{K} / \mathcal{F}} \text {. }
\end{aligned}
$$

One can show that $\boldsymbol{\epsilon}$ is a well-defined character using the assumption that $\left(\mathfrak{q}_{1} \mathfrak{q}_{2}, w_{\mathcal{K}}\right)=1$. Also, from the assumption that $\mathrm{N}_{\mathcal{K} / \mathbf{Q}}\left(\mathfrak{q}_{i}\right)-1>w_{\mathcal{K}}$, it is not difficult to deduce that the image of $\mathcal{O}_{\mathcal{K}_{\mathfrak{q}_{i}}}^{\times}$is a non-trivial subgroup in $\mathbf{A}_{\mathcal{K}}^{\times} / U_{i}$, $U_{i}:=U^{\prime} \mathcal{O}_{\mathcal{K}_{\mathfrak{q}_{j}}}^{\times}$and $\{i, j\}=\{1,2\}$. Therefore, there exists a Hecke character $\kappa$ of $\mathcal{K}^{\times}$such that $\left.\kappa\right|_{U^{\prime}}=\boldsymbol{\epsilon}$ and $\mathfrak{c}(\kappa)=\mathfrak{q}_{1} \mathfrak{q}_{2} \mathfrak{a}_{\mathcal{D}}$. If $\mathcal{D}_{\mathcal{K} / \mathcal{F}}=(1)$, then $W(\kappa)=1$ in virtue of Lemma 7.14. Suppose $D_{\mathcal{K} / \mathcal{F}} \neq(1)$. Put $a_{\mathfrak{q}_{1}}=1$ if $\mathfrak{q}_{1}$ is inert and $a_{\mathfrak{q}_{1}}=0$ if $\mathfrak{q}_{1}$ is split. It follows from [Roh82, Prop. 6, Prop. 11] and Lemma 7.14 that

$$
\begin{aligned}
W(\kappa)= & \prod_{v} \frac{W_{m_{0}}\left(\kappa_{v}\right)}{W_{m_{0}}\left(\tau_{\mathcal{K}_{v} / \mathcal{F}_{v}}\right)} \\
= & \prod_{v \mid D_{\mathcal{K} / \mathcal{F}}} W\left(\tau_{v}^{\sharp}, \tau_{\mathcal{K}_{v} / \mathcal{F}_{v}}\right) \cdot \frac{\kappa_{\mathfrak{q}_{1}}(\vartheta)}{\tau_{\mathcal{K}_{\mathfrak{q}_{1}} / \mathcal{F}_{\mathfrak{q}_{1}}}\left(m_{0}\right)} \cdot \kappa_{\mathfrak{q}_{2}}(\vartheta) \\
& \times\left(W\left(\tau_{v}^{\sharp}, \tau_{\mathcal{K}_{v} / \mathcal{F}_{v}}\right):=\frac{W_{m_{0}}\left(\tau_{v}^{\sharp}\right)}{W_{m_{0}}\left(\tau_{\mathcal{K}_{v} / \mathcal{F}_{v}}\right)}\right) \\
= & (-1)^{a_{\mathfrak{q}_{1}}} A\left(\tau^{\sharp}, \vartheta\right),
\end{aligned}
$$

where

$$
A\left(\tau^{\sharp}, \vartheta\right):=\prod_{v \mid D_{\mathcal{K} / \mathcal{F}}} W\left(\tau_{v}^{\sharp}, \tau_{\mathcal{K}_{v} / \mathcal{F}_{v}}\right) \cdot \prod_{v \nmid D_{\mathcal{K} / \mathcal{F}}, v \mid \vartheta} \boldsymbol{\epsilon}_{v}\left(\vartheta^{-1}\right) \prod_{v \mid D_{\mathcal{K} / \mathcal{F}}} \tau_{v}^{\sharp}\left(\vartheta^{-1}\right) .
$$

To deduce the last equality, we have used the fact that $\vartheta \in \mathcal{O}_{\mathcal{K}_{v}}^{\times} \mathcal{F}_{v}^{\times}$for all $v$ unramified in $\mathcal{K},\left(\mathfrak{q}_{1} \mathfrak{q}_{2}, \vartheta D_{\mathcal{K}}\right)=1$ and $v_{\mathfrak{q}_{1}}\left(m_{0}\right) \equiv 1(\bmod 2)$. A key observation is that $A\left(\tau^{\sharp}, \vartheta\right)$ does not depend on the choice of $\mathfrak{q}_{i} \in T$. Since $W(\kappa)$ must be \pm 1 , the number $A\left(\tau^{\sharp}, \vartheta\right)= \pm 1$, and we can manage $\mathfrak{q}_{1}$ to be inert or split so that $W(\kappa)=1$.

We introduce an invariant $\mu_{p}(\lambda)$ for a Hecke character $\lambda$ of $\mathcal{K}^{\times}$. Write the conductor $\mathfrak{c}(\lambda)=\mathfrak{c}(\lambda)^{+} \mathfrak{c}(\lambda)^{-}$, where $\mathfrak{c}(\lambda)^{-}$(resp. $\left.\mathfrak{c}(\lambda)^{+}\right)$is a product of non-split prime factors (resp. split prime factors) over $\mathcal{F}$. We put

$$
\mu_{p}(\lambda):=\sum_{w \mid \mathfrak{c}(\lambda)^{-}} \inf _{x \in \mathcal{K}_{v}^{\times}} v_{p}\left(\lambda_{w}(x)-1\right)
$$

Proposition 7.16. If $\mu_{p}(\chi)=0$, then $\chi$ satisfies Hypothesis [7.12.

Proof. By Lemma 7.15, there exists $(\mathfrak{a}, m, \kappa) \in \mathcal{E}$ such that $L\left(0, \chi_{v} \widetilde{\kappa}_{v}^{-1}\right)^{-1} \not \equiv$ $0(\bmod \mathfrak{m})$ for every $v \mid D_{\mathcal{K} / \mathcal{F}}$. By the choice of $\kappa, \widetilde{\kappa}$ is self-dual with the global root number $W(\kappa)=1$. On the other hand, the $p$-adic avatar of $\chi \widetilde{\kappa}^{-1}$ is not residually self-dual since $\chi$ is unramified at $p>3$. We note that $\mu_{p}(\widetilde{\kappa})=\mu_{p}\left(\chi \widetilde{\kappa}^{-1}\right)=\mu_{p}(\chi)=$ 0 since the conductors of $\kappa$ and $\chi$ only have common divisor at ramified primes and $p>2$. Let $\mathfrak{l} \in T$ be a prime of $\mathcal{F}$ such that $\mathfrak{l} \nmid \mathfrak{c}(\kappa)$ has degree one over $\mathbf{Q}$. We apply Hsi12, Cor. 6.5] to $\left(\chi \widetilde{\kappa}^{-1}, \mathfrak{l}\right)$ and [Hsi12, Thm. 6.8] together with Hsi13, Remark $6.4]$ to $(\widetilde{\kappa}, \mathfrak{l})$ respectively, and the proposition follows. 
7.7. $\boldsymbol{\Lambda}$-adic Hecke algebra for $\boldsymbol{U}(\mathbf{2}, \mathbf{1})$. Suppose that we are given a triple $(\mathfrak{a}, m, \kappa) \in \mathcal{E}$ and an auxiliary ideal $\mathfrak{n}$ satisfying (a1)-(a3). Let $S$ be the set of places of $\mathcal{F}$ dividing $\mathfrak{n}$ and let $K$ be the open-compact subgroup defined in (5.15). We retain the notations in 55.7 Recall that $\psi: \Delta_{\mathfrak{c}} \rightarrow \Delta \hookrightarrow \overline{\mathbf{Q}}^{\times}$is a branch character, $\Psi$ is the $\Lambda$-valued Galois character associated to $\psi$ and $\mathcal{M}_{\text {ord }}(K, \Lambda)$ is the space of $\Lambda$-adic ordinary cusp form. Extending the coefficient ring $\mathcal{O}_{\mathfrak{p}}$ of $\Lambda$, the linear functional $l_{\mathfrak{a}, m, \kappa}$ yields a $\Lambda$-module map $l_{\mathfrak{a}, m, \kappa}: \mathcal{M}_{\text {ord }}(K, \Lambda) \rightarrow \Lambda$ compatible with specializations. The global Hecke algebra $\mathcal{H}^{S}(G, K)$ defined in $\$ 3.9$ commutes with the $U_{p}$-operators, thereby acting on $\mathcal{M}_{\text {ord }}(K, \Lambda)$ naturally. We introduce some Hecke operators as follows. For each $v \notin S \cup S_{p}$, let $w$ be a place of $\mathcal{K}$ above $v$ and let $\varpi$ be a unifomizer of $\mathcal{F}_{v}$. If $v$ is split, we identify $G\left(\mathcal{F}_{v}\right)$ with $\mathrm{GL}_{3}\left(\mathcal{F}_{v}\right) \times \mathcal{F}_{v}^{\times}$ according to the choice of $w$ and let

$$
\widetilde{\alpha}_{i, w}:=\left(\left[\begin{array}{cc}
\mathbf{1}_{3-i} & \\
& \varpi \cdot \mathbf{1}_{i}
\end{array}\right], 1\right), i=1,2,3 .
$$

Write $T_{i, w}$ for the characteristic function of $K_{v} \widetilde{\alpha}_{i, w} K_{v}$. Put

$$
\begin{aligned}
& \lambda_{1, w}^{T}=\left(\Psi^{-c}+\varepsilon^{-1}+\Psi \varepsilon^{-2}\right)\left(\operatorname{Frob}_{w}\right), \\
& \lambda_{2, w}^{T}=\left(\Psi^{-c}+\Psi \varepsilon^{-2}+\Psi^{1-c} \varepsilon^{-1}\right)\left(\operatorname{Frob}_{w}\right), \\
& \lambda_{3, w}^{T}=\Psi^{1-c}\left(\operatorname{Frob}_{w}\right) .
\end{aligned}
$$

If $v$ is inert, let $\widetilde{\beta}_{w}:=\operatorname{diag}\left(\varpi^{-1}, 1, \varpi\right)$ and write $Q_{w}$ for the characteristic function of $K_{v} \widetilde{\beta}_{i, w} K_{v}$. Put

$$
\lambda_{w}^{Q}=q_{v}-1+\left(\varepsilon^{-2} \Psi^{c}+\Psi^{-1}\right)\left(\operatorname{Frob}_{w}\right) .
$$

Let $\mathbf{H}_{v}^{\text {ord }}$ be the $\Lambda$-algebra generated by the image of $\left\{T_{i, w}\right\}_{i=1,2,3}$ if $v$ is split (resp. $Q_{w}$ if $v$ is inert) in $\operatorname{End}_{\Lambda}\left(\mathcal{M}_{\text {ord }}(K, \Lambda)\right)$. Let $\mathbf{h}^{\text {ord }}(K, \Lambda)\left(\operatorname{resp} . \mathbf{H}^{\text {ord }}(K, \Lambda)\right)$ be the $\Lambda$-algebra generated by the image of $\mathbf{H}_{v}^{\text {ord }}$ in $\operatorname{End}_{\Lambda}\left(\mathcal{M}_{\text {ord }}^{0}(K, \Lambda)\right.$ ) (resp. $\operatorname{End}_{\Lambda}\left(\mathcal{M}_{\text {ord }}(K, \Lambda)\right)$ for all $v \notin S \cup S_{p}$. Then $\mathbf{h}^{\text {ord }}(K, \Lambda)$ is a reduced and finite $\Lambda$-algebra. In particular $\mathbf{h}^{\text {ord }}(K, \Lambda)$ is henselian. Let $\mathfrak{X}_{\Lambda}^{c l s}$ be a Zariski-dense subset in $\mathfrak{X}_{\Lambda}$ given by

$$
\mathfrak{X}_{\Lambda}^{c l s}=\left\{\widehat{\epsilon} \in \mathfrak{X}_{\Lambda} \mid \widehat{\epsilon} \circ \pi_{\mathbf{T}} \in \mathfrak{X}_{\mathbf{T} / \mathbf{T}^{g l}}^{c l s}\right\},
$$

where $\mathfrak{X}_{\mathbf{T} / \mathbf{T}^{g l}}^{c l s}$ is the space of classical weights (4.3) and $\pi_{\mathbf{T}}$ is defined in (5.25). For

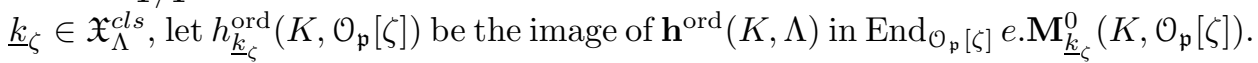
The following proposition is a standard consequence of the control theorems Theorem 4.21 and Cor. 4.23 ( $c f$. SU13, Thm. 6.16]).

Proposition 7.17. For each $\underline{k}_{\zeta} \in \mathfrak{X}_{\Lambda}^{c l s}$, the natural homomorphism

$$
\mathbf{h}^{\text {ord }}(K, \Lambda) \otimes_{\Lambda} \Lambda / P_{\underline{k}_{\zeta}} \rightarrow h_{\underline{k}_{\zeta}}^{\text {ord }}\left(K, \mathcal{O}_{\mathfrak{p}}[\zeta]\right)
$$

has nilpotent kernel.

7.8. Eisenstein ideal. Let $\mathcal{I}_{v}(\Psi)$ be the ideal of $\mathcal{H}_{v}(G, K)$ generated by $\left(T_{i, w}-\right.$ $\left.\lambda_{i, w}^{T}\right)$ if $v$ is split (resp. $\left(Q_{w}-\lambda_{w}^{Q}\right)$ if $v$ is inert) and let $\mathcal{I}^{S}(\Psi)$ be the ideal of $\mathcal{H}^{S}(G, K)$ generated by $\mathcal{I}_{v}(\Psi)$ for all $v \notin S \cup S_{p}$. Let $I^{S}(\Psi)$ be the ideal generated by the image of $\mathcal{I}^{S}(\Psi)$ in $\mathbf{h}^{\text {ord }}(K, \Lambda)$. We call $I^{S}(\Psi)$ the Eisenstein ideal of $\mathbf{h}^{\text {ord }}(K, \Lambda)$. Put

$$
\mathcal{L}^{S}(\Psi)=L_{p}^{S}\left(-1, \Psi_{+} \tau_{\mathcal{K} / \mathcal{F}}\right) L_{p}^{S}(\Psi, \Sigma) \in \Lambda
$$


We now prove the main result in this section.

Theorem 7.18. Suppose that the branch character $\psi$ is primitive outside p, i.e. $\mathfrak{c}$ is the prime-to-p conductor $\mathfrak{c}(\psi)$. We further suppose that the following hypotheses hold:

(1) $p \nmid 3 \cdot h_{\mathcal{K}}^{-} \cdot D_{\mathcal{F}}$,

(2) $\psi$ is unramified at $\Sigma_{p}^{c}$, and $\psi \omega_{\mathcal{K}}^{-a}$ is unramified at $\Sigma_{p}$ for some integer a,

(3) $\mu_{p}(\psi)=0$.

If $\mathcal{L}^{S}(\Psi)$ is not a unit in $\Lambda$, then there exists an ordinary p-adic Eisenstein series $\mathcal{E}=\mathcal{E}^{o r d}(\Psi \mid \mathbf{1}, \mathfrak{n})$ inducing a $\Lambda$-algebra homomorphism

$$
\lambda_{\varepsilon}: \frac{\mathbf{h}^{\text {ord }}(K, \Lambda)}{I^{S}(\Psi)} \longrightarrow \frac{\Lambda}{\left(\mathcal{L}^{S}(\Psi)\right)} .
$$

Proof. The assumption (2) assures us that there exists a point $\widehat{\epsilon} \in \mathfrak{X}_{\Lambda}^{+}$such that $\psi \widehat{\epsilon}$ is the $p$-adic avatar of the Hecke character $\chi=\psi \epsilon$ which is unramified at all places above $p$ (so the infinity type of $\chi$ is $k \Sigma$ and $k \equiv a(\bmod p-1))$. By the choice of $\chi$ and the primitiveness of $\psi$, the conductor $\mathfrak{c}(\chi)$ of $\chi$ is $\mathfrak{c}$ and $\mu_{p}(\chi)=\mu_{p}(\psi)=0$. Thus $\chi$ satisfies Hypothesis 7.12 by Prop. 7.16, It follows from Prop. 7.13 and (5.24) that there exists a triple $(\mathfrak{a}, m, \kappa) \in \mathcal{E}$ and a sufficiently deep auxiliary ideal $\mathfrak{n}$ such that

$$
l_{\mathfrak{a}, m, \kappa}\left(\mathcal{E}^{\text {ord }}(\Psi \mid \mathbf{1}, \mathfrak{n})\right) \equiv l_{\mathfrak{a}, m, \kappa}\left(E^{\text {ord }}(\chi \mid \mathbf{1}, \mathfrak{n})\right) \not \equiv 0\left(\bmod \mathfrak{m}_{p}\right) .
$$

Now we apply Hida theory for $U(2,1)_{/ \mathcal{F}}$ developed in $\$ 4$ Specializing the fundamental exact sequence in Theorem 4.26 via $\pi_{\mathbf{T}}^{\psi}: \widetilde{\Lambda} \rightarrow \Lambda$ defined in (5.25), we obtain the exact sequence

$$
0 \longrightarrow \mathcal{M}_{\text {ord }}^{0}(K, \Lambda) \longrightarrow \mathcal{M}_{\text {ord }}(K, \Lambda) \stackrel{\widehat{\Phi}^{w_{2}}}{\longrightarrow} \bigoplus_{g \in C(K)} \mathcal{M}_{\text {ord }}\left(K_{P}^{g}, \widetilde{\Lambda}\right) \otimes_{\widetilde{\Lambda}} \Lambda \longrightarrow 0 .
$$

Combining the calculation of the constant terms of $\mathcal{E}^{\text {ord }}(\Psi \mid \mathbf{1}, \mathfrak{n})$ in Prop. 6.6, we deduce that there exists a $\Lambda$-adic modular form $\mathcal{E}^{\prime}$ such that

$$
\mathcal{F}:=\mathcal{E}^{\text {ord }}(\Psi \mid \mathbf{1}, \mathfrak{n})-\mathcal{E}^{\prime} \cdot \mathcal{L}^{S}(\Psi) \in \mathcal{M}_{\text {ord }}^{0}(K, \Lambda)
$$

is a $\Lambda$-adic cusp form. Since $\mathcal{L}^{S}(\Psi)$ is not a unit, we have

$$
l_{\mathfrak{a}, m, \kappa}(\mathcal{F}) \equiv l_{\mathfrak{a}, m, \kappa}\left(\mathcal{E}^{\text {ord }}(\Psi \mid \mathbf{1}, \mathfrak{n})\right) \not \equiv 0\left(\bmod \mathfrak{m}_{\Lambda}\right),
$$

and hence $l_{\mathfrak{a}, m, \kappa}(\mathcal{F})$ is a $\Lambda$-adic unit. Define a $\Lambda$-linear functional $\lambda_{\varepsilon}: \mathbf{h}^{\text {ord }}(K, \Lambda) \rightarrow$ $\Lambda$ by

$$
\lambda_{\mathcal{E}}(h)=\frac{l_{\mathfrak{a}, m, \kappa}(h . \mathcal{F})}{l_{\mathfrak{a}, m, \kappa}(\mathcal{F})} \in \Lambda .
$$

A direct computation shows that $\mathcal{I}^{S}(\Psi)$ annihilates $\mathcal{E}^{\text {ord }}(\Psi \mid \mathbf{1}, \mathfrak{n})$ (cf. MS07, Lemma 3.1]). Hence, $\lambda_{\varepsilon}$ induces a surjective $\Lambda$-algebra homomorphism

$$
\lambda_{\varepsilon}: \frac{\mathbf{h}^{\text {ord }}(K, \Lambda)}{I^{S}(\Psi)} \longrightarrow \frac{\Lambda}{\left(\mathcal{L}^{S}(\Psi)\right)} .
$$

This completes the proof. 
Definition 7.19 (The ideal of Eisenstein congruence). Suppose that $\left(\mathcal{L}^{S}(\Psi)\right) \neq \Lambda$. Let $\mathfrak{m}_{\mathcal{E}}$ be the maximal ideal of $\mathbf{h}^{\text {ord }}(K, \Lambda)$ which contains $I^{S}(\Psi)$ in $\mathbf{h}^{\text {ord }}(K, \Lambda)$. Let $\mathbf{h}_{\mathcal{E}}^{\text {ord }}:=\mathbf{h}^{\text {ord }}(K, \Lambda)_{\mathfrak{m}_{\varepsilon}}$ be the local component of $\mathbf{h}^{\text {ord }}(K, \Lambda)$ through which $\lambda_{\mathcal{E}}$ factors. Then $\mathbf{h}_{\mathcal{E}}^{\text {ord }}$ is a local $\Lambda$-algebra which is finite, reduced and $\Lambda$-torsion free. We still denote by $I^{S}(\Psi)$ the ideal generated by the image of $I^{S}(\Psi)$ in $\mathbf{h}_{\mathcal{E}}^{\text {ord }}$. Let $i: \Lambda \rightarrow \mathbf{h}_{\mathcal{\varepsilon}}^{\text {ord }}$ be the structure morphism. Define the ideal of Eisenstein congruence $\operatorname{Eis}(\Psi, S)$ in $\Lambda$ by

$$
\operatorname{Eis}(\Psi, S):=i^{-1}\left(I^{S}(\Psi)\right)=\Lambda \cap I^{S}(\Psi) .
$$

It follows by definition that

$$
\Lambda / \operatorname{Eis}(\Psi, S) \stackrel{\sim}{\rightarrow} \mathbf{h}_{\mathcal{E}}^{\text {ord }} / I^{S}(\Psi) .
$$

The following corollary is an immediate consequence of Theorem 7.18

Corollary 7.20. With the assumptions in Theorem 7.18, we have

$$
\operatorname{Eis}(\Psi, S) \subset\left(L_{p}^{S}\left(-1, \Psi_{+} \tau_{\mathcal{K} / \mathcal{F}}\right) \cdot L_{p}^{S}(\Psi, \Sigma)\right) .
$$

\section{Application to the main CONJECture For CM Fields}

8.1. F. Mainardi in Mai08 proves a divisibility relation between the Eisenstein ideal in the topological ordinary cuspidal Hecke algebra attached to the unitary group $U(2,1)$ and the characteristic power series of an Iwasawa module related to the main conjecture for CM fields. The idea of using lattice construction in his proof is due to Ribet, Wiles and Urban ([Rib76], Wil90 and Urb01]). In this section, we deduce the second inequality $(\mathrm{E} \mid \mathrm{S})$ in the introduction following these ideas. We will work with ordinary cuspidal Hecke algebras for the coherent cohomology. Indeed, since the coherent Hecke algebra is a quotient of the topological Hecke algebra, the Eisenstein ideal defined by Mainardi (Definition 3.4.1 loc. cit.) specializes to our Eisenstein ideal $I^{S}(\Psi)$. However, the divisibility result proved in Mai08 excludes several height one primes of the topological Hecke algebra, so it is not clear to the author if a partial result for the divisibility $(\mathrm{E} \mid \mathrm{S})$ in our setting can be obtained by taking the specialization of Mainardi's divisibility. Therefore, in 88.3 and 88.4 , we elaborate the ideas of Mainardi's work in our framework, making use of theory of the deformation of pseudo-characters developed in the book [BC09, Chaper 1]. The main theorem is Theorem 8.14, and some arithmetic applications of the main theorem are given in 8.6 .

Throughout this section, we let $\mathfrak{n}$ and $S$ be as in Theorem 7.18 and retain the hypotheses (1-2) therein. Denote by $\mathcal{K}_{\infty}^{+}$the cyclotomic $\mathbf{Z}_{p}$-extension and by $\mathcal{K}_{\infty}^{-}$the anticyclotomic $\mathbf{Z}_{p}^{d}$-extension of $\mathcal{K}$. Let $\Gamma_{\mathcal{K}}^{+}=\operatorname{Gal}\left(\mathcal{K}_{\infty}^{+} / \mathcal{K}\right)\left(\operatorname{resp} . \Gamma_{\mathcal{K}}^{-}=\right.$ $\left.\operatorname{Gal}\left(\mathcal{K}_{\infty}^{-} / \mathcal{K}\right)\right)$ and let $\Lambda_{+}=\mathcal{O}_{\mathfrak{p}} \llbracket \Gamma_{\mathcal{K}}^{+} \rrbracket\left(\right.$ resp. $\left.\Lambda_{-}=\mathcal{O}_{\mathfrak{p}} \llbracket \Gamma_{\mathcal{K}}^{-} \rrbracket\right)$. We have a canonical isomorphism $\operatorname{Gal}\left(\mathcal{K}_{\infty} / \mathcal{K}\right)=\Gamma_{\mathcal{K}}^{+} \times \Gamma_{\mathcal{K}}^{-}$. Thus $\Lambda_{+}$and $\Lambda_{-}$can be regarded as subrings of $\Lambda=\mathcal{O}_{\mathfrak{p}} \llbracket \operatorname{Gal}\left(\mathcal{K}_{\infty} / \mathcal{K}\right) \rrbracket$. We fix a set of the generators $\left\{\gamma_{-}^{(i)}\right\}_{i=1, \ldots, d}\left(\right.$ resp. $\left.\gamma_{+}\right)$of $\Gamma_{\mathcal{K}}^{-}$(resp. $\Gamma_{\mathcal{K}}^{+}$). Then $\Lambda=\mathcal{O}_{\mathfrak{p}} \llbracket T_{+}, S_{1}, \ldots, S_{d} \rrbracket=\Lambda_{+} \otimes_{\mathcal{O}_{\mathfrak{p}}} \Lambda_{-}$is the $(d+1)$-variable formal power series ring over $\mathcal{O}_{\mathfrak{p}}$ with the cyclotomic variable $T_{+}=\gamma_{+}-1$ and the anticyclotomic variables $\left\{S_{i}=\gamma_{-}^{(i)}-1\right\}_{i=1, \ldots, d}$. 


\subsection{Selmer groups for CM fields.}

8.2.1. Definitions. Let $\mathcal{L} / \mathcal{F}$ be a finite extension. For a subset $T$ of places of $\mathcal{F}$, denote by $T^{\mathcal{L}}$ the set of places of $\mathcal{L}$ above $T$. Denote by $\mathcal{L}_{S}$ the maximal algebraic extension of $\mathcal{L}$ which is unramified outside $S^{\mathcal{L}} \cup S_{p}^{\mathcal{L}}$. Let $A$ be a profinite $\mathbf{Z}_{p}$-algebra and $\rho: \operatorname{Gal}\left(\mathcal{L}_{S} / \mathcal{L}\right) \rightarrow A^{\times}$be an $A$-valued character. For partitions $S_{p}^{\mathcal{L}}=\Sigma_{0} \sqcup \Sigma_{1}$ and $S=S_{0} \sqcup S_{1}$, we define the $S_{0}$-primitive Selmer group for $\left(\rho, \Sigma_{0}\right)$ by

$$
\operatorname{Sel}_{\mathcal{L}}^{S_{0}}\left(\rho, \Sigma_{0}\right)=\operatorname{ker}\left\{H^{1}\left(\mathcal{L}_{S} / \mathcal{L}, \rho \otimes_{A} A^{*}\right) \rightarrow \prod_{v \in S_{1}^{\mathcal{L}} \cup \Sigma_{1}} H^{1}\left(I_{v}, \rho \otimes_{A} A^{*}\right)\right\},
$$

where $A^{*}=\operatorname{Hom}_{\text {cont }}\left(A, \mathbf{Q}_{p} / \mathbf{Z}_{p}\right)$ is the Pontryagin dual of $A$ and $I_{v}$ are respective inertia subgroups. We denote by $X_{\mathcal{L}}^{S_{0}}\left(\rho, \Sigma_{0}\right)$ the Pontryagin dual of $\operatorname{Sel}_{\mathcal{L}}^{S_{0}}\left(\rho, \Sigma_{0}\right)$. When $S_{0}$ or $\Sigma_{0}$ is the empty set (so $S_{1}=S$ or $\Sigma_{1}=S_{p}^{\mathcal{L}}$ is the set of $p$-adic places of $\mathcal{L}$ ), we drop it from the notation.

We define Selmer groups for the $\Lambda$-valued character $\Psi$ attached to the branch character $\psi$ and the $p$-ordinary CM-type $\Sigma$. Recall that $\Sigma$ gives rise to a partition $S_{p}^{\mathcal{K}}=\Sigma_{p} \sqcup \Sigma_{p}^{c}$ (1.4). Let $\Psi^{D}$ be the Cartier dual of $\Psi$, namely, $\Psi^{D}: \operatorname{Gal}\left(\mathcal{K}_{S} / \mathcal{K}\right) \rightarrow$ $\Lambda^{\times}$is the character defined by $\Psi^{D}(g)=\Psi^{-1} \varepsilon(g)$. Let $\operatorname{Sel}_{\mathcal{K}}^{S_{0}}(\Psi, \Sigma)=\operatorname{Sel}_{\mathcal{K}}^{S_{0}}\left(\Psi, \Sigma_{p}\right)$ $\left(\operatorname{resp} . \operatorname{Sel}_{\mathcal{K}}^{S_{0}}\left(\Psi^{D}, \Sigma^{c}\right)=\operatorname{Sel}_{\mathcal{K}}^{S_{0}}\left(\Psi^{D}, \Sigma_{p}^{c}\right)\right)$ and let $X_{\mathcal{K}}^{S_{0}}(\Psi, \Sigma)\left(\operatorname{resp} . X_{\mathcal{K}}^{S_{0}}\left(\Psi^{D}, \Sigma^{c}\right)\right)$ be the respective Pontryagin dual.

8.2.2. Let $\mathcal{R}$ be a commutative Iwasawa algebra over $\mathcal{O}_{\mathfrak{p}}$. Suppose that $\mathcal{R}$ is a quotient ring of $\Lambda$ with the quotient map $\pi_{\mathcal{R}}: \Lambda \rightarrow \mathcal{R}$. Define the $\mathcal{R}$-adic Selmer group for $(\Psi, \Sigma)$ by

$$
\operatorname{Sel}_{\mathcal{K}}^{S_{0}}(\Psi, \Sigma, \mathcal{R})=\operatorname{Sel}_{\mathcal{K}}^{S_{0}}\left(\pi_{\mathcal{R}} \circ \Psi, \Sigma_{p}\right)
$$

Note that $\operatorname{Sel}_{\mathcal{K}}(\Psi, \Sigma, \mathcal{R})=\operatorname{Sel}_{\mathcal{K}}^{S_{\psi}}(\Psi, \Sigma, \mathcal{R})$. It is well known that $\operatorname{Sel}_{\mathcal{K}}^{S}(\Psi, \Sigma, \mathcal{R})$ is a co-finitely generated discrete $\mathcal{R}$-module. The Pontryagin dual of $\operatorname{Sel}_{\mathcal{K}}^{S}(\Psi, \Sigma, \mathcal{R})$ is denoted by $X_{\mathcal{K}}^{S}(\Psi, \Sigma, \mathcal{R})$ which is a finitely generated compact $\mathcal{R}$-module. We recall the definition of a characteristic ideal of a finitely generated $\mathcal{R}$-module.

Definition 8.1. Let $h_{1}(\mathcal{R})$ be the set of height one primes of $\mathcal{R}$. If $X$ is a finitely generated compact $\mathcal{R}$-module, then for each $P \in \mathrm{ht}_{1}(\mathcal{R})$ we define

$$
\ell_{P}(X)=\operatorname{length}_{\mathcal{R}_{P}}\left(X \otimes_{\mathcal{R}} \mathcal{R}_{P}\right) .
$$

If $X$ is $\mathcal{R}$-torsion, then we define the characteristic ideal $\operatorname{char}_{\mathcal{R}} X$ of $X$ by

$$
\operatorname{char}_{\mathcal{R}} X:=\prod_{P \in \mathrm{ht}_{1} \mathcal{R}} P^{\ell_{P}(X)} .
$$

If $X$ has positive $\mathcal{R}$-rank, we put $\operatorname{char}_{\mathcal{R}} X=0$. Define the characteristic power series $F_{\mathcal{R}}(X) \in \mathcal{R}$ to be a generator of $\operatorname{char}_{\mathcal{R}} X$ (unique up to a unit). Denote by $F_{\Lambda}(\Psi, \Sigma)\left(\right.$ resp. $\left.F_{\Lambda}\left(\Psi^{D}, \Sigma^{c}\right)\right)$ the characteristic power series of $X_{\mathcal{K}}(\Psi, \Sigma)$ (resp. $\left.X_{\mathcal{K}}\left(\Psi^{D}, \Sigma^{c}\right)\right)$.

If $\mathcal{S}$ is a co-finitely generated discrete $\mathcal{R}$-module, we define $\ell_{P}(\mathcal{S})=\ell_{P}\left(\mathcal{S}^{*}\right)$ and $\operatorname{char}_{\mathcal{R}} \mathcal{S}=\operatorname{char}_{\mathcal{R}} \mathcal{S}^{*}$.

Lemma 8.2. Let $X$ be a finitely generated $\mathcal{R}$-module and $\mathcal{L}$ be a non-zero element in $\mathcal{R}$. The following statements are equivalent.

(1) $\ell_{P}(X) \geq \operatorname{ord}_{P}(\mathcal{L})$ for all $P \in \mathrm{ht}_{1}(\mathcal{R})$. 
(2) $\operatorname{Fitt}_{\mathcal{R}}(X) \subset(\mathcal{L})$.

(3) $\operatorname{char}_{\mathcal{R}} X \subset(\mathcal{L})$

Proof. Since $\mathcal{R}$ is isomorphic to a formal power series over $\mathcal{O}_{\mathfrak{p}}, \mathcal{R}$ is a unique factorization domain. Thus, each height one prime $P \in \mathrm{ht}_{1}(\mathcal{R})$ is principal and $\mathcal{R}_{P}$ is a principal ideal domain. This implies (1) and (3) are equivalent. The equivalence between (1) and (2) follows from the base change property of Fitting ideals $\operatorname{Fitt}_{\mathcal{R}}(X)_{P}=\operatorname{Fitt}_{\mathcal{R}_{P}}\left(X_{P}\right)=P^{\ell_{P}(X)}$.

For each place $w$ of $\mathcal{K}$, we fix a local decomposition group $D_{w}$ of $G_{\mathcal{K}}$ and let $I_{w}$ be the inertia subgroup of $D_{w}$. The following observation is useful in studying the specialization of the characteristic ideal of the Selmer groups.

Lemma 8.3. Let $f \in \mathcal{R}$ be a non-zero divisor. If the kernel and cokernel of the restriction map

$$
H^{0}\left(\mathcal{K}_{S} / \mathcal{K}, \Psi \otimes_{\Lambda} \mathcal{R}^{*}\right) \otimes \mathcal{R} /(f) \rightarrow \prod_{w \in S^{\mathcal{K}} \cup \Sigma_{p}^{c}}\left(H^{0}\left(I_{w}, \Psi \otimes_{\Lambda} \mathcal{R}^{*}\right) \otimes \mathcal{R} /(f)\right)^{D_{w}}
$$

are pseudo-null $\mathcal{R} /(f)$-modules, then we have a natural $\mathcal{R} /(f)$-module pseudoisomorphism

$$
\operatorname{Sel}_{\mathcal{K}}(\Psi, \Sigma, \mathcal{R} /(f)) \sim \operatorname{Sel}_{\mathcal{K}}(\Psi, \Sigma, \mathcal{R})[f] .
$$

Proof. Put $S(\mathcal{R})=\operatorname{Sel}_{\mathcal{K}}(\Psi, \Sigma, \mathcal{R})$ and $B=\Psi \otimes_{\Lambda} \mathcal{R}^{*}$. It follows from the following long exact sequence together with the snake lemma

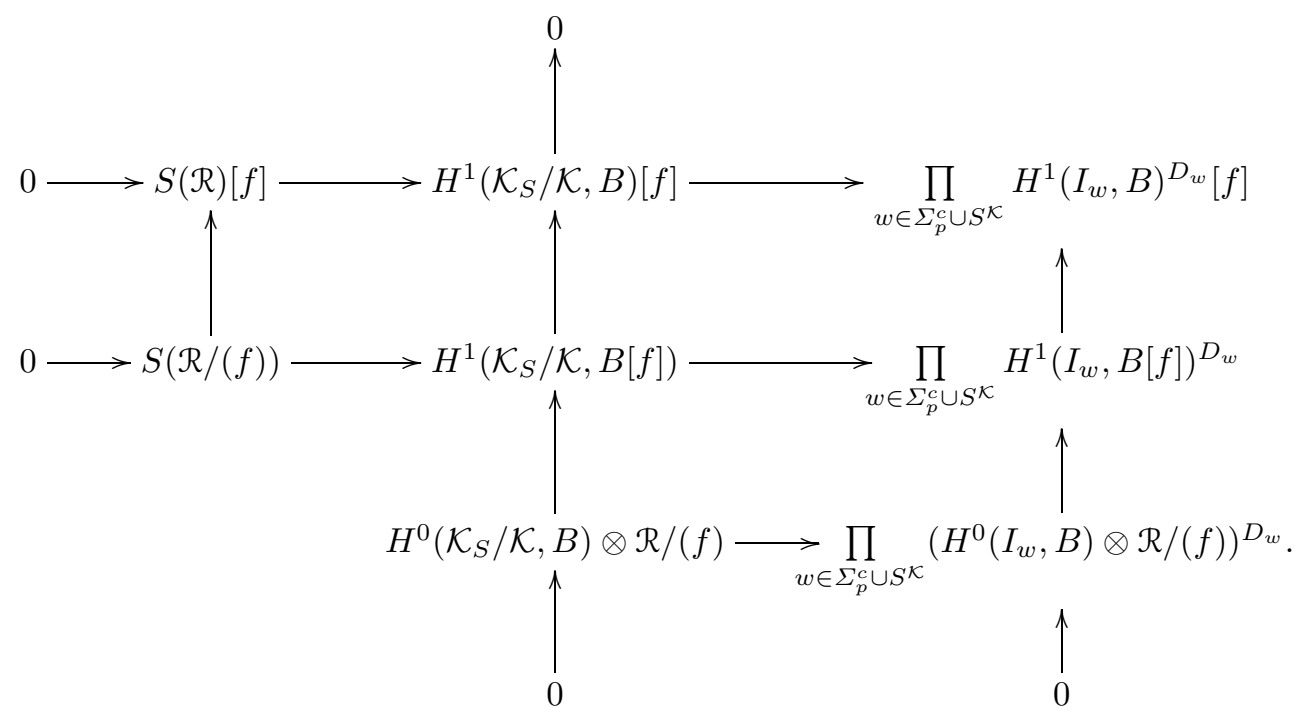

Remark 8.4 (Specializations). Let $t$ be a positive integer such that $d+1-t \geq 2$.

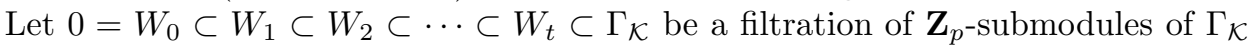
so that for $i=1, \ldots, t$, the quotient $W_{i} / W_{i+1} \simeq \mathbf{Z}_{p}$ and $\Gamma_{\mathcal{K}} / W_{t}$ is a free $\mathbf{Z}_{p^{-} \text {-module }}$ of rank $d+1-t$. Let $L_{i}=\mathcal{K}_{\infty}^{W_{i}}$ and $\Lambda_{i}=\Lambda\left(L_{i}\right)$. Then $L_{i}$ is a $\mathbf{Z}_{p}^{d-i+1}$-extension of $\mathcal{K}$ and the restriction map $\Gamma_{\mathcal{K}} / W_{i-1} \rightarrow \Gamma_{\mathcal{K}} / W_{i}$ induces a surjective morphism $\Lambda_{i-1} \rightarrow \Lambda_{i}$. The kernel of the map $\Lambda_{i-1} \rightarrow \Lambda_{i}$ is generated by an element $a_{i} \in \Lambda$ such that $p \nmid a_{i}$. 
For $1 \leq i \leq t$, we put

$$
\begin{aligned}
N^{i} & =H^{0}\left(\mathcal{K}_{S} / \mathcal{K}, \Psi \otimes \Lambda_{i-1}^{*}\right) \otimes_{\Lambda_{i-1}} \Lambda_{i}, \\
N_{w}^{i} & =\left(H^{0}\left(I_{w}, \Psi \otimes \Lambda_{i-1}^{*}\right) \otimes_{\Lambda_{i-1}} \Lambda_{i}\right)^{D_{w}}, w \in \Sigma_{p}^{c} \cup S^{\mathcal{K}} .
\end{aligned}
$$

The Pontryagin dual of $N^{i}$ is $\left.\mathcal{O}_{\mathfrak{p}} /\left(\psi\left(\Delta_{\mathfrak{c}}\right)-1\right)\right)$, which is a pseudo-null $\Lambda_{i}$-module as $d+1-i \geq 2$. If $w \mid \mathfrak{c}(\psi)$, the $\Lambda$-ideal $\left(\Psi\left(I_{w}\right)-1\right)=\left(\psi\left(I_{w}\right)-1\right)=\left(p^{m}\right)$ for some $m \geq 0$. Since $\left(\Lambda_{i-1} / p^{m} \Lambda_{i-1}\right)^{*}$ is $a_{i}$ divisible, $N_{w}^{i}=0$. For $w \in S_{p}^{\mathcal{K}}$, let $\mathfrak{I}_{w}$ be the ideal of $\Lambda$ generated by $\{\Psi(g)-1\}_{g \in D_{w}}$. Consider the following two cases:

(i) $d \geq 2, t=1$ and $W_{1}=\Gamma_{\mathcal{K}}^{+}$: In this case, $\Lambda_{1}=\Lambda /\left(T_{+}\right)$. Then it is shown in [HT94, Prop.3.2.3] that the image of $\mathfrak{I}_{w}$ in $\Lambda /\left(T_{+}\right)$has a height of at least two.

(ii) $W_{t} \subset \Gamma_{-}$: Since $\mathfrak{I}_{w}$ contains $\left(1+T_{+}\right)^{p^{m_{0}}}\left(1+S_{j_{0}}\right)-1$ and $\Psi\left(\right.$ Frob $\left._{w}\right)=$ $\psi\left(\varpi_{w}\right) \prod_{j=1}^{d}\left(1+S_{j}\right)^{b_{j}}-1$ for some $1 \leq j_{0} \leq d$ and $m_{0}, b_{j} \in \mathbf{Z}$, the image of $\mathfrak{I}_{w}$ in $\Lambda_{i}$ for all $i$ has a height of at least two.

In either of the above cases, by Lemma 8.3 we have a natural $\Lambda_{t}$-module pseudoisomorphism:

$$
\operatorname{Sel}_{\mathcal{K}}\left(\Psi, \Sigma, \Lambda_{t}\right) \sim \operatorname{Sel}_{\mathcal{K}}(\Psi, \Sigma, \Lambda)\left[a_{1}, \ldots, a_{t}\right] .
$$

8.2.3. For a Galois extension $L \subset \mathcal{K}_{\infty}$ of $\mathcal{K}$, we let $X_{\Sigma}(L)$ be the maximal $\Sigma_{p^{-}}$ ramified $p$-extension of $L$ and $X_{\Sigma}^{(\psi)}(L)$ be its $\psi$-isotypic quotient. Let $\Lambda(L):=$ $\mathbf{Z}_{p}[\psi] \llbracket \operatorname{Gal}(L / \mathcal{K}) \rrbracket$. If $p \nmid \sharp(\Delta)$, it follows from the Serre-Hochschild exact sequence that we have a natural $\Lambda(L)$-module isomorphism

$$
\text { res : } X_{\mathcal{K}}(\Psi, \Sigma, \Lambda(L)) \stackrel{\sim}{\rightarrow} X_{\Sigma}^{(\psi)}(L) \otimes_{\mathbf{z}_{p}[\psi]} \mathcal{O}_{\mathfrak{p}} .
$$

When $L=\mathcal{K}_{\infty}$, let $F_{\psi, \Sigma}=F_{\Lambda\left(\mathcal{K}_{\infty}\right)}\left(X_{\Sigma}\left(\mathcal{K}_{\infty}\right)\right)$. In particular, we have $F_{\psi, \Sigma} \cdot \Lambda=$ $\left(F_{\Lambda}(\Psi, \Sigma)\right)$.

8.3. Pseudo-representation and the lattice construction. We introduced in \$7.8 the Eisenstein ideal $I^{S}(\Psi)$ and the associated local component $\left(\mathbf{h}_{\mathcal{\varepsilon}}^{\text {ord }}, \mathfrak{m}_{\mathcal{\varepsilon}}\right)$ of $\mathbf{h}^{\text {ord }}(K, \Lambda)$. For brevity, in what follows we write $(R, Q)=\left(\mathbf{h}_{\mathcal{E}}^{\text {ord }}, \mathfrak{m}_{\mathcal{E}}\right), \mathcal{I}=I^{S}(\Psi)$, $G_{\mathcal{K}, S}=\operatorname{Gal}\left(\mathcal{K}_{S} / \mathcal{K}\right)$ and

$$
\delta_{1}=\Psi^{-c} \varepsilon, \delta_{2}=\mathbf{1}, \delta_{3}=\Psi \varepsilon^{-1}
$$

(recall that $\varepsilon: G_{\mathcal{K}, S} \rightarrow \mathbf{Z}_{p}^{\times}$is the $p$-adic cyclotomic character). Let $\mathfrak{X}_{R}^{c l s}$ be the subset of arithmetic points in $\mathfrak{X}_{R}:=\operatorname{Hom}_{\text {cont }}\left(R, \mathbf{C}_{p}\right) \subset \operatorname{Spec} R\left(\mathbf{C}_{p}\right)$ such that to each $x \in \mathfrak{X}_{R}^{c l s}$ we can attach a $p$-ordinary cuspidal holomorphic automorphic representation $\pi_{x}$ with the ring homomorphism $\boldsymbol{\lambda}_{\pi_{x}}: R \rightarrow \mathbf{C}_{p}$. We shall call $\mathfrak{X}_{R}^{c l s}$ the space of classical weights. Thanks to the work of Rogawski Rog92 and the techniques in BR93, it is known that there exists a continuous $p$-adic semi-simple Galois representation $R_{p}\left(\pi_{x}\right): G \rightarrow \mathrm{GL}_{3}\left(\mathbf{C}_{p}\right)$ attached to $\pi_{x}$ for each $x \in \mathfrak{X}_{R}^{c l s}$ such that $\rho_{x}:=R_{p}\left(\pi_{x}\right)(1)$ is conjugate self-dual (i.e. $\rho_{x}^{\vee} \simeq \rho_{x}^{c}$, where $c$ is the complex conjugation) and $\operatorname{Tr} R_{p}\left(\pi_{x}\right)\left(\operatorname{Frob}_{w}\right)=\boldsymbol{\lambda}_{\pi_{x}}\left(T_{1, w}\right)$ for each split place $w \notin$ $S^{\mathcal{K}}$, where $T_{1, w}$ are the Hecke operators defined in $\$ 7.7$ ( $c f$. [Mai08, Thm. 4.1.1] and [BC04, §3.2]).

Let $i_{R, \Lambda}: \operatorname{Spec} R\left(\mathbf{C}_{p}\right) \rightarrow \operatorname{Spec} \Lambda\left(\mathbf{C}_{p}\right)$ be the structure morphism. It is clear that $\mathfrak{X}_{R}^{c l s}$ contains $i_{R, \Lambda}^{-1}\left(\mathfrak{X}_{\Lambda}^{c l s}\right)$, and hence $\mathfrak{X}_{R}^{\text {cls }}$ is also Zariski dense in $\mathfrak{X}_{R}$. Therefore, by a standard patching argument in the theory of pseudo-character, we can glue trace functions $\operatorname{Tr} \rho_{x}: G_{\mathcal{K}, S} \rightarrow \mathbf{C}_{p}$ for all $x \in \mathfrak{X}_{R}^{c l s}$ and obtain a unique pseudo-character 
$\mathcal{T}: G_{\mathcal{K}, S} \rightarrow R$ such that $\mathcal{T}\left(\right.$ Frob $\left._{w}\right)=T_{1, w} \cdot \varepsilon\left(\right.$ Frob $\left._{w}\right)$ for split places $w \notin S^{\mathcal{K}} \cup S_{p}^{\mathcal{K}}$ (cf. [SU13, Prop. 7.3]). By the very definition of $\mathcal{I}=I^{S}(\Psi)$ in 7 7.8, we have

$$
\mathcal{T}\left(\operatorname{Frob}_{w}\right)=T_{1, w} \cdot \varepsilon\left(\operatorname{Frob}_{w}\right) \equiv \lambda_{1, w}^{T}=\left(\varepsilon \Psi^{-c}+\mathbf{1}+\Psi \varepsilon^{-1}\right)\left(\operatorname{Frob}_{w}\right)(\bmod \mathcal{I})
$$

for all but finitely Frob $_{w}$ at primes $w$ split in $\mathcal{K} / \mathcal{F}$, and hence by Chebotarev's density theorem,

$$
\mathcal{T} \equiv \delta_{1}+\delta_{2}+\delta_{3}(\bmod \mathcal{I}) .
$$

We further assume the following hypothesis holds

$$
\psi^{1+c} \not \equiv \omega_{\mathcal{K}}^{2}(\bmod \mathfrak{m}) \text {. }
$$

Thus $\mathcal{T}$ is a residually multiplicity free pseudo-character, i.e. the residual characters $\delta_{i}\left(\bmod \mathfrak{m}_{\Lambda}\right)$ are pairwise distinct.

We shall construct an $R\left[G_{\mathcal{K}, S}\right]$-module $\mathscr{L}$ with certain good properties. This is the so-called lattice construction due to E. Urban who generalizes Wiles' construction of cocycles in [Wi190]. (See [Urb99, Urb01] and [SU13, §4].) Here we use the language of generalized matrix algebra (GMA) in Bellaïche and Chenvier's book BC09, Chapter 1]. Recall that a GMA datum $\mathcal{D}=\left\{e_{i}, \varrho_{i}\right\}_{i=1,2,3}$ for $\left(\mathcal{T}, \delta_{i}\right)$ consists of orthogonal idempotents $e_{i}$ together with isomorphisms $\varrho_{i}: e_{i} R\left[G_{\mathcal{K}, S}\right] e_{i} \stackrel{\sim}{\rightarrow} R$ such that $\varrho_{i} \otimes R / \mathcal{I}=\delta_{i}$ ([BC09, Def.1.3.1]). Let $F=$ Frac $R$ be the totally fractional field of $R$. By [BC09, Thm. 1.4.4], we can associate a generalized matrix algebra with $\mathcal{D}$. To be precise, there exists a three-dimensional $F$-vector space $\mathscr{V}$ with a basis $\left\{v_{i}\right\}_{i=1,2,3}$ and a genuine representation $\boldsymbol{\rho}: R\left[G_{\mathcal{K}, S}\right] \rightarrow \operatorname{End}_{F}(\mathscr{V})$ such that according to the basis $\left\{v_{i}\right\}_{i=1,2,3}, \boldsymbol{\rho}\left(R\left[G_{\mathcal{K}, S}\right]\right)$ is isomorphic to the generalized matrix algebra

$$
\boldsymbol{\rho}\left(R\left[G_{\mathcal{K}, S}\right]\right)=\left[\begin{array}{ccc}
R & R_{12} & R_{13} \\
R_{21} & R & R_{23} \\
R_{31} & R_{32} & R
\end{array}\right],
$$

where $R_{i j}$ are fractional ideals in $F$ such that $R_{i j} R_{j i} \subset \mathcal{I}$ for $i \neq j$. For $g \in R\left[G_{\mathcal{K}, S}\right]$, we write $\boldsymbol{\rho}(g)$ as

$$
\boldsymbol{\rho}(g)=\left[\begin{array}{lll}
a_{11}(g) & a_{12}(g) & a_{13}(g) \\
a_{21}(g) & a_{22}(g) & a_{23}(g) \\
a_{31}(g) & a_{32}(g) & a_{33}(g)
\end{array}\right], a_{i j}(g) \in R_{i j} .
$$

Moreover, in virtue of the conjugate self-duality of Galois representations, we have

$$
\mathcal{T}\left(g^{-c}\right)=\mathcal{T}(g), g^{-c}:=c g^{-1} c .
$$

According to [BC09, Lemma 1.8.3] we can manage $\boldsymbol{\rho}$ such that

$$
\boldsymbol{\rho}^{\perp}(g):=\boldsymbol{\rho}\left(g^{-c}\right)^{t}=w \boldsymbol{\rho}(g) w^{-1}, \quad w=\left[\begin{array}{lll} 
& & 1 \\
& 1 &
\end{array}\right] .
$$

Then (8.3) implies

$$
a_{12}\left(g^{-c}\right)=a_{23}(g) \text { and } R_{12}=R_{23} .
$$

Let $\mathscr{L}_{3}:=R v_{3}$ and let $\mathscr{L}=R\left[G_{\mathcal{K}, S}\right] v_{3}$ be the $R\left[G_{\mathcal{K}, S}\right]$-module generated by $\mathscr{L}_{3}$. By definition, $\mathscr{L}$ has the following decomposition as $R$-modules:

$$
\mathscr{L}=\mathscr{D} \oplus \mathscr{L}_{3}, \mathscr{D}=R_{13} v_{1} \oplus R_{23} v_{2} .
$$


By the relation $R_{i j} R_{j i} \subset \mathcal{I}$, we have the following exact sequence as $R\left[G_{\mathcal{K}, S}\right]$ modules:

$$
0 \longrightarrow \mathscr{D} \otimes R / \mathcal{I} \longrightarrow \mathscr{L} \otimes R / \mathcal{I} \longrightarrow \delta_{3} \otimes R / \mathcal{I} \longrightarrow 0 .
$$

Lemma 8.5. Every $R\left[G_{\mathcal{K}, S}\right]$-module quotient of $\mathscr{L} / Q \mathscr{L} \otimes \delta_{3}^{-1}$ is an $R / Q$-module with the trivial $G$-action.

Proof. Suppose $\mathscr{L}$ has an irreducible quotient $\mathscr{L} / \mathscr{N} \stackrel{\sim}{\rightarrow} \tau$ with $\tau \delta_{3}^{-1} \nsucceq 1$. Put $\mathscr{L}^{\prime}=\mathscr{L} /(\mathscr{N}+\mathscr{D}+Q \mathscr{L})$. Then we have $\delta_{3} \otimes R / Q \rightarrow \mathscr{L}^{\prime}$ and $\tau \longrightarrow \mathscr{L}^{\prime}$. This implies $\mathscr{L}^{\prime}=0$ and thereby $\mathscr{L}=\mathscr{N}+\mathscr{D}+Q \mathscr{L}$. By the existence of idempotent $e_{3} \in \boldsymbol{\rho}\left(R\left[G_{\mathcal{K}, S}\right]\right)$ such that $e_{3} v_{3}=v_{3}$ and $e_{3} v_{i}=0$ for $i \neq 3$, we deduce that $v_{3} \in$ $\mathscr{N}$. Because $\mathscr{N}$ is an $R\left[G_{\mathcal{K}, S}\right]$-module and $v_{3}$ generates $\mathscr{L} \otimes k$ as an $R / Q\left[G_{\mathcal{K}, S}\right]$ module, $\mathscr{N}+Q \mathscr{L}=\mathscr{L}$. Hence by the Nakayama lemma $\mathscr{N}=\mathscr{L}$, which is a contradiction.

We set

$$
\begin{aligned}
& \mathscr{I}_{1}=R_{13}, \mathscr{J}_{1}=R_{12} R_{23}+I R_{13} ; \\
& \mathscr{I}_{2}=R_{23}, \mathscr{J}_{2}=R_{21} R_{13}+I R_{23} .
\end{aligned}
$$

For $\{i, j\}=\{1,2\}$, let $\mathscr{M}_{i}=I \mathscr{L}+\mathscr{J}_{i} v_{i}+R_{j 3} v_{j}$ be a sub- $R$-module in $\mathscr{L}$. It can be verified easily that $\mathscr{M}_{i}$ is $G$-stable and $\mathscr{L} / \mathscr{M}_{i}$ fits in the following exact sequence of $R\left[G_{\mathcal{K}, S}\right]$-modules:

$$
0 \longrightarrow \mathscr{I}_{i} / \mathscr{J}_{i} \otimes \mathbf{1} \longrightarrow \mathscr{L} / \mathscr{M}_{i} \otimes \delta_{i}^{-1} \longrightarrow R / \mathcal{I} \otimes \delta_{3} \delta_{i}^{-1} \longrightarrow 0,
$$

where 1 is the trivial $G_{\mathcal{K}, S}$-action. The Pontryagin dual of (8.7) induces the exact sequence

$$
\begin{array}{r}
H^{0}\left(G_{\mathcal{K}, S},\left(\mathscr{L} / \mathscr{M}_{i}\right)^{*}\right) \longrightarrow H^{0}\left(G_{\mathcal{K}, S},\left(\mathscr{I}_{i} / \mathscr{J}_{i}\right)^{*}\right) \\
\quad=\left(\mathscr{I}_{i} / \mathscr{J}_{i}\right)^{*} \stackrel{\Delta_{i}}{\longrightarrow} H^{1}\left(G_{\mathcal{K}, S},(R / \mathcal{I})^{*} \otimes \delta_{3}^{-1} \delta_{i}\right) .
\end{array}
$$

The natural homomorphism $q:(R / \mathcal{I})^{*} \stackrel{\sim}{\rightarrow}(\Lambda / \operatorname{Eis}(\Psi, S))^{*} \hookrightarrow \Lambda^{*}$ gives rise to a homomorphism

$\overline{\boldsymbol{c}}_{i}:=q_{*} \circ \Delta_{i}:\left(\mathscr{I}_{i} / \mathscr{J}_{i}\right)^{*} \stackrel{\Delta_{i}}{\longrightarrow} H^{1}\left(G_{\mathcal{K}, S},(R / \mathcal{I})^{*} \otimes \delta_{3}^{-1} \delta_{i}\right) \stackrel{q_{*}}{\longrightarrow} H^{1}\left(G_{\mathcal{K}, S}, \Lambda^{*} \otimes \delta_{3}^{-1} \delta_{i}\right)$.

Indeed, for each $f \in\left(\mathscr{I}_{i} / \mathscr{J}_{i}\right)^{*}=\operatorname{Hom}_{\text {cont }}\left(\mathscr{I}_{i} / \mathscr{J}_{i}, \mathbf{Q}_{p} / \mathbf{Z}_{p}\right)$ we define the 1-cocycle $\boldsymbol{c}_{i}(f): G_{\mathcal{K}, S} \rightarrow \Lambda^{*} \otimes \delta_{3}^{-1} \delta_{i}$ by

$$
\boldsymbol{c}_{i}(f)(g)(\lambda)=f\left(\delta_{3}^{-1}(g) a_{i 3}(g) \lambda\right) \quad\left(g \in G_{\mathcal{K}, S}, \lambda \in \Lambda\right) .
$$

Then $\overline{\boldsymbol{c}}_{i}(f)$ is the class $\left[\boldsymbol{c}_{i}(f)\right]$ in $H^{1}\left(G_{\mathcal{K}, S}, \Lambda^{*} \otimes \delta_{3}^{-1} \delta_{i}\right)$.

Lemma 8.6. The homomorphisms $\overline{\boldsymbol{c}}_{i}$ are injective.

Proof. Because $\delta_{3} \not \equiv \delta_{i}\left(\bmod \mathfrak{m}_{\Lambda}\right)$ for $i=1,2$, the map $q_{*}$ is injective. Hence by (8.8) it suffices to show $H^{0}\left(G_{\mathcal{K}, S},\left(\mathscr{L} / \mathscr{M}_{i} \otimes \delta_{i}^{-1}\right)^{*}\right)=0$. By Lemma 8.5, we have

$$
\operatorname{Hom}_{G \mathcal{K}, S}\left(\mathscr{L} \otimes \delta_{i}^{-1}, \mathbf{Q}_{p} / \mathbf{Z}_{p}\right)=0 .
$$

Therefore we have

$$
\begin{aligned}
& H^{0}\left(G_{\mathcal{K}, S},\left(\mathscr{L} / \mathscr{M} \otimes \delta_{1}^{-1}\right)^{*}\right) \\
& \quad=\operatorname{Hom}_{G_{\mathcal{K}, S}}\left(\mathscr{L} / \mathscr{M} \otimes \delta_{1}^{-1}, \mathbf{Q}_{p} / \mathbf{Z}_{p}\right) \hookrightarrow \operatorname{Hom}_{G_{\mathcal{K}, S}}\left(\mathscr{L} \otimes \delta_{i}^{-1}, \mathbf{Q}_{p} / \mathbf{Z}_{p}\right)=0 .
\end{aligned}
$$


8.4. $p$-adic Galois representations. In this subsection, we study local $p$-adic Galois representations associated to ordinary cohomological automorphic forms with separable weights and deduce that the maps $\overline{\boldsymbol{c}}_{i}$ in (8.9) actually factor through the corresponding Selmer groups. Let $\operatorname{rec}_{p}:\left(\mathcal{K} \otimes_{\mathbf{Q}} \mathbf{Q}_{p}\right)^{\times} \simeq D_{p}^{a b}:=\prod_{w \mid p} D_{w}^{a b}$ be the geometrically normalized local reciprocity law. We say a $p$-adic character $\widehat{\chi}: D_{p} \rightarrow \mathbf{C}_{p}^{\times}$is separable if $\widehat{\chi}\left(\operatorname{rec}_{p}\left(z_{p}\right)\right)=z_{p}^{k \Sigma+\underline{a}(1-c)}$ for $z_{p} \in\left(\mathcal{O}_{\mathcal{K}} \otimes \mathbf{Z}_{p}\right)^{\times}$, where $k>2$ is an integer and $\underline{a}=\sum_{\sigma \in \Sigma} a_{\sigma} \sigma \in \mathbf{Z}_{\geq 0}[\Sigma]$ is a $d$-tuple of non-negative integers such that

$$
k+a_{\sigma}-1>a_{\sigma^{\prime}}+1>0 \text { for every } \sigma, \sigma^{\prime} \in \mathrm{I}_{w} .
$$

We put

$$
\mathfrak{X}_{\Lambda}^{s e p}=\left\{x \in \operatorname{Hom}_{\text {cont }}\left(\Gamma_{\mathcal{K}}, \mathbf{C}_{p}^{\times}\right)|x|_{D_{p}} \text { is separable }\right\}
$$

and $\mathfrak{X}_{R}^{c l s, s e p}=i_{R, \Lambda}^{-1}\left(\mathfrak{X}_{\Lambda}^{s e p}\right) \cap \mathfrak{X}_{R}^{c l s}$. One can verify easily that $\mathfrak{X}_{R}^{\text {cls,sep }}$ is Zariski dense in $\mathfrak{X}_{R}^{c l s}$.

Let $x \in \mathfrak{X}_{R}^{c l s, s e p}$. Note that the Hecke character associated to $x$ (the complex avatar of $x)$ is unramified at all places above $p$ and is of infinity type $k \Sigma+\underline{a}(1-c)$. It follows that the corresponding automorphic representation $\pi_{x}$ is unramified at all places above $p$, and the $L$-parameter $\phi_{\pi_{x, \sigma}}: W_{\mathbf{C}}=\mathbf{C}^{\times} \rightarrow \mathrm{GL}_{3}(\mathbf{C})$ of the holomorphic discrete series $\pi_{x, \sigma}$ at an archimedean place $\sigma \in \Sigma$ is given by

$$
\phi_{\pi_{x, \sigma}}(z)=\operatorname{diag}\left((z / \bar{z})^{0},(z / \bar{z})^{-1-a_{\sigma}},(z / \bar{z})^{1-k-a_{\sigma}}\right) .
$$

On the other hand, consider the Galois representation $\rho_{x}=R_{p}\left(\pi_{x}\right)(1)$ associated to $\pi_{x}$. Let $w \in S_{p}^{\mathcal{K}}$ and let $D_{w}$ be the decomposition group of $w$ in $G_{\mathcal{K}}$. From the fact that the Galois representation $R_{p}\left(\pi_{x}\right)$ is constructed out of the $p$-adic étale cohomology groups of certain compact Picard modular surfaces with good reduction at places above $p$, we can deduce that $\rho_{x, w}:=\left.\rho_{x}\right|_{D_{w}}$ is an ordinary $p$-adic Galois representation since $\pi_{x}$ is a $p$-ordinary cuspidal automorphic representation Mai08, Thm.4.1.1(3)] ( $c f$. SU13, Lemma 7.2]). Suppose that $w \in \Sigma_{p}$. Then the local $p$-adic representation $\rho_{x, w}$ (resp. $\rho_{x, \bar{w}}$ ) is crystalline and has arithmetic HodgeTate weights

$$
\left(0,1+a_{\sigma}, k+a_{\sigma}-1\right)_{\sigma \in \mathrm{I}_{w}}\left(\operatorname{resp} .\left(1-a_{\sigma}-k,-a_{\sigma}-1,0\right)_{\sigma \in \mathrm{I}_{w}}\right)
$$

(cf. [BC04, Prop 3.3]). The Newton and Hodge polygons of $\rho_{x, w}$ meet at

$$
\left(d_{w}, 0\right),\left(2 d_{w}, \sum_{\sigma \in \mathrm{I}_{w}} 1+a_{\sigma}\right),\left(3 d_{w}, \sum_{\sigma \in \mathrm{I}_{w}} k+2 a_{\sigma}\right), d_{w}=\left[\mathcal{K}_{w}: \mathbf{Q}_{p}\right] .
$$

By [TU99, Lemma 7.2] (cf. [Mai08, Lemma 4.2.3]), the $R$-module $\mathscr{L}$ has a filtration $\{0\} \subset \mathscr{F}_{3, w} \subset \mathscr{F}_{1, w} \subset \mathscr{F}_{2, w}=\mathscr{L}$ of $R$-submodules, according to which $\boldsymbol{\rho}_{w}:=\left.\boldsymbol{\rho}\right|_{D_{w}}$ has the matrix representation

$$
\boldsymbol{\rho}_{w}=\left[\begin{array}{ccc}
\delta_{3, w}^{\prime} & * & * \\
& \delta_{1, w}^{\prime} & * \\
& & \delta_{2, w}^{\prime}
\end{array}\right] .
$$

For each $i=1,2,3$, the specialization $\delta_{i, w}^{\prime}(x)$ of $\delta_{i, w}^{\prime}$ at $x$ is a locally algebraic $p$-adic character of $D_{w}$ by a theorem of Serre [Ser68, Corollary, page III-50], and $\delta_{3, w}^{\prime}(x)$ has the maximal Hodge-Tate weights $k+a_{\sigma}-1$ for each $\sigma \in \mathrm{I}_{w}$. We remark that $\mathscr{F}_{3, w} \neq 0$ as $\mathcal{I} \neq R$ and $\mathscr{L} / \mathcal{I} \mathscr{L}$ has a quotient isomorphic to $\delta_{3} \otimes R / \mathcal{I}$, whereas 
the quotients $\mathscr{F}_{2, w} / \mathscr{F}_{1, w}$ and $\mathscr{F}_{1, w} / \mathscr{F}_{3, w}$ could be zero modules because $\mathscr{L}$ need not be a lattice. Let $\varepsilon_{w}$ be the $p$-adic character of $I_{w}$ induced by local class field theory $\varepsilon_{w}: I_{w} \rightarrow I_{w}^{a b} \stackrel{\sim}{\rightarrow} \mathcal{O}_{w}^{\times}$. Because the Hodge-Tate weights of $\left\{\delta_{i}(x)\right\}_{i=1,2,3}$ are distinct, by [Ser68, Thm. 2, page III-44] we find that

$$
\left.\delta_{3, w}^{\prime}(x)\right|_{I_{w}}=\prod_{\sigma \in I_{w}}\left(\sigma \varepsilon_{w}\right)^{k+a_{\sigma}-1}=\left.\delta_{3}(x)\right|_{I_{w}}
$$

for every $x \in \mathfrak{X}_{R}^{c l s, s e p}$ and $\left.\delta_{3, w}^{\prime}\right|_{I_{w}}=\left.\delta_{3}\right|_{I_{w}}$.

Lemma 8.7. Let $P \in \mathrm{ht}_{1}(\Lambda)$ such that $\mathcal{I}_{P} \subset P R_{P}$. Assume that

$$
\left.\delta_{i} \delta_{j}^{-1}\right|_{I_{w}} \not \equiv 1(\bmod P) \text { for all } i, j \text {. }
$$

Then the natural map $\left(\mathscr{F}_{3, w}\right)_{P} \rightarrow\left(\mathscr{L}_{3}\right)_{P}$ is surjective.

Proof. The assumption implies that $\left.\delta_{i, w}^{\prime}\right|_{I_{w}}=\left.\delta_{i}\right|_{I_{w}} \bmod P$ are distinct, so we can find $t$ in $R_{P}\left[I_{w}\right]$ such that $\left.\rho(t)\right|_{\mathscr{L}_{P} / P \mathscr{L}_{P}}$ is an idempotent, and according to the filtration $\{0\} \subset \mathscr{F}_{3, w} \subset \mathscr{F}_{1, w} \subset \mathscr{F}_{2, w}=\mathscr{L}$, we have

$$
\left.\boldsymbol{\rho}(t)\right|_{\mathscr{L}_{P} / P \mathscr{L}_{P}}=\left[\begin{array}{lll}
1 & * & * \\
& 0 & 0 \\
& & 0
\end{array}\right](\bmod P) .
$$

For $v \in\left(\mathscr{L}_{3}\right)_{P}$, we find that $\boldsymbol{\rho}(t) v \in\left(\mathscr{F}_{3, w}\right)_{P}$ and $\boldsymbol{\rho}(t) v \equiv v\left(\bmod P \mathscr{L}_{P}+\mathscr{D}_{P}\right)$, so the map $\left(\mathscr{F}_{3, w}\right)_{P} \rightarrow\left(\mathscr{L}_{3}\right)_{P} \otimes R_{P} / P$ is surjective. Therefore the map $\left(\mathscr{F}_{3, w}\right)_{P} \rightarrow$ $\left(\mathscr{L}_{3}\right)_{P}$ is surjective by Nakayama lemma.

Remark 8.8. We remark that the assumption in Lemma 8.7 is satisfied whenever $\operatorname{ord}_{P}\left(L_{p}^{S}(\Psi, \Sigma)\right)>0$. Indeed, if $\left.\delta_{i} \delta_{j}^{-1}\right|_{I_{w}} \equiv 1(\bmod P)$ for some $i, j$ and $w \in \Sigma_{p}$, then we can choose an integer $n_{0}$ large enough such that $\left(\Gamma_{\mathcal{K}}^{+}\right)^{p^{n_{0}}} \subset I_{w} \cap \Gamma_{\mathcal{K}}^{+}$for all $w \in \Sigma_{p}$. Thus

$$
\Psi^{1+c} \varepsilon^{-2}\left(\gamma_{+}^{p^{n_{0}}}\right) \equiv 1(\bmod P)
$$

and $P=\left(T_{+}-\left(\zeta_{0} \varepsilon\left(\gamma_{+}\right)-1\right)\right)$ for some $\zeta_{0} \in \boldsymbol{\mu}_{p^{n_{0}}}$. It follows that we can choose a point $y \in \mathfrak{X}_{\Lambda}$ such that $y(P)=0$ and $\Psi_{y}$ is a Hecke character of infinity type $(1+m) \Sigma+(1-m) \Sigma^{c}, m \geq 1$. The specialization of $L_{p}^{S}(\Psi, \Sigma)$ at $y$ is $L^{S}(m+1, \boldsymbol{\theta})$ for some Hilbert modular form $\boldsymbol{\theta}$ of parallel weight $(2 m+1)$, which is non-zero by Shi78, Prop. 4.16]. This in particular implies that $\operatorname{ord}_{P}\left(L_{p}^{S}(\Psi, \Sigma)\right)=0$.

Corollary 8.9. If $\operatorname{ord}_{P}\left(L_{p}^{S}(\Psi, \Sigma)\right)>0$, then there exists $t_{P} \in \Lambda-P$ such that

$$
\begin{aligned}
& \overline{\boldsymbol{c}}_{1}: t_{P}\left(\mathscr{I}_{1} / \mathscr{J}_{1}\right)^{*} \hookrightarrow \operatorname{Sel}_{\mathcal{K}}^{S}\left(\Psi^{-1-c} \varepsilon^{2}\right)^{c=-1} ; \\
& \overline{\boldsymbol{c}}_{2}: t_{P}\left(\mathscr{I}_{2} / \mathscr{J}_{2}\right)^{*} \hookrightarrow \operatorname{Sel}_{\mathcal{K}}^{S}\left(\Psi^{D}, \Sigma^{c}\right) .
\end{aligned}
$$

Proof. Injectivity of $\overline{\boldsymbol{c}}_{i}$ is proved in Lemma 8.6. By (8.4), for every $g \in G_{\mathcal{K}, S}$ we have

$$
a_{13}\left(g^{c}\right) a_{33}(g)+a_{23}\left(g^{c}\right) a_{23}(g)+a_{13}(g) a_{33}\left(g^{c}\right)=0
$$

and thus

$$
\delta_{3}(g) a_{13}\left(g^{c}\right)+a_{12}(g) a_{23}(g)+\delta_{3}\left(g^{c}\right) a_{13}(g) \equiv 0\left(\bmod \mathcal{I} R_{13}\right) .
$$

Let $f \in\left(\mathscr{I}_{1} / \mathscr{J}_{1}\right)^{*}$. Since $f\left(R_{12} R_{23}+\mathcal{I} R_{13}\right)=0$, we find that

$$
c_{1}(f)\left(g^{c}\right)=f\left(\delta_{3}^{-1}\left(g^{c}\right) a_{13}\left(g^{c}\right)\right)=-f\left(\delta_{3}^{-1}(g) a_{13}(g)\right)=-c_{1}(f)(g) .
$$

This shows that $\boldsymbol{c}_{1}(f)^{c}=-\boldsymbol{c}_{1}(f)$. 
For every $w \in \Sigma_{p}$, by Lemma 8.7 and Remark 8.8 there exists $u_{w} \in\left(R_{13}\right)_{P}$ and $v_{w} \in\left(R_{23}\right)_{P}$ such that $\left(u_{w}, v_{w}, 1\right)^{t} \in \mathscr{F}_{3, w}$. Choose $t_{P} \in \Lambda-P$ such that $u_{w}^{\prime}=t_{P} u_{w} \in R_{13}$ and $v_{w}^{\prime}=t_{P} v_{w} \in R_{23}$ for all $w \in \Sigma_{p}$. By the construction of $\mathscr{F}_{3, w}$, we have $\boldsymbol{\rho}(g)\left(u_{w}^{\prime}, v_{w}^{\prime}, t_{P}\right)^{t}=\delta_{3}(g)\left(u_{w}^{\prime}, v_{w}^{\prime}, t_{P}\right)^{t}$ for $g \in I_{w}$, from which we deduce the relations:

$$
\begin{aligned}
& \delta_{3}^{-1}(g) a_{12}(g) v_{w}^{\prime}+t_{P} \delta_{3}^{-1}(g) a_{13}(g)=\delta_{3}^{-1} \delta_{1}(g) u_{w}^{\prime}-u_{w}^{\prime}\left(\bmod \mathcal{I} R_{13}\right) ; \\
& \delta_{3}^{-1}(g) a_{21}(g) u_{w}^{\prime}+t_{P} \delta_{3}^{-1}(g) a_{23}(g)=\delta_{3}^{-1} \delta_{2}(g) v_{w}^{\prime}-v_{w}^{\prime}\left(\bmod \mathcal{I} R_{23}\right) .
\end{aligned}
$$

It follows that for every $f \in\left(\mathscr{I}_{i} / \mathscr{J}_{i}\right)^{*}, i \in\{1,2\}$, the class $\left[\boldsymbol{c}_{i}\left(t_{P} f\right)\right]$ is trivial in $H^{1}\left(I_{w}, \delta_{3}^{-1} \delta_{i} \otimes \Lambda^{*}\right)$. The morphisms $t_{P} \overline{\boldsymbol{c}}_{i}$ thus factor through the corresponding Selmer groups.

\subsection{Proof of the main theorem.}

8.5.1. After preparing some auxiliary results, we prove our main theorem (Theorem 8.14) in this subsection.

Lemma 8.10. The restriction map

$$
\text { res : } S e l_{\mathcal{F}}^{S}\left(\Psi_{+}^{-1} \tau_{\mathcal{K} / \mathcal{F}} \varepsilon^{2}\right) \stackrel{\sim}{\rightarrow} \operatorname{Sel}_{\mathcal{K}}^{S}\left(\Psi^{-1-c} \varepsilon^{2}\right)^{c=-1}
$$

is an isomorphism.

Proof. Since $\operatorname{Gal}(\mathcal{K} / \mathcal{F})=\langle c>$ has order two and $p>2$, the restriction map

$$
\text { res : } H^{1}\left(\mathcal{F}, \Psi_{+}^{-1} \tau_{\mathcal{K} / \mathcal{F}} \varepsilon^{2} \otimes \Lambda^{*}\right) \rightarrow H^{1}\left(\mathcal{K}, \Psi^{-1-c} \varepsilon^{2} \otimes \Lambda^{*}\right)^{c=-1} \quad\left(\left.\Psi_{+}\right|_{G_{\mathcal{K}}}=\Psi^{1+c}\right)
$$

is an isomorphism, and the lemma follows.

The following $S$-imprimitive version of the main conjecture for totally real fields is a key ingredient in our proof.

Theorem 8.11 (A. Wiles). For every $P \in \mathrm{ht}_{1}(\Lambda)$, we have

$$
\ell_{P}\left(X_{\mathcal{F}}^{S}\left(\Psi_{+}^{-1} \tau_{\mathcal{K} / \mathcal{F}} \varepsilon^{2}\right)\right) \leq \operatorname{ord}_{P}\left(L_{p}^{S}\left(-1, \Psi_{+} \tau_{\mathcal{K} / \mathcal{F}}\right)\right)
$$

If $\psi_{+} \neq \tau_{\mathcal{K} / \mathcal{F}} \omega_{\mathcal{F}}$ or Leopoldt's conjecture holds, then

$$
\ell_{P}\left(X_{\mathcal{F}}^{S}\left(\Psi_{+}^{-1} \tau_{\mathcal{K} / \mathcal{F}} \varepsilon^{2}\right)\right)=\operatorname{ord}_{P}\left(L_{p}^{S}\left(-1, \Psi_{+} \tau_{\mathcal{K} / \mathcal{F}}\right)\right) .
$$

Proof. It is clear that the character $\Psi_{+}^{-1} \tau_{\mathcal{K} / \mathcal{F}} \varepsilon^{2}$ factors through $\Lambda_{+}=\mathcal{O}_{\mathfrak{p}} \llbracket \Gamma_{\mathcal{K}}^{+} \rrbracket$. We identify $\Lambda_{+}$with $\mathcal{O}_{\mathfrak{p}} \llbracket \operatorname{Gal}\left(\mathcal{F}_{\infty} / \mathcal{F}\right) \rrbracket$ via the restriction map $\Gamma_{\mathcal{K}}^{+} \stackrel{\sim}{\rightarrow} \operatorname{Gal}\left(\mathcal{F}_{\infty} / \mathcal{F}\right)$. Write $\Psi_{1}$ for $\Psi_{+}^{-1} \tau_{\mathcal{K} / \mathcal{F}} \varepsilon^{2}$ when it is regarded as a $\Lambda_{+}$-valued character. Let $\mathcal{F}_{\infty}$ be the cyclotomic $\mathbf{Z}_{p}$-extension of $\mathcal{F}$. Since $\Lambda$ is flat over $\Lambda_{+}$, we have a $\Lambda$-module isomorphism $\operatorname{Sel}_{\mathcal{F}}^{S}\left(\Psi_{1}\right)^{*} \otimes_{\Lambda_{+}} \Lambda \simeq X_{\mathcal{F}}^{S}\left(\Psi_{+}^{-1} \tau_{\mathcal{K} / \mathcal{F}} \varepsilon^{2}\right)$. Therefore, the theorem is equivalent to

$$
\ell_{P}\left(\operatorname{Sel}_{\mathcal{F}}^{S}\left(\Psi_{1}\right)^{*}\right) \leq \operatorname{ord}_{P}\left(L_{p}^{S}\left(-1, \Psi_{+} \tau_{\mathcal{K} / \mathcal{F}}\right)\right)
$$

for all $P \in \mathrm{ht}_{1}\left(\Lambda_{+}\right)$. In view of the interpolation formula (6.14), we note that the $p$-adic $L$-function $L_{p}\left(-1, \Psi_{+} \tau_{\mathcal{K} / \mathcal{F}}\right)$ is nothing but $G_{\psi_{2}}\left(\varepsilon\left(\gamma_{+}^{-1}\right)\left(1+T_{+}\right)^{2}-1\right)$, where $G_{\psi_{2}}\left(T_{+}\right)$is the classical Deligne-Ribet $p$-adic $L$-function attached to the even character $\psi_{2}:=\psi_{+} \tau_{\mathcal{K} / \mathcal{F}} \omega_{\mathcal{F}}^{-1}$. From the main conjecture for totally real fields Wil90 and the exact sequence

$$
0 \rightarrow \operatorname{Sel}_{\mathcal{F}}\left(\Psi_{1}\right) \rightarrow \operatorname{Sel}_{\mathcal{F}}^{S}\left(\Psi_{1}\right) \stackrel{\gamma}{\rightarrow} \prod_{v \in S} H^{1}\left(\mathcal{F}_{v}, \Psi_{1} \otimes \Lambda_{+}^{*}\right),
$$


the equation (8.11) follows immediately except for the case $\psi_{+}=\tau_{\mathcal{K} / \mathcal{F}} \omega_{\mathcal{F}}$ and $P=P_{e}:=\left(T_{+}-\left(\varepsilon\left(\gamma_{+}^{\frac{1}{2}}\right)-1\right)\right)$, in which the Deligne-Ribet $p$-adic $L$-function $L_{p}\left(-1, \Psi_{+} \tau_{\mathcal{K} / \mathcal{F}}\right)$ conjecturally has a simple pole at $P_{e}$. Moreover, if $\psi_{+} \neq \tau_{\mathcal{K} / \mathcal{F}} \omega_{\mathcal{F}}$, coker $\gamma$ is trivial by [GV00, Prop. 2.1], and hence the inequality in (8.11) is indeed an equality. If $\psi_{+}=\tau_{\mathcal{K} / \mathcal{F}} \omega_{\mathcal{F}}$, using the global Poitou-Tate duality, one can show that there is an injective map

$$
\Lambda_{+} /\left(T_{+}-\left(\varepsilon\left(\gamma_{+}^{\frac{1}{2}}\right)-1\right)\right) \hookrightarrow(\operatorname{coker} \gamma)^{*}
$$

as $\Lambda_{+}$-modules, and this map is an isomorphism provided Leopoldt's conjecture holds for all $\mathcal{F}^{\prime}$ in $\mathcal{F} \subset \mathcal{F}^{\prime} \subset \mathcal{F}_{\infty}$. Thus, the inequality (8.11) also holds for $P=P_{e}$ by the main conjecture for totally real fields.

Proposition 8.12. For every $P \in \mathrm{ht}_{1}(\Lambda)$, we have

(1) $\ell_{P}\left(\operatorname{Sel}_{\mathcal{K}}(\Psi, \Sigma)\right)=\ell_{P}\left(\operatorname{Sel}_{\mathcal{K}}\left(\Psi^{D}, \Sigma^{c}\right)\right)$.

$$
\ell_{P}\left(\operatorname{Sel}_{\mathcal{K}}\left(\Psi^{D}, \Sigma^{c}\right)\right) \geq \ell_{P}\left(\operatorname{Sel}_{\mathcal{K}}^{S}\left(\Psi^{D}, \Sigma^{c}\right)\right)+\sum_{w \in S^{\mathcal{K}}, w \nmid \mathfrak{c}(\psi)} \ell_{P}\left(\left(1-\Psi\left(\operatorname{Frob}_{w}\right)\right)\right) .
$$

Proof. Part (1) is the functional equation of Selmer groups proved in Hsi10, Thm. 1.1], and part (2) is [Hsi10, Lemma 2.10].

Definition 8.13. A height one prime $P \in \mathrm{ht}_{1}(\Lambda)$ is said to be exceptional if

$$
\operatorname{ord}_{P}\left(L_{p}(\Psi, \Sigma)\right)>0 \text { and } \operatorname{ord}_{P}\left(L_{p}^{S}\left(-1, \Psi_{+} \tau_{\mathcal{K} / \mathcal{F}}\right)\right)>0 .
$$

Theorem 8.14. Let $P \in \mathrm{ht}_{1}(\Lambda)$ be a height one prime. Suppose that (8.3) holds. With the assumptions in Theorem $\mathbf{7 . 1 8}$,

(i) If $\operatorname{ord}_{P}\left(L_{p}^{S}(\Psi, \Sigma)\right)>0$, then $\ell_{P}\left(\operatorname{Sel}_{\mathcal{K}}^{S}\left(\Psi^{D}, \Sigma^{c}\right)\right)>0$.

(ii) If $P$ is not exceptional, then we have

$$
\ell_{P}\left(\operatorname{Sel}_{\mathcal{K}}^{S}\left(\Psi^{D}, \Sigma^{c}\right)\right) \geq \operatorname{ord}_{P}\left(L_{p}^{S}(\Psi, \Sigma)\right) .
$$

Therefore, it follows from Prop. 8.12 that

$$
\ell_{P}\left(\operatorname{Sel}_{\mathcal{K}}(\Psi, \Sigma)\right) \geq \operatorname{ord}_{P}\left(L_{p}(\Psi, \Sigma)\right) .
$$

Proof. The theorem is trivial if $\operatorname{ord}_{P}\left(L_{p}^{S}(\Psi, \Sigma)\right)=0$, so we may assume that $\operatorname{ord}_{P}\left(L_{p}^{S}(\Psi, \Sigma)\right)>0$ and hence $\mathcal{I}_{P} \subset P R_{P}$ in view of Cor. 7.20 Suppose further that $P$ is not exceptional. Then since the set $S$ consists of prime factors of the conductor of $\psi$ and primes split or ramified in $\mathcal{K}$, one can check that $\operatorname{ord}_{P}\left(L_{p}^{S}\left(-1, \Psi_{+} \tau_{\mathcal{K} / \mathcal{F}}\right)\right)=0$. It follows from Theorem 8.11 together with Cor. 8.9 and Lemma 8.10 that

$$
\begin{aligned}
\ell_{P}\left(X_{\mathcal{K}}^{S}\left(\Psi_{+}^{-1} \tau_{\mathcal{K} / \mathcal{F}} \varepsilon^{2}\right)^{c=-1}\right) & =\ell_{P}\left(X_{\mathcal{F}}^{S}\left(\Psi_{+}^{-1} \tau_{\mathcal{K} / \mathcal{F}} \varepsilon^{2}\right)\right)=0 ; \\
\left(\mathscr{I}_{1}\right)_{P} & =\left(\mathscr{J}_{1}\right)_{P} .
\end{aligned}
$$

By (8.6) and the relation $R_{12} R_{21} \subset \mathcal{I}$, we deduce that $\left(\mathscr{J}_{2}\right)_{P}=\left(\mathcal{I} \mathscr{I}_{2}\right)_{P}$.

Next we show that $\mathscr{I}_{2}=R_{23}$ is a faithful $R$-module. Let $\mathcal{C}$ denote the set of irreducible components of $R$. We decompose $F=\operatorname{Frac} R=\oplus_{g \in \mathcal{C}} F_{g}$ as a product of fields. For a fractional ideal $\mathscr{M}$ of $R$ in $F$, denote by $\mathscr{M}(g)$ the image of $\mathscr{M}$ in $F_{g}$ via the natural map $F \rightarrow F_{g}$. Then $\mathscr{M}(g)$ is a fractional ideal of $R(g)$ in $F_{g}$. We are going to show by contradiction that $\mathscr{I}_{2}(g) \neq 0$ for all $g \in \mathcal{C}$, 
which implies $\mathscr{I}_{2}$ is a faithful $R$-module. Suppose that $\mathscr{I}_{2}\left(g_{1}\right)=R_{23}\left(g_{1}\right)=0$ at some $g_{1} \in \mathcal{C}$. Then we have $R_{12}\left(g_{1}\right)_{P}=0$ by (8.4) and $\mathscr{I}_{1}\left(g_{1}\right)_{P}=\mathcal{I} \mathscr{I}_{1}\left(g_{1}\right)_{P}$ by (8.6). By the Nakayama lemma, $\mathscr{I}_{1}\left(g_{1}\right)_{P}=R_{13}\left(g_{1}\right)_{P}=0$, and hence $R_{13}\left(g_{1}\right)=$ 0 . It follows that the semi-simplification of the associated Galois representation $\boldsymbol{\rho}_{g_{1}}^{s s}: G_{\mathcal{K}, S} \rightarrow \mathrm{GL}_{3}\left(F_{g_{1}}\right)$ is isomorphic to a sum of three Galois characters. Let $x \in \mathfrak{X}_{R}^{c l s} \cap g_{1}$ be a classical point. The $L$-parameter of the associated cuspidal cohomological automorphic representation $\pi_{x}$ is a sum of three algebraic Hecke characters (Ser68). In addition, we can choose $x$ to have sufficiently regular weight such that $\pi_{x}$ is not CAP by a result of M. Harris [Har84, Thm. 2.5.6]. Thus, according to the classification of discrete $L$-packets for $U(2,1), \pi_{x}$ must be tempered endoscopic of type $(1,1,1)$, and the associated Galois representation $\rho_{x} \simeq \chi_{1} \oplus \chi_{2} \oplus$ $\chi_{3}, \chi_{i}^{-c}=\chi_{i}$, which contradicts the assumption (8.3) (cf. [Mai08, Lemma 5.0.7]).

The faithfulness of $\mathscr{I}_{2}$ implies that $\operatorname{Fitt}_{R}\left(\mathscr{I}_{2} / \mathcal{I} \mathscr{I}_{2}\right) \subset \mathcal{I}$. Put $X=X_{\mathcal{K}}^{S}\left(\Psi^{D}, \Sigma^{c}\right)$. By Cor. 8.9, we have

$$
\operatorname{Fitt}_{\Lambda}(X) R_{P}=\operatorname{Fitt}_{R_{P}}\left(X \otimes_{\Lambda} R_{P}\right) \subset \operatorname{Fitt}_{R_{P}}\left(\mathscr{I}_{2} / \mathcal{I} \mathscr{I}_{2}\right) \subset \mathcal{I}_{P}
$$

Because $R_{P}$ is finite and free over $\Lambda_{P}, \operatorname{Fitt}_{\Lambda}(X)_{P} \subset \mathcal{I}_{P} \cap \Lambda=\operatorname{Eis}(\Psi, S)_{P}$. Therefore by Cor. 7.20

$$
\ell_{P}\left(\operatorname{Sel}_{\mathcal{K}}^{S}\left(\Psi^{D}, \Sigma^{c}\right)\right)=\operatorname{ord}_{P}\left(\operatorname{Fitt}_{\Lambda}(X)\right) \geq \operatorname{ord}_{P}(\operatorname{Eis}(\Psi, S)) \geq \operatorname{ord}_{P}\left(L_{p}^{S}(\Psi, \Sigma)\right) .
$$

This completes the proof of part (ii).

Now we proceed to show part (i). If $\ell_{P}\left(\operatorname{Sel}_{\mathcal{K}}^{S}\left(\Psi^{D}, \Sigma^{c}\right)\right)=0$, then by the same argument as above, one can show that $\left(\mathscr{J}_{1}\right)_{P}=\mathcal{I}\left(\mathscr{I}_{1}\right)_{P}$ and $\mathscr{I}_{1}$ is a faithful $R$ module. Hence,

$$
\ell_{P}\left(X_{\mathcal{F}}^{S}\left(\Psi_{+} \tau_{\mathcal{K} / \mathcal{F}} \varepsilon^{2}\right)\right) \geq \operatorname{ord}_{P}\left(L_{p}^{S}(\Psi, \Sigma)\right)+\operatorname{ord}_{P}\left(L_{p}^{S}\left(-1, \Psi_{+} \tau_{\mathcal{K} / \mathcal{F}}\right)\right) .
$$

It follows from Theorem 8.11 that $\operatorname{ord}_{P}\left(L_{p}^{S}(\Psi, \Sigma)\right)=0$, which is a contradiction.

8.5.2. Let $\pi_{-}$be the restriction map $\pi_{-}: \Lambda \rightarrow \Lambda /\left(T_{+}\right) \stackrel{\sim}{\rightarrow} \Lambda_{-}=\mathcal{O}_{\mathfrak{p}} \llbracket \Gamma_{\mathcal{K}}^{-} \rrbracket$ and let $L_{p}^{-}(\Psi, \Sigma)=\pi_{-}\left(L_{p}(\Psi, \Sigma)\right) \in \Lambda_{-}$be the projection of $L_{p}(\Psi, \Sigma)$ to the anticyclotomic plane. We claim that there is no exceptional prime if the Iwasawa $\mu$-invariant $\mu_{\psi, \Sigma}^{-}$ of the anticyclotomic $p$-adic $L$-function $L_{p}^{-}(\Psi, \Sigma)$ vanishes. Indeed, $\mu_{\psi, \Sigma}^{-}=0$ if and only if $L_{p}(\Psi, \Sigma) \not \equiv 0\left(\bmod \mathfrak{m}_{\Lambda_{+}} \Lambda\right)$, where $\mathfrak{m}_{\Lambda_{+}}=\left(p, T_{+}\right)$is the maximal ideal of $\Lambda_{+}$. If $P$ is an exceptional prime, then $P$ is generated by a prime element in $\Lambda_{+}$. This in particular implies that $\operatorname{ord}_{P}\left(L_{p}(\Psi, \Sigma)\right)=0$, which is a contradiction. In virtue of Theorem 8.14, we have thus proved the corollary below:

Corollary 8.15. Assume that (8.3) and the following hypotheses hold:

(1) $p \nmid 3 \cdot h_{\mathcal{K}}^{-} \cdot D_{\mathcal{F}}$,

(2) $\psi$ is unramified at $\Sigma_{p}^{c}$, and $\psi \omega_{\mathcal{K}}^{-a}$ is unramified at $\Sigma_{p}$ for some integer a,

(3) $\mu_{p}(\psi)=0$, where $\mu_{p}(\psi)$ is the invariant defined in (7.11),

(4) either of the following two conditions holds:

(a) $\mu_{\psi, \Sigma}^{-}=0$;

(b) $\operatorname{ord}_{P}\left(L_{p}(\Psi, \Sigma)\right) \leq 1$ for every exceptional prime $P \in \mathrm{ht}_{1}(\Lambda)$.

Then we have

$$
\left(F_{\Lambda}(\Psi, \Sigma)\right) \subset\left(L_{p}(\Psi, \Sigma)\right)
$$


The following theorem is an immediate consequence of the above corollary combined with results on the vanishing of the $\mu$-invariants of anticyclotomic $p$-adic $L$ functions in Hsi13 and Bur13.

Theorem 8.16. Assume that the following hypotheses hold:

(1) $p \nmid 3 \cdot h_{\mathcal{K}}^{-} \cdot D_{\mathcal{F}} \cdot \sharp(\Delta)$,

(2) $\psi$ is unramified at $\Sigma_{p}^{c}$, and $\psi \omega_{\mathcal{K}}^{-a}$ is unramified at $\Sigma_{p}$ for some integer $a \not \equiv 2(\bmod p-1)$.

Then we have

$$
\left(F_{\Lambda}(\Psi, \Sigma)\right) \subset\left(L_{p}(\Psi, \Sigma)\right) .
$$

Proof. It suffices to verify the hypotheses in Cor. 8.15. Note that $p \nmid \sharp(\Delta)$ and (2) imply $\mu_{p}(\psi)=0$ and (8.3) respectively. To verify the assumption (4) in Cor. 8.15, we note that if either $\psi_{+} \neq \omega_{\mathcal{F}} \tau_{\mathcal{K} / \mathcal{F}}$ or $\psi_{+}=\omega_{\mathcal{F}} \tau_{\mathcal{K} / \mathcal{F}}$ with the root number $W_{\Sigma}(\psi) \equiv+1\left(\bmod \mathfrak{m}_{\Lambda}\right)$ (recall that $W_{\Sigma}(\psi) \in \Lambda^{\times}$is the root number in the functional equation of $\left.L_{\psi, \Sigma}\right)$, then $\mu_{p}(\psi)=0$ implies that $\mu_{\psi, \Sigma}^{-}=0$ by [Hsi13, Cor. 5.5, Thm. 6.12] (cf. [Hid10, Theorem I] if $\mathfrak{c}(\psi)^{-}=(1)$ ). On the other hand, if $\psi_{+}=\omega_{\mathcal{F}} \tau_{\mathcal{K} / \mathcal{F}}$ with $W_{\Sigma}(\psi) \equiv-1\left(\bmod \mathfrak{m}_{\Lambda}\right)$, then using the functional equation of Katz $p$-adic $L$-functions, we see that $L_{p}(\Psi, \Sigma)$ has a zero at $P_{e}=\left(T_{+}-\left(\varepsilon\left(\gamma_{+}^{\frac{1}{2}}\right)-1\right)\right)$. Write $L_{p}(\Psi, \Sigma)=P_{e} \cdot L_{p}^{\prime}(\Psi, \Sigma)$. By Bur13, Thm. A], the anticyclotomic projection

$$
\pi_{-}\left(L_{p}^{\prime}(\Psi, \Sigma)\right)=\left.\frac{\partial L_{p}(\Psi, \Sigma)}{\partial T_{+}}\right|_{T_{+}=\varepsilon\left(\gamma_{+}^{\frac{1}{2}}\right)-1} \in \Lambda_{-}
$$

has trivial $\mu$-invariant. This implies that no prime divisor of $L_{p}^{\prime}(\Psi, \Sigma)$ is contained in the ideal $\mathfrak{m}_{\Lambda_{+}} \Lambda$. Therefore, $P_{e}$ is the only possible exceptional height one prime and $\operatorname{ord}_{P_{e}}\left(L_{p}(\Psi, \Sigma)\right)=1$. This finishes the proof.

\subsection{Applications.}

8.6.1. Two cases of the main conjecture for $C M$ fields. Now we give two examples where the one-sided divisibility $L_{p}(\Psi, \Sigma) \mid F_{\Lambda}(\Psi, \Sigma)$ in Theorem8.16 actually implies the equality $\left(F_{\Lambda}(\Psi, \Sigma)\right)=\left(L_{p}(\Psi, \Sigma)\right)$, relying on the works of Hida and Rubin.

Theorem 8.17. Suppose that $p \nmid 3 \cdot h_{\mathcal{K}}^{-} \cdot D_{\mathcal{F}} \cdot \sharp(\Delta)$ and that

(H1) $\psi$ is anticyclotomic,

$(\mathrm{H} 2)$ the local character $\psi_{w}$ is unramified and non-trivial for every $w \in \Sigma_{p}$,

(H3) $\left.\psi\right|_{G_{\mathcal{K}\left(\sqrt{p^{*}}\right)}} \neq 1, p^{*}=(-1)^{\frac{p-1}{2}} p$.

Then

$$
\left(F_{\Lambda}(\Psi, \Sigma)\right)=\left(L_{p}(\Psi, \Sigma)\right) .
$$

Proof. If $d=[\mathcal{F}: \mathbf{Q}]=1$, this is the two-variable main conjecture for imaginary quadratic fields proved by Rubin. We assume $d \geq 2$. Notation is as in \$8.5.2. Let $F_{\Lambda}^{-}(\Psi, \Sigma)=\pi_{-}\left(F_{\Lambda}(\Psi, \Sigma)\right)$ and let $f_{\psi, \Sigma}^{a}=F_{\Lambda_{-}}\left(X_{\Sigma}^{(\psi)}\left(\mathcal{K}_{\infty}^{-}\right)\right) \in \Lambda_{-}$be the characteristic power series of $X_{\Sigma}^{(\psi)}\left(\mathcal{K}_{\infty}^{-}\right)$. By the case (i) in Remark 8.4, we have a $\Lambda_{-}$-module pseudo-isomorphism:

$$
\operatorname{Sel}_{\mathcal{K}}\left(\Psi, \Sigma, \Lambda_{-}\right) \sim \operatorname{Sel}_{\mathcal{K}}(\Psi, \Sigma, \Lambda)\left[T_{+}\right]
$$


Note that $\psi_{+} \neq \omega_{\mathcal{F}} \tau_{\mathcal{K} / \mathcal{F}}$ if $\psi$ is anticyclotomic and $p>3$. It follows from (8.1) and Theorem 8.16 that

$$
\left(f_{\psi, \Sigma}^{a}\right) \subset\left(F_{\Lambda}^{-}(\Psi, \Sigma)\right) \subset\left(L_{p}^{-}(\Psi, \Sigma)\right) .
$$

Hida [Hid06] proved that $\operatorname{Sel}_{\mathcal{K}}\left(\Psi, \Sigma, \Lambda_{-}\right)$is a cotorsion $\Lambda_{-}$-module (i.e. $f_{\psi, \Sigma}^{a} \neq 0$ ) and

$$
\left(f_{\psi, \Sigma}^{a}\right)=\left(L_{p}(\Psi, \Sigma)\right)\left(\bmod \left(T_{+}\right)\right)
$$

We deduce from (8.12) that

$$
\left(f_{\psi, \Sigma}^{a}\right)=\left(F_{\Lambda}^{-}(\Psi, \Sigma)\right)=\left(L_{p}^{-}(\Psi, \Sigma)\right)
$$

In particular $F_{\Lambda}^{-}(\Psi, \Sigma) \neq 0$; from Theorem 8.16 and $(8.13)$ it follows that

$$
\left(F_{\Lambda}(\Psi, \Sigma)\right)=\left(L_{p}(\Psi, \Sigma)\right) .
$$

Theorem 8.18. In addition to the hypotheses (1-2) in Theorem 8.16, we further suppose that

(R1) $\mathcal{K}=\mathcal{F} \mathcal{M}$, where $\mathcal{M}$ is an imaginary quadratic field in which $p$ splits,

(R2) $\Sigma$ is the p-ordinary CM-type of $\mathcal{K}$ obtained by extending the inclusion $\iota_{\infty}$ : $\mathcal{M} \hookrightarrow \mathbf{C}$,

(R3) $\mathcal{K}^{\prime}$ is abelian over $\mathcal{M}$ and $p \nmid\left[\mathcal{K}^{\prime}: \mathcal{M}\right]$.

Then

$$
\left(F_{\Lambda}(\Psi, \Sigma)\right)=\left(L_{p}(\Psi, \Sigma)\right) .
$$

Proof. Let $\Sigma_{0}$ be the CM-type of $\mathcal{M}$ induced by $\iota_{\infty}: \mathcal{M} \hookrightarrow$ C. Let $\Gamma_{0}$ be the kernel of the restriction map $\pi_{\mathcal{M}}: \Gamma_{\mathcal{K}} \rightarrow \Gamma_{\mathcal{M}}$ and let $I_{0}$ be the ideal of $\Lambda$ generated by $\left(\Psi\left(\Gamma_{0}\right)-1\right)$. Then $\Gamma_{0}$ is a free $\mathbf{Z}_{p}$-module of rank $d-1$ and $\pi_{\mathcal{M}}: \Lambda / I_{0} \Lambda \stackrel{\sim}{\rightarrow} \Lambda_{\mathcal{M}}$, where $\Lambda_{\mathcal{M}}=\mathcal{O}_{\mathfrak{p}} \llbracket \Gamma_{\mathcal{M}} \rrbracket$. As $p \nmid\left[\mathcal{K}^{\prime}: \mathcal{M}\right]$ and $\mathcal{K}^{\prime} / \mathcal{M}$ is abelian, we have an $\Lambda_{\mathcal{M}^{-}}$ module isomorphism

$$
\operatorname{Sel}_{\mathcal{K}}\left(\Psi, \Sigma, \Lambda_{\mathcal{M}}\right)=\bigoplus_{\psi_{\mathcal{M}}^{\prime}} \operatorname{Sel}_{\mathcal{M}}\left(\Psi_{\mathcal{M}}^{\prime}, \Sigma_{0}, \Lambda_{\mathcal{M}}\right)
$$

where $\psi_{\mathcal{M}}^{\prime}$ runs over characters of $\operatorname{Gal}\left(\mathcal{K}^{\prime} / \mathcal{M}\right)$ which extend $\psi$ and $\Psi_{\mathcal{M}}^{\prime}$ is the $\Lambda_{\mathcal{M}^{-}}$ adic character of $\psi_{\mathcal{M}}^{\prime}$. By the case (ii) in Remark 8.4 we have a $\Lambda_{\mathcal{M}}$-module pseudo-isomorphism $\operatorname{Sel}_{\mathcal{K}}\left(\Psi, \Sigma, \Lambda_{\mathcal{M}}\right) \sim \operatorname{Sel}_{\mathcal{K}}(\Psi, \Sigma)\left[I_{0}\right]$, and therefore

$$
\prod_{\psi_{\mathcal{M}}^{\prime}} \operatorname{char}_{\Lambda_{\mathcal{M}}} X_{\mathcal{M}}\left(\Psi_{\mathcal{M}}^{\prime}, \Sigma_{0}, \Lambda_{\mathcal{M}}\right) \subset \pi_{\mathcal{M}}\left(F_{\Lambda}(\Psi, \Sigma)\right)
$$

On the other hand, we have the following factorization of $p$-adic $L$-functions as ideals in $\Lambda_{\mathcal{M}}$

$$
\left(\pi_{\mathcal{M}}\left(L_{p}(\Psi, \Sigma)\right)\right)=\left(\prod_{\psi_{\mathcal{M}}^{\prime}} L_{p}\left(\Psi_{\mathcal{M}}^{\prime}, \Sigma_{0}\right)\right) .
$$

As in the proof of Theorem 8.17, the assertion follows from the two-variable main conjecture of imaginary quadratic fields and Theorem 8.16 
8.6.2. Main conjectures for elliptic curves with complex multiplication. Let $E$ be an elliptic curve defined over $\mathcal{F}$ with complex multiplication by the ring of integers of an imaginary quadratic field $\mathcal{M}$. Suppose $E$ has good ordinary reduction at all places of $\mathcal{F}$ above $p$, which implies that $p$ splits in $\mathcal{M}$. Write $p \mathcal{O}_{\mathcal{M}}=\mathfrak{p} \overline{\mathfrak{p}}$, where $\mathfrak{p}$ is the prime induced by $\iota_{p}: \mathcal{M} \hookrightarrow \mathbf{C}_{p}$. Let $\mathcal{K}=\mathcal{F} \mathcal{M}$ and let $\Sigma$ be the CM-type of $\mathcal{K}$ induced from $\iota_{\infty}: \mathcal{M} \hookrightarrow \mathbf{C}$. Let $\mathcal{K}^{\prime}=\mathcal{K}(E[\mathfrak{p}])$ and let $\psi:=\psi_{E}$ be the character obtained from the $\mathbf{Z}_{p}$-representation of $\Delta=\operatorname{Gal}\left(\mathcal{K}^{\prime} / \mathcal{K}\right)$ on $E[\mathfrak{p}]$. Let $\mathcal{K}$ be the unique $\mathbf{Z}_{p}^{2}$-extension of $\mathcal{K}$ in $\mathcal{K}\left(E\left[p^{\infty}\right]\right)$ and let $\Lambda_{E}:=\mathbf{Z}_{p} \llbracket \operatorname{Gal}(\mathcal{K} / \mathcal{K}) \rrbracket$ be the two-variable Iwasawa algebra over $\mathbf{Z}_{p}$. Note that $\mathcal{K}_{\infty}^{+} \subset \mathcal{K} \subset \mathcal{K}_{\infty}$. Define the $\mathfrak{p}$-Selmer group $\operatorname{Sel}_{\mathcal{K}}(E)_{\mathfrak{p}}$ attached to $E_{/ \mathcal{K}}$ by

$$
\operatorname{Sel}_{\mathcal{K}}(E)_{\mathfrak{p}}=\operatorname{ker}\left\{H^{1}\left(\mathcal{K}, E\left[\mathfrak{p}^{n}\right]\right) \rightarrow \prod_{w} H^{1}\left(\mathcal{K}_{w}, E\right)\right\} .
$$

Then $\operatorname{Sel}_{\mathcal{K}}(E)_{\mathfrak{p}}$ is a cofinitely generated discrete $\Lambda_{E}$-module, and by $\mathrm{PR} 84$, Ch. II, Thm. 18], we have a $\Lambda_{E}$-module isomorphism

$$
\operatorname{Sel}_{\mathcal{K}}(E)_{\mathfrak{p}} \stackrel{\sim}{\rightarrow} \operatorname{Hom}_{\mathbf{z}_{p}}\left(X_{\Sigma}^{(\psi)}(\mathcal{K}), E\left[\mathfrak{p}^{\infty}\right]\right) .
$$

Let $F_{\mathfrak{p}}(E) \in \Lambda_{E}$ denote the characteristic power series of $\operatorname{Sel}_{\mathcal{K}}(E)_{\mathfrak{p}}$. Let $\alpha_{E, \mathfrak{p}}$ be the $\mathbf{Z}_{p}$-valued character given by

$$
\alpha_{E, \mathfrak{p}}: \operatorname{Gal}(\mathcal{K} / \mathcal{K}) \hookrightarrow \operatorname{Gal}\left(\mathcal{K}\left(E\left[p^{\infty}\right]\right) / \mathcal{K}\right) \rightarrow \operatorname{Aut} E\left[\mathfrak{p}^{\infty}\right]=\mathbf{Z}_{p}^{\times}
$$

and let $\pi_{E, \mathfrak{p}}: \Lambda \rightarrow \Lambda_{E}$ be the morphism induced by the character $\tilde{\alpha}: \operatorname{Gal}\left(\mathcal{K}_{\infty} / \mathcal{K}\right) \rightarrow$ $\Lambda_{E}^{\times}, \tilde{\alpha}(g)=\left.\alpha_{E, \mathfrak{p}}\left(\left.g\right|_{\mathcal{K}}\right) g\right|_{\mathcal{K}}$. Let $\mathfrak{c}$ be the prime-to- $p$ conductor of $\psi$. It is well known that $\mathcal{K}^{\prime} \subset \mathcal{K}(\mathfrak{c p})$ if $p \nmid 6$. Let $\Lambda_{E, \mathcal{O}_{\mathfrak{p}}}:=\Lambda_{E} \otimes \mathbf{z}_{p} \mathcal{O}_{\mathfrak{p}}$. Define the two-variable $p$-adic $L$-function attached to $(E, \mathfrak{p})$ by

$$
L_{\mathfrak{p}}(E):=\pi_{E, \mathfrak{p}}\left(L_{p}(\Psi, \Sigma)\right) \in \Lambda_{E, \mathcal{O}_{\mathfrak{p}}},
$$

where $L(\Psi, \Sigma)$ is the $p$-adic $L$-function attached to the branch character $\psi: \Delta_{\mathfrak{c}} \rightarrow$ $\Delta \rightarrow \mu_{p-1} \hookrightarrow \mathbf{Z}_{p}^{\times}$.

Corollary 8.19. Suppose that $p \nmid 6 \cdot h_{\mathcal{K}}^{-} \cdot D_{\mathcal{F}}$ and that $E$ has good ordinary reduction at all places above $p$. Then we have the following inclusion between ideals in $\Lambda_{E, \mathcal{O}_{\mathfrak{p}}}$ :

$$
\left(F_{\mathfrak{p}}(E)\right) \subset\left(L_{\mathfrak{p}}(E)\right) .
$$

Proof. We apply Theorem 8.16 to the branch character $\psi=\psi_{E}$. Then $\psi_{+}=$ $\tau_{\mathcal{K} / \mathcal{F}} \omega_{\mathcal{F}}$, and (1-2) in Theorem 8.16 are satisfied under our assumptions. The corollary thus follows from Theorem 8.16 combined with the specialization principle in Remark 8.4 and (8.14).

We consider the cyclotomic main conjecture for $E_{/ \mathcal{F}}$. Let $\Lambda_{\mathcal{F}}=\mathbf{Z}_{p} \llbracket \operatorname{Gal}\left(\mathcal{F}_{\infty} / \mathcal{F}\right) \rrbracket$ and let $\pi_{+}: \Lambda_{E} \rightarrow \Lambda_{+} \simeq \Lambda_{\mathcal{F}}$ be the restriction map. Let $\Lambda_{\mathcal{F}, \mathcal{O}_{\mathfrak{p}}}:=\Lambda_{E} \otimes \mathbf{z}_{p} \mathcal{O}_{\mathfrak{p}}$ and let $L_{p}\left(E / \mathcal{F}_{\infty}\right):=\pi_{+}\left(L_{\mathfrak{p}}(E)\right) \in \Lambda_{\mathcal{F}, \mathcal{O}_{\mathfrak{p}}}$ be the cyclotomic $p$-adic $L$-function for $E$. Let $\Omega_{E}:=\prod_{\sigma \in \mathrm{I}_{\mathcal{F}}} \Omega_{E}^{(\sigma)}$ be the period of $E$, where $E^{\sigma}$ is the $\sigma$-conjugate of $E$ for $\sigma \in \mathrm{I}_{\mathcal{F}}$ and $\Omega_{E}^{(\sigma)}=\int_{E^{\sigma}(\mathbf{R})} \boldsymbol{\omega}_{E^{\sigma}}$ is the period of a Néron differential $\boldsymbol{\omega}_{E}^{(\sigma)}$ of $E^{\sigma}$ over $\mathcal{O}_{\mathcal{F}^{\sigma},(p)}$. We remark that the complex CM period $\Omega_{\infty}^{1 \cdot \Sigma}$ in the interpolation formula of $L_{p}(\Psi, \Sigma)$ (see $\sqrt{6.5}$ ) in fact equals $c \cdot \Omega_{E}$ for some $c \in \overline{\mathbf{Z}}_{(p)}^{\times}$. On the other hand, Shapiro's lemma shows that $\operatorname{Sel}_{\mathcal{F}_{\infty}}(E) \stackrel{\sim}{\rightarrow} \operatorname{Sel}_{\mathcal{K}_{\infty}^{+}}(E)_{\mathfrak{p}}$ as $\Lambda_{\mathcal{F}}$-modules for odd $p$. 
Specializing everything in Cor. 8.19 to the cyclotomic line and using the descent of Selmer groups for CM elliptic curves [PR84, Ch. II, Lemma 9 and Prop. 12], we obtain the following theorem.

Theorem 8.20. With the assumptions in Cor. 8.19, we have the inclusion between ideals in $\Lambda_{\mathcal{F}, \mathcal{O}_{\mathrm{p}}}$

$$
\left(\operatorname{char}_{\Lambda_{\mathcal{F}}} \operatorname{Sel}_{\mathcal{F}_{\infty}}(E)\right) \subset\left(L_{p}\left(E / \mathcal{F}_{\infty}\right)\right) .
$$

\section{ACKNOWLEDGMENTS}

The author learned the fundamental ideas in this article from a preprint Urb06] of Eric Urban and the oral communication by him when the author was his Ph.D. student at Columbia University. This work was impossible without his insight and guidance.

The influence of Hida's ideas and works in this article is evident. The author would like to thank him for sharing his mathematical ideas by writing so many excellent books and also for answering questions with patience during the preparation of this article. He also thanks Prof. Coates and Prof. Tilouine for their encouragement and helpful suggestions and A. Burungale for sending his preprint to the author, which leads to the removal of the global root assumption in the previous version of this manuscript.

Part of the material in $\$ 8$ grew from the courses in the summer school for Iwasawa theory held in McMaster University during August 19-23, 2007. The author would like to thank the organizers Prof. Kolster and Prof. Sharifi for such a wonderful summer school.

Finally, the author is very grateful to the referees for a careful reading of the manuscript and valuable suggestions for the improvement of the exposition.

\section{REFERENCES}

[BC04] Joël Bellaïche and Gaëtan Chenevier, Formes non tempérées pour $\mathrm{U}(3)$ et conjectures de Bloch-Kato (French, with English and French summaries), Ann. Sci. École Norm. Sup. (4) 37 (2004), no. 4, 611-662, DOI 10.1016/j.ansens.2004.05.001. MR2097894 (2005m:11096)

[BC09] Joël Bellaïche and Gaëtan Chenevier, Families of Galois representations and Selmer groups, Astérisque 324 (2009), xii+314.

[BR93] Don Blasius and Jonathan D. Rogawski, Motives for Hilbert modular forms, Invent. Math. 114 (1993), no. 1, 55-87, DOI 10.1007/BF01232663. MR.1235020 (94i:11033)

[Bur13] A. Burungale, On the $\mu$-invariant of cyclotomic derivative of Katz p-adic L-functions, J. Inst. Math. Jussieu, posted on 2014, DOI 10.1017/S1474748013000388.

[Cas80] W. Casselman, The unramified principal series of $\mathfrak{p}$-adic groups. I. The spherical function, Compositio Math. 40 (1980), no. 3, 387-406. MR571057 (83a:22018)

[CG83] John Coates and Catherine Goldstein, Some remarks on the main conjecture for elliptic curves with complex multiplication, Amer. J. Math. 105 (1983), no. 2, 337-366, DOI 10.2307/2374263. MR701564 (85d:11100)

[Cha85] Ching-Li Chai, Compactification of Siegel moduli schemes, London Mathematical Society Lecture Note Series, vol. 107, Cambridge University Press, Cambridge, 1985. MR 853543 (88b:32074)

[Cha08] Ching-Li Chai, Methods for p-adic monodromy, J. Inst. Math. Jussieu 7 (2008), no. 2, 247-268, DOI 10.1017/S1474748007000199. MR2400722 (2009f:14045)

[DR80] Pierre Deligne and Kenneth A. Ribet, Values of abelian L-functions at negative integers over totally real fields, Invent. Math. 59 (1980), no. 3, 227-286, DOI 10.1007/BF01453237. MR.579702 (81m:12019) 
[FC90] G. Faltings and C.-L. Chai, Degeneration of abelian varieties, Ergebnisse der Mathematik und ihrer Grenzgebiete (3), vol. 22, Springer-Verlag, Berlin, 1990. With an appendix by David Mumford.

[Fin06] Tobias Finis, Divisibility of anticyclotomic L-functions and theta functions with complex multiplication, Ann. of Math. (2) 163 (2006), no. 3, 767-807, DOI 10.4007/annals.2006.163.767. MR 2215134(2007m:11151)

[Fuj89] K. Fujiwara, Arithmetic compactifications of Shimura varieties (I), Master's Thesis, University of Tokyo, 1989.

[GPSR87] Stephen Gelbart, Ilya Piatetski-Shapiro, and Stephen Rallis, Explicit constructions of automorphic L-functions, Lecture Notes in Mathematics, vol. 1254, Springer-Verlag, Berlin, 1987. MR892097 (89k:11038)

[Gre94] Ralph Greenberg, Iwasawa theory and p-adic deformations of motives, Motives (Seattle, WA, 1991), Proc. Sympos. Pure Math., vol. 55, Amer. Math. Soc., Providence, RI, 1994, pp. 193-223. MR.1265554 (95i:11053)

[Gre99] R. Greenberg, Iwasawa theory for elliptic curves, Lecture Notes in Math., vol. 1716, Springer, Berlin, 1999. MR,1754686 (2002a:11056)

[GV00] Ralph Greenberg and Vinayak Vatsal, On the Iwasawa invariants of elliptic curves, Invent. Math. 142 (2000), no. 1, 17-63, DOI 10.1007/s002220000080. MR1784796 (2001g:11169)

[Har84] Michael Harris, Eisenstein series on Shimura varieties, Ann. of Math. (2) 119 (1984), no. 1, 59-94, DOI 10.2307/2006963. MR736560 (85j:11052)

[Hid93] Haruzo Hida, Elementary theory of L-functions and Eisenstein series, London Mathematical Society Student Texts, vol. 26, Cambridge University Press, Cambridge, 1993. MR.1216135 (94j:11044)

[Hid95] Haruzo Hida, Control theorems of p-nearly ordinary cohomology groups for SL $(n)$ (English, with English and French summaries), Bull. Soc. Math. France 123 (1995), no. 3, 425-475. MR.1373742 (97c:11053)

[Hid98] Haruzo Hida, Automorphic induction and Leopoldt type conjectures for GL(n), Asian J. Math. 2 (1998), no. 4, 667-710. Mikio Sato: a great Japanese mathematician of the twentieth century. MR 1734126 (2000k:11064)

[Hid02] Haruzo Hida, Control theorems of coherent sheaves on Shimura varieties of PEL type, J. Inst. Math. Jussieu 1 (2002), no. 1, 1-76, DOI 10.1017/S1474748002000014. MR.1954939 (2003m:11086)

[Hid04a] H. Hida, Non-vanishing modulo $p$ of Hecke L-values, Geometric aspects of dwork theory. vol. I, II, 2004, pp. 735-784.

[Hid04b] H. Hida, p-adic automorphic forms on Shimura varieties, Springer Monographs in Mathematics, Springer-Verlag, New York, 2004.

[Hid06] Haruzo Hida, Anticyclotomic main conjectures, Doc. Math. Extra Vol. (2006), 465532 (electronic). MR 2290595 (2007m:11152)

[Hid07] H. Hida, Non-vanishing modulo $p$ of Hecke L-values and application, L-functions and Galois representations, 2007, pp. 207-269.

[Hid09a] Haruzo Hida, Irreducibility of the Igusa tower, Acta Math. Sin. (Engl. Ser.) 25 (2009), no. 1, 1-20, DOI 10.1007/s10114-008-6490-z. MR2465518 (2010f:11098)

[Hid09b] H. Hida, Quadratic exercises in Iwasawa theory, Int. Math. Res. Not. IMRN 5 (2009), 912-952.

[Hid10] Haruzo Hida, The Iwasawa $\mu$-invariant of p-adic Hecke L-functions, Ann. of Math. (2) 172 (2010), no. 1, 41-137, DOI 10.4007/annals.2010.172.41. MR2680417(2012d:11215)

[HLS06] Michael Harris, Jian-Shu Li, and Christopher M. Skinner, p-adic L-functions for unitary Shimura varieties. I. Construction of the Eisenstein measure, Doc. Math. Extra Vol. (2006), 393-464 (electronic). MR2290594 (2008d:11042)

[Hsi10] Ming-Lun Hsieh, The algebraic functional equation of Selmer groups for CM fields, J. Number Theory 130 (2010), no. 9, 1914-1924, DOI 10.1016/j.jnt.2010.02.003. MR2653204 (2011f:11143)

[Hsi11] Ming-Lun Hsieh, Ordinary p-adic Eisenstein series and p-adic L-functions for unitary groups (English, with English and French summaries), Ann. Inst. Fourier (Grenoble) 61 (2011), no. 3, 987-1059, DOI 10.5802/aif.2635. MR.2918724 
[Hsi12] Ming-Lun Hsieh, On the non-vanishing of Hecke L-values modulo p, Amer. J. Math. 134 (2012), no. 6, 1503-1539, DOI 10.1353/ajm.2012.0049. MR.2999287

[Hsi13] M.-L. Hsieh, On the $\mu$-invariant of anticyclotomic p-adic L-functions for CM fields, J. Reine Angew. Math., posted on 2013, DOI 10.1515/crelle-2012-0056. ArXiv:1112.1574.

[HT93] H. Hida and J. Tilouine, Anti-cyclotomic Katz p-adic L-functions and congruence modules, Ann. Sci. École Norm. Sup. (4) 26 (1993), no. 2, 189-259. MR 1209708 (93m:11044)

[HT94] H. Hida and J. Tilouine, On the anticyclotomic main conjecture for CM fields, Invent. Math. 117 (1994), no. 1, 89-147, DOI 10.1007/BF01232236. MR1269427(95d:11149)

[Jan87] Jens Carsten Jantzen, Representations of algebraic groups, Pure and Applied Mathematics, vol. 131, Academic Press Inc., Boston, MA, 1987. MR899071 (89c:20001)

[Kat78] Nicholas M. Katz, p-adic L-functions for CM fields, Invent. Math. 49 (1978), no. 3, 199-297, DOI 10.1007/BF01390187. MR.513095 (80h:10039)

[Kat81] N. Katz, Serre-Tate local moduli, Algebraic surfaces (orsay, 1976-78), 1981, pp. 138-202.

[Kot92] Robert E. Kottwitz, Points on some Shimura varieties over finite fields, J. Amer. Math. Soc. 5 (1992), no. 2, 373-444, DOI 10.2307/2152772. MR1124982 (93a:11053)

[Lan76] Robert P. Langlands, On the functional equations satisfied by Eisenstein series, Lecture Notes in Mathematics, Vol. 544, Springer-Verlag, Berlin, 1976. MR0579181(58 \#28319)

[Lan08] K.-W. Lan, Arithmetic compactification of PEL-type Shimura varieties, Ph.D. Thesis, Harvard University, 2008.

[Lan12] Kai-Wen Lan, Comparison between analytic and algebraic constructions of toroidal compactifications of PEL-type Shimura varieties, J. Reine Angew. Math. 664 (2012), 163-228. MR2980135

[Li92] Jian-Shu Li, Nonvanishing theorems for the cohomology of certain arithmetic quotients, J. Reine Angew. Math. 428 (1992), 177-217, DOI 10.1515/crll.1992.428.177. MR:1166512 (93e:11067)

[Mai04] F. Mainardi, Sur la conjecture principale de iwasawa pour les corps CM, Ph.D. Thesis, Universite Paris XIII, 2004.

[Mai08] Fabio Mainardi, On the main conjecture for CM fields, Amer. J. Math. 130 (2008), no. 2, 499-538, DOI 10.1353/ajm.2008.0019. MR2405166 (2010d:11129)

[Mau04] David Mauger, Algèbres de Hecke quasi-ordinaires universelles (French, with English and French summaries), Ann. Sci. École Norm. Sup. (4) 37 (2004), no. 2, 171-222, DOI 10.1016/j.ansens.2004.01.001. MR.2061780(2005i:11072)

[MS00] Atsushi Murase and Takashi Sugano, Local theory of primitive theta functions, Compositio Math. 123 (2000), no. 3, 273-302, DOI 10.1023/A:1002051017269. MR.1795292 (2001j:11021)

[MS02] Atsushi Murase and Takashi Sugano, Fourier-Jacobi expansion of Eisenstein series on unitary groups of degree three, J. Math. Sci. Univ. Tokyo 9 (2002), no. 2, 347-404. MR1904935 (2003f:11065)

[MS07] Atsushi Murase and Takashi Sugano, On the Fourier-Jacobi expansion of the unitary Kudla lift, Compos. Math. 143 (2007), no. 1, 1-46.

[Mum70] David Mumford, Abelian varieties, Tata Institute of Fundamental Research Studies in Mathematics, No. 5, Published for the Tata Institute of Fundamental Research, Bombay, 1970. MR0282985 (44 \#219)

[MW84] B. Mazur and A. Wiles, Class fields of abelian extensions of Q, Invent. Math. 76 (1984), no. 2, 179-330, DOI 10.1007/BF01388599. MR742853 (85m:11069)

[Nek06] Jan Nekovář, Selmer complexes (English, with English and French summaries), Astérisque 310 (2006), viii+559. MR.2333680 (2009c:11176)

[PR84] B. Perrin-Riou, Arithmétique des courbes elliptiques et théorie d'Iwasawa, Mém. Soc. Math. France (N.S.) 17 (1984), 130.

[Rib76] Kenneth A. Ribet, A modular construction of unramified p-extensions of $\left(\mu_{p}\right)$, Invent.

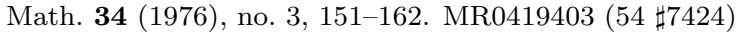

[Rog92] Jonathan D. Rogawski, Analytic expression for the number of points mod $p$, The zeta functions of Picard modular surfaces, Univ. Montréal, Montreal, QC, 1992, pp. 65-109. MR.1155227 (93i:11140) 
[Roh82] David E. Rohrlich, Root numbers of Hecke L-functions of CM fields, Amer. J. Math. 104 (1982), no. 3, 517-543, DOI 10.2307/2374152. MR658544(83j:12011)

[Rub91] Karl Rubin, The "main conjectures" of Iwasawa theory for imaginary quadratic fields, Invent. Math. 103 (1991), no. 1, 25-68, DOI 10.1007/BF01239508. MR1079839 (92f:11151)

[Ser68] Jean-Pierre Serre, Abelian l-adic representations and elliptic curves, McGill University lecture notes written with the collaboration of Willem Kuyk and John Labute, W. A. Benjamin, Inc., New York-Amsterdam, 1968. MR0263823 (41 \#8422)

[SGA64] Schémas en groupes. II: Groupes de type multiplicatif, et structure des schémas en groupes généraux, Séminaire de Géométrie Algébrique du Bois Marie 1962/64 (SGA 3), Vol. 152, Springer-Verlag, Berlin, 1962/1964. Dirigé par M. Demazure et A. Grothendieck. Lecture Notes in Mathematics.

[SGA71] Revêtements étales et groupe fondamental (French), Springer-Verlag, Berlin, 1971. Séminaire de Géométrie Algébrique du Bois Marie 1960-1961 (SGA 1); Dirigé par Alexandre Grothendieck. Augmenté de deux exposés de M. Raynaud; Lecture Notes in Mathematics, Vol. 224. MR0354651 (50 \#7129)

[Shi78] Goro Shimura, The special values of the zeta functions associated with Hilbert modular forms, Duke Math. J. 45 (1978), no. 3, 637-679. MR507462 (80a:10043)

[Shi79] T. Shintani, On automorphic forms on unitary groups of oreder 3, 1979. unpublished.

[Shi83] Goro Shimura, On Eisenstein series, Duke Math. J. 50 (1983), no. 2, 417-476. MR705034 (84k:10019)

[Shi97] G. Shimura, Euler products and Eisenstein series, CBMS Regional Conference Series in Mathematics, vol. 93, Published for the Conference Board of the Mathematical Sciences, Washington, DC, 1997.

[Shi98] Goro Shimura, Abelian varieties with complex multiplication and modular functions, Princeton Mathematical Series, vol. 46, Princeton University Press, Princeton, NJ, 1998. MR1492449 (99e:11076)

[SU13] C. Skinner and E. Urban, The Iwasawa Main Conjectures for $G L_{2}$, Inventiones Mathematicae, posted on 2013, DOI 10.1007/s00222-013-0448-1.

[TU99] J. Tilouine and E. Urban, Several-variable p-adic families of Siegel-Hilbert cusp eigensystems and their Galois representations (English, with English and French summaries), Ann. Sci. École Norm. Sup. (4) 32 (1999), no. 4, 499-574, DOI 10.1016/S00129593(99)80021-4. MR.1693583(2000j:11064)

[Urb99] E. Urban, On residually reducible representations on local rings, J. Algebra 212 (1999), no. 2, 738-742, DOI 10.1006/jabr.1998.7635. MR1676863(2000a:16020)

[Urb01] Eric Urban, Selmer groups and the Eisenstein-Klingen ideal, Duke Math. J. 106 (2001), no. 3, 485-525, DOI 10.1215/S0012-7094-01-10633-9. MR1813234(2002b:11073)

[Urb06] Eric Urban, Groupes de Selmer er fonctions L p-adiques pour les représentations modulaires adjointes, 2006. Preprint.

[Wed99] Torsten Wedhorn, Ordinariness in good reductions of Shimura varieties of PELtype (English, with English and French summaries), Ann. Sci. École Norm. Sup. (4) 32 (1999), no. 5, 575-618, DOI 10.1016/S0012-9593(01)80001-X. MR1710754 (2000g:11054)

[Wil90] A. Wiles, The Iwasawa conjecture for totally real fields, Ann. of Math. (2) 131 (1990), no. 3, 493-540, DOI 10.2307/1971468. MR1053488(91i:11163)

[Yag82] Rodney I. Yager, On two variable p-adic L-functions, Ann. of Math. (2) 115 (1982), no. 2, 411-449, DOI 10.2307/1971398. MR647813 (84b:14020)

[Yan97] Tonghai Yang, Theta liftings and Hecke L-functions, J. Reine Angew. Math. 485 (1997), 25-53, DOI 10.1515/crll.1997.485.25. MR.1442188(98e:11056)

Department of Mathematics, National Taiwan University, No. 1, Sec. 4, Roosevelt RoAD, TAIPEI 10617, TAIWAN

E-mail address: mlhsieh@math.ntu.edu.tw 